\title{
Enantioselective Syntheses of 1,4-Pentadien-3-yl Carbinols via Brønsted Acid Catalysis
}

Shang Gao and Ming Chen*

Department of Chemistry and Biochemistry

Auburn University

E-mail: mzc0102@auburn.edu

Supporting Information: Experimental Procedures, Tabulated Spectroscopic Data, ${ }^{1} \mathrm{H}$ and

${ }^{13} \mathrm{C}$ Spectra of New Compounds

SI-1 
General Experimental Details. All reaction solvents were purified before use. Tetrahydrofuran, dichloromethane, diethyl ether and toluene were purified by passing through a solvent column composed of activated A-1 alumina. Unless indicated otherwise, all reactions were conducted under an atmosphere of argon using flame-dried or oven-dried $\left(120^{\circ} \mathrm{C}\right)$ glassware. The term "concentrated under reduced pressure" refers to the removal of solvents and other volatile materials using a rotary evaporator with the water bath temperature below $30{ }^{\circ} \mathrm{C}$, followed by removal of residual solvent at high vacuum $(<0.2$ mbar $)$.

Proton nuclear magnetic resonance $\left({ }^{1} \mathrm{H}\right.$ NMR) spectra were acquired on commercial instruments (400 and $600 \mathrm{MHz}$ ) at Auburn University NMR facility. Carbon-13 nuclear magnetic resonance $\left({ }^{13} \mathrm{C}\right.$ NMR) spectra were acquired at 101 and $151 \mathrm{MHz}$. The proton signal for residual non-deuterated solvent $\left(\delta 7.26\right.$ for $\left.\mathrm{CHCl}_{3}\right)$ was used as an internal reference for ${ }^{1} \mathrm{H}$ NMR spectra. For ${ }^{13} \mathrm{C}$ NMR spectra, chemical shifts are reported relative to the $\delta 77.36$ resonance of $\mathrm{CHCl}_{3}$. Coupling constants are reported in $\mathrm{Hz}$. Optical rotations were measured on a Perkin Elmer 241 Automatic Polarimeter. High-resolution mass spectra were recorded on a commercial high-resolution mass spectrometer via the Micro Mass/Analytical Facility operated by the College of Chemistry and Biochemistry, Auburn University.

Analytical thin layer chromatography (TLC) was performed on Kieselgel 60 F254 glass plates precoated with a $0.25 \mathrm{~mm}$ thickness of silica gel. The TLC plates were visualized with UV light and/or by staining with Hanessian solution (ceric sulfate and ammonium molybdate in aqueous sulfuric acid) or $\mathrm{KMnO}_{4}$. Column chromatography was generally performed using Kieselgel 60 (230-400 mesh) silica gel, typically using a 50-100:1 weight ratio of silica gel to crude product. 
Syntheses of allylboronate reagents 1a, 1c, 1d:

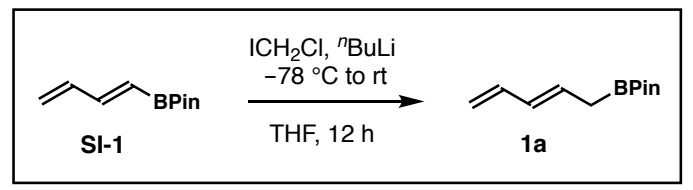

(E)-4,4,5,5-Tetramethyl-2-(penta-2,4-dien-1-yl)-1,3,2-dioxaborolane (1a) To a solution of boronate $\mathbf{S I - 1}{ }^{1}$ (2.0 mmol, 1.0 equiv) in THF $(10 \mathrm{~mL})$ was added $\mathrm{ICH}_{2} \mathrm{Cl}(3.0$ mmol, 1.5 equiv) under argon. The resulting colorless solution was placed in an acetone-dry ice bath and cooled to $-78{ }^{\circ} \mathrm{C}$. After stirring at $-78{ }^{\circ} \mathrm{C}$ for $10 \mathrm{~min}, n$-BuLi (2.5 $\mathrm{M}$ in THF, $0.88 \mathrm{~mL}, 2.2 \mathrm{mmol}, 1.1$ equiv) was added slowly to the reaction. After stirring at $-78{ }^{\circ} \mathrm{C}$ for $20 \mathrm{~min}$, the cooling bath was removed. The reaction mixture was allowed to warm to ambient temperature and stirred for $12 \mathrm{~h}$. Then the resulting mixture was cooled to $0{ }^{\circ} \mathrm{C}$ with an ice bath; diethyl ether $(20 \mathrm{~mL})$ and water $(10 \mathrm{~mL})$ were slowly added. The organic layer was separated and the aqueous layer was extracted with ethyl acetate $(3 \times 5 \mathrm{~mL})$. The combined organic extracts were dried over anhydrous sodium sulfate, filtered, and concentrated under reduced pressure. Purification of the crude product was performed by flash column chromatography (gradient elution with hexane and diethyl ether, 100:1 to 20:1) to give allylboronate 1 a as colorless oil in $c a$. $72 \%$ yield (318 mg, contain $\sim 10 \%$ SI-1). ${ }^{1} \mathrm{H}$ NMR (400 MHz, $\left.\mathrm{CDCl}_{3}\right) \delta 6.31$ (ddd, $J=17.0$, $10.3,10.3 \mathrm{~Hz}, 1 \mathrm{H}), 6.05$ (dd, $J=14.8,10.6 \mathrm{~Hz}, 1 \mathrm{H}), 5.76(\mathrm{dt}, J=15.2,7.7 \mathrm{~Hz}, 1 \mathrm{H}), 5.03$ $(\mathrm{d}, J=17.0 \mathrm{~Hz}, 1 \mathrm{H}), 4.90$ (d, $J=10.1 \mathrm{~Hz}, 1 \mathrm{H}), 1.75$ (d, $J=7.5 \mathrm{~Hz}, 2 \mathrm{H}), 1.25$ (s, 12H). ${ }^{13} \mathrm{C}$ NMR $\left(151 \mathrm{MHz}, \mathrm{CDCl}_{3}\right) \delta 137.8,131.9,130.9,114.2,83.7,25.1$. Compound 1a was also synthesized in a much lower yield by adopting the procedure reported by Schlosser. ${ }^{2}$

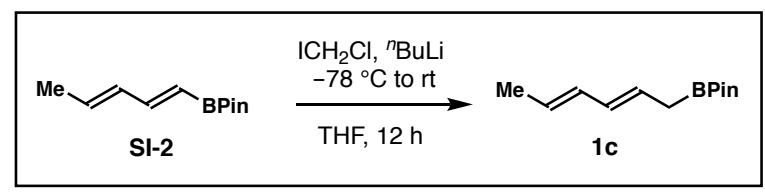

2-((2E,4E)-Hexa-2,4-dien-1-yl)-4,4,5,5-tetramethyl-1,3,2-dioxaborolane (1c) Prepared from SI-2 ${ }^{1}$ according to the procedure for the synthesis of $\mathbf{1 a}$. The crude mixture was purified by flash column chromatography (gradient elution with hexane and diethyl ether, 100:1 to 20:1) to give compound 1c as colorless oil in $c a$. $80 \%$ yield (374 $\mathrm{mg}$, contain $\sim 10 \%$ SI-2). ${ }^{1} \mathrm{H}$ NMR (600 MHz, $\left.\mathrm{CDCl}_{3}\right) \delta 5.96-6.04(\mathrm{~m}, 2 \mathrm{H}), 5.60$ (dt, $J=14.4,7.3$ $\mathrm{Hz}, 1 \mathrm{H}), 5.52(\mathrm{dq}, J=13.2,6.5 \mathrm{~Hz}, 1 \mathrm{H}), 1.70-1.72(\mathrm{~m}, 5 \mathrm{H}), 1.24(\mathrm{~s}, 12 \mathrm{H}) .{ }^{13} \mathrm{C} \mathrm{NMR}$ $\left(151 \mathrm{MHz}, \mathrm{CDCl}_{3}\right) \delta 132.1,131.2,127.2,126.2,83.5,25.0,18.4$. The spectroscopic data are consistent with literature data. ${ }^{2}$ 


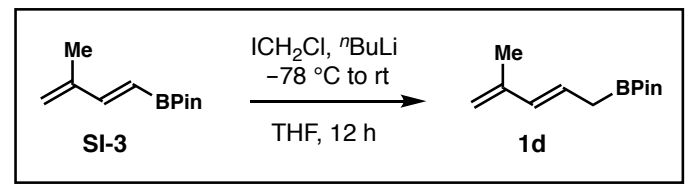

(E)-4,4,5,5-Tetramethyl-2-(4-methylpenta-2,4-dien-1-yl)-1,3,2-dioxaborolane

(1d) Prepared from SI- $\mathbf{3}^{3}$ according to the procedure for the synthesis of 1a. The crude mixture was purified by flash column chromatography (gradient elution with hexane and diethyl ether, 100:1 to 20:1) to give allylboronate 1d as colorless oil in $c a .72 \%$ yield (341 mg, contain $\sim 10 \% \mathrm{SI}-3) .{ }^{1} \mathrm{H}$ NMR $\left(400 \mathrm{MHz}, \mathrm{CDCl}_{3}\right) \delta 6.14(\mathrm{~d}, J=15.5 \mathrm{~Hz}, 1 \mathrm{H})$, $5.71(\mathrm{dt}, J=15.4,7.6 \mathrm{~Hz}, 1 \mathrm{H}), 4.81(\mathrm{~s}, 2 \mathrm{H}), 1.83(\mathrm{~s}, 3 \mathrm{H}), 1.76(\mathrm{~d}, J=7.5 \mathrm{~Hz}, 2 \mathrm{H}), 1.25$ (s, $12 \mathrm{H}) .{ }^{13} \mathrm{C}$ NMR $\left(101 \mathrm{MHz}, \mathrm{CDCl}_{3}\right) \delta 142.8,133.8,126.4,113.8,83.7,25.1,19.1$. HRMS $\left(\mathrm{ESI}^{+}\right): \mathrm{m} / z$ for $\mathrm{C}_{12} \mathrm{H}_{22} \mathrm{BO}_{2}[\mathrm{M}+\mathrm{H}]^{+}$calcd. 209.1713, found 209.1681.

\section{Syntheses of allylboronate reagent $1 \mathrm{~b}$ :}

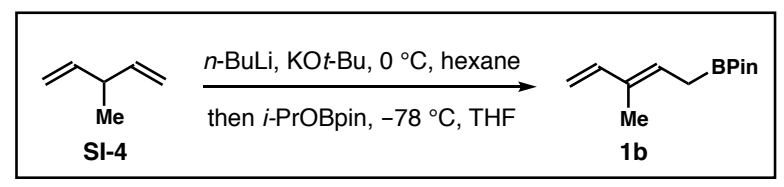

(E)-4,4,5,5-Tetramethyl-2-(3-methylpenta-2,4-dien-1-yl)-1,3,2-dioxaborolane $\quad(1 \mathrm{~b})^{2}$ To a suspension of $\mathrm{KO}^{t} \mathrm{Bu}\left(1.12 \mathrm{~g}, 10 \mathrm{mmol}, 1.0\right.$ equiv) and ${ }^{n} \mathrm{BuLi}(1.6 \mathrm{M}, 10.5 \mathrm{mmol}$, 1.05 equiv) in hexanes $(10 \mathrm{~mL})$ at $0{ }^{\circ} \mathrm{C}$ was added diene SI-4 $(0.82 \mathrm{~g}, 10 \mathrm{mmol}, 1.0$ equiv). After stirring vigorously at $0{ }^{\circ} \mathrm{C}$ for $20 \mathrm{~min}$, the hexane solvent was removed under reduced pressure. The reaction flask was placed in an acetone-dry ice bath and cooled to $-78{ }^{\circ} \mathrm{C}$. Then pre-cooled THF $\left(-78{ }^{\circ} \mathrm{C}, 50 \mathrm{~mL}\right)$ was added, followed by addition of ${ }^{i} \operatorname{PrOBP}$ in $(2 \mathrm{~mL}, 10 \mathrm{mmol}, 1.0$ equiv). The reaction mixture was kept stirring at $-78{ }^{\circ} \mathrm{C}$ for $1 \mathrm{~h}$. Then a solution of saturated $\mathrm{NH}_{4} \mathrm{Cl}$ was added to the reaction mixture. After warming to ambient temperature, the organic layer was separated and the aqueous layer was extracted with ethyl ether $(20 \mathrm{~mL} \times 3)$. The combined organic extracts were dried over anhydrous sodium sulfate, filtered, and concentrated under reduced pressure. Purification of the crude product was performed by flash column chromatography (gradient elution with hexane and diethyl ether, 100:1 to 20:1) to give boronate $\mathbf{1 b}$ as colorless oil (794 mg, 38\% yield). ${ }^{1} \mathrm{H}$ NMR (600 MHz, $\left.\mathrm{CDCl}_{3}\right) \delta 6.39$ (dd, $J=17.4,10.7$ $\mathrm{Hz}, 1 \mathrm{H}), 5.63$ (t, $J=8.1 \mathrm{~Hz}, 1 \mathrm{H}), 5.03(\mathrm{~d}, J=17.4 \mathrm{~Hz}, 1 \mathrm{H}), 4.87$ (d, $J=10.7 \mathrm{~Hz}, 1 \mathrm{H})$, $1.77(\mathrm{~d}, J=8.1 \mathrm{~Hz}, 2 \mathrm{H}), 1.71(\mathrm{~s}, 3 \mathrm{H}), 1.24(\mathrm{~s}, 12 \mathrm{H}) \cdot{ }^{13} \mathrm{C} \mathrm{NMR}\left(151 \mathrm{MHz}, \mathrm{CDCl}_{3}\right) \delta$ 142.0, 134.5, 128.3, 109.9, 83.6, 25.1, 13.3, 11.9. HRMS (EI $\left.{ }^{+}\right): m / z$ for $\mathrm{C}_{12} \mathrm{H}_{21} \mathrm{BO}_{2}[\mathrm{M}]^{+}$ calcd. 208.1635, found 208.1614 . 


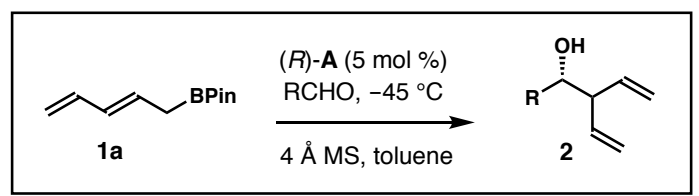

General procedure for the syntheses of homoallylic alcohols 2: To a reaction flask containing a stir bar and freshly activated $4 \AA \mathrm{MS}(50 \mathrm{mg})$ was added phosphoric acid $(R)$-A $(3.8 \mathrm{mg}, 0.005 \mathrm{mmol})$. Toluene $(0.3 \mathrm{~mL})$ was added to the flask followed by drop wise addition of freshly distilled aldehyde $(0.1 \mathrm{mmol}$, if it is a liquid). The mixture was placed in a $-45{ }^{\circ} \mathrm{C}$ cold bath and stirred for $5 \mathrm{~min}$. Then allylboronate $1 \mathrm{a}(0.13 \mathrm{mmol})$ was added slowly to the reaction mixture via a microliter syringe. The mixture was kept at $-45{ }^{\circ} \mathrm{C}$ and stirred for $48 \mathrm{~h}$. After complete consumption of the aldehyde, sat. $\mathrm{NaHCO}_{3}$ $(1.0 \mathrm{~mL})$ was added to the reaction mixture followed by slow addition of $30 \% \mathrm{H}_{2} \mathrm{O}_{2}(0.5$ $\mathrm{mL})$ at $0{ }^{\circ} \mathrm{C}$. The reaction was stirred vigorously for $3 \mathrm{~h}$; then diethyl ether $(1 \mathrm{~mL})$ and brine $(1 \mathrm{~mL})$ was added. The organic layer was separated and the aqueous layer was extracted with $\mathrm{Et}_{2} \mathrm{O}(2 \mathrm{~mL} \times 3)$. The combined organic extracts were dried over anhydrous sodium sulfate, filtered, and concentrated under reduced pressure. Purification of the crude product was performed by flash chromatography (gradient elution with hexane and ethyl acetate) to give product 2.

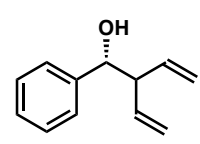

(R)-1-Phenyl-2-vinylbut-3-en-1-ol (2a) ${ }^{4}$ Prepared according to the general procedure. The crude mixture was purified by flash column chromatography (gradient elution with hexane and ethyl acetate, 20:1 to 10:1) to give compound 2a as colorless oil in $75 \%$ yield $(13.1 \mathrm{mg})$. The enantiomeric excess was determined by HPLC analysis to be $97 \%$ ee $\left(254 \mathrm{~nm}, 25^{\circ} \mathrm{C}\right) ; \mathrm{t}_{1}=5.98 \mathrm{~min}, \mathrm{t}_{2}=6.66 \mathrm{~min}$ [(Chiralpak IC) hexane $/ i-\mathrm{PrOH}, 95: 5,1.0 \mathrm{~mL} / \mathrm{min}] ;[\alpha]_{\mathrm{D}}{ }^{25}=59.1^{\circ}$ (c 0.43, $\left.\mathrm{CHCl}_{3}\right)$; Colorless oil; ${ }^{1} \mathrm{H}$ NMR $\left(600 \mathrm{MHz}, \mathrm{CDCl}_{3}\right) \delta 7.36-7.31(\mathrm{~m}, 4 \mathrm{H}), 7.26-7.29(\mathrm{~m}, 1 \mathrm{H})$, 5.85 (ddd, $J=17.6,10.1,8.5 \mathrm{~Hz}, 1 \mathrm{H}), 5.67$ (ddd, $J=17.4,10.4,7.2 \mathrm{~Hz}, 1 \mathrm{H}), 5.25$ (d, $J=$ $10.3 \mathrm{~Hz}, 1 \mathrm{H}), 5.20(\mathrm{~d}, J=17.2 \mathrm{~Hz}, 1 \mathrm{H}), 5.05(\mathrm{~d}, J=10.4 \mathrm{~Hz}, 1 \mathrm{H}), 5.01(\mathrm{~d}, J=17.3 \mathrm{~Hz}$, $1 \mathrm{H}), 4.59$ (d, $J=7.2 \mathrm{~Hz}, 1 \mathrm{H}), 3.11$ (app. q, $J=7.5 \mathrm{~Hz}, 1 \mathrm{H}), 2.21$ (br, 1H). ${ }^{13} \mathrm{C}$ NMR (151 $\left.\mathrm{MHz}, \mathrm{CDCl}_{3}\right) \delta 142.0,137.1,137.0,128.5,128.0,127.2,118.8,117.5,76.5,56.6$. A 1 mmol scale reaction was also conducted to give $\mathbf{2 a}$ in $83 \%$ yield (144 mg).

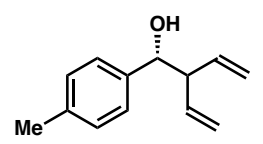

(R)-1-(p-Tolyl)-2-vinylbut-3-en-1-ol (2b) Prepared according to the general procedure. The crude mixture was purified by flash column chromatography (gradient elution with hexane and ethyl acetate, 20:1 to $10: 1)$ to give compound $\mathbf{2 b}$ as colorless oil in $98 \%$ yield $(18.5 \mathrm{mg})$. The enantiomeric 
excess was determined by HPLC analysis to be $99 \%$ ee $\left(254 \mathrm{~nm}, 25^{\circ} \mathrm{C}\right) ; \mathrm{t}_{1}=8.59 \mathrm{~min}, \mathrm{t}_{2}$ $=9.24 \mathrm{~min}\left[(\right.$ Chiralpak IG) hexane $/ i-\mathrm{PrOH}, 97: 3,1.0 \mathrm{~mL} / \mathrm{min}] ;[\alpha]_{\mathrm{D}}{ }^{25}=87.3^{\circ}(c 0.60$, $\left.\mathrm{CHCl}_{3}\right) ;{ }^{1} \mathrm{H} \mathrm{NMR}\left(600 \mathrm{MHz}, \mathrm{CDCl}_{3}\right) \delta 7.21(\mathrm{~d}, J=7.9 \mathrm{~Hz}, 2 \mathrm{H}), 7.15(\mathrm{~d}, J=7.9 \mathrm{~Hz}, 2 \mathrm{H})$, 5.85 (ddd, $J=17.4,10.2,8.4 \mathrm{~Hz}, 1 \mathrm{H}$ ), 5.67 (ddd, $J=17.4,10.4,7.1 \mathrm{~Hz}, 1 \mathrm{H}), 5.24$ (d, $J=$ $10.3 \mathrm{~Hz}, 1 \mathrm{H}), 5.20$ (d, $J=17.2 \mathrm{~Hz}, 1 \mathrm{H}), 5.04$ (d, $J=10.4 \mathrm{~Hz}, 1 \mathrm{H}), 5.01$ (d, $J=17.3 \mathrm{~Hz}$, 1H), 4.55 (dd, $J=7.3,2.9 \mathrm{~Hz}, 1 \mathrm{H}$ ), 3.10 (app. q, $J=7.5 \mathrm{~Hz}, 1 \mathrm{H}$ ), 2.34 (s, 3H), 2.17 (d, $J$ $=2.9 \mathrm{~Hz}, 1 \mathrm{H}) .{ }^{13} \mathrm{C} \mathrm{NMR}\left(151 \mathrm{MHz}, \mathrm{CDCl}_{3}\right) \delta 139.0,137.7,137.3,137.1,129.2,127.1$, 118.7, 117.4, 76.3, 56.5, 21.5. HRMS ( $\left.\mathrm{API}^{+}\right): m / z$ for $\mathrm{C}_{13} \mathrm{H}_{15}[\mathrm{M}-\mathrm{OH}]^{+}$calcd. 171.1174, found 171.1182 .

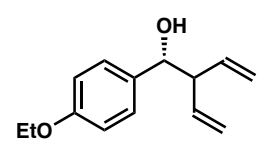

(R)-1-(4-Ethoxyphenyl)-2-vinylbut-3-en-1-ol (2c) Prepared according to the general procedure. The crude mixture was purified by flash column chromatography (gradient elution with hexane and ethyl acetate, $20: 1$ to $10: 1)$ to give compound $\mathbf{2 c}$ as colorless oil in $93 \%$ yield $(20.3 \mathrm{mg})$. The enantiomeric excess was determined by HPLC analysis to be $99 \%$ ee $\left(254 \mathrm{~nm}, 25^{\circ} \mathrm{C}\right)$; $\mathrm{t}_{1}$ $=7.65 \mathrm{~min}, \mathrm{t}_{2}=8.82 \mathrm{~min}\left[(\right.$ Chiralpak IB) hexane $/ i-\operatorname{PrOH}, 97: 3,1.0 \mathrm{~mL} / \mathrm{min}] ;[\alpha]_{\mathrm{D}}{ }^{25}=$ $62.5^{\circ}$ (c 0.70, $\left.\mathrm{CHCl}_{3}\right) ;{ }^{1} \mathrm{H}$ NMR $\left(600 \mathrm{MHz}, \mathrm{CDCl}_{3}\right) \delta 7.22(\mathrm{~d}, J=8.5 \mathrm{~Hz}, 2 \mathrm{H}), 6.86(\mathrm{~d}, J$ $=8.5 \mathrm{~Hz}, 2 \mathrm{H}), 5.85(\mathrm{ddd}, J=17.2,10.0,8.6 \mathrm{~Hz}, 1 \mathrm{H}), 5.64(\mathrm{ddd}, J=17.4,10.4,7.1 \mathrm{~Hz}$, $1 \mathrm{H}), 5.25(\mathrm{~d}, J=10.3 \mathrm{~Hz}, 1 \mathrm{H}), 5.20(\mathrm{~d}, J=17.2 \mathrm{~Hz}, 1 \mathrm{H}), 5.03(\mathrm{~d}, J=10.4 \mathrm{~Hz}, 1 \mathrm{H}), 4.99$ (d, $J=17.3 \mathrm{~Hz}, 1 \mathrm{H}), 4.51(\mathrm{dd}, J=7.5,2.5 \mathrm{~Hz}, 1 \mathrm{H}), 4.02$ (q, $J=7.0 \mathrm{~Hz}, 2 \mathrm{H}), 3.08$ (app. q, $J=7.6 \mathrm{~Hz}, 1 \mathrm{H}), 2.16(\mathrm{~d}, J=2.8 \mathrm{~Hz}, 1 \mathrm{H}), 1.41(\mathrm{t}, J=7.0 \mathrm{~Hz}, 3 \mathrm{H}) .{ }^{13} \mathrm{C} \mathrm{NMR}(151 \mathrm{MHz}$, $\left.\mathrm{CDCl}_{3}\right) \delta 158.7,137.4,137.1,134.0,128.4,118.7,117.3,114.3,76.1,63.7,56.6,15.2$. HRMS ( $\mathrm{API}^{+}$): $m / z$ for $\mathrm{C}_{14} \mathrm{H}_{17} \mathrm{O}[\mathrm{M}-\mathrm{OH}]^{+}$calcd. 201.1279, found 201.1286.

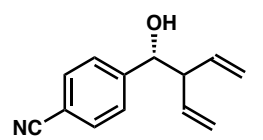

(R)-4-(1-Hydroxy-2-vinylbut-3-en-1-yl)benzonitrile (2d) Prepared according to the general procedure. The crude mixture was purified by flash column chromatography (gradient elution with hexane and ethyl acetate, $20: 1$ to $5: 1)$ to give compound $\mathbf{2 d}$ as colorless oil in $96 \%$ yield $(19.1 \mathrm{mg})$. The enantiomeric excess was determined by HPLC analysis to be $95 \%$ ee $\left(254 \mathrm{~nm}, 25^{\circ} \mathrm{C}\right) ; \mathrm{t}_{1}$ $=20.1 \mathrm{~min}, \mathrm{t}_{2}=21.8 \mathrm{~min}[($ Chiralpak IA $)$ hexane $/ i-\operatorname{PrOH}, 97: 3,1.0 \mathrm{~mL} / \mathrm{min}] ;[\alpha]_{\mathrm{D}}{ }^{25}=$ $75.4^{\circ}\left(c\right.$ 0.65, $\left.\mathrm{CHCl}_{3}\right){ }^{1} \mathrm{H}_{\mathrm{NMR}}\left(600 \mathrm{MHz}, \mathrm{CDCl}_{3}\right) \delta 7.61(\mathrm{~d}, J=8.2 \mathrm{~Hz}, 2 \mathrm{H}), 7.42(\mathrm{~d}, J$ $=8.2 \mathrm{~Hz}, 2 \mathrm{H}), 5.79(\mathrm{ddd}, J=17.5,10.2,8.5 \mathrm{~Hz}, 1 \mathrm{H}), 5.67(\mathrm{ddd}, J=17.4,10.4,7.4 \mathrm{~Hz}$, $1 \mathrm{H}), 5.24(\mathrm{~d}, J=10.2 \mathrm{~Hz}, 1 \mathrm{H}), 5.15(\mathrm{~d}, J=17.2 \mathrm{~Hz}, 1 \mathrm{H}), 5.09(\mathrm{~d}, J=10.4 \mathrm{~Hz}, 1 \mathrm{H}), 5.00$ (d, $J=17.2 \mathrm{~Hz}, 1 \mathrm{H}$ ), 4.65 (dd, $J=6.7,1.4 \mathrm{~Hz}, 1 \mathrm{H}$ ), 3.03 (app. q, $J=7.4 \mathrm{~Hz}, 1 \mathrm{H}$ ), 2.34 (br, $1 \mathrm{H}) .{ }^{13} \mathrm{C} \mathrm{NMR}\left(151 \mathrm{MHz}, \mathrm{CDCl}_{3}\right) \delta 147.3,136.1,135.9,132.2,127.9,119.7,119.2$, 
118.4, 111.6, 75.6, 56.8. HRMS $\left(\mathrm{ESI}^{+}\right): \mathrm{m} / z$ for $\mathrm{C}_{13} \mathrm{H}_{13} \mathrm{NONa}[\mathrm{M}+\mathrm{Na}]^{+}$calcd. 222.0895, found 222.0907 .

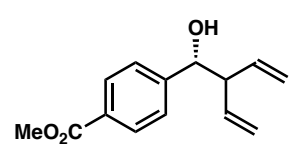

Methyl (R)-4-(1-hydroxy-2-vinylbut-3-en-1-yl)benzoate

Prepared according to the general procedure. The crude mixture was purified by flash column chromatography (gradient elution with hexane and ethyl acetate, 20:1 to 5:1) to give compound 2 e as colorless oil in $95 \%$ yield $(22.1 \mathrm{mg})$. The enantiomeric excess was determined by HPLC analysis to be $95 \%$ ee (254 $\left.\mathrm{nm}, 25{ }^{\circ} \mathrm{C}\right) ; \mathrm{t}_{1}=7.32 \mathrm{~min}, \mathrm{t}_{2}=7.92 \mathrm{~min}[($ Chiralpak IC) hexane $/ i-\mathrm{PrOH}, 97: 3,1.0$ $\mathrm{mL} / \mathrm{min}] ;[\alpha]_{\mathrm{D}}{ }^{25}=42.8^{\circ}\left(c 0.80, \mathrm{CHCl}_{3}\right) ;{ }^{1} \mathrm{H} \mathrm{NMR}\left(600 \mathrm{MHz}, \mathrm{CDCl}_{3}\right) \delta 8.00(\mathrm{~d}, J=8.1$ $\mathrm{Hz}, 2 \mathrm{H}), 7.39$ (d, $J=8.1 \mathrm{~Hz}, 2 \mathrm{H}), 5.82$ (ddd, $J=17.7,10.1,8.4 \mathrm{~Hz}, 1 \mathrm{H}), 5.67$ (ddd, $J=$ $17.4,10.3,7.3 \mathrm{~Hz}, 1 \mathrm{H}), 5.25$ (d, $J=10.3 \mathrm{~Hz}, 1 \mathrm{H}), 5.17$ (d, $J=17.2 \mathrm{~Hz}, 1 \mathrm{H}), 5.06$ (d, $J=$ $10.4 \mathrm{~Hz}, 1 \mathrm{H}), 5.00(\mathrm{~d}, J=17.2 \mathrm{~Hz}, 1 \mathrm{H}), 4.66(\mathrm{dd}, J=6.8,2.9 \mathrm{~Hz}, 1 \mathrm{H}), 3.91(\mathrm{~s}, 3 \mathrm{H}), 3.08$ (app. q, $J=7.4 \mathrm{~Hz}, 1 \mathrm{H}), 2.30(\mathrm{~d}, J=2.9 \mathrm{~Hz}, 1 \mathrm{H}) .{ }^{13} \mathrm{C} \mathrm{NMR}\left(151 \mathrm{MHz}, \mathrm{CDCl}_{3}\right) \delta 167.3$, 147.1, 136.5, 136.4, 129.74, 129.67, 127.2, 119.3, 118.0, 76.0, 56.7, 52.5. HRMS (API $\left.{ }^{+}\right)$: $m / z$ for $\mathrm{C}_{14} \mathrm{H}_{16} \mathrm{O}_{3} \mathrm{Na}[\mathrm{M}+\mathrm{Na}]^{+}$calcd. 255.0997, found 255.0998.

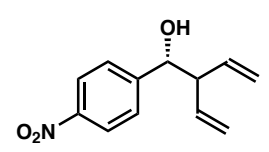

(R)-1-(4-Nitrophenyl)-2-vinylbut-3-en-1-ol (2f) Prepared according to the general procedure. The crude mixture was purified by flash column chromatography (gradient elution with hexane and ethyl acetate, 20:1 to $5: 1)$ to give compound $2 \mathbf{f}$ as colorless oil in $92 \%$ yield $(20.2 \mathrm{mg})$. The enantiomeric excess was determined by HPLC analysis to be $94 \%$ ee $\left(254 \mathrm{~nm}, 25^{\circ} \mathrm{C}\right) ; \mathrm{t}_{1}=6.44 \mathrm{~min}, \mathrm{t}_{2}$ $=6.86 \mathrm{~min}\left[(\right.$ Chiralpak ID) hexane $/ \mathrm{i}-\mathrm{PrOH}, 97: 3,1.0 \mathrm{~mL} / \mathrm{min}] ;[\alpha]_{\mathrm{D}}{ }^{25}=67.7^{\circ}$ (c 0.70 , $\mathrm{CHCl}_{3}$ ); ${ }^{1} \mathrm{H} \mathrm{NMR}\left(400 \mathrm{MHz}, \mathrm{CDCl}_{3}\right) \delta 8.19(\mathrm{~d}, J=8.6 \mathrm{~Hz}, 2 \mathrm{H}), 7.49$ (d, $\left.J=8.5 \mathrm{~Hz}, 2 \mathrm{H}\right)$, 5.82 (ddd, $J=17.6,9.9,8.8 \mathrm{~Hz}, 1 \mathrm{H}), 5.70(\mathrm{ddd}, J=17.5,10.4,7.4 \mathrm{~Hz}, 1 \mathrm{H}), 5.26(\mathrm{~d}, J=$ $10.2 \mathrm{~Hz}, 1 \mathrm{H}), 5.16(\mathrm{~d}, J=17.2 \mathrm{~Hz}, 1 \mathrm{H}), 5.11(\mathrm{~d}, J=10.4 \mathrm{~Hz}, 1 \mathrm{H}), 5.02(\mathrm{~d}, J=17.2 \mathrm{~Hz}$, $1 \mathrm{H}), 4.73(\mathrm{dd}, J=6.5,2.7 \mathrm{~Hz}, 1 \mathrm{H}), 3.06$ (app. q, $J=7.4 \mathrm{~Hz}, 1 \mathrm{H}), 2.36$ (d, $J=3.0 \mathrm{~Hz}, 1 \mathrm{H})$. ${ }^{13} \mathrm{C}$ NMR $\left(151 \mathrm{MHz}, \mathrm{CDCl}_{3}\right) \delta 149.4,147.6,136.0,135.8,128.0,123.6,119.8,118.6$, 75.4, 56.9. HRMS $\left(\mathrm{ESI}^{+}\right): \mathrm{m} / z$ for $\mathrm{C}_{12} \mathrm{H}_{13} \mathrm{NO}_{3} \mathrm{Na}[\mathrm{M}+\mathrm{Na}]^{+}$calcd. 242.0793, found 242.0815 .

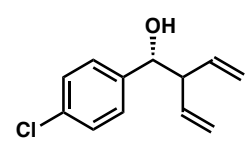

(R)-1-(4-Chlorophenyl)-2-vinylbut-3-en-1-ol (2g) Prepared according to the general procedure. The crude mixture was purified by flash column chromatography (gradient elution with hexane and ethyl acetate, 20:1 to $10: 1)$ to give compound $\mathbf{2} \mathrm{g}$ as colorless oil in $90 \%$ yield $(18.8 \mathrm{mg})$. The enantiomeric excess was determined by HPLC analysis to be $91 \%$ ee $\left(254 \mathrm{~nm}, 25^{\circ} \mathrm{C}\right) ; \mathrm{t}_{1}=5.38 \mathrm{~min}, \mathrm{t}_{2}$ 
$=5.92 \mathrm{~min}\left[(\right.$ Chiralpak IB) hexane $/ i-\mathrm{PrOH}, 97: 3,1.0 \mathrm{~mL} / \mathrm{min}] ;[\alpha]_{\mathrm{D}}{ }^{25}=92.0^{\circ}(c 0.65$, $\left.\mathrm{CHCl}_{3}\right) ;{ }^{1} \mathrm{H}$ NMR $\left(600 \mathrm{MHz}, \mathrm{CDCl}_{3}\right) \delta 7.31(\mathrm{~d}, J=8.3 \mathrm{~Hz}, 2 \mathrm{H}), 7.25(\mathrm{~d}, J=8.7 \mathrm{~Hz}, 2 \mathrm{H})$, 5.82 (ddd, $J=17.7,10.2,8.8 \mathrm{~Hz}, 1 \mathrm{H}$ ), 5.65 (ddd, $J=17.4,10.4,7.3 \mathrm{~Hz}, 1 \mathrm{H}$ ), 5.25 (d, $J=$ $10.3 \mathrm{~Hz}, 1 \mathrm{H}), 5.19$ (d, $J=17.2 \mathrm{~Hz}, 1 \mathrm{H}), 5.06$ (d, $J=10.4 \mathrm{~Hz}, 1 \mathrm{H}), 5.00$ (d, $J=17.3 \mathrm{~Hz}$, $1 \mathrm{H}$ ), 4.57 (dd, $J=7.1,2.7 \mathrm{~Hz}, 1 \mathrm{H}$ ), 3.04 (app. q, $J=7.5 \mathrm{~Hz}, 1 \mathrm{H}$ ), 2.23 (d, $J=2.9 \mathrm{~Hz}, 1 \mathrm{H}$ ). ${ }^{13} \mathrm{C}$ NMR $\left(151 \mathrm{MHz}, \mathrm{CDCl}_{3}\right) \delta 140.4,136.7,136.5,133.6,128.60,128.56,119.2,117.9$, 75.7, 56.7. HRMS $\left(\mathrm{ESI}^{+}\right): m / z$ for $\mathrm{C}_{12} \mathrm{H}_{12} \mathrm{Cl}[\mathrm{M}-\mathrm{OH}]^{+}$calcd. 191.0628, found 191.0635.

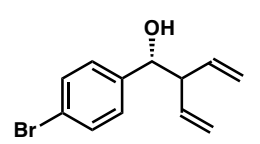

(R)-1-(4-Bromophenyl)-2-vinylbut-3-en-1-ol (2h) Prepared according to the general procedure. The crude mixture was purified by flash column chromatography (gradient elution with hexane and ethyl acetate, 20:1 to 10:1) to give compound $\mathbf{2 h}$ as colorless oil in $96 \%$ yield $(24.3 \mathrm{mg})$. The enantiomeric excess was determined by HPLC analysis to be $93 \%$ ee $\left(254 \mathrm{~nm}, 25^{\circ} \mathrm{C}\right) ; \mathrm{t}_{1}=4.27 \mathrm{~min}, \mathrm{t}_{2}$ $=4.57 \mathrm{~min}\left[(\right.$ Chiralpak IB) hexane $/ i-\mathrm{PrOH}, 97: 3,1.0 \mathrm{~mL} / \mathrm{min}] ;[\alpha]_{\mathrm{D}}{ }^{25}=63.8^{\circ}(c 0.90$, $\left.\mathrm{CHCl}_{3}\right) ;{ }^{1} \mathrm{H} \mathrm{NMR}\left(600 \mathrm{MHz}, \mathrm{CDCl}_{3}\right) \delta 7.46(\mathrm{~d}, J=8.2 \mathrm{~Hz}, 2 \mathrm{H}), 7.19(\mathrm{~d}, J=8.3 \mathrm{~Hz}, 2 \mathrm{H})$, 5.82 (ddd, $J=17.7,10.0,8.6 \mathrm{~Hz}, 1 \mathrm{H}), 5.65$ (ddd, $J=17.4,10.4,7.3 \mathrm{~Hz}, 1 \mathrm{H}), 5.25$ (d, $J=$ $10.3 \mathrm{~Hz}, 1 \mathrm{H}), 5.18$ (d, $J=17.2 \mathrm{~Hz}, 1 \mathrm{H}), 5.06$ (d, $J=10.4 \mathrm{~Hz}, 1 \mathrm{H}), 5.00$ (d, $J=17.3 \mathrm{~Hz}$, $1 \mathrm{H}), 4.55$ (dd, $J=7.1,2.7 \mathrm{~Hz}, 1 \mathrm{H}$ ), 3.04 (app. q, $J=7.5 \mathrm{~Hz}, 1 \mathrm{H}$ ), 2.23 (d, $J=2.8 \mathrm{~Hz}, 1 \mathrm{H}$ ). ${ }^{13} \mathrm{C}$ NMR $\left(151 \mathrm{MHz}, \mathrm{CDCl}_{3}\right) \delta 141.0,136.6,136.5,131.5,128.9,121.8,119.2,117.9$, 75.8, 56.7. HRMS $\left(\mathrm{ESI}^{+}\right): m / z$ for $\mathrm{C}_{12} \mathrm{H}_{12} \mathrm{Br}[\mathrm{M}-\mathrm{OH}]^{+}$calcd. 235.0122, found 235.0130.

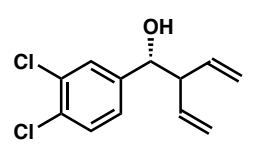

\section{(R)-1-(3,4-Dichlorophenyl)-2-vinylbut-3-en-1-ol}

(2i) Prepared according to the general procedure. The crude mixture was purified by flash column chromatography (gradient elution with hexane and ethyl acetate, $20: 1$ to $10: 1$ ) to give compound $\mathbf{2 i}$ as colorless oil in $93 \%$ yield $(22.6 \mathrm{mg})$. The enantiomeric excess was determined by HPLC analysis to be $95 \%$ ee $\left(254 \mathrm{~nm}, 25^{\circ} \mathrm{C}\right)$; $\mathrm{t}_{1}$ $=9.30 \mathrm{~min}, \mathrm{t}_{2}=10.0 \mathrm{~min}\left[(\right.$ Chiralpak IA) hexane $/ i-\operatorname{PrOH}, 97: 3,1.0 \mathrm{~mL} / \mathrm{min}] ;[\alpha]_{\mathrm{D}}{ }^{25}=$ $50.7^{\circ}\left(c 0.90, \mathrm{CHCl}_{3}\right) ;{ }^{1} \mathrm{H} \mathrm{NMR}\left(600 \mathrm{MHz}, \mathrm{CDCl}_{3}\right) \delta 7.42(\mathrm{~d}, J=1.6 \mathrm{~Hz}, 1 \mathrm{H}), 7.40(\mathrm{~d}, J$ $=8.3 \mathrm{~Hz}, 1 \mathrm{H}), 7.14(\mathrm{dd}, J=8.2,1.6 \mathrm{~Hz}, 1 \mathrm{H}), 5.80(\mathrm{ddd}, J=17.5,10.1,8.6 \mathrm{~Hz}, 1 \mathrm{H}), 5.66$ (ddd, $J=17.4,10.4,7.3 \mathrm{~Hz}, 1 \mathrm{H}), 5.27(\mathrm{~d}, J=10.3 \mathrm{~Hz}, 1 \mathrm{H}), 5.19$ (d, $J=17.2 \mathrm{~Hz}, 1 \mathrm{H}$ ), $5.10(\mathrm{~d}, J=10.4 \mathrm{~Hz}, 1 \mathrm{H}), 5.02(\mathrm{~d}, J=17.2 \mathrm{~Hz}, 1 \mathrm{H}), 4.55(\mathrm{dd}, J=7.0,2.8 \mathrm{~Hz}, 1 \mathrm{H}), 3.02$ (app. q, $J=7.5 \mathrm{~Hz}, 1 \mathrm{H}), 2.26(\mathrm{~d}, J=2.9 \mathrm{~Hz}, 1 \mathrm{H}) .{ }^{13} \mathrm{C} \mathrm{NMR}\left(151 \mathrm{MHz}, \mathrm{CDCl}_{3}\right) \delta 142.3$, 136.20, 136.18, 132.5, 131.7, 130.3, 129.1, 126.6, 119.6, 118.3, 75.2, 56.7. HRMS (ESI $\left.{ }^{+}\right)$: $m / z$ for $\mathrm{C}_{12} \mathrm{H}_{11} \mathrm{Cl}_{2}[\mathrm{M}-\mathrm{OH}]^{+}$calcd. 225.0238, found 225.0235. 


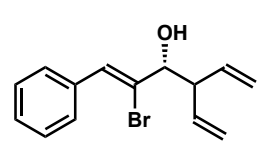

(R,Z)-2-Bromo-1-phenyl-4-vinylhexa-1,5-dien-3-ol (2j) Prepared according to the general procedure. The crude mixture was purified by flash column chromatography (gradient elution with hexane and ethyl acetate, $20: 1$ to $10: 1)$ to give compound $\mathbf{2} \mathbf{j}$ as colorless oil in $92 \%$ yield $(25.7 \mathrm{mg})$. The enantiomeric excess was determined by HPLC analysis to be $93 \%$ ee $\left(254 \mathrm{~nm}, 25{ }^{\circ} \mathrm{C}\right) ; \mathrm{t}_{1}$ $=5.49 \mathrm{~min}, \mathrm{t}_{2}=5.87 \mathrm{~min}\left[(\right.$ Chiralpak IC) hexane $/ i-\mathrm{PrOH}, 97: 3,1.0 \mathrm{~mL} / \mathrm{min}] ;[\alpha]_{\mathrm{D}}{ }^{25}=$ $-4.6^{\circ}\left(c 0.95, \mathrm{CHCl}_{3}\right) ;{ }^{1} \mathrm{H} \mathrm{NMR}\left(600 \mathrm{MHz}, \mathrm{CDCl}_{3}\right) \delta 7.59(\mathrm{~d}, J=7.6 \mathrm{~Hz}, 2 \mathrm{H}), 7.37(\mathrm{dd}, J$ $=7.7,7.4 \mathrm{~Hz}, 2 \mathrm{H}), 7.31(\mathrm{dd}, J=7.2,7.2 \mathrm{~Hz}, 1 \mathrm{H}), 7.04(\mathrm{~s}, 1 \mathrm{H}), 5.91$ (ddd, $J=17.5,10.1$, $8.3 \mathrm{~Hz}, 1 \mathrm{H}), 5.79$ (ddd, $J=17.5,10.2,7.5 \mathrm{~Hz}, 1 \mathrm{H}), 5.29$ (d, $J=10.9 \mathrm{~Hz}, 1 \mathrm{H}), 5.27$ (d, $J=$ $18.0 \mathrm{~Hz}, 1 \mathrm{H}), 5.20(\mathrm{~d}, J=17.2 \mathrm{~Hz}, 1 \mathrm{H}), 5.16(\mathrm{~d}, J=10.4 \mathrm{~Hz}, 1 \mathrm{H}), 4.16$ (dd, $J=7.4,5.2$ $\mathrm{Hz}, 1 \mathrm{H}), 3.31$ (app. q, $J=7.7 \mathrm{~Hz}, 1 \mathrm{H}), 2.24(\mathrm{~d}, J=5.0 \mathrm{~Hz}, 1 \mathrm{H}) .{ }^{13} \mathrm{C}$ NMR $(151 \mathrm{MHz}$, $\left.\mathrm{CDCl}_{3}\right) \delta 136.3,136.0,135.3,130.3,129.5,128.53,128.48,127.9,119.3,117.7,79.2$, 53.1. HRMS (ESI $\left.{ }^{+}\right): m / z$ for $\mathrm{C}_{14} \mathrm{H}_{15} \mathrm{ONaBr}[\mathrm{M}+\mathrm{Na}]^{+}$calcd. 301.0204, found 301.0220.

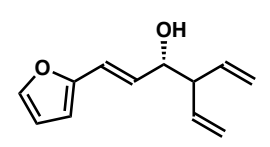

(S,E)-1-(Furan-2-yl)-4-vinylhexa-1,5-dien-3-ol

(2k) Prepared according to the general procedure. The crude mixture was purified by flash column chromatography (gradient elution with hexane and ethyl acetate, $20: 1$ to $10: 1)$ to give compound $\mathbf{2 k}$ as colorless oil in $94 \%$ yield (17.9 $\mathrm{mg})$. The enantiomeric excess was determined by HPLC analysis to be $96 \%$ ee $\left(254 \mathrm{~nm}, 25{ }^{\circ} \mathrm{C}\right) ; \mathrm{t}_{1}$ $=8.14 \mathrm{~min}, \mathrm{t}_{2}=8.78 \mathrm{~min}\left[(\right.$ Chiralpak IB) hexane $/ i-\mathrm{PrOH}, 97: 3,1.0 \mathrm{~mL} / \mathrm{min}] ;[\alpha]_{\mathrm{D}}{ }^{25}=$ $31.7^{\circ}\left(c 0.60, \mathrm{CHCl}_{3}\right) ;{ }^{1} \mathrm{H}$ NMR $\left(600 \mathrm{MHz}, \mathrm{CDCl}_{3}\right) \delta 7.34$ (app. s, $\left.1 \mathrm{H}\right), 6.44(\mathrm{~d}, J=15.8$ $\mathrm{Hz}, 1 \mathrm{H}), 6.36-6.37(\mathrm{~m}, 1 \mathrm{H}), 6.23(\mathrm{~d}, J=3.1 \mathrm{~Hz}, 1 \mathrm{H}), 6.18(\mathrm{dd}, J=15.8,6.1 \mathrm{~Hz}, 1 \mathrm{H})$, $5.81-5.89(\mathrm{~m}, 2 \mathrm{H}), 5.24(\mathrm{~d}, J=10.3 \mathrm{~Hz}, 1 \mathrm{H}), 5.16-5.21(\mathrm{~m}, 3 \mathrm{H}), 4.23$ (app. q, $J=5.6$ $\mathrm{Hz}, 1 \mathrm{H}), 2.99$ (ddd, J = 7.4, 7.4, 7.0 Hz, 1H), 1.88 (d, $J=4.9 \mathrm{~Hz}, 1 \mathrm{H}) .{ }^{13} \mathrm{C} \mathrm{NMR}(151$ $\left.\mathrm{MHz}, \mathrm{CDCl}_{3}\right) \delta 152.6,142.3,136.8,136.6,128.3,120.0,118.4,118.2,111.6,108.5,74.2$, 55.3. HRMS (ESI $\left.{ }^{+}\right): m / z$ for $\mathrm{C}_{12} \mathrm{H}_{13} \mathrm{O}[\mathrm{M}-\mathrm{OH}]^{+}$calcd. 173.0966, found 173.0958.

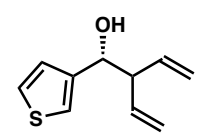

(R)-1-(Thiophen-3-yl)-2-vinylbut-3-en-1-ol (2l) Prepared according to the general procedure. The crude mixture was purified by flash column chromatography (gradient elution with hexane and ethyl acetate, 20:1 to $10: 1)$ to give compound $\mathbf{2 l}$ as colorless oil in $96 \%$ yield $(17.3 \mathrm{mg})$. The enantiomeric excess was determined by HPLC analysis to be $98 \%$ ee $\left(254 \mathrm{~nm}, 25{ }^{\circ} \mathrm{C}\right) ; \mathrm{t}_{1}=5.10 \mathrm{~min}, \mathrm{t}_{2}$ $=5.77 \mathrm{~min}$ [(Chiralpak IC) hexane $/ i-\mathrm{PrOH}, 97: 3,1.0 \mathrm{~mL} / \mathrm{min}] ;[\alpha]_{\mathrm{D}}{ }^{25}=-77.7^{\circ}$ (c 0.60, $\left.\mathrm{CHCl}_{3}\right) ;{ }^{1} \mathrm{H} \mathrm{NMR}\left(600 \mathrm{MHz}, \mathrm{CDCl}_{3}\right) \delta 7.29(\mathrm{dd}, J=4.9,3.1 \mathrm{~Hz}, 1 \mathrm{H}), 7.19(\mathrm{~d}, J=2.3 \mathrm{~Hz}$, $1 \mathrm{H}), 7.07$ (d, $J=5.0 \mathrm{~Hz}, 1 \mathrm{H}), 5.84$ (ddd, $J=17.5,10.2,8.2 \mathrm{~Hz}, 1 \mathrm{H}), 5.72$ (ddd, $J=17.4$, 10.4, 7.2 Hz, 1H), 5.25 (d, $J=10.3 \mathrm{~Hz}, 1 \mathrm{H}), 5.20(\mathrm{~d}, J=17.2 \mathrm{~Hz}, 1 \mathrm{H}), 5.10$ (d, $J=10.4$ 
$\mathrm{Hz}, 1 \mathrm{H}), 5.07$ (d, $J=17.3 \mathrm{~Hz}, 1 \mathrm{H}), 4.72(\mathrm{dd}, J=6.8,3.7 \mathrm{~Hz}, 1 \mathrm{H}), 3.13$ (app. q, $J=7.4$ $\mathrm{Hz}, 1 \mathrm{H}), 2.18(\mathrm{~d}, J=3.6 \mathrm{~Hz}, 1 \mathrm{H}) .{ }^{13} \mathrm{C} \mathrm{NMR}\left(151 \mathrm{MHz}, \mathrm{CDCl}_{3}\right) \delta 143.5,137.0,136.9$, $126.4,126.1,122.2,118.8,117.7,72.7,55.9$. HRMS $\left(\mathrm{ESI}^{+}\right): m / z$ for $\mathrm{C}_{10} \mathrm{H}_{11} \mathrm{~S}[\mathrm{M}-\mathrm{OH}]^{+}$ calcd. 163.0581, found 163.0575 .

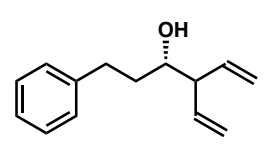

(S)-1-Phenyl-4-vinylhex-5-en-3-ol (2m) Prepared according to the general procedure. The crude mixture was purified by flash column chromatography (gradient elution with hexane and ethyl acetate, 20:1 to $10: 1)$ to give compound $\mathbf{2} \mathbf{m}$ as colorless oil in $88 \%$ yield $(17.8 \mathrm{mg})$. The enantiomeric excess was determined by HPLC analysis to be $91 \%$ ee $\left(254 \mathrm{~nm}, 25^{\circ} \mathrm{C}\right) ; \mathrm{t}_{1}=6.59 \mathrm{~min}, \mathrm{t}_{2}$ $=9.13 \mathrm{~min}\left[(\right.$ Chiralpak IC) hexane $/ i-\mathrm{PrOH}, 97: 3,1.0 \mathrm{~mL} / \mathrm{min}] ;[\alpha]_{\mathrm{D}}{ }^{25}=-15.7^{\circ}(c 0.65$, $\left.\mathrm{CHCl}_{3}\right) ;{ }^{1} \mathrm{H} \mathrm{NMR}\left(600 \mathrm{MHz}, \mathrm{CDCl}_{3}\right) \delta 7.29(\mathrm{dd}, J=7.7,7.4 \mathrm{~Hz}, 2 \mathrm{H}), 7.20(\mathrm{~d}, J=7.1 \mathrm{~Hz}$, 2H), $7.19(\mathrm{dd}, J=7.3,6.4 \mathrm{~Hz}, 1 \mathrm{H}), 5.75-5.84(\mathrm{~m}, 2 \mathrm{H}), 5.21(\mathrm{~d}, J=10.3 \mathrm{~Hz}, 1 \mathrm{H}), 5.16$ $(\mathrm{d}, J=17.3 \mathrm{~Hz}, 1 \mathrm{H}), 5.14(\mathrm{~d}, J=10.2 \mathrm{~Hz}, 1 \mathrm{H}), 5.13(\mathrm{~d}, J=17.2 \mathrm{~Hz}, 1 \mathrm{H}), 3.57$ (ddd, $J=$ 9.2, 6.4, 2.9 Hz, 1H), $2.88-2.80(\mathrm{~m}, 2 \mathrm{H}), 2.66$ (ddd, $J=13.7,10.0,6.7 \mathrm{~Hz}, 1 \mathrm{H}), 1.86$ (dddd, $J=13.6,10.0,6.7,3.0 \mathrm{~Hz}, 1 \mathrm{H}), 1.72(\mathrm{br}, 1 \mathrm{H}), 1.65-1.71(\mathrm{~m}, 1 \mathrm{H}) .{ }^{13} \mathrm{C} \mathrm{NMR}(151$ $\left.\mathrm{MHz}_{\mathrm{CDCl}}\right) \delta 142.5,137.6,137.1,128.8,128.7,126.1,118.2,117.6,72.6,55.6,36.3$, 32.4. HRMS (ESI ${ }^{+}$): $m / z$ for $\mathrm{C}_{14} \mathrm{H}_{22} \mathrm{NO}\left[\mathrm{M}+\mathrm{NH}_{4}\right]^{+}$calcd. 220.1701 , found 220.1693 .

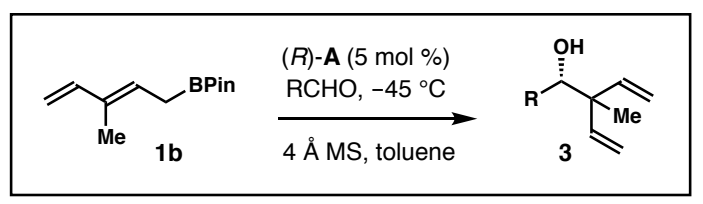

General procedure for the syntheses of homoallylic alcohols 3: To a reaction flask containing a stir bar and freshly activated $4 \AA$ MS (50 mg) was added phosphoric acid $(R)$-A $(3.8 \mathrm{mg}, 0.005 \mathrm{mmol})$. Toluene $(0.3 \mathrm{~mL})$ was added to the flask followed by drop wise addition of freshly distilled aldehyde $(0.1 \mathrm{mmol}$, if it is a liquid). The mixture was placed in a $-45{ }^{\circ} \mathrm{C}$ cold bath and stirred for $5 \mathrm{~min}$. Then allylboronate $\mathbf{1 b}(0.13 \mathrm{mmol})$ was added slowly to the reaction mixture via a microliter syringe. The mixture was kept at $-45{ }^{\circ} \mathrm{C}$ and stirred for $48 \mathrm{~h}$. After complete consumption of the aldehyde, sat. $\mathrm{NaHCO}_{3}$ $(1.0 \mathrm{~mL})$ was added to the reaction mixture followed by slow addition of $30 \% \mathrm{H}_{2} \mathrm{O}_{2}(0.5$ $\mathrm{mL})$ at $0{ }^{\circ} \mathrm{C}$. The reaction was stirred vigorously for $3 \mathrm{~h}$; then diethyl ether $(1 \mathrm{~mL})$ and brine $(1 \mathrm{~mL})$ was added. The organic layer was separated and the aqueous layer was extracted with $\mathrm{Et}_{2} \mathrm{O}(3 \times 2 \mathrm{~mL})$. The combined organic extracts were dried over anhydrous sodium sulfate, filtered, and concentrated under reduced pressure. Purification of the crude product was performed by flash chromatography (gradient elution with 
hexane and ethyl acetate) to give product 3.

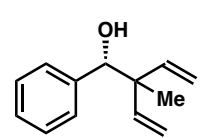

(S)-2-Methyl-1-phenyl-2-vinylbut-3-en-1-ol (3a) Prepared according to the general procedure. The crude mixture was purified by flash column chromatography (gradient elution with hexane and ethyl acetate, 20:1 to $10: 1)$ to give compound $\mathbf{3 a}$ as colorless oil in $84 \%$ yield $(15.8 \mathrm{mg})$. The enantiomeric excess was determined by HPLC analysis to be $93 \%$ ee $\left(254 \mathrm{~nm}, 25^{\circ} \mathrm{C}\right) ; \mathrm{t}_{1}=5.57 \mathrm{~min}, \mathrm{t}_{2}$ $=6.89 \mathrm{~min}\left[(\right.$ Chiralpak IC) hexane $/ i-\mathrm{PrOH}, 95: 5,1.0 \mathrm{~mL} / \mathrm{min}] ;[\alpha]_{\mathrm{D}}{ }^{25}=32.5^{\circ}$ (c 0.40, $\left.\mathrm{CHCl}_{3}\right) ;{ }^{1} \mathrm{H} \mathrm{NMR}\left(600 \mathrm{MHz}, \mathrm{CDCl}_{3}\right) \delta 7.26-7.32(\mathrm{~m}, 5 \mathrm{H}), 6.04(\mathrm{dd}, J=17.6,10.8 \mathrm{~Hz}$, $1 \mathrm{H}), 5.92(\mathrm{dd}, J=17.5,10.7 \mathrm{~Hz}, 1 \mathrm{H}), 5.22(\mathrm{~d}, J=10.8 \mathrm{~Hz}, 1 \mathrm{H}), 5.14(\mathrm{~d}, J=10.7 \mathrm{~Hz}, 1 \mathrm{H})$, $5.06(\mathrm{~d}, J=17.6 \mathrm{~Hz}, 1 \mathrm{H}), 5.05(\mathrm{~d}, J=17.5 \mathrm{~Hz}, 1 \mathrm{H}), 4.54$ (s, 1H), 2.12 (br, 1H), 1.07 (s, $3 \mathrm{H}) .{ }^{13} \mathrm{C}$ NMR $\left(151 \mathrm{MHz}, \mathrm{CDCl}_{3}\right) \delta 142.9,141.5,140.3,128.2,127.9,127.8,115.8$, 115.1, 80.0, 49.5, 18.9. HRMS $\left(\mathrm{ESI}^{+}\right): \mathrm{m} / z$ for $\mathrm{C}_{13} \mathrm{H}_{15}[\mathrm{M}-\mathrm{OH}]^{+}$calcd. 171.1174, found 171.1180 .

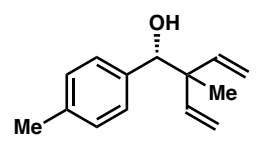

(S)-2-Methyl-1-(p-tolyl)-2-vinylbut-3-en-1-ol (3b) Prepared according to the general procedure. The crude mixture was purified by flash column chromatography (gradient elution with hexane and ethyl acetate, $20: 1$ to $10: 1)$ to give compound $\mathbf{3 b}$ as colorless oil in $90 \%$ yield (18.2 $\mathrm{mg}$ ). The enantiomeric excess was determined by HPLC analysis to be $97 \%$ ee $\left(254 \mathrm{~nm}, 25{ }^{\circ} \mathrm{C}\right) ; \mathrm{t}_{1}$ $=5.18 \mathrm{~min}, \mathrm{t}_{2}=5.51 \mathrm{~min}\left[(\right.$ Chiralpak IC) hexane $/ i-\mathrm{PrOH}, 95: 5,1.0 \mathrm{~mL} / \mathrm{min}] ;[\alpha]_{\mathrm{D}}{ }^{25}=$ $28.7^{\circ}\left(c 0.60, \mathrm{CHCl}_{3}\right) ;{ }^{1} \mathrm{H}$ NMR $\left(600 \mathrm{MHz}, \mathrm{CDCl}_{3}\right) \delta 7.17(\mathrm{~d}, J=7.8 \mathrm{~Hz}, 2 \mathrm{H}), 7.11(\mathrm{~d}, J$ $=7.8 \mathrm{~Hz}, 2 \mathrm{H}), 6.04(\mathrm{dd}, J=17.6,10.8 \mathrm{~Hz}, 1 \mathrm{H}), 5.92(\mathrm{dd}, J=17.5,10.7 \mathrm{~Hz}, 1 \mathrm{H}), 5.21$ (d, $J=10.8 \mathrm{~Hz}, 1 \mathrm{H}), 5.13(\mathrm{~d}, J=10.7 \mathrm{~Hz}, 1 \mathrm{H}), 5.09(\mathrm{~d}, J=17.6 \mathrm{~Hz}, 1 \mathrm{H}), 5.08(\mathrm{~d}, J=17.5$ $\mathrm{Hz}, 1 \mathrm{H}), 4.51(\mathrm{~d}, J=2.2 \mathrm{~Hz}, 1 \mathrm{H}), 2.33(\mathrm{~s}, 3 \mathrm{H}), 2.06(\mathrm{~d}, J=2.7 \mathrm{~Hz}, 1 \mathrm{H}), 1.06(\mathrm{~s}, 3 \mathrm{H}) .{ }^{13} \mathrm{C}$ NMR $\left(151 \mathrm{MHz}, \mathrm{CDCl}_{3}\right) \delta 143.0,141.7,137.5,137.4,128.5,128.1,115.7,115.0,79.9$, 49.5, 21.5, 19.0. HRMS (ESI $\left.{ }^{+}\right): m / z$ for $\mathrm{C}_{14} \mathrm{H}_{18} \mathrm{ONa}[\mathrm{M}+\mathrm{Na}]^{+}$calcd. 225.1255, found 225.1278 .

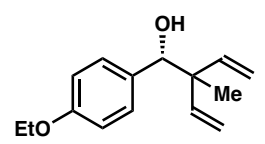

(S)-1-(4-Ethoxyphenyl)-2-methyl-2-vinylbut-3-en-1-ol (3c) Prepared according to the general procedure. The crude mixture was purified by flash column chromatography (gradient elution with hexane and ethyl acetate, 20:1 to 10:1) to give compound $\mathbf{3 c}$ as colorless oil in 93\% yield (21.6 $\mathrm{mg})$. The enantiomeric excess was determined by HPLC analysis to be $98 \%$ ee $\left(254 \mathrm{~nm}, 25{ }^{\circ} \mathrm{C}\right) ; \mathrm{t}_{1}$ $=7.97 \mathrm{~min}, \mathrm{t}_{2}=10.1 \mathrm{~min}\left[(\right.$ Chiralpak IB) hexane $/ i-\mathrm{PrOH}, 95: 5,1.0 \mathrm{~mL} / \mathrm{min}] ;[\alpha]_{\mathrm{D}}{ }^{25}=$ $44.3^{\circ}\left(c 0.80, \mathrm{CHCl}_{3}\right) ;{ }^{1} \mathrm{H} \mathrm{NMR}\left(600 \mathrm{MHz}, \mathrm{CDCl}_{3}\right) \delta 7.19(\mathrm{~d}, J=8.4 \mathrm{~Hz}, 2 \mathrm{H}), 6.82(\mathrm{~d}, J$ 
$=8.4 \mathrm{~Hz}, 2 \mathrm{H}), 6.04(\mathrm{dd}, J=17.6,10.8 \mathrm{~Hz}, 1 \mathrm{H}), 5.91(\mathrm{dd}, J=17.5,10.7 \mathrm{~Hz}, 1 \mathrm{H}), 5.21(\mathrm{~d}$, $J=10.8 \mathrm{~Hz}, 1 \mathrm{H}), 5.12(\mathrm{~d}, J=10.7 \mathrm{~Hz}, 1 \mathrm{H}), 5.05(\mathrm{~d}, J=17.6 \mathrm{~Hz}, 1 \mathrm{H}), 5.04$ (d, $J=17.5$ $\mathrm{Hz}, 1 \mathrm{H}), 4.49$ (d, $J=2.3 \mathrm{~Hz}, 1 \mathrm{H}), 4.02$ (q, $J=7.0 \mathrm{~Hz}, 2 \mathrm{H}), 2.06$ (d, $J=2.6 \mathrm{~Hz}, 1 \mathrm{H}), 1.41$ $(\mathrm{t}, J=7.0 \mathrm{~Hz}, 3 \mathrm{H}), 1.05(\mathrm{~s}, 3 \mathrm{H}) .{ }^{13} \mathrm{C} \mathrm{NMR}\left(151 \mathrm{MHz}, \mathrm{CDCl}_{3}\right) \delta 158.6,143.1,141.6$, $132.4,129.3,115.7,115.0,113.6,79.7,63.6,49.6,19.0,15.2$. HRMS $\left(\mathrm{ESI}^{+}\right): \mathrm{m} / z$ for $\mathrm{C}_{15} \mathrm{H}_{20} \mathrm{O}_{2} \mathrm{Na}[\mathrm{M}+\mathrm{Na}]^{+}$calcd. 255.1361, found 255.1379.

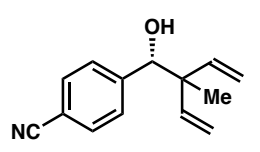

(S)-4-(1-Hydroxy-2-methyl-2-vinylbut-3-en-1-yl)benzonitrile

(3d)

Prepared according to the general procedure. The crude mixture was purified by flash column chromatography (gradient elution with hexane and ethyl acetate, 20:1 to 5:1) The crude mixture was purified by flash column chromatography to give compound $\mathbf{3 d}$ as colorless oil in 95\% yield $(20.3 \mathrm{mg})$. The enantiomeric excess was determined by HPLC analysis to be $99 \%$ ee $\left(254 \mathrm{~nm}, 25{ }^{\circ} \mathrm{C}\right) ; \mathrm{t}_{1}$ $=10.6 \mathrm{~min}, \mathrm{t}_{2}=11.3 \mathrm{~min}\left[(\right.$ Chiralpak IC) hexane $/ i-\mathrm{PrOH}, 95: 5,1.0 \mathrm{~mL} / \mathrm{min}] ;[\alpha]_{\mathrm{D}}{ }^{25}=$ $36.6^{\circ}$ (c 0.70, $\left.\mathrm{CHCl}_{3}\right) ;{ }^{1} \mathrm{H} \mathrm{NMR}\left(600 \mathrm{MHz}, \mathrm{CDCl}_{3}\right) \delta 7.59(\mathrm{~d}, J=8.1 \mathrm{~Hz}, 2 \mathrm{H}), 7.40(\mathrm{~d}, J$ $=8.1 \mathrm{~Hz}, 2 \mathrm{H}), 6.00(\mathrm{dd}, J=17.6,10.8 \mathrm{~Hz}, 1 \mathrm{H}), 5.85(\mathrm{dd}, J=17.5,10.7 \mathrm{~Hz}, 1 \mathrm{H}), 5.26(\mathrm{~d}$, $J=10.8 \mathrm{~Hz}, 1 \mathrm{H}), 5.17(\mathrm{~d}, J=10.7 \mathrm{~Hz}, 1 \mathrm{H}), 5.08(\mathrm{~d}, J=18.4 \mathrm{~Hz}, 1 \mathrm{H}), 5.05(\mathrm{~d}, J=17.7$ $\mathrm{Hz}, 1 \mathrm{H}), 4.59$ (d, $J=2.2 \mathrm{~Hz}, 1 \mathrm{H}), 2.26$ (d, $J=2.4 \mathrm{~Hz}, 1 \mathrm{H}), 1.04$ (s, 3H). ${ }^{13} \mathrm{C}$ NMR (151 $\left.\mathrm{MHz}_{\mathrm{CDCl}}\right) \delta 145.5,141.9,140.7,131.5,128.9,119.3,116.6,116.1,111.5,79.1,49.7$, 18.4. HRMS (ESI $\left.{ }^{+}\right): m / z$ for $\mathrm{C}_{14} \mathrm{H}_{16} \mathrm{ON}[\mathrm{M}+\mathrm{H}]^{+}$calcd. 214.1232, found 214.1243.

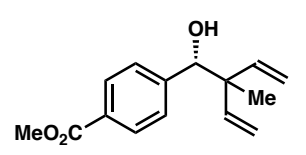

Methyl (S)-4-(1-hydroxy-2-methyl-2-vinylbut-3-en-1-yl)benzoate

(3e) Prepared according to the general procedure. The crude mixture was purified by flash column chromatography (gradient elution with hexane and ethyl acetate, 20:1 to 5:1) to give compound $\mathbf{3 e}$ as colorless oil in $96 \%$ yield (23.6 mg). The enantiomeric excess was determined by HPLC analysis to be $98 \%$ ee (254 $\left.\mathrm{nm}, 25{ }^{\circ} \mathrm{C}\right) ; \mathrm{t}_{1}=9.46 \mathrm{~min}, \mathrm{t}_{2}=10.9 \mathrm{~min}$ [(Chiralpak IC) hexane $/ i-\mathrm{PrOH}, 95: 5,1.0$ $\mathrm{mL} / \mathrm{min}] ;[\alpha]_{\mathrm{D}}{ }^{25}=33.9^{\circ}\left(c 0.85, \mathrm{CHCl}_{3}\right) ;{ }^{1} \mathrm{H} \mathrm{NMR}\left(600 \mathrm{MHz}, \mathrm{CDCl}_{3}\right) \delta 7.96(\mathrm{~d}, J=8.0$ $\mathrm{Hz}, 2 \mathrm{H}), 7.36$ (d, $J=8.0 \mathrm{~Hz}, 2 \mathrm{H}), 6.02$ (dd, $J=17.6,10.8 \mathrm{~Hz}, 1 \mathrm{H}), 5.88$ (dd, $J=17.5$, $10.7 \mathrm{~Hz}, 1 \mathrm{H}), 5.24(\mathrm{~d}, J=10.8 \mathrm{~Hz}, 1 \mathrm{H}), 5.15(\mathrm{~d}, J=10.7 \mathrm{~Hz}, 1 \mathrm{H}), 5.06(\mathrm{~d}, J=17.6 \mathrm{~Hz}$, $1 \mathrm{H}), 5.04(\mathrm{~d}, J=17.5 \mathrm{~Hz}, 1 \mathrm{H}), 4.60$ (d, $J=2.1 \mathrm{~Hz}, 1 \mathrm{H}), 3.91(\mathrm{~s}, 3 \mathrm{H}), 2.22$ (d, $J=2.3 \mathrm{~Hz}$, $1 \mathrm{H}), 1.05(\mathrm{~s}, 3 \mathrm{H}) .{ }^{13} \mathrm{C} \mathrm{NMR}\left(151 \mathrm{MHz}, \mathrm{CDCl}_{3}\right) \delta 167.4,145.4,142.3,141.1,129.6$, 129.0, 128.2, 116.2, 115.6, 79.4, 52.5, 49.6, 18.6. HRMS $\left(\mathrm{ESI}^{+}\right): \mathrm{m} / z$ for $\mathrm{C}_{15} \mathrm{H}_{19} \mathrm{O}_{3}$ [M$+\mathrm{H}]^{+}$calcd. 247.1334, found 247.1329. 


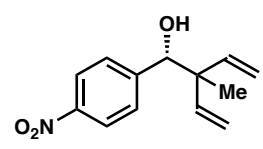

(S)-2-Methyl-1-(4-nitrophenyl)-2-vinylbut-3-en-1-ol (3f) Prepared according to the general procedure. The crude mixture was purified by flash column chromatography (gradient elution with hexane and ethyl acetate, 20:1 to 5:1) to give compound $\mathbf{3 f}$ as colorless oil in $97 \%$ yield $(22.6 \mathrm{mg})$. The enantiomeric excess was determined by HPLC analysis to be $98 \%$ ee $\left(254 \mathrm{~nm}, 25^{\circ} \mathrm{C}\right)$; $\mathrm{t}_{1}$ $=8.68 \mathrm{~min}, \mathrm{t}_{2}=9.37 \mathrm{~min}$ [(Chiralpak IE) hexane $\left./ i-\mathrm{PrOH}, 95: 5,1.0 \mathrm{~mL} / \mathrm{min}\right] ;[\alpha]_{\mathrm{D}}{ }^{25}=$ $25.3^{\circ}$ (c 0.80, $\left.\mathrm{CHCl}_{3}\right) ;{ }^{1} \mathrm{H} \mathrm{NMR}\left(600 \mathrm{MHz}, \mathrm{CDCl}_{3}\right) \delta 8.16(\mathrm{~d}, J=8.3 \mathrm{~Hz}, 2 \mathrm{H}), 7.46(\mathrm{~d}, J$ $=8.4 \mathrm{~Hz}, 2 \mathrm{H}), 6.02(\mathrm{dd}, J=17.6,10.8 \mathrm{~Hz}, 1 \mathrm{H}), 5.86(\mathrm{dd}, J=17.5,10.7 \mathrm{~Hz}, 1 \mathrm{H}), 5.27(\mathrm{~d}$, $J=10.8 \mathrm{~Hz}, 1 \mathrm{H}), 5.18(\mathrm{~d}, J=10.7 \mathrm{~Hz}, 1 \mathrm{H}), 5.08(\mathrm{~d}, J=18.6 \mathrm{~Hz}, 1 \mathrm{H}), 5.06(\mathrm{~d}, J=18.6$ $\mathrm{Hz}, 1 \mathrm{H}), 4.65(\mathrm{~s}, 1 \mathrm{H}), 2.29(\mathrm{~d}, J=1.7 \mathrm{~Hz}, 1 \mathrm{H}), 1.05(\mathrm{~s}, 3 \mathrm{H}) .{ }^{13} \mathrm{C} \mathrm{NMR}\left(151 \mathrm{MHz}, \mathrm{CDCl}_{3}\right)$ $\delta$ 147.6, 147.5, 141.8, 140.6, 129.0, 122.9, 116.8, 116.2, 78.9, 49.7, 18.3. HRMS $\left(\mathrm{ESI}^{+}\right)$: $m / z$ for $\mathrm{C}_{13} \mathrm{H}_{15} \mathrm{NO}_{3} \mathrm{Na}[\mathrm{M}+\mathrm{Na}]^{+}$calcd. 256.0950, found 256.0972.

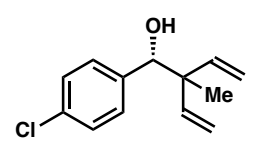

(S)-1-(4-Chlorophenyl)-2-methyl-2-vinylbut-3-en-1-ol (3g) Prepared according to the general procedure. The crude mixture was purified by flash column chromatography (gradient elution with hexane and ethyl acetate, $20: 1$ to $10: 1$ ) to give compound $\mathbf{3 g}$ as colorless oil in $93 \%$ yield $(20.7 \mathrm{mg})$. The enantiomeric excess was determined by HPLC analysis to be $97 \%$ ee $\left(254 \mathrm{~nm}, 25^{\circ} \mathrm{C}\right) ; \mathrm{t}_{1}$ $=4.29 \mathrm{~min}, \mathrm{t}_{2}=4.68 \mathrm{~min}$ [(Chiralpak IB) hexane $\left./ i-\mathrm{PrOH}, 95: 5,1.0 \mathrm{~mL} / \mathrm{min}\right] ;[\alpha]_{\mathrm{D}}{ }^{25}=$ $46.3^{\circ}$ (c 0.73, $\left.\mathrm{CHCl}_{3}\right) ;{ }^{1} \mathrm{H}$ NMR (600 MHz, $\left.\mathrm{CDCl}_{3}\right) \delta 7.27$ (d, $\left.J=8.2 \mathrm{~Hz}, 2 \mathrm{H}\right), 7.22(\mathrm{~d}, J$ $=8.4 \mathrm{~Hz}, 2 \mathrm{H}), 6.01(\mathrm{dd}, J=17.6,10.8 \mathrm{~Hz}, 1 \mathrm{H}), 5.87(\mathrm{dd}, J=17.5,10.7 \mathrm{~Hz}, 1 \mathrm{H}), 5.24(\mathrm{~d}$, $J=10.8 \mathrm{~Hz}, 1 \mathrm{H}), 5.14(\mathrm{~d}, J=10.7 \mathrm{~Hz}, 1 \mathrm{H}), 5.07$ (d, $J=17.6 \mathrm{~Hz}, 1 \mathrm{H}), 5.04$ (d, $J=17.5$ $\mathrm{Hz}, 1 \mathrm{H}), 4.52(\mathrm{~d}, J=2.3 \mathrm{~Hz}, 1 \mathrm{H}), 2.14(\mathrm{~d}, J=2.6 \mathrm{~Hz}, 1 \mathrm{H}), 1.04(\mathrm{~s}, 3 \mathrm{H}) .{ }^{13} \mathrm{C}$ NMR $(151$ $\left.\mathrm{MHz}, \mathrm{CDCl}_{3}\right) \delta 142.5,141.1,138.7,133.5,129.5,127.9,116.2,115.5,79.2,49.5,18.6$. HRMS (ESI ${ }^{+}$): $m / z$ for $\mathrm{C}_{13} \mathrm{H}_{15} \mathrm{OClNa}[\mathrm{M}+\mathrm{Na}]^{+}$calcd. 245.0709, found 245.0744 .

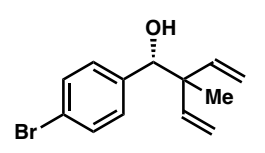

(S)-1-(4-Bromophenyl)-2-methyl-2-vinylbut-3-en-1-ol (3h) Prepared according to the general procedure. The crude mixture was purified by flash column chromatography (gradient elution with hexane and ethyl acetate, 20:1 to 10:1) to give compound $\mathbf{3 h}$ as colorless oil in $95 \%$ yield $(25.4 \mathrm{mg})$. The enantiomeric excess was determined by HPLC analysis to be $98 \%$ ee $\left(254 \mathrm{~nm}, 25^{\circ} \mathrm{C}\right)$; $\mathrm{t}_{1}$ $=4.47 \mathrm{~min}, \mathrm{t}_{2}=5.06 \mathrm{~min}$ [(Chiralpak IB) hexane $\left./ i-\mathrm{PrOH}, 95: 5,1.0 \mathrm{~mL} / \mathrm{min}\right] ;[\alpha]_{\mathrm{D}}{ }^{25}=$ $38.4^{\circ}\left(c 0.97, \mathrm{CHCl}_{3}\right) ;{ }^{1} \mathrm{H} \mathrm{NMR}\left(600 \mathrm{MHz}, \mathrm{CDCl}_{3}\right) \delta 7.42(\mathrm{~d}, J=8.2 \mathrm{~Hz}, 2 \mathrm{H}), 7.16(\mathrm{~d}, J$ $=8.2 \mathrm{~Hz}, 2 \mathrm{H}), 6.01(\mathrm{dd}, J=17.6,10.8 \mathrm{~Hz}, 1 \mathrm{H}), 5.87(\mathrm{dd}, J=17.5,10.7 \mathrm{~Hz}, 1 \mathrm{H}), 5.24(\mathrm{~d}$, $J=10.8 \mathrm{~Hz}, 1 \mathrm{H}), 5.14(\mathrm{~d}, J=10.7 \mathrm{~Hz}, 1 \mathrm{H}), 5.07$ (d, $J=17.6 \mathrm{~Hz}, 1 \mathrm{H}), 5.05(\mathrm{~d}, J=17.5$ $\mathrm{Hz}, 1 \mathrm{H}), 4.52(\mathrm{~d}, J=1.7 \mathrm{~Hz}, 1 \mathrm{H}), 2.15(\mathrm{~d}, J=2.0 \mathrm{~Hz}, 1 \mathrm{H}), 1.04(\mathrm{~s}, 3 \mathrm{H}) .{ }^{13} \mathrm{C}$ NMR $(151$ 
$\left.\mathrm{MHz}, \mathrm{CDCl}_{3}\right) \delta 142.5,141.1,139.2,130.8,129.9,121.7,116.2,115.5,79.3,49.5,18.6$. HRMS (ESI ${ }^{+}$): $m / z$ for $\mathrm{C}_{13} \mathrm{H}_{14} \mathrm{Br}[\mathrm{M}-\mathrm{OH}]^{+}$calcd. 249.0279, found 249.0287 .

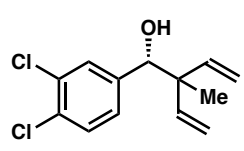

(S)-1-(3,4-Dichlorophenyl)-2-methyl-2-vinylbut-3-en-1-ol

Prepared according to the general procedure. The crude mixture was purified by flash column chromatography (gradient elution with hexane and ethyl acetate, $20: 1$ to $10: 1)$ to give compound $\mathbf{3 i}$ as colorless oil in $91 \%$ yield (23.4 $\mathrm{mg})$. The enantiomeric excess was determined by HPLC analysis to be $97 \%$ ee $(254 \mathrm{~nm}$, $\left.25{ }^{\circ} \mathrm{C}\right) ; \mathrm{t}_{1}=5.53 \mathrm{~min}, \mathrm{t}_{2}=5.93 \mathrm{~min}$ [(Chiralpak IB) hexane $/ i-\mathrm{PrOH}, 95: 5,1.0 \mathrm{~mL} / \mathrm{min}$ ]; $[\alpha]_{\mathrm{D}}{ }^{25}=30.0^{\circ}\left(c 0.85, \mathrm{CHCl}_{3}\right) ;{ }^{1} \mathrm{H} \mathrm{NMR}\left(600 \mathrm{MHz}, \mathrm{CDCl}_{3}\right) \delta 7.39(\mathrm{~d}, J=1.6 \mathrm{~Hz}, 1 \mathrm{H})$, $7.36(\mathrm{~d}, J=8.3 \mathrm{~Hz}, 1 \mathrm{H}), 7.11(\mathrm{dd}, J=8.3,1.7 \mathrm{~Hz}, 1 \mathrm{H}), 5.99(\mathrm{dd}, J=17.6,10.8 \mathrm{~Hz}, 1 \mathrm{H})$, $5.85(\mathrm{dd}, J=17.5,10.7 \mathrm{~Hz}, 1 \mathrm{H}), 5.26$ (d, $J=10.8 \mathrm{~Hz}, 1 \mathrm{H}), 5.17$ (d, $J=10.7 \mathrm{~Hz}, 1 \mathrm{H})$, $5.08(\mathrm{~d}, J=16.7 \mathrm{~Hz}, 1 \mathrm{H}), 5.05(\mathrm{~d}, J=17.0 \mathrm{~Hz}, 1 \mathrm{H}), 4.49$ (d, $J=2.4 \mathrm{~Hz}, 1 \mathrm{H}), 2.18$ (d, $J=$ $2.6 \mathrm{~Hz}, 1 \mathrm{H}), 1.04(\mathrm{~s}, 3 \mathrm{H}) .{ }^{13} \mathrm{C} \mathrm{NMR}\left(151 \mathrm{MHz}, \mathrm{CDCl}_{3}\right) \delta$ 142.1, 140.8, 140.4, 131.9, 131.7, 130.0, 129.6, 127.6, 116.5, 115.9, 78.6, 49.6, 18.5. HRMS $\left(\mathrm{ESI}^{+}\right): \mathrm{m} / \mathrm{z}$ for $\mathrm{C}_{13} \mathrm{H}_{13} \mathrm{Cl}_{2}[\mathrm{M}-\mathrm{OH}]^{+}$calcd. 239.0394 found 239.0410.

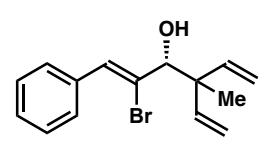

$(R, Z)-2-B r o m o-4-m e t h y l-1-p h e n y l-4-v i n y l h e x a-1,5-d i e n-3-o l$

Prepared according to the general procedure. The crude mixture was purified by flash column chromatography (gradient elution with hexane and ethyl acetate, $20: 1$ to $10: 1)$ to give compound $\mathbf{3} \mathbf{j}$ as colorless oil in $71 \%$ yield (20.8 $\mathrm{mg})$. The enantiomeric excess was determined by HPLC analysis to be $99 \%$ ee $(254 \mathrm{~nm}$, $\left.25{ }^{\circ} \mathrm{C}\right) ; \mathrm{t}_{1}=8.24 \mathrm{~min}, \mathrm{t}_{2}=9.32 \mathrm{~min}$ [(Chiralpak IC) hexane $/ i-\mathrm{PrOH}, 97: 3,1.0 \mathrm{~mL} / \mathrm{min}$ ]; $[\alpha]_{\mathrm{D}}{ }^{25}=-20.9^{\circ}\left(c 0.70, \mathrm{CHCl}_{3}\right) ;{ }^{1} \mathrm{H} \mathrm{NMR}\left(600 \mathrm{MHz}, \mathrm{CDCl}_{3}\right) \delta 7.58(\mathrm{~d}, J=7.7 \mathrm{~Hz}, 2 \mathrm{H})$, 7.37 (dd, $J=7.6,7.5 \mathrm{~Hz}, 2 \mathrm{H}), 7.31$ (dd, $J=7.4,7.1 \mathrm{~Hz}, 1 \mathrm{H}), 7.00$ (s, 1H), 6.13 (dd, $J=$ 17.5, $10.8 \mathrm{~Hz}, 1 \mathrm{H}), 6.09$ (dd, $J=17.5,10.8 \mathrm{~Hz}, 1 \mathrm{H}), 5.22$ (d, $J=10.7 \mathrm{~Hz}, 2 \mathrm{H}), 5.16$ (d, $J$ $=17.5 \mathrm{~Hz}, 1 \mathrm{H}), 5.15(\mathrm{~d}, J=17.5 \mathrm{~Hz}, 1 \mathrm{H}), 4.23(\mathrm{~d}, J=7.1 \mathrm{~Hz}, 1 \mathrm{H}), 2.37(\mathrm{~d}, J=7.2 \mathrm{~Hz}$, $1 \mathrm{H}), 1.28(\mathrm{~s}, 3 \mathrm{H}) .{ }^{13} \mathrm{C} \mathrm{NMR}\left(151 \mathrm{MHz}, \mathrm{CDCl}_{3}\right) \delta 141.9,141.7,135.5,131.5,129.5$, 128.5 (2 overlapping ${ }^{13} \mathrm{C}$ signals), 126.3, 115.6, 115.4, 82.5, 49.6, 20.8. HRMS $\left(\mathrm{ESI}^{+}\right)$: $m / z$ for $\mathrm{C}_{15} \mathrm{H}_{17} \mathrm{ONaBr}[\mathrm{M}+\mathrm{Na}]^{+}$calcd. 315.0360, found 315.0373 .

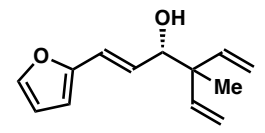

(S,E)-1-(Furan-2-yl)-4-methyl-4-vinylhexa-1,5-dien-3-ol

Prepared according to the general procedure. The crude mixture was purified by flash column chromatography (gradient elution with hexane and ethyl acetate, $20: 1$ to $10: 1)$ to give compound $3 \mathbf{k}$ as colorless oil in $88 \%$ yield (18.0 $\mathrm{mg})$. The enantiomeric excess was determined by HPLC analysis to be $97 \%$ ee $(254 \mathrm{~nm}$, 
$\left.25{ }^{\circ} \mathrm{C}\right) ; \mathrm{t}_{1}=7.86 \mathrm{~min}, \mathrm{t}_{2}=9.62 \mathrm{~min}$ [(Chiralpak IA) hexane $/ i-\mathrm{PrOH}, 95: 5,1.0 \mathrm{~mL} / \mathrm{min}$; $[\alpha]_{\mathrm{D}}^{25}=22.7^{\circ}\left(c 0.60, \mathrm{CHCl}_{3}\right) ;{ }^{1} \mathrm{H} \mathrm{NMR}\left(600 \mathrm{MHz}, \mathrm{CDCl}_{3}\right) \delta 7.34(\mathrm{~s}, 1 \mathrm{H}), 6.43(\mathrm{~d}, J=$ $15.8 \mathrm{~Hz}, 1 \mathrm{H}), 6.36(\mathrm{dd}, J=3.1,1.7 \mathrm{~Hz}, 1 \mathrm{H}), 6.23(\mathrm{~d}, J=3.2 \mathrm{~Hz}, 1 \mathrm{H}), 6.18$ (dd, $J=15.8$, $6.2 \mathrm{~Hz}, 1 \mathrm{H}), 6.00(\mathrm{dd}, J=17.6,10.8 \mathrm{~Hz}, 1 \mathrm{H}), 5.94(\mathrm{dd}, J=17.5,10.8 \mathrm{~Hz}, 1 \mathrm{H}), 5.23(\mathrm{~d}, J$ $=10.8 \mathrm{~Hz}, 1 \mathrm{H}), 5.20(\mathrm{~d}, J=10.8 \mathrm{~Hz}, 1 \mathrm{H}), 5.14(\mathrm{~d}, J=17.6 \mathrm{~Hz}, 2 \mathrm{H}), 4.08-4.07(\mathrm{~m}, 1 \mathrm{H})$, $1.77(\mathrm{~d}, J=4.3 \mathrm{~Hz}, 1 \mathrm{H}), 1.16$ (s, 3H). ${ }^{13} \mathrm{C}$ NMR $\left(151 \mathrm{MHz}, \mathrm{CDCl}_{3}\right) \delta 152.7,142.3,142.2$, 141.9, 127.0, 120.6, 115.7, 115.6, 111.6, 108.4, 77.8, 49.2, 19.1. HRMS $\left(\mathrm{ESI}^{+}\right): \mathrm{m} / \mathrm{z}$ for $\mathrm{C}_{13} \mathrm{H}_{16} \mathrm{O}_{2} \mathrm{Na}[\mathrm{M}+\mathrm{Na}]^{+}$calcd. 227.1048, found 227.1061.

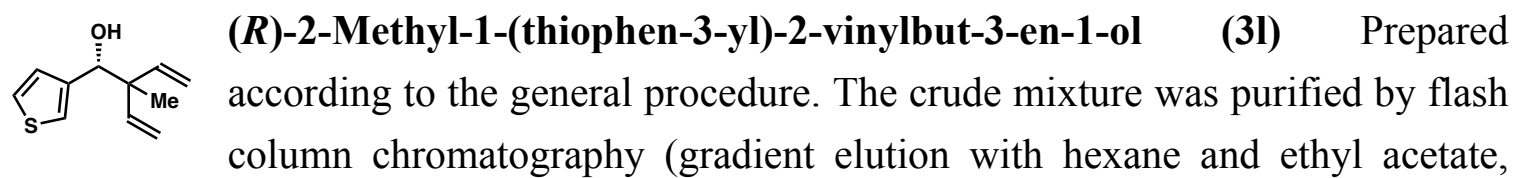

$20: 1$ to $10: 1)$ to give compound 31 as colorless oil in $96 \%$ yield $(18.7 \mathrm{mg})$. The enantiomeric excess was determined by HPLC analysis to be $95 \%$ ee $\left(254 \mathrm{~nm}, 25^{\circ} \mathrm{C}\right) ; \mathrm{t}_{1}$ $=5.75 \mathrm{~min}, \mathrm{t}_{2}=7.82 \mathrm{~min}$ [(Chiralpak IC) hexane $\left./ i-\operatorname{PrOH}, 95: 5,1.0 \mathrm{~mL} / \mathrm{min}\right] ;[\alpha]_{\mathrm{D}}{ }^{25}=$ $52.7^{\circ}\left(c 0.60, \mathrm{CHCl}_{3}\right) ;{ }^{1} \mathrm{H} \mathrm{NMR}\left(600 \mathrm{MHz}, \mathrm{CDCl}_{3}\right) \delta 7.23(\mathrm{dd}, J=4.7,2.9 \mathrm{~Hz}, 1 \mathrm{H}), 7.16$ (app. s, 1H), 7.03 (d, $J=4.8 \mathrm{~Hz}, 1 \mathrm{H}), 6.04$ (dd, $J=17.6,10.8 \mathrm{~Hz}, 1 \mathrm{H}), 5.93$ (dd, $J=17.5$, $10.7 \mathrm{~Hz}, 1 \mathrm{H}), 5.23(\mathrm{~d}, J=10.8 \mathrm{~Hz}, 1 \mathrm{H}), 5.15(\mathrm{~d}, J=10.7 \mathrm{~Hz}, 1 \mathrm{H}), 5.10(\mathrm{~d}, J=17.6,1 \mathrm{H})$, $5.08(\mathrm{~d}, J=17.6,1 \mathrm{H}), 4.65(\mathrm{~d}, J=2.6 \mathrm{~Hz}, 1 \mathrm{H}), 2.09(\mathrm{~d}, J=3.0 \mathrm{~Hz}, 1 \mathrm{H}), 1.09(\mathrm{~s}, 3 \mathrm{H}) .{ }^{13} \mathrm{C}$ NMR (151 MHz, $\left.\mathrm{CDCl}_{3}\right) \delta 142.7,142.1,141.6,127.7,124.8,122.7,115.8,115.3,76.6$, 49.4, 19.0. HRMS (ESI $\left.{ }^{+}\right): m / z$ for $\mathrm{C}_{11} \mathrm{H}_{13} \mathrm{~S}[\mathrm{M}-\mathrm{OH}]^{+}$calcd. 177.0738, found 177.0745.

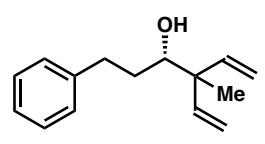

(S)-4-Methyl-1-phenyl-4-vinylhex-5-en-3-ol (3m) Prepared according to the general procedure. The crude mixture was purified by flash column chromatography (gradient elution with hexane and ethyl acetate, $20: 1$ to $10: 1)$ to give compound $\mathbf{3 m}$ as colorless oil in $84 \%$ yield $(18.2 \mathrm{mg})$. The enantiomeric excess was determined by HPLC analysis to be $94 \%$ ee $\left(254 \mathrm{~nm}, 25^{\circ} \mathrm{C}\right)$; $\mathrm{t}_{1}$ $=5.43 \mathrm{~min}, \mathrm{t}_{2}=8.09 \mathrm{~min}$ [(Chiralpak IC) hexane $\left./ i-\mathrm{PrOH}, 97: 3,1.0 \mathrm{~mL} / \mathrm{min}\right] ;[\alpha]_{\mathrm{D}}{ }^{25}=$ $-22.5^{\circ}$ (c 0.55, $\left.\mathrm{CHCl}_{3}\right) ;{ }^{1} \mathrm{H}$ NMR (400 MHz, $\left.\mathrm{CDCl}_{3}\right) \delta 7.26-7.30(\mathrm{~m}, 2 \mathrm{H}), 7.21-7.16$ (m, 3H), 5.90 (dd, $J=17.5,10.7 \mathrm{~Hz} 1 \mathrm{H}), 5.84(\mathrm{dd}, J=16.9,10.1 \mathrm{~Hz}, 1 \mathrm{H}), 5.17$ (d, $J=$ $10.6 \mathrm{~Hz}, 1 \mathrm{H}), 5.15$ (dd, $J=10.6,1.1 \mathrm{~Hz}, 1 \mathrm{H}), 5.08$ (d, $J=17.6 \mathrm{~Hz}, 2 \mathrm{H}), 3.40$ (d, $J=9.9$ Hz, 1H), 2.92 (ddd, $J=14.4,10.2,4.9 \mathrm{~Hz}, 1 \mathrm{H}), 2.61$ (ddd, $J=13.7,9.6,7.1 \mathrm{~Hz}, 1 \mathrm{H}$ ), $1.79-1.87(\mathrm{~m}, 1 \mathrm{H}), 1.54-1.62(\mathrm{~m}, 2 \mathrm{H}), 1.11(\mathrm{~s}, 3 \mathrm{H}) .{ }^{13} \mathrm{C} \mathrm{NMR}\left(101 \mathrm{MHz}, \mathrm{CDCl}_{3}\right) \delta$ $142.59,142.58,142.5,128.8,128.7,126.1,115.3,115.0,76.5,49.0,33.7,33.5,18.1$. HRMS (ESI $\left.{ }^{+}\right): m / z$ for $\mathrm{C}_{15} \mathrm{H}_{19}[\mathrm{M}-\mathrm{OH}]^{+}$calcd. 199.1487, found 199.1504. 


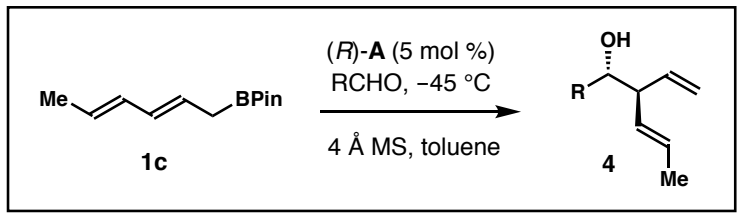

General procedure for the syntheses of homoallylic alcohols 4: To a reaction flask containing a stir bar and freshly activated $4 \AA \mathrm{MS}(50 \mathrm{mg})$ was added phosphoric acid $(R)$-A $(3.8 \mathrm{mg}, 0.005 \mathrm{mmol})$. Toluene $(0.3 \mathrm{~mL})$ was added to the flask followed by drop wise addition of freshly distilled aldehyde $(0.1 \mathrm{mmol}$, if it is a liquid $)$. The mixture was placed in a $-45{ }^{\circ} \mathrm{C}$ cold bath and stirred for $5 \mathrm{~min}$. Then allylboronate $1 \mathrm{c}(0.13 \mathrm{mmol})$ was added slowly to the reaction mixture via a microliter syringe. The mixture was kept at $-45{ }^{\circ} \mathrm{C}$ and stirred for $48 \mathrm{~h}$. After complete consumption of the aldehyde, sat. $\mathrm{NaHCO}_{3}$ $(1.0 \mathrm{~mL})$ was added to the reaction mixture followed by slow addition of $30 \% \mathrm{H}_{2} \mathrm{O}_{2}(0.5$ $\mathrm{mL})$ at $0{ }^{\circ} \mathrm{C}$. The reaction was stirred vigorously for $3 \mathrm{~h}$; then diethyl ether $(1 \mathrm{~mL})$ and brine $(1 \mathrm{~mL})$ was added. The organic layer was separated and the aqueous layer was extracted with $\mathrm{Et}_{2} \mathrm{O}(3 \times 2 \mathrm{~mL})$. The combined organic extracts were dried over anhydrous sodium sulfate, filtered, and concentrated under reduced pressure. Purification of the crude product was performed by flash chromatography (gradient elution with hexane and ethyl acetate) to give product 4.

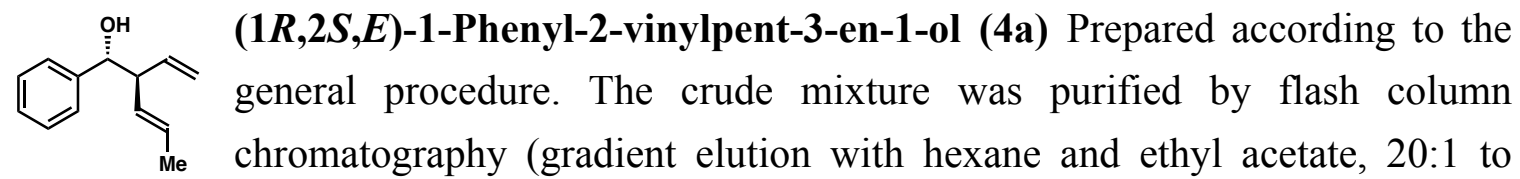
$10: 1)$ to give compound $\mathbf{4 a}$ as colorless oil in $93 \%$ yield $(17.5 \mathrm{mg})$. The enantiomeric excess was determined by HPLC analysis to be $95 \%$ ee $\left(254 \mathrm{~nm}, 25{ }^{\circ} \mathrm{C}\right) ; \mathrm{t}_{1}=5.16 \mathrm{~min}, \mathrm{t}_{2}$ $=5.58 \mathrm{~min}\left[(\right.$ Chiralpak IB) hexane $/ i-\mathrm{PrOH}, 95: 5,1.0 \mathrm{~mL} / \mathrm{min}] ;[\alpha]_{\mathrm{D}}{ }^{25}=55.5^{\circ}(c 0.58$, $\left.\mathrm{CHCl}_{3}\right) ;{ }^{1} \mathrm{H} \mathrm{NMR}\left(600 \mathrm{MHz}, \mathrm{CDCl}_{3}\right) \delta 7.36-7.29(\mathrm{~m}, 4 \mathrm{H}), 7.26-7.28(\mathrm{~m}, 1 \mathrm{H}), 5.81$ (ddd, $J=17.6,10.2,8.4 \mathrm{~Hz}, 1 \mathrm{H}), 5.41$ (dq, $J=15.4,6.4 \mathrm{~Hz}, 1 \mathrm{H}), 5.29$ (dd, $J=15.5,7.3$ $\mathrm{Hz}, 1 \mathrm{H}), 5.20$ (d, $J=10.2 \mathrm{~Hz}, 1 \mathrm{H}), 5.15(\mathrm{~d}, J=17.2 \mathrm{~Hz}, 1 \mathrm{H}), 4.55(\mathrm{~d}, J=7.0 \mathrm{~Hz}, 1 \mathrm{H})$, 3.04 (app. q, $J=7.5 \mathrm{~Hz}, 1 \mathrm{H}), 2.20$ (br, $1 \mathrm{H}), 1.61$ (d, $J=6.4 \mathrm{~Hz}, 3 \mathrm{H}) .{ }^{13} \mathrm{C}$ NMR $(151$ $\left.\mathrm{MHz}, \mathrm{CDCl}_{3}\right) \delta 142.2,137.8,129.4,128.41,128.36,127.8,127.2,118.2,76.6,55.6,18.5$. HRMS (ESI ${ }^{+}$): $m / z$ for $\mathrm{C}_{13} \mathrm{H}_{15}[\mathrm{M}-\mathrm{OH}]^{+}$calcd. 171.1174, found 171.1177.

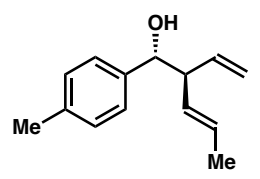

$(1 R, 2 S, E)-1-(p$-Tolyl)-2-vinylpent-3-en-1-ol (4b) Prepared according to the general procedure. The crude mixture was purified by flash column chromatography (gradient elution with hexane and ethyl acetate, 20:1 to 
10:1) to give compound $\mathbf{4 b}$ as colorless oil in $94 \%$ yield $(19.0 \mathrm{mg})$. The enantiomeric excess was determined by HPLC analysis to be $98 \%$ ee $\left(254 \mathrm{~nm}, 25^{\circ} \mathrm{C}\right) ; \mathrm{t}_{1}=6.13 \mathrm{~min}, \mathrm{t}_{2}$ $=6.90 \mathrm{~min}\left[(\right.$ Chiralpak IB) hexane $/ i-\mathrm{PrOH}, 95: 5,1.0 \mathrm{~mL} / \mathrm{min}] ;[\alpha]_{\mathrm{D}}{ }^{25}=45.8^{\circ}(c 0.65$, $\left.\mathrm{CHCl}_{3}\right) ;{ }^{1} \mathrm{H} \mathrm{NMR}\left(600 \mathrm{MHz}, \mathrm{CDCl}_{3}\right) \delta 7.19(\mathrm{~d}, J=7.7 \mathrm{~Hz}, 2 \mathrm{H}), 7.14(\mathrm{~d}, J=7.8 \mathrm{~Hz}, 2 \mathrm{H})$, $5.82(\mathrm{ddd}, J=17.8,10.2,7.7 \mathrm{~Hz}, 1 \mathrm{H}), 5.42(\mathrm{dq}, J=15.4,6.4 \mathrm{~Hz}, 1 \mathrm{H}), 5.29$ (dd, $J=15.4$, $7.2 \mathrm{~Hz}, 1 \mathrm{H}), 5.19$ (d, $J=10.2 \mathrm{~Hz}, 1 \mathrm{H}), 5.15$ (d, $J=17.2 \mathrm{~Hz}, 1 \mathrm{H}), 4.52$ (dd, $J=7.0,3.1$ $\mathrm{Hz}, 1 \mathrm{H}), 3.04$ (app. q, $J=7.4 \mathrm{~Hz}, 1 \mathrm{H}), 2.34$ (s, 3H), 2.15 (d, $J=3.2 \mathrm{~Hz}, 1 \mathrm{H}), 1.61$ (d, $J=$ $6.4 \mathrm{~Hz}, 3 \mathrm{H}) .{ }^{13} \mathrm{C} \mathrm{NMR}\left(151 \mathrm{MHz}, \mathrm{CDCl}_{3}\right) \delta 139.2,138.0,137.4,129.5,129.1,128.3$, 127.1, 118.1, 76.5, 55.5, 21.5, 18.5. HRMS (ESI $\left.{ }^{+}\right): m / z$ for $\mathrm{C}_{14} \mathrm{H}_{18} \mathrm{ONa}[\mathrm{M}+\mathrm{Na}]^{+}$calcd. 225.1255 , found 225.1270 .

$(1 R, 2 S, E)-1-(4-E t h o x y p h e n y l)-2-v i n y l p e n t-3-e n-1-o l ~(4 c)$ Prepared
according to the general procedure. The crude mixture was purified by
flash column chromatography (gradient elution with hexane and ethyl acetate, $20: 1$ to $10: 1)$ to give compound $\mathbf{4 c}$ as colorless oil in $85 \%$ yield (19.8 $\mathrm{mg})$. The enantiomeric excess was determined by HPLC analysis to be $98 \%$ ee $\left(254 \mathrm{~nm}, 25{ }^{\circ} \mathrm{C}\right) ; \mathrm{t}_{1}$ $=9.53 \mathrm{~min}, \mathrm{t}_{2}=12.7 \mathrm{~min}\left[(\right.$ Chiralpak IB) hexane $/ i-\mathrm{PrOH}, 95: 5,1.0 \mathrm{~mL} / \mathrm{min}] ;[\alpha]_{\mathrm{D}}{ }^{25}=$ $31.4^{\circ}\left(c 0.70, \mathrm{CHCl}_{3}\right) ;{ }^{1} \mathrm{H} \mathrm{NMR}\left(600 \mathrm{MHz}, \mathrm{CDCl}_{3}\right) \delta 7.21(\mathrm{~d}, J=8.5 \mathrm{~Hz}, 2 \mathrm{H}), 6.85(\mathrm{~d}, J$ $=8.5 \mathrm{~Hz}, 2 \mathrm{H}), 5.81(\mathrm{ddd}, J=17.5,9.9,8.6 \mathrm{~Hz}, 1 \mathrm{H}), 5.39$ (dq, $J=15.5,6.4 \mathrm{~Hz}, 1 \mathrm{H}), 5.26$ $(\mathrm{dd}, J=15.4,7.2 \mathrm{~Hz}, 1 \mathrm{H}), 5.20(\mathrm{~d}, J=10.2 \mathrm{~Hz}, 1 \mathrm{H}), 5.16(\mathrm{~d}, J=17.4 \mathrm{~Hz}, 1 \mathrm{H}), 4.48$ (dd, $J=7.3,2.8 \mathrm{~Hz}, 1 \mathrm{H}), 4.02$ (q, $J=7.0 \mathrm{~Hz}, 2 \mathrm{H}), 3.01$ (app. q, $J=7.6 \mathrm{~Hz}, 1 \mathrm{H}), 2.15$ (d, $J=$ $2.8 \mathrm{~Hz}, 1 \mathrm{H}), 1.60(\mathrm{~d}, J=6.4 \mathrm{~Hz}, 3 \mathrm{H}), 1.41(\mathrm{t}, J=7.0 \mathrm{~Hz}, 3 \mathrm{H}) .{ }^{13} \mathrm{C}$ NMR $(151 \mathrm{MHz}$, $\left.\mathrm{CDCl}_{3}\right) \delta 158.6,138.2,134.2,129.5,128.3,128.2,118.1,114.2,76.3,63.7,55.7,18.5$, 15.2. HRMS (ESI $\left.{ }^{+}\right): m / z$ for $\mathrm{C}_{15} \mathrm{H}_{20} \mathrm{O}_{2} \mathrm{Na}[\mathrm{M}+\mathrm{Na}]^{+}$calcd. 255.1361, found 255.1359.

Prepared according to the general procedure. The crude mixture was
purified by flash column chromatography (gradient elution with hexane and ethyl acetate, $20: 1$ to $5: 1)$ to give compound $\mathbf{4 d}$ as colorless oil in $88 \%$ yield (18.8 $\mathrm{mg})$. The ee was determined by modified Mosher ester analysis ${ }^{5}$ to be $96 \%$ ee; $[\alpha]_{\mathrm{D}}{ }^{25}=$ $40.9^{\circ}\left(c 0.64, \mathrm{CHCl}_{3}\right) ;{ }^{1} \mathrm{H} \mathrm{NMR}\left(600 \mathrm{MHz}, \mathrm{CDCl}_{3}\right) \delta 7.62(\mathrm{~d}, J=8.2 \mathrm{~Hz}, 2 \mathrm{H}), 7.41(\mathrm{~d}, J$ $=8.1 \mathrm{~Hz}, 2 \mathrm{H}), 5.77(\mathrm{ddd}, J=17.6,10.0,8.7 \mathrm{~Hz}, 1 \mathrm{H}), 5.39(\mathrm{dq}, J=15.3,6.3 \mathrm{~Hz}, 1 \mathrm{H})$, $5.27(\mathrm{dd}, J=15.4,7.5 \mathrm{~Hz}, 1 \mathrm{H}), 5.21$ (d, $J=10.2 \mathrm{~Hz}, 1 \mathrm{H}), 5.13(\mathrm{~d}, J=17.2 \mathrm{~Hz}, 1 \mathrm{H}), 4.61$ (dd, $J=6.8,2.9 \mathrm{~Hz}, 1 \mathrm{H}), 2.97$ (app. q, $J=7.5 \mathrm{~Hz}, 1 \mathrm{H}), 2.32$ (d, $J=3.1 \mathrm{~Hz}, 1 \mathrm{H}), 1.62$ (d, $J=6.3 \mathrm{~Hz}, 3 \mathrm{H}) .{ }^{13} \mathrm{C} \mathrm{NMR}\left(151 \mathrm{MHz}, \mathrm{CDCl}_{3}\right) \delta 147.6,136.7,132.1,129.4,128.6,127.9$, 
119.3, 119.0, 111.4, 75.9, 55.9, 18.5. $\mathrm{HRMS}\left(\mathrm{API}^{+}\right): m / z$ for $\mathrm{C}_{14} \mathrm{H}_{15} \mathrm{NONa}[\mathrm{M}+\mathrm{Na}]^{+}$calcd. 236.1051 , found 236.1047 .

Methyl 4-((1R,2S,E)-1-hydroxy-2-vinylpent-3-en-1-yl)benzoate (4e)
Prepared according to the general procedure. The crude mixture was hexane and ethyl acetate, 20:1 to 5:1) to give compound $4 \mathbf{e}$ as colorless oil in $97 \%$ yield (23.9 mg). The enantiomeric excess was determined by HPLC analysis to be $98 \%$ ee (254 $\left.\mathrm{nm}, 25{ }^{\circ} \mathrm{C}\right) ; \mathrm{t}_{1}=15.7 \mathrm{~min}, \mathrm{t}_{2}=18.1 \mathrm{~min}[($ Chiralpak IB) hexane $/ i-\mathrm{PrOH}, 95: 5,1.0$ $\mathrm{mL} / \mathrm{min}] ;[\alpha]_{\mathrm{D}}{ }^{25}=49.8^{\circ}\left(c 0.90, \mathrm{CHCl}_{3}\right) ;{ }^{1} \mathrm{H} \mathrm{NMR}\left(600 \mathrm{MHz}, \mathrm{CDCl}_{3}\right) \delta 8.00(\mathrm{~d}, J=8.1$ $\mathrm{Hz}, 2 \mathrm{H}), 7.37$ (d, $J=8.1 \mathrm{~Hz}, 2 \mathrm{H}), 5.78$ (ddd, $J=17.6,10.0,8.5 \mathrm{~Hz}, 1 \mathrm{H}), 5.39$ (dq, $J=$ 15.3, $6.3 \mathrm{~Hz}, 1 \mathrm{H}), 5.28$ (dd, $J=15.4,7.4 \mathrm{~Hz}, 1 \mathrm{H}), 5.20$ (d, $J=10.2 \mathrm{~Hz}, 1 \mathrm{H}), 5.13$ (d, $J=$ $17.2 \mathrm{~Hz}, 1 \mathrm{H}), 4.61(\mathrm{dd}, J=6.8,3.0 \mathrm{~Hz}, 1 \mathrm{H}), 3.91$ (s, 3H), 3.01 (app. q, $J=7.5 \mathrm{~Hz}, 1 \mathrm{H})$, $2.30(\mathrm{~d}, J=3.0 \mathrm{~Hz}, 1 \mathrm{H}), 1.60(\mathrm{~d}, J=6.3 \mathrm{~Hz}, 3 \mathrm{H}) .{ }^{13} \mathrm{C} \mathrm{NMR}\left(151 \mathrm{MHz}, \mathrm{CDCl}_{3}\right) \delta 167.4$, $147.4,137.2,129.6,129.5,129.0,128.9,127.1,118.6,76.2,55.8,52.5,18.5$. HRMS $\left(\mathrm{ESI}^{+}\right): \mathrm{m} / z$ for $\mathrm{C}_{15} \mathrm{H}_{19} \mathrm{O}_{3}[\mathrm{M}+\mathrm{H}]^{+}$calcd. 247.1334, found 247.1325.

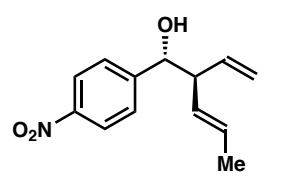

(1R,2S,E)-1-(4-Nitrophenyl)-2-vinylpent-3-en-1-ol

(4f) Prepared according to the general procedure. The crude mixture was purified by flash column chromatography (gradient elution with hexane and ethyl acetate, $20: 1$ to $5: 1)$ to give compound $\mathbf{4 f}$ as colorless oil in $97 \%$ yield (22.6 $\mathrm{mg}$ ). The enantiomeric excess was determined by HPLC analysis to be $99 \%$ ee $\left(254 \mathrm{~nm}, 25{ }^{\circ} \mathrm{C}\right) ; \mathrm{t}_{1}$ $=8.21 \mathrm{~min}, \mathrm{t}_{2}=8.94 \mathrm{~min}\left[(\right.$ Chiralpak ID) hexane $/ i-\mathrm{PrOH}, 95: 5,1.0 \mathrm{~mL} / \mathrm{min}] ;[\alpha]_{\mathrm{D}}{ }^{25}=$ $53.7^{\circ}$ (c 0.83, $\mathrm{CHCl}_{3}$ ); ${ }^{1} \mathrm{H}$ NMR (600 MHz, Chloroform- $d$ ) $\delta 8.19$ (d, $\left.J=8.4 \mathrm{~Hz}, 2 \mathrm{H}\right)$, $7.47(\mathrm{~d}, J=8.5 \mathrm{~Hz}, 2 \mathrm{H}), 5.78$ (ddd, $J=17.6,9.8,8.9 \mathrm{~Hz}, 1 \mathrm{H}), 5.41$ (dq, $J=15.3,6.4 \mathrm{~Hz}$, $1 \mathrm{H}), 5.29(\mathrm{dd}, J=15.4,7.5 \mathrm{~Hz}, 1 \mathrm{H}), 5.22(\mathrm{~d}, J=10.2 \mathrm{~Hz}, 1 \mathrm{H}), 5.14(\mathrm{~d}, J=17.2 \mathrm{~Hz}, 1 \mathrm{H})$, 4.67 (dd, $J=6.7,2.6 \mathrm{~Hz}, 1 \mathrm{H}), 2.99$ (app. q, $J=7.5 \mathrm{~Hz}, 1 \mathrm{H}), 2.36$ (d, $J=2.5 \mathrm{~Hz}, 1 \mathrm{H}$ ), $1.62(\mathrm{~d}, J=6.3 \mathrm{~Hz}, 3 \mathrm{H}) .{ }^{13} \mathrm{C} \mathrm{NMR}\left(151 \mathrm{MHz}, \mathrm{CDCl}_{3}\right) \delta 149.6,147.5,136.6,129.6$, $128.5,128.0,123.5,119.2,75.7,56.0,18.5$. HRMS $\left(\mathrm{ESI}^{+}\right): \mathrm{m} / z$ for $\mathrm{C}_{13} \mathrm{H}_{15} \mathrm{NO}_{3} \mathrm{Na}$ $[\mathrm{M}+\mathrm{Na}]^{+}$calcd. 256.0950, found 256.0943.

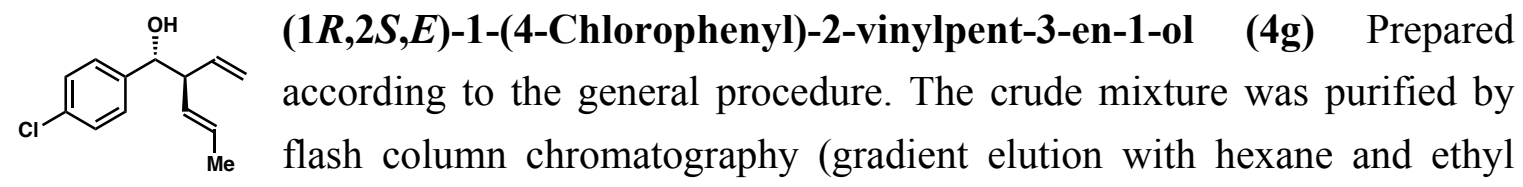
acetate, $20: 1$ to $10: 1)$ to give compound $\mathbf{4 g}$ as colorless oil in $92 \%$ yield $(20.4 \mathrm{mg})$. The enantiomeric excess was determined by HPLC analysis to be $99 \%$ ee $\left(254 \mathrm{~nm}, 25{ }^{\circ} \mathrm{C}\right) ; \mathrm{t}_{1}$ 
$=4.61 \mathrm{~min}, \mathrm{t}_{2}=5.11 \mathrm{~min}\left[(\right.$ Chiralpak IB) hexane $/ i-\operatorname{PrOH}, 95: 5,1.0 \mathrm{~mL} / \mathrm{min}] ;[\alpha]_{\mathrm{D}}{ }^{25}=$ $75.8^{\circ}$ (c 0.72, $\left.\mathrm{CHCl}_{3}\right) ;{ }^{1} \mathrm{H}$ NMR $\left(600 \mathrm{MHz}, \mathrm{CDCl}_{3}\right) \delta 7.30(\mathrm{~d}, J=8.3 \mathrm{~Hz}, 2 \mathrm{H}), 7.23(\mathrm{~d}, J$ $=8.3 \mathrm{~Hz}, 2 \mathrm{H}), 5.78(\mathrm{ddd}, J=17.5,10.0,9.2 \mathrm{~Hz}, 1 \mathrm{H}), 5.39(\mathrm{dq}, \mathrm{d}, J=15.4,6.4 \mathrm{~Hz}, 1 \mathrm{H})$, 5.26 (dd, $J=15.4,7.4 \mathrm{~Hz}, 1 \mathrm{H}), 5.21(\mathrm{~d}, J=10.2 \mathrm{~Hz}, 1 \mathrm{H}), 5.15(\mathrm{~d}, J=17.2 \mathrm{~Hz}, 1 \mathrm{H}), 4.52$ (dd, $J=7.0,2.9 \mathrm{~Hz}, 1 \mathrm{H}), 2.98$ (app. q, $J=7.6 \mathrm{~Hz}, 1 \mathrm{H}), 2.23$ (d, $J=3.0 \mathrm{~Hz}, 1 \mathrm{H}), 1.61$ (d, $J=6.4 \mathrm{~Hz}, 3 \mathrm{H}) .{ }^{13} \mathrm{C} \mathrm{NMR}\left(151 \mathrm{MHz}, \mathrm{CDCl}_{3}\right) \delta 140.6,137.5,133.4,129.0,128.8,128.54$, 128.46, 118.6, 75.9, 55.8, 18.5. HRMS $\left(\mathrm{ESI}^{+}\right): \mathrm{m} / z$ for $\mathrm{C}_{13} \mathrm{H}_{14} \mathrm{Cl}[\mathrm{M}-\mathrm{OH}]^{+}$calcd. 205.0784, found 205.0785.

$(1 R, 2 S, E)-1-(4-B r o m o p h e n y l)-2-v i n y l p e n t-3-e n-1-o l ~(4 h)$ Prepared
according to the general procedure. The crude mixture was purified by
flash column chromatography (gradient elution with hexane and ethyl acetate, $20: 1$ to $10: 1)$ to give compound $\mathbf{4 h}$ as colorless oil in $95 \%$ yield $(25.3 \mathrm{mg})$. The enantiomeric excess was determined by HPLC analysis to be $98 \%$ ee $\left(254 \mathrm{~nm}, 25^{\circ} \mathrm{C}\right) ; \mathrm{t}_{1}$ $=4.83 \mathrm{~min}, \mathrm{t}_{2}=5.53 \mathrm{~min}\left[(\right.$ Chiralpak IB) hexane $/ i-\mathrm{PrOH}, 95: 5,1.0 \mathrm{~mL} / \mathrm{min}] ;[\alpha]_{\mathrm{D}}{ }^{25}=$ $58.8^{\circ}\left(c 0.97, \mathrm{CHCl}_{3}\right) ;{ }^{1} \mathrm{H}$ NMR $\left(600 \mathrm{MHz}, \mathrm{CDCl}_{3}\right) \delta 7.45(\mathrm{~d}, J=8.3 \mathrm{~Hz}, 2 \mathrm{H}), 7.17(\mathrm{~d}, J$ $=8.3 \mathrm{~Hz}, 2 \mathrm{H}), 5.78(\mathrm{ddd}, J=17.4,10.1,8.6 \mathrm{~Hz}, 1 \mathrm{H}), 5.40(\mathrm{dq}, J=15.3,6.3 \mathrm{~Hz}, 1 \mathrm{H})$, 5.26 (dd, $J=15.4,7.4 \mathrm{~Hz}, 1 \mathrm{H}), 5.21(\mathrm{~d}, J=10.2 \mathrm{~Hz}, 1 \mathrm{H}), 5.14(\mathrm{~d}, J=17.2 \mathrm{~Hz}, 1 \mathrm{H}), 4.51$ (dd, $J=7.0,2.9 \mathrm{~Hz}, 1 \mathrm{H}), 2.97$ (app. q, $J=7.5 \mathrm{~Hz}, 1 \mathrm{H}), 2.22$ (d, $J=2.6 \mathrm{~Hz}, 1 \mathrm{H}), 1.61$ (d, $J=6.3 \mathrm{~Hz}, 3 \mathrm{H}) .{ }^{13} \mathrm{C}$ NMR $\left(151 \mathrm{MHz}, \mathrm{CDCl}_{3}\right) \delta 141.2,137.4,131.4,129.0,128.91$, 128.88, 121.6, 118.6, 76.0, 55.8, 18.5. HRMS (ESI $\left.{ }^{+}\right): m / z$ for $\mathrm{C}_{13} \mathrm{H}_{14} \mathrm{Br}[\mathrm{M}-\mathrm{OH}]^{+}$calcd. 249.0279 , found 249.0278

according to the general procedure. The crude mixture was purified by
flash column chromatography (gradient elution with hexane and ethyl acetate, $20: 1$ to 10:1) to give compound $\mathbf{4 i}$ as colorless oil in $95 \%$ yield $(24.4 \mathrm{mg})$. The enantiomeric excess was determined by modified Mosher ester analysis ${ }^{5}$ to be $97 \%$ ee; $[\alpha]_{\mathrm{D}}^{25}=37.0^{\circ}\left(c 0.92, \mathrm{CHCl}_{3}\right) ;{ }^{1} \mathrm{H} \mathrm{NMR}\left(600 \mathrm{MHz}, \mathrm{CDCl}_{3}\right) \delta 7.41(\mathrm{~d}, J=1.5 \mathrm{~Hz}, 1 \mathrm{H})$, 7.39 (d, $J=8.2 \mathrm{~Hz}, 1 \mathrm{H}), 7.12(\mathrm{dd}, J=8.2,1.5 \mathrm{~Hz}, 1 \mathrm{H}), 5.77$ (ddd, $J=17.5,9.4,8.6 \mathrm{~Hz}$, $1 \mathrm{H}), 5.41(\mathrm{dq}, J=15.4,6.3 \mathrm{~Hz}, 1 \mathrm{H}), 5.27$ (dd, $J=15.4,7.4 \mathrm{~Hz}, 1 \mathrm{H}), 5.22(\mathrm{~d}, J=10.2 \mathrm{~Hz}$, $1 \mathrm{H}), 5.15$ (d, $J=17.2 \mathrm{~Hz}, 1 \mathrm{H}), 4.51$ (dd, $J=6.9,2.8 \mathrm{~Hz}, 1 \mathrm{H}$ ), 2.95 (app. q, $J=7.5 \mathrm{~Hz}$, $1 \mathrm{H}), 2.24(\mathrm{~d}, J=2.6 \mathrm{~Hz}, 1 \mathrm{H}), 1.63(\mathrm{~d}, J=6.3 \mathrm{~Hz}, 3 \mathrm{H}) .{ }^{13} \mathrm{C} \mathrm{NMR}\left(151 \mathrm{MHz}, \mathrm{CDCl}_{3}\right) \delta$ $142.5,137.0,132.4,131.5,130.2,129.3,129.1,128.7,126.6,119.0,75.4,55.8,18.5$. HRMS $\left(\mathrm{ESI}^{+}\right): \mathrm{m} / z$ for $\mathrm{C}_{13} \mathrm{H}_{13} \mathrm{Cl}_{2}[\mathrm{M}-\mathrm{OH}]^{+}$calcd. 239.0394, found 239.0402 . 


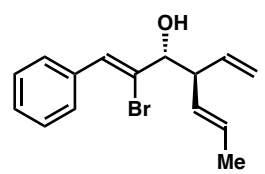

$(1 Z, 3 R, 4 S, 5 E)-2-B r o m o-1-p h e n y l-4-v i n y l h e p t a-1,5-d i e n-3-o l$

Prepared according to the general procedure. The crude mixture was purified by flash column chromatography (gradient elution with hexane and ethyl acetate, $20: 1$ to $10: 1$ ) to give compound $\mathbf{4} \mathbf{j}$ as colorless oil in $93 \%$ yield (27.3 $\mathrm{mg})$. The enantiomeric excess was determined by HPLC analysis to be $99 \%$ ee $(254 \mathrm{~nm}$, $\left.25{ }^{\circ} \mathrm{C}\right) ; \mathrm{t}_{1}=5.35 \mathrm{~min}, \mathrm{t}_{2}=5.68 \mathrm{~min}$ [(Chiralpak IE) hexane $/ i-\mathrm{PrOH}, 95: 5,1.0 \mathrm{~mL} / \mathrm{min}$ ]; $[\alpha]_{\mathrm{D}}^{25}=-18.1^{\circ}\left(c 1.07, \mathrm{CHCl}_{3}\right) ;{ }^{1} \mathrm{H} \mathrm{NMR}\left(600 \mathrm{MHz}, \mathrm{CDCl}_{3}\right) \delta 7.57(\mathrm{~d}, J=7.6 \mathrm{~Hz}, 2 \mathrm{H})$, 7.37 (dd, $J=7.7,7.3 \mathrm{~Hz}, 2 \mathrm{H}), 7.31(\mathrm{dd}, J=7.4,7.3 \mathrm{~Hz}, 1 \mathrm{H}), 7.02(\mathrm{~s}, 1 \mathrm{H}), 5.88$ (ddd, $J=$ $17.4,10.2,8.3 \mathrm{~Hz}, 1 \mathrm{H}), 5.62$ (dq, $J=15.2,6.5 \mathrm{~Hz}, 1 \mathrm{H}), 5.40$ (dd, $J=15.3,7.6 \mathrm{~Hz}, 1 \mathrm{H}$ ), $5.25(\mathrm{~d}, J=10.1 \mathrm{~Hz}, 1 \mathrm{H}), 5.23(\mathrm{~d}, J=17.3 \mathrm{~Hz}, 1 \mathrm{H}), 4.11(\mathrm{dd}, J=7.4,5.1 \mathrm{~Hz}, 1 \mathrm{H}), 3.24$ (app. q, $J=7.8 \mathrm{~Hz}, 1 \mathrm{H}), 2.22(\mathrm{~d}, J=5.0 \mathrm{~Hz}, 1 \mathrm{H}), 1.68(\mathrm{~d}, J=6.5 \mathrm{~Hz}, 3 \mathrm{H}) .{ }^{13} \mathrm{C} \mathrm{NMR}$ $\left(151 \mathrm{MHz}, \mathrm{CDCl}_{3}\right) \delta 136.9,135.5,130.2,129.4,128.9,128.6,128.5,128.4,128.3,118.7$, 79.4, 52.3, 18.6. $\mathrm{HRMS}\left(\mathrm{ESI}^{+}\right): \mathrm{m} / z$ for $\mathrm{C}_{15} \mathrm{H}_{17} \mathrm{OBrNa}[\mathrm{M}+\mathrm{Na}]^{+}$calcd. 315.0360 , found 315.0359 .

Prepared according to the general procedure. The crude mixture was
purified by flash column chromatography (gradient elution with hexane and ethyl acetate, $20: 1$ to $10: 1)$ to give compound $\mathbf{4 k}$ as colorless oil in $85 \%$ yield (17.4 $\mathrm{mg})$. The enantiomeric excess was determined by HPLC analysis to be $98 \%$ ee $(254 \mathrm{~nm}$, $\left.25^{\circ} \mathrm{C}\right) ; \mathrm{t}_{1}=6.30 \mathrm{~min}, \mathrm{t}_{2}=7.05 \mathrm{~min}$ [(Chiralpak IB) hexane $/ i-\mathrm{PrOH}, 95: 5,1.0 \mathrm{~mL} / \mathrm{min}$ ]; $[\alpha]_{\mathrm{D}}^{25}=47.4^{\circ}\left(c 0.57, \mathrm{CHCl}_{3}\right) ;{ }^{1} \mathrm{H} \mathrm{NMR}\left(600 \mathrm{MHz}, \mathrm{CDCl}_{3}\right) \delta 7.34(\mathrm{~s}, 1 \mathrm{H}), 6.43(\mathrm{~d}, J=$ $15.8 \mathrm{~Hz}, 1 \mathrm{H}), 6.37$ (dd, $J=3.1,1.7 \mathrm{~Hz}, 1 \mathrm{H}), 6.23(\mathrm{~d}, J=3.2 \mathrm{~Hz}, 1 \mathrm{H}), 6.17$ (dd, $J=15.8$, $5.9 \mathrm{~Hz}, 1 \mathrm{H}), 5.83$ (ddd, $J=17.3,10.3,7.7 \mathrm{~Hz}, 1 \mathrm{H}), 5.59$ (dq, $J=15.3,6.4 \mathrm{~Hz}, 1 \mathrm{H}), 5.44$ (dd, $J=15.3,8.0 \mathrm{~Hz}, 1 \mathrm{H}), 5.19$ (d, $J=10.3 \mathrm{~Hz}, 1 \mathrm{H}), 5.16$ (d, $J=17.2 \mathrm{~Hz}, 1 \mathrm{H}), 4.19$ (app. q, $J=5.7 \mathrm{~Hz}, 1 \mathrm{H}), 2.94$ (app. q, $J=7.3 \mathrm{~Hz}, 1 \mathrm{H}), 1.86(\mathrm{~d}, J=5.3 \mathrm{~Hz}, 1 \mathrm{H}), 1.71$ (d, $J=6.4$ $\mathrm{Hz}, 3 \mathrm{H}) .{ }^{13} \mathrm{C} \mathrm{NMR}\left(151 \mathrm{MHz}, \mathrm{CDCl}_{3}\right) \delta 152.8,142.2,137.6,129.4,128.9,128.5,119.7$, 117.8, 111.6, 108.4, 74.3, 54.4, 18.6. HRMS $\left(\mathrm{ESI}^{+}\right): m / z$ for $\mathrm{C}_{13} \mathrm{H}_{15} \mathrm{O}[\mathrm{M}-\mathrm{OH}]^{+}$calcd. 187.1123, found 187.1117.

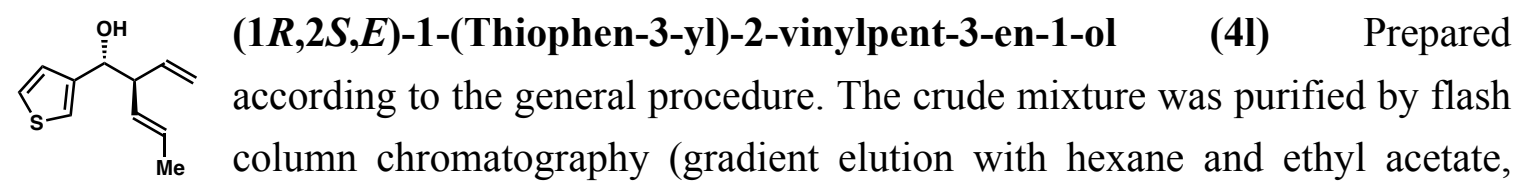

$20: 1$ to $10: 1)$ to give compound $\mathbf{4 l}$ as colorless oil in $84 \%$ yield $(16.3 \mathrm{mg})$. The enantiomeric excess was determined by HPLC analysis to be $96 \%$ ee $\left(254 \mathrm{~nm}, 25^{\circ} \mathrm{C}\right)$; $\mathrm{t}_{1}$ $=5.66 \mathrm{~min}, \mathrm{t}_{2}=6.13 \mathrm{~min}\left[(\right.$ Chiralpak IC) hexane $/ i-\mathrm{PrOH}, 95: 5,1.0 \mathrm{~mL} / \mathrm{min}] ;[\alpha]_{\mathrm{D}}{ }^{25}=$ 
$73.3^{\circ}\left(c 0.57, \mathrm{CHCl}_{3}\right) ;{ }^{1} \mathrm{H} \mathrm{NMR}\left(600 \mathrm{MHz}, \mathrm{CDCl}_{3}\right) \delta 7.28(\mathrm{dd}, J=4.8,3.1 \mathrm{~Hz}, 1 \mathrm{H}), 7.17$ $(\mathrm{d}, J=2.2 \mathrm{~Hz}, 1 \mathrm{H}), 7.04(\mathrm{~d}, J=5.0 \mathrm{~Hz}, 1 \mathrm{H}), 5.81(\mathrm{ddd}, J=17.5,10.1,8.3 \mathrm{~Hz}, 1 \mathrm{H}), 5.47$ $(\mathrm{dq}, J=15.4,6.4 \mathrm{~Hz}, 1 \mathrm{H}), 5.33(\mathrm{dd}, J=15.4,7.4 \mathrm{~Hz}, 1 \mathrm{H}), 5.20(\mathrm{~d}, J=10.2 \mathrm{~Hz}, 1 \mathrm{H})$, $5.16(\mathrm{~d}, J=17.2 \mathrm{~Hz}, 1 \mathrm{H}), 4.68(\mathrm{dd}, J=6.5,4.0 \mathrm{~Hz}, 1 \mathrm{H}), 3.07$ (app. q, $J=7.3 \mathrm{~Hz}, 1 \mathrm{H})$, $2.16(\mathrm{~d}, J=3.9 \mathrm{~Hz}, 1 \mathrm{H}), 1.64(\mathrm{~d}, J=6.4 \mathrm{~Hz}, 3 \mathrm{H}) .{ }^{13} \mathrm{C} \mathrm{NMR}\left(151 \mathrm{MHz}, \mathrm{CDCl}_{3}\right) \delta 143.8$, $137.8,129.3,128.7,126.5,125.8,122.0,118.2,73.0,55.0,18.6$. HRMS $\left(\mathrm{ESI}^{+}\right): \mathrm{m} / z$ for $\mathrm{C}_{11} \mathrm{H}_{13} \mathrm{~S}[\mathrm{M}-\mathrm{OH}]^{+}$calcd. 177.0738, found 177.0725.

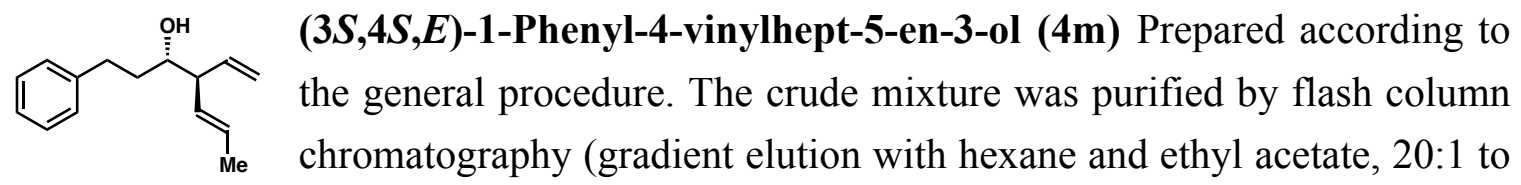
$10: 1)$ to give compound $\mathbf{4 m}$ as colorless oil in $87 \%$ yield $(18.8 \mathrm{mg})$. The enantiomeric excess was determined by HPLC analysis to be $92 \%$ ee $\left(254 \mathrm{~nm}, 25^{\circ} \mathrm{C}\right) ; \mathrm{t}_{1}=5.87 \mathrm{~min}, \mathrm{t}_{2}$ $=8.02 \mathrm{~min}\left[(\right.$ Chiralpak IC) hexane $/ i-\mathrm{PrOH}, 97: 3,1.0 \mathrm{~mL} / \mathrm{min}] ;[\alpha]_{\mathrm{D}}{ }^{25}=0.94$ (c 0.64 , $\left.\mathrm{CHCl}_{3}\right) ;{ }^{1} \mathrm{H} \mathrm{NMR}\left(600 \mathrm{MHz}, \mathrm{CDCl}_{3}\right) \delta 7.29(\mathrm{dd}, J=7.6,7.4 \mathrm{~Hz}, 2 \mathrm{H}), 7.21(\mathrm{~d}, J=7.6 \mathrm{~Hz}$, 2H), 7.18 (dd, $J=7.4,7.3 \mathrm{~Hz}, 1 \mathrm{H}), 5.78$ (ddd, $J=17.7,9.8,8.9 \mathrm{~Hz}, 1 \mathrm{H}), 5.54$ (dq, $J=$ $15.2,6.4 \mathrm{~Hz}, 1 \mathrm{H}), 5.38(\mathrm{dd}, J=15.3,8.0 \mathrm{~Hz}, 1 \mathrm{H}), 5.16(\mathrm{~d}, J=10.3 \mathrm{~Hz}, 1 \mathrm{H}), 5.13(\mathrm{~d}, J=$ $17.6 \mathrm{~Hz}, 1 \mathrm{H}), 3.49-3.53(\mathrm{~m}, 1 \mathrm{H}), 2.86(\mathrm{ddd}, J=14.5,10.6,5.0 \mathrm{~Hz}, 1 \mathrm{H}), 2.77$ (app. q, $J$ $=7.7 \mathrm{~Hz}, 1 \mathrm{H}), 2.65(\mathrm{ddd}, J=13.8,10.2,6.6 \mathrm{~Hz}, 1 \mathrm{H}), 1.85(\mathrm{dddd}, J=13.5,9.7,6.5,2.7$ $\mathrm{Hz}, 1 \mathrm{H}), 1.72(\mathrm{~d}, J=4.3 \mathrm{~Hz}, 1 \mathrm{H}), 1.69(\mathrm{~d}, J=6.4 \mathrm{~Hz}, 3 \mathrm{H}), 1.62-1.69(\mathrm{~m}, 1 \mathrm{H}) .{ }^{13} \mathrm{C}$ NMR $\left(151 \mathrm{MHz}, \mathrm{CDCl}_{3}\right) \delta 142.6,138.0,129.9,128.8,128.7,128.4,126.1,117.5,72.9$, 54.6, 36.3, 32.5, 18.6. HRMS $\left(\mathrm{ESI}^{+}\right): \mathrm{m} / z$ for $\mathrm{C}_{15} \mathrm{H}_{24} \mathrm{NO}\left[\mathrm{M}+\mathrm{NH}_{4}\right]^{+}$calcd. 234.1858, found 234.1847 .

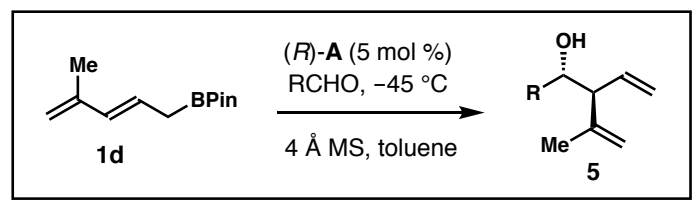

General procedure for the syntheses of homoallylic alcohols 5: To a reaction flask containing a stir bar and freshly activated $4 \AA \mathrm{MS}(50 \mathrm{mg})$ was added phosphoric acid $(R)$-A $(3.8 \mathrm{mg}, 0.005 \mathrm{mmol})$. Toluene $(0.3 \mathrm{~mL})$ was added to the flask followed by drop wise addition of freshly distilled aldehyde $(0.1 \mathrm{mmol}$, if it is a liquid $)$. The mixture was placed in a $-45{ }^{\circ} \mathrm{C}$ cold bath and stirred for $5 \mathrm{~min}$. Then allylboronate $1 \mathrm{~d}(0.13 \mathrm{mmol})$ was added slowly to the reaction mixture via a microliter syringe. The mixture was kept at $-45{ }^{\circ} \mathrm{C}$ and stirred for $48 \mathrm{~h}$. After complete consumption of the aldehyde, sat. $\mathrm{NaHCO}_{3}$ $(1.0 \mathrm{~mL})$ was added to the reaction mixture followed by slow addition of $30 \% \mathrm{H}_{2} \mathrm{O}_{2}(0.5$ 
$\mathrm{mL})$ at $0{ }^{\circ} \mathrm{C}$. The reaction was stirred vigorously for $3 \mathrm{~h}$; then diethyl ether $(1 \mathrm{~mL})$ and brine $(1 \mathrm{~mL})$ was added. The organic layer was separated and the aqueous layer was extracted with $\mathrm{Et}_{2} \mathrm{O}(3 \times 2 \mathrm{~mL})$. The combined organic extracts were dried over anhydrous sodium sulfate, filtered, and concentrated under reduced pressure. Purification of the crude product was performed by flash chromatography (gradient elution with hexane and ethyl acetate) to give product 5.

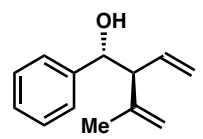

$(1 R, 2 S)-3-M e t h y l-1-p h e n y l-2-v i n y l b u t-3-e n-1-o l ~(5 a)$ Prepared according to the general procedure. The crude mixture was purified by flash column chromatography (gradient elution with hexane and ethyl acetate, 20:1 to $10: 1)$ to give compound $\mathbf{5 a}$ as colorless oil in $89 \%$ yield $(16.8 \mathrm{mg})$. The enantiomeric excess was determined by HPLC analysis to be $94 \%$ ee $\left(254 \mathrm{~nm}, 25{ }^{\circ} \mathrm{C}\right) ; \mathrm{t}_{1}=5.98 \mathrm{~min}, \mathrm{t}_{2}$ $=6.64 \mathrm{~min}\left[(\right.$ Chiralpak IE) hexane $/ i-\mathrm{PrOH}, 95: 5,1.0 \mathrm{~mL} / \mathrm{min}] ;[\alpha]_{\mathrm{D}}{ }^{25}=83.8^{\circ}(c 0.74$, $\left.\mathrm{CHCl}_{3}\right) ;{ }^{1} \mathrm{H}$ NMR $\left(600 \mathrm{MHz}, \mathrm{CDCl}_{3}\right) \delta 7.26-7.28(\mathrm{~m}, 4 \mathrm{H}), 7.21-7.23(\mathrm{~m}, 1 \mathrm{H}), 5.95$ (ddd, $J=17.6,9.7,9.5 \mathrm{~Hz}, 1 \mathrm{H}), 5.16(\mathrm{~d}, J=10.1 \mathrm{~Hz}, 1 \mathrm{H}), 5.13(\mathrm{~d}, J=17.2 \mathrm{~Hz}, 1 \mathrm{H}), 4.72$ (s, 1H), 4.67 (s, 1H), 4.64 (d, $J=7.6 \mathrm{~Hz}, 1 \mathrm{H}), 2.93$ (dd, $J=8.5,8.5 \mathrm{~Hz}, 1 \mathrm{H}), 2.19(\mathrm{~s}, 1 \mathrm{H})$, 1.54 (s, 3H). ${ }^{13} \mathrm{C}$ NMR (151 MHz, $\left.\mathrm{CDCl}_{3}\right) \delta 144.8,142.2,137.4,128.4,127.9,127.1$, 118.6, 113.5, 75.2, 60.4, 22.2. HRMS $\left(\mathrm{ESI}^{+}\right): \mathrm{m} / \mathrm{z}$ for $\mathrm{C}_{13} \mathrm{H}_{17} \mathrm{O}[\mathrm{M}+\mathrm{H}]^{+}$calcd. 189.1279, found 189.1256 .

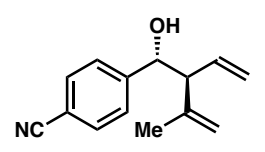

4-((1R,2S)-1-Hydroxy-3-methyl-2-vinylbut-3-en-1-yl)benzonitrile (5b) Prepared according to the general procedure. The crude mixture was purified by flash column chromatography (gradient elution with hexane and ethyl acetate, $20: 1$ to $5: 1)$ to give compound $\mathbf{5 b}$ as colorless oil in $95 \%$ yield $(20.3$ $\mathrm{mg})$. The enantiomeric excess was determined by HPLC analysis to be $98 \%$ ee $(254 \mathrm{~nm}$, $\left.25{ }^{\circ} \mathrm{C}\right) ; \mathrm{t}_{1}=12.5 \mathrm{~min}, \mathrm{t}_{2}=13.2 \mathrm{~min}$ [(Chiralpak IE) hexane $/ i-\mathrm{PrOH}, 95: 5,1.0 \mathrm{~mL} / \mathrm{min}$ ]; $[\alpha]_{\mathrm{D}}{ }^{25}=50.5^{\circ}\left(c 0.92, \mathrm{CHCl}_{3}\right) ;{ }^{1} \mathrm{H}$ NMR $\left(600 \mathrm{MHz}, \mathrm{CDCl}_{3}\right) \delta 7.62(\mathrm{~d}, J=8.1 \mathrm{~Hz}, 2 \mathrm{H})$, $7.44(\mathrm{~d}, J=8.0 \mathrm{~Hz}, 2 \mathrm{H}), 5.94(\mathrm{ddd}, J=17.0,9.8,9.6 \mathrm{~Hz}, 1 \mathrm{H}), 5.22(\mathrm{~d}, J=10.1 \mathrm{~Hz}, 1 \mathrm{H})$, $5.13(\mathrm{~d}, J=17.1 \mathrm{~Hz}, 1 \mathrm{H}), 4.84(\mathrm{~s}, 1 \mathrm{H}), 4.76$ (d, $J=7.0 \mathrm{~Hz}, 1 \mathrm{H}), 4.73$ (s, 1H), 2.89 (dd, $J$ $=8.2,8.2 \mathrm{~Hz}, 1 \mathrm{H}), 2.32(\mathrm{~s}, 1 \mathrm{H}), 1.64(\mathrm{~s}, 3 \mathrm{H}) .{ }^{13} \mathrm{C} \mathrm{NMR}\left(151 \mathrm{MHz}, \mathrm{CDCl}_{3}\right) \delta 147.6,144.1$, 136.1, 132.3, 127.7, 119.6, 119.3, 114.3, 111.5, 74.2, 60.4, 22.3. HRMS (ESI $\left.{ }^{+}\right): m / z$ for $\mathrm{C}_{14} \mathrm{H}_{16} \mathrm{NO}[\mathrm{M}+\mathrm{H}]^{+}$calcd. 214.1232, found 214.1240.

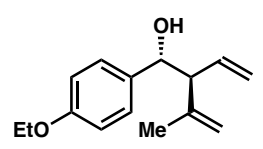

(1R,2S)-1-(4-Ethoxyphenyl)-3-methyl-2-vinylbut-3-en-1-ol

Prepared according to the general procedure. The crude mixture was purified by flash column chromatography (gradient elution with hexane 
and ethyl acetate, $20: 1$ to $10: 1)$ to give compound $\mathbf{5 c}$ as colorless oil in $77 \%$ yield (17.9 $\mathrm{mg})$. The enantiomeric excess was determined by HPLC analysis to be $94 \%$ ee $(254 \mathrm{~nm}$, $\left.25^{\circ} \mathrm{C}\right) ; \mathrm{t}_{1}=9.86 \mathrm{~min}, \mathrm{t}_{2}=10.9 \min$ [(Chiralpak IA) hexane $/ i-\mathrm{PrOH}, 95: 5,1.0 \mathrm{~mL} / \mathrm{min}$ ]; $[\alpha]_{\mathrm{D}}{ }^{25}=59.4^{\circ}\left(c 0.80, \mathrm{CHCl}_{3}\right) ;{ }^{1} \mathrm{H} \mathrm{NMR}\left(600 \mathrm{MHz}, \mathrm{CDCl}_{3}\right) \delta 7.23(\mathrm{~d}, J=8.2 \mathrm{~Hz}, 2 \mathrm{H})$, $6.84(\mathrm{~d}, J=8.3 \mathrm{~Hz}, 2 \mathrm{H}), 6.00$ (ddd, $J=18.1,10.0,9.2 \mathrm{~Hz}, 1 \mathrm{H}), 5.22(\mathrm{~d}, J=9.1 \mathrm{~Hz}, 1 \mathrm{H})$, $5.20(\mathrm{~d}, J=16.3 \mathrm{~Hz}, 1 \mathrm{H}), 4.74(\mathrm{~s}, 1 \mathrm{H}), 4.70(\mathrm{~s}, 1 \mathrm{H}), 4.62(\mathrm{~d}, J=8.1 \mathrm{~Hz}, 1 \mathrm{H}), 4.01(\mathrm{q}, J=$ $6.9 \mathrm{~Hz}, 2 \mathrm{H}), 2.96$ (dd, $J=8.7,8.6 \mathrm{~Hz}, 1 \mathrm{H}), 2.17$ (s, 1H), 1.57 (s, 3H), 1.40 (t, $J=6.9 \mathrm{~Hz}$, $3 \mathrm{H}) .{ }^{13} \mathrm{C}$ NMR $\left(151 \mathrm{MHz}, \mathrm{CDCl}_{3}\right) \delta 158.6,144.9,137.8,134.2,128.3,118.4,114.3$, 113.4, 74.9, 63.6, 60.4, 22.1, 15.2. HRMS $\left(\mathrm{EI}^{+}\right): m / z$ for $\mathrm{C}_{15} \mathrm{H}_{18} \mathrm{O}\left[\mathrm{M}-\mathrm{H}_{2} \mathrm{O}\right]^{+}$calcd. 214.1358 , found 214.1379 .

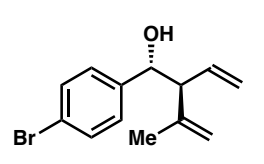

(1R,2S)-1-(4-Bromophenyl)-3-methyl-2-vinylbut-3-en-1-ol

Prepared according to the general procedure. The crude mixture was purified by flash column chromatography (gradient elution with hexane and ethyl acetate, $20: 1$ to $10: 1$ ) to give compound $\mathbf{5 d}$ as colorless oil in $80 \%$ yield (21.4 $\mathrm{mg})$. The enantiomeric excess was determined by HPLC analysis to be $94 \%$ ee $(254 \mathrm{~nm}$, $\left.25{ }^{\circ} \mathrm{C}\right) ; \mathrm{t}_{1}=6.21 \mathrm{~min}, \mathrm{t}_{2}=6.78 \mathrm{~min}$ [(Chiralpak IB) hexane $/ i-\mathrm{PrOH}, 97: 3,1.0 \mathrm{~mL} / \mathrm{min}$ ]; $[\alpha]_{\mathrm{D}}{ }^{25}=29.8^{\circ}\left(c 0.90, \mathrm{CHCl}_{3}\right) ;{ }^{1} \mathrm{H} \mathrm{NMR}\left(600 \mathrm{MHz}, \mathrm{CDCl}_{3}\right) \delta 7.44(\mathrm{~d}, J=8.3 \mathrm{~Hz}, 2 \mathrm{H})$, $7.20(\mathrm{~d}, J=8.2 \mathrm{~Hz}, 2 \mathrm{H}), 5.96(\mathrm{ddd}, J=17.0,9.8,9.6 \mathrm{~Hz}, 1 \mathrm{H}), 5.22(\mathrm{dd}, J=10.1,1.0 \mathrm{~Hz}$, $1 \mathrm{H}), 5.16(\mathrm{~d}, J=17.1 \mathrm{~Hz}, 1 \mathrm{H}), 4.80(\mathrm{~s}, 1 \mathrm{H}), 4.72(\mathrm{~s}, 1 \mathrm{H}), 4.66(\mathrm{~d}, J=7.6 \mathrm{~Hz}, 1 \mathrm{H}), 2.90$ (dd, $J=8.5,8.4 \mathrm{~Hz}, 1 \mathrm{H}), 2.22(\mathrm{~s}, 1 \mathrm{H}), 1.61(\mathrm{~s}, 3 \mathrm{H}) .{ }^{13} \mathrm{C} \mathrm{NMR}\left(151 \mathrm{MHz}, \mathrm{CDCl}_{3}\right) \delta 144.4$, $141.2,136.9,131.5,128.8,121.7,119.1,113.8,74.5,60.4,22.3$. HRMS $\left(\mathrm{CI}^{+}\right): \mathrm{m} / \mathrm{z}$ for $\mathrm{C}_{13} \mathrm{H}_{16} \mathrm{BrO}[\mathrm{M}+\mathrm{H}]^{+}$calcd. 267.0385, found 267.0387.

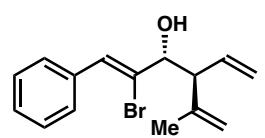

(3R,4S,Z)-2-Bromo-5-methyl-1-phenyl-4-vinylhexa-1,5-dien-3-ol

(5e) Prepared according to the general procedure. The crude mixture was purified by flash column chromatography (gradient elution with hexane and ethyl acetate, 20:1 to 10:1) to give compound 5e as colorless oil in 93\% yield $(27.2 \mathrm{mg}$ ). The enantiomeric excess was determined by HPLC analysis to be $99 \%$ ee (254 $\left.\mathrm{nm}, 25{ }^{\circ} \mathrm{C}\right) ; \mathrm{t}_{1}=8.71 \mathrm{~min}, \mathrm{t}_{2}=10.4 \mathrm{~min}$ [(Chiralpak IA) hexane/i-PrOH, 95:5, 1.0 $\mathrm{mL} / \mathrm{min}] ;[\alpha]_{\mathrm{D}}{ }^{25}=-5.7^{\circ}\left(c 1.26, \mathrm{CHCl}_{3}\right) ;{ }^{1} \mathrm{H} \mathrm{NMR}\left(600 \mathrm{MHz}, \mathrm{CDCl}_{3}\right) \delta 7.57(\mathrm{~d}, J=7.4$ $\mathrm{Hz}, 2 \mathrm{H}), 7.36$ (dd, $J=7.4,7.3 \mathrm{~Hz}, 2 \mathrm{H}), 7.31$ (dd, $J=7.0,7.0 \mathrm{~Hz}, 1 \mathrm{H}), 7.02(\mathrm{~s}, 1 \mathrm{H}), 5.97$ (ddd, $J=18.0,9.4,9.3 \mathrm{~Hz}, 1 \mathrm{H}), 5.25-5.28(\mathrm{~m}, 2 \mathrm{H}), 4.92(\mathrm{~s}, 1 \mathrm{H}), 4.91(\mathrm{~s}, 1 \mathrm{H}), 4.31(\mathrm{dd}$, $J=7.1,4.6 \mathrm{~Hz}, 1 \mathrm{H}), 3.25(\mathrm{dd}, J=8.3,8.3 \mathrm{~Hz}, 1 \mathrm{H}), 2.21(\mathrm{~d}, J=4.0 \mathrm{~Hz}, 1 \mathrm{H}), 1.78(\mathrm{~s}, 3 \mathrm{H})$. ${ }^{13} \mathrm{C}$ NMR $\left(151 \mathrm{MHz}, \mathrm{CDCl}_{3}\right) \delta 144.0,136.0,135.4,130.1,129.4,128.4$ (2 overlapping 
${ }^{13} \mathrm{C}$ signals), 128.0, 119.2, 114.3, 77.7, 56.7, 21.2. HRMS (ESI $\left.{ }^{+}\right): m / z$ for $\mathrm{C}_{15} \mathrm{H}_{17} \mathrm{ONaBr}$ $[\mathrm{M}+\mathrm{Na}]^{+}$calcd. 315.0360, found 315.0362.

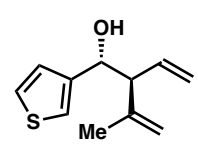

(1R,2S)-3-Methyl-1-(thiophen-3-yl)-2-vinylbut-3-en-1-ol (5f) Prepared according to the general procedure. The crude mixture was purified by flash column chromatography (gradient elution with hexane and ethyl acetate, $20: 1$ to $10: 1)$ to give compound $\mathbf{5 f}$ as colorless oil in $91 \%$ yield (17.7 $\mathrm{mg})$. The enantiomeric excess was determined by HPLC analysis to be $93 \%$ ee $\left(254 \mathrm{~nm}, 25{ }^{\circ} \mathrm{C}\right) ; \mathrm{t}_{1}$ $=6.89 \mathrm{~min}, \mathrm{t}_{2}=7.59 \mathrm{~min}\left[(\right.$ Chiralpak IE) hexane $/ i-\mathrm{PrOH}, 95: 5,1.0 \mathrm{~mL} / \mathrm{min}] ;[\alpha]_{\mathrm{D}}{ }^{25}=$ $68.6^{\circ}$ (c 0.76, $\left.\mathrm{CHCl}_{3}\right) ;{ }^{1} \mathrm{H} \mathrm{NMR}\left(600 \mathrm{MHz}, \mathrm{CDCl}_{3}\right) \delta 7.25-7.26(\mathrm{~m}, 1 \mathrm{H}), 7.18$ (app. s, $1 \mathrm{H}), 7.07$ (d, $J=4.7 \mathrm{~Hz}, 1 \mathrm{H}), 5.98$ (ddd, $J=17.0,9.5,9.5 \mathrm{~Hz}, 1 \mathrm{H}), 5.22(\mathrm{~d}, J=10.4 \mathrm{~Hz}$, 1H), 5.20 (d, J=17.8 Hz, 1H), $4.82-4.84$ (m, 1H), 4.81 (s, 1H), 4.75 (s, 1H), 2.99 (dd, $J$ $=8.5,8.2 \mathrm{~Hz}, 1 \mathrm{H}), 2.21(\mathrm{~d}, J=1.6 \mathrm{~Hz}, 1 \mathrm{H}), 1.63(\mathrm{~s}, 3 \mathrm{H}) .{ }^{13} \mathrm{C} \mathrm{NMR}\left(151 \mathrm{MHz}, \mathrm{CDCl}_{3}\right) \delta$

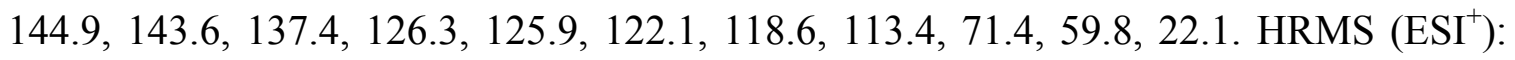
$m / z$ for $\mathrm{C}_{11} \mathrm{H}_{15} \mathrm{OS}[\mathrm{M}+\mathrm{H}]^{+}$calcd. 195.0844, found 195.0838 .

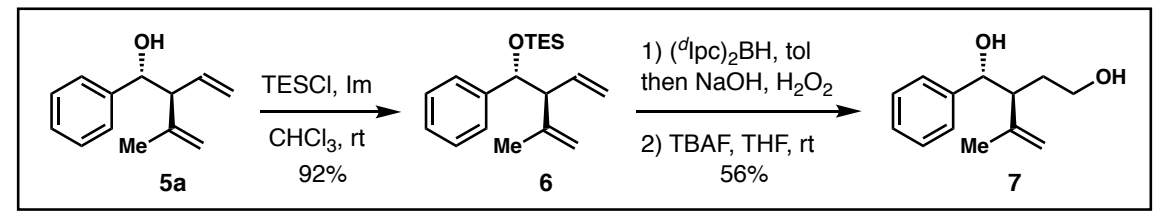

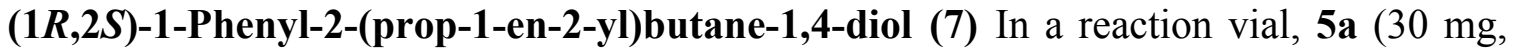
$0.16 \mathrm{mmol}, 1.0$ equiv), imidazole ( $22 \mathrm{mg}, 0.32 \mathrm{mmol}, 2.0$ equiv) and TESCl (48 mg, 0.32 mmol, 2.0 equiv) were dissolved in $\mathrm{CHCl}_{3}(2 \mathrm{~mL})$. The reaction mixture was stirred at ambient temperature for $1 \mathrm{~h}$. After complete consumption of $\mathbf{5 a}$, the reaction was diluted with diethyl ether $(2 \mathrm{~mL})$, and the resulting mixture was filtered through a pad of silica gel. The filtrate was concentrated under reduced pressure to provide the crude product, which was purified by flash column chromatography (hexane : ethyl acetate $=100: 1$ ) to give product 6 (44 mg, 92\% yield) as colorless oil. ${ }^{1} \mathrm{H}$ NMR $\left(600 \mathrm{MHz}, \mathrm{CDCl}_{3}\right) \delta 7.26-$ 7.29 (m, 4H), $7.23-7.26(\mathrm{~m}, 1 \mathrm{H}), 6.09$ (ddd, $J=17.1,9.5,9.2 \mathrm{~Hz}, 1 \mathrm{H}), 5.08(\mathrm{~d}, J=10.3$ $\mathrm{Hz}, 1 \mathrm{H}), 5.00(\mathrm{~d}, J=17.1 \mathrm{~Hz}, 1 \mathrm{H}), 4.73(\mathrm{~s}, 1 \mathrm{H}), 4.70(\mathrm{~d}, J=7.2 \mathrm{~Hz}, 1 \mathrm{H}), 4.65(\mathrm{~s}, 1 \mathrm{H})$, $2.97(\mathrm{dd}, J=7.9,7.8 \mathrm{~Hz}, 1 \mathrm{H}), 1.63(\mathrm{~s}, 3 \mathrm{H}), 0.86(\mathrm{t}, J=8.0 \mathrm{~Hz}, 9 \mathrm{H}), 0.44-0.54(\mathrm{~m}, 6 \mathrm{H})$. ${ }^{13} \mathrm{C}$ NMR $\left(151 \mathrm{MHz}, \mathrm{CDCl}_{3}\right) \delta 145.6,144.2,138.2,127.9,127.4,127.2,116.4,113.2$, 77.2, 60.6, 22.1, 7.1, 5.1.

In a glove box, $\left({ }^{d} \mathrm{Ipc}\right){ }_{2} \mathrm{BH}(34 \mathrm{mg}, 0.12 \mathrm{mmol}, 1.2$ equiv) was added to a solution of compound 6 (30 mg, $0.1 \mathrm{mmol}, 1.0$ equiv) in anhydrous toluene $(0.5 \mathrm{~mL})$. The reaction mixture was stirred for $2 \mathrm{~h}$ at ambient temperature, and was removed from glove box. 
Then $3 \mathrm{~N} \mathrm{NaOH}(0.5 \mathrm{~mL})$ was added to the reaction followed by slow addition of $30 \%$ $\mathrm{H}_{2} \mathrm{O}_{2}(0.5 \mathrm{~mL})$ at $0{ }^{\circ} \mathrm{C}$. The reaction mixture was stirred at ambient temperature for $12 \mathrm{~h}$. Then brine $(1 \mathrm{~mL})$ and $\mathrm{Et}_{2} \mathrm{O}(0.5 \mathrm{~mL})$ were added. The organic layer was separated, and the aqueous layer was extracted with $\mathrm{Et}_{2} \mathrm{O}(3 \mathrm{x} 1 \mathrm{~mL})$. The combined organic extracts were dried over anhydrous magnesium sulfate, filtered, and concentrated under reduced pressure. The resulting crude product was dissolved in THF $(2 \mathrm{~mL})$, and TBAF $(1.0 \mathrm{M}$ solution in THF, $0.2 \mathrm{~mL}$ ) was added. The reaction mixture was stirred at ambient temperature for $4 \mathrm{~h}$. Then brine $(2 \mathrm{~mL})$ and $\mathrm{Et}_{2} \mathrm{O}(2 \mathrm{~mL})$ were added. The organic layer was separated and the aqueous layer was extracted with $\mathrm{Et}_{2} \mathrm{O}(3 \times 2 \mathrm{~mL})$. The combined organic extracts were dried over anhydrous magnesium sulfate, filtered, and concentrated under reduced pressure. The crude product was purified by flash column chromatography (gradient elution with hexane and ethyl acetate, 10:1 to 1:1) to give product 7 (11.5 mg, $56 \%$ yield) as colorless oil. ${ }^{1} \mathrm{H}$ NMR $\left(600 \mathrm{MHz}, \mathrm{CDCl}_{3}\right) \delta 7.32-7.33(\mathrm{~m}, 4 \mathrm{H}), 7.26-$ $7.28(\mathrm{~m}, 1 \mathrm{H}), 4.76(\mathrm{~s}, 1 \mathrm{H}), 4.70(\mathrm{~s}, 1 \mathrm{H}), 4.65(\mathrm{~d}, J=7.4 \mathrm{~Hz}, 1 \mathrm{H}), 3.70$ (ddd, $J=10.9,5.6$, $5.5 \mathrm{~Hz}, 1 \mathrm{H}), 3.56-3.60(\mathrm{~m}, 1 \mathrm{H}), 2.53-2.57(\mathrm{~m}, 1 \mathrm{H}), 1.93-2.01(\mathrm{~m}, 1 \mathrm{H}), 1.96(\mathrm{br}, 2 \mathrm{H})$, $1.78-1.83(\mathrm{~m}, 1 \mathrm{H}), 1.60(\mathrm{~s}, 3 \mathrm{H}) .{ }^{13} \mathrm{C} \mathrm{NMR}\left(151 \mathrm{MHz}, \mathrm{CDCl}_{3}\right) \delta$ 145.8, 143.2, 128.6, 127.9, 126.9, 113.6, 76.4, 62.0, 52.2, 32.4, 21.6. HRMS $\left(\mathrm{ESI}^{+}\right): m / z$ for $\mathrm{C}_{13} \mathrm{H}_{18} \mathrm{O}_{2} \mathrm{Na}$ $[\mathrm{M}+\mathrm{Na}]^{+}$calcd. 229.1204, found 229.1193.

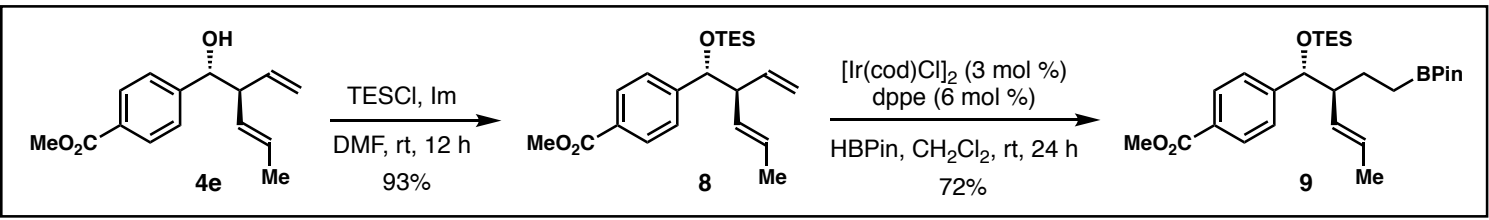

Methyl-4-((1R,2S,E)-2-(2-(4,4,5,5-tetramethyl-1,3,2-dioxaborolan-2-yl)ethyl)-1-((trie thylsilyl)oxy)pent-3-en-1-yl)benzoate (9) In a reaction vial, $4 \mathbf{e}(49 \mathrm{mg}, 0.2 \mathrm{mmol}, 1.0$ equiv), imidazole (27 mg, $0.4 \mathrm{mmol}, 2.0$ equiv) and TESCl (60 mg, $0.4 \mathrm{mmol}, 2.0$ equiv) were dissolved in DMF $(1 \mathrm{~mL})$. The reaction mixture was stirred at ambient temperature for $12 \mathrm{~h}$. After complete consumption of $\mathbf{4 e}$, the reaction was diluted with water $(5 \mathrm{~mL})$ and the resulting solution was extracted with diethyl ether $(3 \times 3 \mathrm{~mL})$. The combined organic layers were dried over anhydrous sodium sulfate, filtered, and concentrated under reduced pressure. Purification of the crude product was performed by flash column chromatography (gradient elution with hexane and ethyl acetate, 100:1 to 20:1) to give product 8 (67 mg, 93\% yield) as colorless oil. ${ }^{1} \mathrm{H}$ NMR (600 MHz, Chloroform- $d$ ) $\delta 7.95$ $(\mathrm{d}, J=8.3 \mathrm{~Hz}, 2 \mathrm{H}), 7.31(\mathrm{~d}, J=8.3 \mathrm{~Hz}, 2 \mathrm{H}), 5.81$ (ddd, $J=17.7,9.8,8.3 \mathrm{~Hz}, 1 \mathrm{H}), 5.39$ $(\mathrm{dd}, J=15.6,7.2 \mathrm{~Hz}, 1 \mathrm{H}), 5.29-5.35(\mathrm{~m}, 1 \mathrm{H}), 5.00(\mathrm{~d}, J=10.4 \mathrm{~Hz}, 1 \mathrm{H}), 4.86(\mathrm{~d}, J=$ 
$17.3 \mathrm{~Hz}, 1 \mathrm{H}), 4.65(\mathrm{~d}, J=5.4 \mathrm{~Hz}, 1 \mathrm{H}), 3.90(\mathrm{~s}, 3 \mathrm{H}), 2.89-2.92(\mathrm{~m}, 1 \mathrm{H}), 1.60(\mathrm{~d}, J=5.8$ $\mathrm{Hz}, 3 \mathrm{H}), 0.85(\mathrm{t}, J=7.9 \mathrm{~Hz}, 9 \mathrm{H}), 0.45-0.54(\mathrm{~m}, 6 \mathrm{H}) .{ }^{13} \mathrm{C} \mathrm{NMR}\left(151 \mathrm{MHz}, \mathrm{CDCl}_{3}\right) \delta$ 167.6, 149.4, 137.6, 130.6, 129.3, 128.9, 127.3, 127.0, 116.6, 78.1, 56.2, 52.4, 18.5, 7.1, 5.0.

In a glove box, to a 2-dram reaction vial containing a stirring bar were added $[\operatorname{Ir}(\operatorname{cod}) \mathrm{Cl}]_{2}(2.0 \mathrm{mg}, 0.003 \mathrm{mmol}, 3 \mathrm{~mol} \%)$ and dppe $(2.4 \mathrm{mg}, 0.006 \mathrm{mmol}, 6 \mathrm{~mol} \%)$. The reaction vial was removed from glove box, and $\mathrm{CH}_{2} \mathrm{Cl}_{2}(0.2 \mathrm{~mL})$, HBPin $(19 \mathrm{mg}, 0.15$ mmol, 1.5 equiv), compound 8 ( $36 \mathrm{mg}, 0.1 \mathrm{mmol}, 1.0$ equiv) in $0.3 \mathrm{~mL} \mathrm{CH}_{2} \mathrm{Cl}_{2}$ were added sequentially to the reaction vial. The resulting mixture was stirred at ambient temperature for $24 \mathrm{~h}$. After complete consumption of compound $\mathbf{8}$, methanol $(1 \mathrm{~mL})$ and water $(3 \mathrm{~mL})$ were added. The resulting mixture was extracted with $\mathrm{Et}_{2} \mathrm{O}(3 \times 3 \mathrm{~mL})$. The combined organic layers were dried over anhydrous sodium sulfate, filtered, and concentrated under reduced pressure. Purification of the crude product was performed by flash column chromatography (gradient elution with hexane and ethyl acetate, 100:1 to 20:1) to give product 9 (35 mg, 72\% yield) as colorless oil. ${ }^{1} \mathrm{H}$ NMR (600 MHz, $\left.\mathrm{CDCl}_{3}\right) \delta 7.93$ (d, $J=$ $8.3 \mathrm{~Hz}, 2 \mathrm{H}), 7.30$ (d, $J=8.3 \mathrm{~Hz}, 2 \mathrm{H}), 5.20$ (dq, $J=13.0,6.4 \mathrm{~Hz}, 1 \mathrm{H}), 5.05$ (dd, $J=15.4$, $9.3 \mathrm{~Hz}, 1 \mathrm{H}), 4.55(\mathrm{~d}, J=5.9 \mathrm{~Hz}, 1 \mathrm{H}), 3.90(\mathrm{~s}, 3 \mathrm{H}), 2.08-2.13(\mathrm{~m}, 1 \mathrm{H}), 1.62-1.69(\mathrm{~m}$, $1 \mathrm{H}), 1.54(\mathrm{~d}, J=6.2 \mathrm{~Hz}, 3 \mathrm{H}), 1.22-1.25(\mathrm{~m}, 1 \mathrm{H}), 1.21(\mathrm{~s}, 12 \mathrm{H}), 0.83-0.86(\mathrm{~m}, 9 \mathrm{H})$, 0.75 (ddd, $J=15.4,9.8,5.4 \mathrm{~Hz}, 1 \mathrm{H}), 0.53-0.60(\mathrm{~m}, 1 \mathrm{H}), 0.43-0.52(\mathrm{~m}, 6 \mathrm{H}) .{ }^{13} \mathrm{C}$ NMR $\left(101 \mathrm{MHz}, \mathrm{CDCl}_{3}\right) \delta 167.6,150.1,131.8,129.2,128.8,127.8,127.2,83.1,78.4,54.2$, 52.3, 25.2, 25.1, 18.3, 7.1, 5.2. HRMS $\left(\mathrm{ESI}^{+}\right): \mathrm{m} / z$ for $\mathrm{C}_{27} \mathrm{H}_{45} \mathrm{BO}_{5} \mathrm{SiNa}[\mathrm{M}+\mathrm{Na}]^{+}$calcd. 511.3027, found 511.3014.

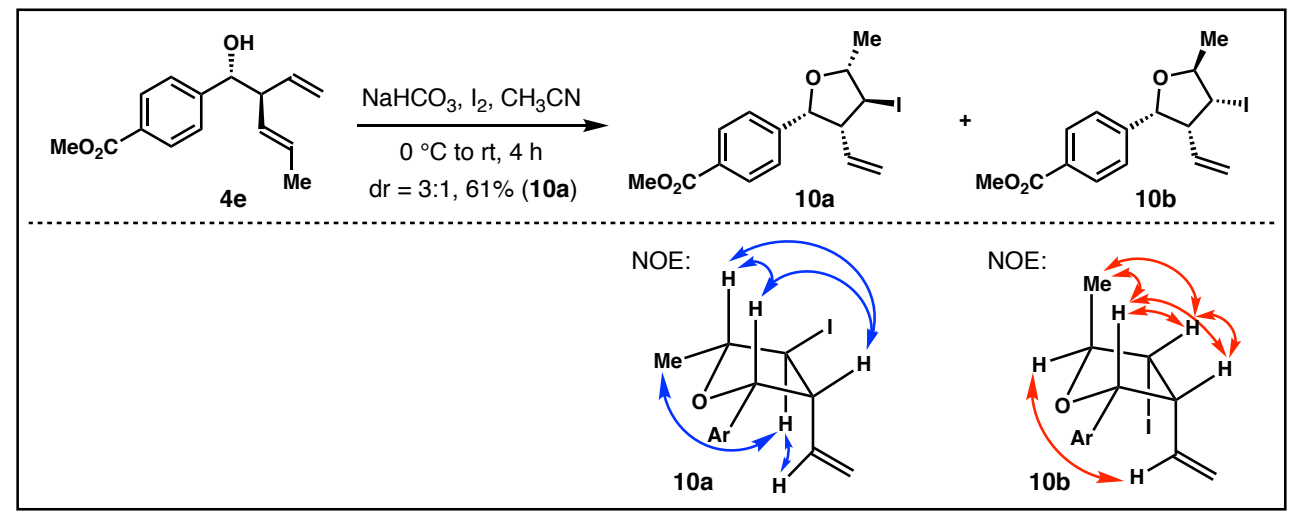

To a solution of alcohol $4 \mathbf{e}(12 \mathrm{mg}, 0.05 \mathrm{mmol})$ in anhydrous $\mathrm{CH}_{3} \mathrm{CN}(1 \mathrm{~mL})$ were sequentially added $\mathrm{NaHCO}_{3}\left(6 \mathrm{mg}, 0.075 \mathrm{mmol}, 1.5\right.$ equiv) and $\mathrm{I}_{2}(14 \mathrm{mg}, 0.055 \mathrm{mmol}$, 1.1 equiv). The resulting mixture was kept stirring at ambient temperature for $4 \mathrm{~h}$. After complete consumption of alcohol $4 \mathbf{e}$, saturated $a q . \mathrm{Na}_{2} \mathrm{~S}_{2} \mathrm{O}_{3}(1 \mathrm{~mL})$ was added and stirred 
for $5 \mathrm{~min}$ at ambient temperature. Then the reaction mixture was extracted with $\mathrm{Et}_{2} \mathrm{O}(3 \times$ $1 \mathrm{~mL}$ ). The combined organic layers were dried over anhydrous sodium sulfate, filtered, and concentrated under reduced pressure. Purification of the crude product $(3: 1 \mathrm{dr}$ based on ${ }^{1} \mathrm{H}$ NMR) was performed by flash column chromatography (gradient elution with hexane and ethyl acetate, 20:1 to $10: 1)$ to give products $\mathbf{1 0 a}(11 \mathrm{mg}, 61 \%$ yield $)$ and $\mathbf{1 0 b}$ (4 mg, 22\% yield) as colorless oil.

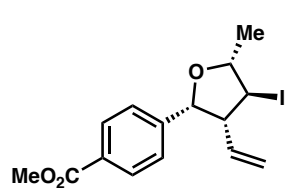

Methyl-4-((2R,3R,4S,5R)-4-iodo-5-methyl-3-vinyltetrahydrofuran2-yl)benzoate (10) Colorless oil. ${ }^{1} \mathrm{H} \mathrm{NMR}\left(600 \mathrm{MHz}, \mathrm{CDCl}_{3}\right) \delta 7.99$ $(\mathrm{d}, J=8.2 \mathrm{~Hz}, 2 \mathrm{H}), 7.28(\mathrm{~d}, J=8.2 \mathrm{~Hz}, 2 \mathrm{H}), 5.22(\mathrm{~d}, J=9.0 \mathrm{~Hz}, 1 \mathrm{H})$, $5.09(\mathrm{~d}, J=16.8 \mathrm{~Hz}, 1 \mathrm{H}), 4.98(\mathrm{~d}, J=10.0 \mathrm{~Hz}, 1 \mathrm{H}), 4.84(\mathrm{ddd}, J=$ 16.9, 9.8, $9.8 \mathrm{~Hz}, 1 \mathrm{H}), 4.24-4.33$ (m, 1H), 3.91 (s, 3H), 3.55 (dd, $J=9.7,9.5 \mathrm{~Hz}, 1 \mathrm{H})$, 3.38 (app. q, $J=9.5 \mathrm{~Hz}, 1 \mathrm{H}), 1.55$ (d, $J=6.0 \mathrm{~Hz}, 3 \mathrm{H}) .{ }^{13} \mathrm{C}$ NMR $\left(151 \mathrm{MHz}, \mathrm{CDCl}_{3}\right) \delta$ 167.2, 144.7, 134.5, 129.7, 129.6, 127.0, 119.5, 83.7, 82.1, 60.3, 52.5, 32.4, 17.7. HRMS $\left(\mathrm{ESI}^{+}\right.$): $m / z$ for $\mathrm{C}_{15} \mathrm{H}_{18} \mathrm{O}_{3} \mathrm{I}[\mathrm{M}+\mathrm{H}]^{+}$calcd. 373.0301, found 373.0294.

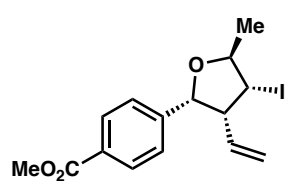

Methyl-4-((2R,3R,4R,5S)-4-iodo-5-methyl-3-vinyltetrahydrofuran2-yl)benzoate (10b) Colorless oil. ${ }^{1} \mathrm{H}$ NMR (600 MHz, $\left.\mathrm{CDCl}_{3}\right) \delta 7.98$ $(\mathrm{d}, J=8.2 \mathrm{~Hz}, 2 \mathrm{H}), 7.30(\mathrm{~d}, J=8.2 \mathrm{~Hz}, 2 \mathrm{H}), 5.35(\mathrm{~d}, J=4.9 \mathrm{~Hz}, 1 \mathrm{H})$, $5.32(\mathrm{ddd}, \mathrm{J}=16.9,10.4,10.4 \mathrm{~Hz}, 1 \mathrm{H}), 4.91(\mathrm{~d}, J=10.1 \mathrm{~Hz}, 1 \mathrm{H}), 4.78$ $(\mathrm{d}, J=16.9 \mathrm{~Hz}, 1 \mathrm{H}), 4.47(\mathrm{dq}, J=12.2,6.1 \mathrm{~Hz}, 1 \mathrm{H}), 4.20(\mathrm{dd}, J=9.5,6.0 \mathrm{~Hz}, 1 \mathrm{H}), 3.90$ (s, 3H), 3.17 (ddd, $J=10.8,5.6,5.3 \mathrm{~Hz}, 1 \mathrm{H}), 1.48(\mathrm{~d}, J=6.0 \mathrm{~Hz}, 3 \mathrm{H}) .{ }^{13} \mathrm{C}$ NMR $(151$ $\left.\mathrm{MHz}_{,} \mathrm{CDCl}_{3}\right) \delta 167.3,145.0,135.4,129.7,129.3,126.2,120.0,82.2,81.2,56.0,52.5$, 33.1, 18.9. HRMS (ESI $): m / z$ for $\mathrm{C}_{15} \mathrm{H}_{18} \mathrm{O}_{3} \mathrm{I}[\mathrm{M}+\mathrm{H}]^{+}$calcd. 373.0301 , found 373.0304 .

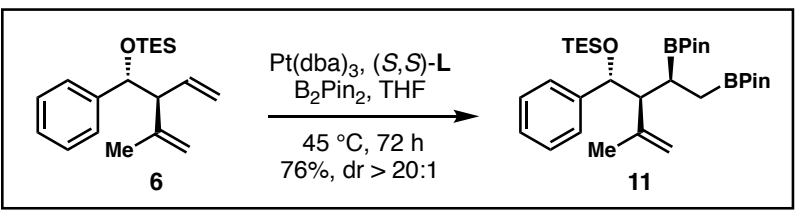

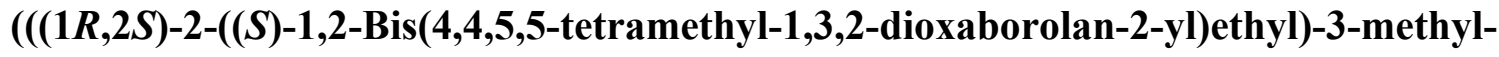
1-phenylbut-3-en-1-yl)oxy)triethylsilane (11) In a glove box, to a reaction vial containing a stirring bar were sequentially added $\operatorname{Pt}(\mathrm{dba})_{3}(3.9 \mathrm{mg}, 0.0043 \mathrm{mmol}, 5$ mol \%), (S,S)-L (4.7 mg, $0.0052 \mathrm{mmol}, 6 \mathrm{~mol} \%), \mathrm{B}_{2} \mathrm{Pin}_{2}$ (24 mg, $0.095 \mathrm{mmol}, 1.1$ equiv), and THF $(0.5 \mathrm{~mL})$. The reaction vial was removed from glove box and stirred at $80{ }^{\circ} \mathrm{C}$ for $1 \mathrm{~h}$. The reaction was cooled to ambient temperature and compound 6 (26 mg, 0.086 mmol, 1.0 equiv) was added. The mixture was stirred at $45^{\circ} \mathrm{C}$ for $72 \mathrm{~h}$. After complete 
consumption of $\mathbf{6}$, the reaction mixture was cooled to ambient temperature and diluted with $\mathrm{Et}_{2} \mathrm{O}(2 \mathrm{~mL})$. The resulting mixture was filtered through a pad of silica gel and the filtrate was concentrated under reduced pressure. Purification of the crude product was performed by flash column chromatography (gradient elution with hexane and diethyl ether, 100:1 to 10:1) to give product 11 as a colorless oil (31 mg, $76 \%$ yield, $d r>20: 1$ ). $[\alpha]_{\mathrm{D}}{ }^{25}=2.80^{\circ}\left(c 0.50, \mathrm{CHCl}_{3}\right) ;{ }^{1} \mathrm{H} \mathrm{NMR}\left(600 \mathrm{MHz}, \mathrm{CDCl}_{3}\right) \delta 7.18-7.22(\mathrm{~m}, 4 \mathrm{H}), 7.14-$ 7.17 (m, 1H), 4.96 (d, $J=8.6 \mathrm{~Hz}, 1 \mathrm{H}), 4.55$ (s, 1H), 4.33 (s, 1H), 2.42 (dd, $J=7.7,7.7$ $\mathrm{Hz}, 1 \mathrm{H}), 1.76-1.79$ (m, 1H), 1.46 (s, 3H), 1.24 (s, 12H), 1.224 (s, 6H), 1.219 (s, 6H), 0.98 (dd, $J=15.8,11.0 \mathrm{~Hz}, 1 \mathrm{H}), 0.93(\mathrm{dd}, J=15.9,4.8 \mathrm{~Hz}, 1 \mathrm{H}), 0.83(\mathrm{t}, J=7.9 \mathrm{~Hz}, 9 \mathrm{H})$, $0.40-0.53(\mathrm{~m}, 6 \mathrm{H}) .{ }^{13} \mathrm{C}$ NMR $\left(151 \mathrm{MHz}, \mathrm{CDCl}_{3}\right) \delta 146.2,144.5,127.8,127.5,127.0$, $113.9,83.0,76.8,59.6,25.5,25.4,25.2,25.0,24.5,20.3,12.5,7.2,5.2$. HRMS $\left(\mathrm{ESI}^{+}\right)$: $m / z$ for $\mathrm{C}_{31} \mathrm{H}_{54} \mathrm{~B}_{2} \mathrm{O}_{5} \mathrm{SiNa}[\mathrm{M}+\mathrm{Na}]^{+}$calcd. 579.3824, found 579.3826.

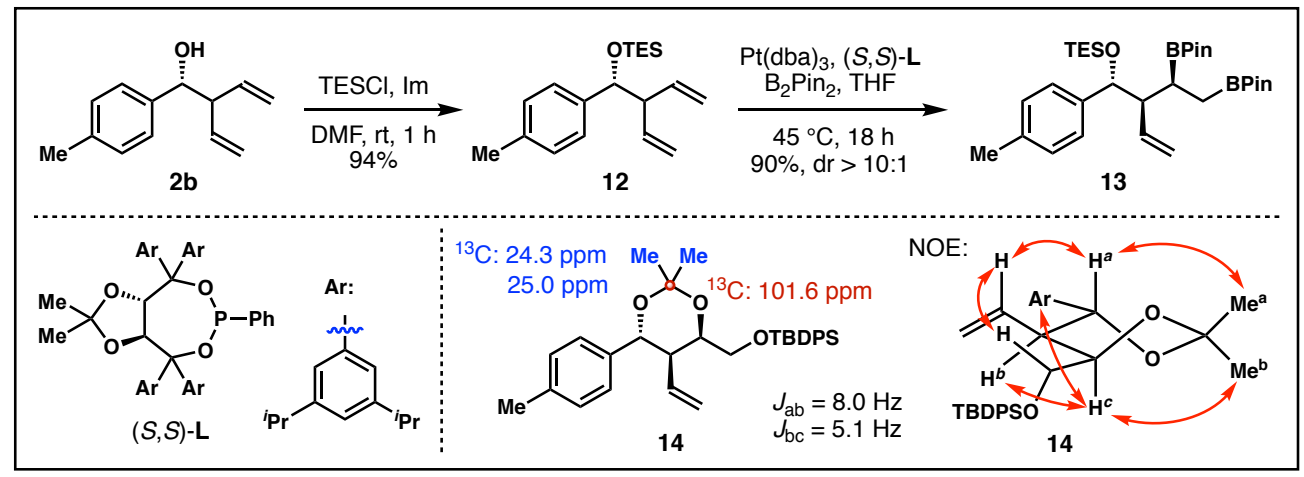

$(((1 R, 2 S)-2-((S)-1,2-B i s(4,4,5,5-$ tetramethyl-1,3,2-dioxaborolan-2-yl)ethyl)-1-(p-tolyl) but-3-en-1-yl)oxy)triethylsilane In a reaction vial, $2 \mathbf{b}$ (17 $\mathrm{mg}, 0.09 \mathrm{mmol}, 1.0$ equiv), imidazole (12 mg, $0.18 \mathrm{mmol}, 2.0$ equiv) and TESCl (27 mg, $0.18 \mathrm{mmol}, 2.0$ equiv) were dissolved in DMF $(1 \mathrm{~mL})$. The reaction mixture was stirred at ambient temperature. After complete consumption of alcohol $\mathbf{2} \mathbf{b}$, the reaction was diluted with water $(5 \mathrm{~mL})$, and the resulting solution was extracted with diethyl ether $(3 \times 3 \mathrm{~mL})$. The combined organic layers were dried over anhydrous sodium sulfate, filtered, and concentrated under reduced pressure. Purification of the crude product was performed by flash column chromatography (gradient elution with hexane and ethyl acetate, 100:1 to 20:1) to give product $12\left(26 \mathrm{mg}, 94 \%\right.$ yield) as colorless oil. ${ }^{1} \mathrm{H}$ NMR $\left(600 \mathrm{MHz}, \mathrm{CDCl}_{3}\right) \delta 7.14(\mathrm{~d}, J$ $=8.1 \mathrm{~Hz}, 2 \mathrm{H}), 7.08(\mathrm{~d}, J=8.0 \mathrm{~Hz}, 2 \mathrm{H}), 5.84(\mathrm{ddd}, J=17.6,10.4,7.7 \mathrm{~Hz}, 1 \mathrm{H}), 5.78$ (ddd, $J=17.4,10.4,7.3 \mathrm{~Hz}, 1 \mathrm{H}), 5.06(\mathrm{~d}, J=10.3 \mathrm{~Hz}, 1 \mathrm{H}), 4.92-5.00(\mathrm{~m}, 3 \mathrm{H}), 4.60$ (d, $J=$ $5.8 \mathrm{~Hz}, 1 \mathrm{H}$ ), 2.99 (ddd, $J=7.2,7.2,6.6 \mathrm{~Hz}, 1 \mathrm{H}), 2.32$ (s, 3H), 0.85 (t, $J=8.0 \mathrm{~Hz}, 9 \mathrm{H}$ ), $0.44-0.54(\mathrm{~m}, 6 \mathrm{H}) .{ }^{13} \mathrm{C} \mathrm{NMR}\left(151 \mathrm{MHz}, \mathrm{CDCl}_{3}\right) \delta 140.5,138.4,137.8,136.8,128.6$, 
$127.0,116.7,116.3,77.9,57.2,21.5,7.2,5.1$.

In a glove box, to a reaction vial containing a stirring bar were sequentially added $\mathrm{Pt}(\mathrm{dba})_{3}(3.9 \mathrm{mg}, 0.0043 \mathrm{mmol}, 5 \mathrm{~mol} \%),(S, S)-\mathbf{L}(4.7 \mathrm{mg}, 0.0052 \mathrm{mmol}, 6 \mathrm{~mol} \%)$, $\mathrm{B}_{2} \mathrm{Pin}_{2}$ (24 mg, $0.095 \mathrm{mmol}, 1.1$ equiv), and THF $(0.5 \mathrm{~mL})$. The reaction vial was removed from glove box and stirred at $80^{\circ} \mathrm{C}$ for $1 \mathrm{~h}$. The reaction was cooled to ambient temperature and compound 12 (26 $\mathrm{mg}, 0.086 \mathrm{mmol}, 1.0$ equiv) was added. The mixture was stirred at $45^{\circ} \mathrm{C}$ for $18 \mathrm{~h}$. After complete consumption of $\mathbf{1 2}$, the reaction mixture was cooled to ambient temperature and diluted with $\mathrm{Et}_{2} \mathrm{O}(2 \mathrm{~mL})$. The resulting mixture was filtered through a pad of silica gel and the filtrate was concentrated under reduced pressure. Purification of the crude product was performed by flash chromatography (gradient elution with hexane and diethyl ether, 100:1 to 10:1) to give product 13 as a colorless oil (43 mg, 90\% yield, $d r>10: 1$ ). The 1,3-anti relative configuration of $\mathbf{1 3}$ was assigned by NOE, ${ }^{1} \mathrm{H}$ and ${ }^{13} \mathrm{C}$ NMR studies of the corresponding acetonide derivative $14 .{ }^{6}$ $[\alpha]_{\mathrm{D}}{ }^{25}=19.4^{\circ}\left(c 1.30, \mathrm{CHCl}_{3}\right) ;{ }^{1} \mathrm{H}$ NMR $\left(400 \mathrm{MHz}, \mathrm{CDCl}_{3}\right) \delta 7.08(\mathrm{~d}, J=7.9 \mathrm{~Hz}, 2 \mathrm{H})$, $7.02(\mathrm{~d}, J=7.8 \mathrm{~Hz}, 2 \mathrm{H}), 5.39$ (ddd, $J=17.1,10.1,10.0 \mathrm{~Hz}, 1 \mathrm{H}), 4.79$ (d, $J=8.3 \mathrm{~Hz}, 1 \mathrm{H})$, $4.74(\mathrm{dd}, J=10.2,1.9 \mathrm{~Hz}, 1 \mathrm{H}), 4.65(\mathrm{dd}, J=17.0,1.6 \mathrm{~Hz}, 1 \mathrm{H}), 2.29$ (s, 3H), 2.25 (ddd, $J$ $=9.2,9.2,4.7 \mathrm{~Hz}, 1 \mathrm{H}), 1.80(\mathrm{ddd}, J=10.1,5.3,5.2 \mathrm{~Hz}, 1 \mathrm{H}), 1.23$ (s, 24H), 0.98 (dd, $J=$ $15.8,10.2 \mathrm{~Hz}, 1 \mathrm{H}), 0.85$ (t, $J=7.9 \mathrm{~Hz}, 9 \mathrm{H}), 0.74$ (dd, $J=15.8,5.7 \mathrm{~Hz}, 1 \mathrm{H}), 0.40-0.56$ $(\mathrm{m}, 6 \mathrm{H}) .{ }^{13} \mathrm{C} \mathrm{NMR}\left(151 \mathrm{MHz}, \mathrm{CDCl}_{3}\right) \delta 141.7,138.0,136.4,128.5,127.3,117.0,83.03$, 83.01, 76.4, 58.9, 25.39, 25.35, 25.2, 25.0, 21.6, 19.3, 12.0, 7.2, 5.2. HRMS (ESI $\left.{ }^{+}\right): m / z$ for $\mathrm{C}_{31} \mathrm{H}_{54} \mathrm{~B}_{2} \mathrm{O}_{5} \mathrm{SiNa}[\mathrm{M}+\mathrm{Na}]^{+}$calcd. 579.3824, found 579.3822.

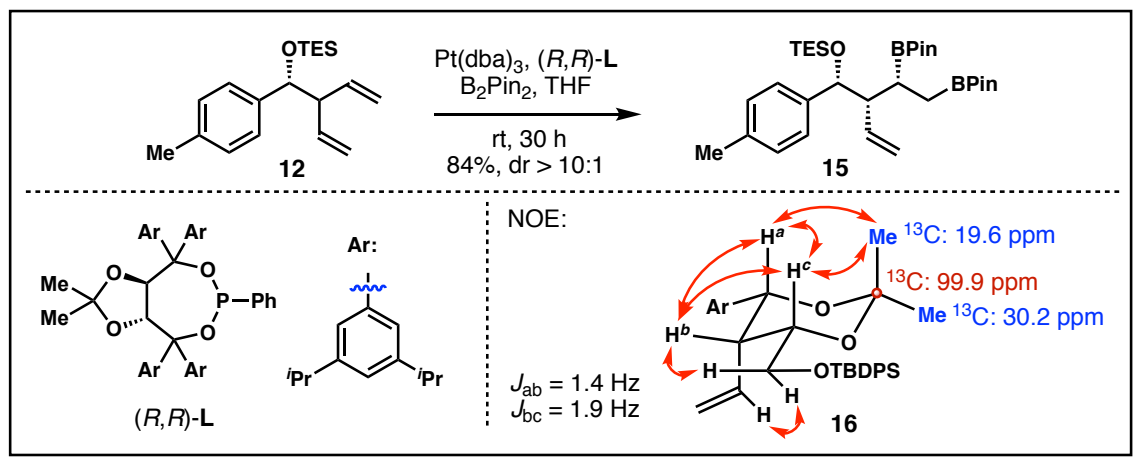

$(((1 R, 2 R)-2-((R)-1,2-B i s(4,4,5,5-t e t r a m e t h y l-1,3,2-d i o x a b o r o l a n-2-y l) e t h y l)-1-(p$-tolyl )but-3-en-1-yl)oxy)triethylsilane (15) In a glove box, to a reaction vial containing a stirring bar were sequentially added $\mathrm{Pt}(\mathrm{dba})_{3}(3.3 \mathrm{mg}, 0.0036 \mathrm{mmol}, 5 \mathrm{~mol} \%),(R, R)-\mathbf{L}$ (4.0 mg, $0.0044 \mathrm{mmol}, 6 \mathrm{~mol} \%$ ), $\mathrm{B}_{2} \mathrm{Pin}_{2}(20 \mathrm{mg}, 0.08 \mathrm{mmol}, 1.1$ equiv), and THF (0.5 $\mathrm{mL}$ ). The reaction vial was removed from glove box and stirred at $80{ }^{\circ} \mathrm{C}$ for $1 \mathrm{~h}$. The 
reaction was cooled to ambient temperature and compound 12 (22 $\mathrm{mg}, 0.073 \mathrm{mmol}, 1.0$ equiv) was added. The mixture was stirred at ambient temperature for $30 \mathrm{~h}$. After complete consumption of $\mathbf{1 2}$, the reaction mixture was cooled to ambient temperature and diluted with $\mathrm{Et}_{2} \mathrm{O}(2 \mathrm{~mL})$. The resulting mixture was filtered through a pad of silica gel and the filtrate was concentrated under reduced pressure. Purification of the crude product was performed by flash chromatography (gradient elution with hexane and diethyl ether, 100:1 to 10:1) to give product 15 as a colorless oil (34 $\mathrm{mg}, 84 \%$ yield, $d r>$ 10:1). The 1,3-syn relative configuration of 15 was assigned by $\mathrm{NOE},{ }^{1} \mathrm{H}$ and ${ }^{13} \mathrm{C} \mathrm{NMR}$ studies of the corresponding acetonide derivative $16 .{ }^{6}[\alpha]_{\mathrm{D}}{ }^{25}=15.3^{\circ}\left(c 0.80, \mathrm{CHCl}_{3}\right) ;{ }^{1} \mathrm{H}$ NMR (400 MHz, $\left.\mathrm{CDCl}_{3}\right) \delta 7.18(\mathrm{~d}, J=7.8 \mathrm{~Hz}, 2 \mathrm{H}), 7.04$ (d, $\left.J=7.7 \mathrm{~Hz}, 2 \mathrm{H}\right), 5.75$ (ddd, $J=17.5,10.0,9.9 \mathrm{~Hz}, 1 \mathrm{H}), 4.96(\mathrm{dd}, J=10.0,0.7 \mathrm{~Hz}, 1 \mathrm{H}), 4.86(\mathrm{~d}, J=9.7 \mathrm{~Hz}, 1 \mathrm{H}), 4.84$ $(\mathrm{d}, J=17.6 \mathrm{~Hz}, 1 \mathrm{H}), 2.31(\mathrm{~s}, 3 \mathrm{H}), 2.14-2.20(\mathrm{~m}, 1 \mathrm{H}), 1.22(\mathrm{~s}, 12 \mathrm{H}), 1.19(\mathrm{~s}, 6 \mathrm{H}), 1.18$ $(\mathrm{s}, 6 \mathrm{H}), 1.06-1.14(\mathrm{~m}, 1 \mathrm{H}), 0.80-0.91(\mathrm{~m}, 2 \mathrm{H}), 0.83(\mathrm{t}, J=7.9 \mathrm{~Hz}, 9 \mathrm{H}), 0.45$ (q, $J=$ $7.7 \mathrm{~Hz}, 6 \mathrm{H}) .{ }^{13} \mathrm{C} \mathrm{NMR}\left(101 \mathrm{MHz}, \mathrm{CDCl}_{3}\right) \delta 142.3,139.2,136.2,128.5,127.4,117.1$, 83.12, 83.07, 75.2, 57.6, 25.4, 25.3 (2C), 25.1, 21.5, 7.2, 5.3. HRMS $\left(\mathrm{ESI}^{+}\right): \mathrm{m} / \mathrm{z}$ for $\mathrm{C}_{31} \mathrm{H}_{54} \mathrm{~B}_{2} \mathrm{O}_{5} \mathrm{SiNa}[\mathrm{M}+\mathrm{Na}]^{+}$calcd. 579.3824, found 579.3848 .

\section{Assignment of the absolute configuration using Mosher ester analysis: ${ }^{5}$}

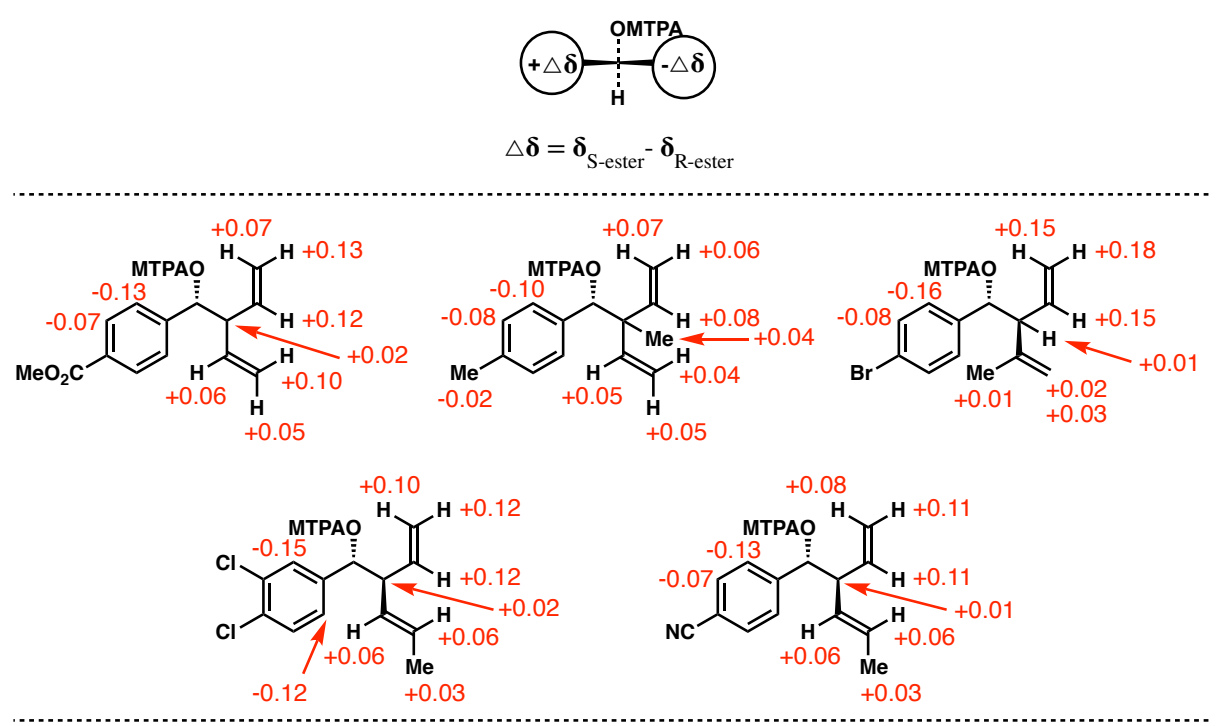




\section{Reference:}

1. Coombs, J. R.; Zhang, L.; Morken, J. P. Org. Lett. 2015, 17, 1708.

2. (a) Herberich, G. E.; Schmidt, B.; Englert, U. Organometallics 1995, 14, 471. (b) Schlosser, M.; Zellner, A.; Leroux, F. Synthesis 2001, 1830.

3. (a) Vaultier, M.; Truchet, F.; Carboni, B.; Hoffmann, R. W.; Denne, I. Tetrahedron Lett. 1987, 28, 4169. (b) Tucker, C. E.; Davidson, J.; Knochel, P. J. Org. Chem. 1992, 57, 3482. (c) Pereira, S.; Srebnik, M. Organometallics 1995, 14 , 3127. (d) Gunawan, C.; Rizzacasa, M. A. Org. Lett. 2010, 12, 1388. (e) Cannillo, A.; Norsikian, S.; Retailleau, P.; Dau, M.-E. T. H.; Iorga, B. I.; Beau, J.-M. Chem. - Eur. J. 2013, 19, 9127.

4. Selander, N.; Kipke, A.; Sebelius, S.; Szabó, k. J. J. Am. Chem. Soc. 2007, 129, 13723.

5. (a) Dale, J. A.; Mosher, H. S. J. Am. Chem. Soc. 1973, 95, 512. (b) Ohtani, I.; Kusumi, T.; Kashman, Y.; Kakisawa, H. J. Am. Chem. Soc. 1991, 113, 4092. (c) Hoye, T. R.; Jeffrey; C. S.; Shao, F. Nat. Protoc. 2007, 2, 2451.

6. (a) Rychnovsky, S. D.; Skalitzky, D. J. Tetrahedron Lett. 1990, 31, 945. (b) Evans, D. A.; Rieger, D. L.; Gage, J. R. Tetrahedron Lett. 1990, 31, 7099. (c) Rychnovsky, S. D.; Rogers, B. N.; Richardson, T. I. Acc. Chem. Res. 1998, 3119. 

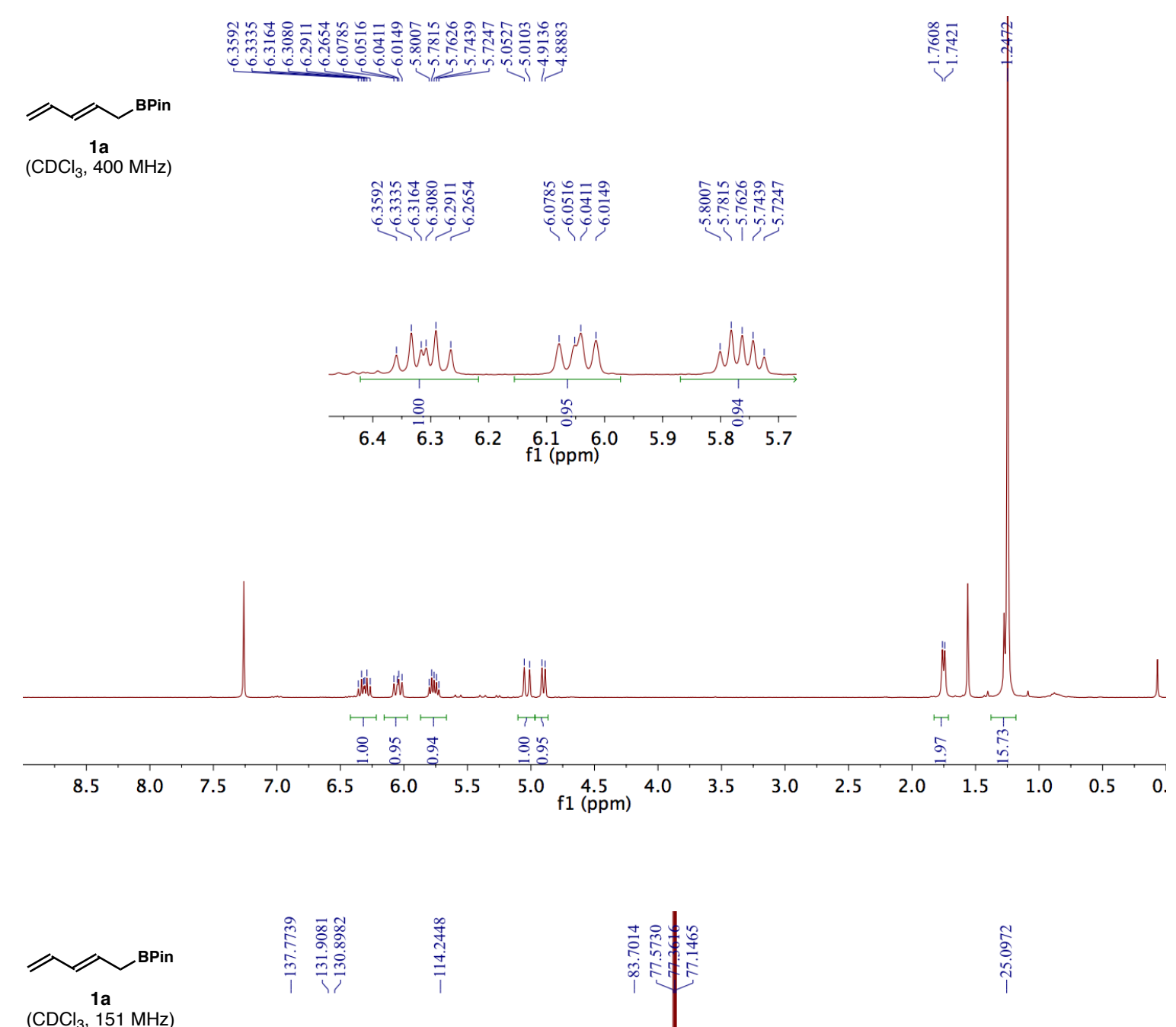

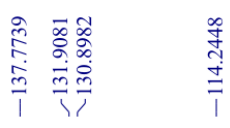

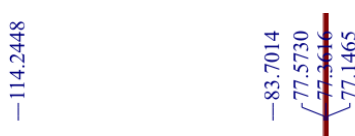

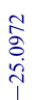
$\left(\mathrm{CDCl}_{3}, 151 \mathrm{MHz}\right)$

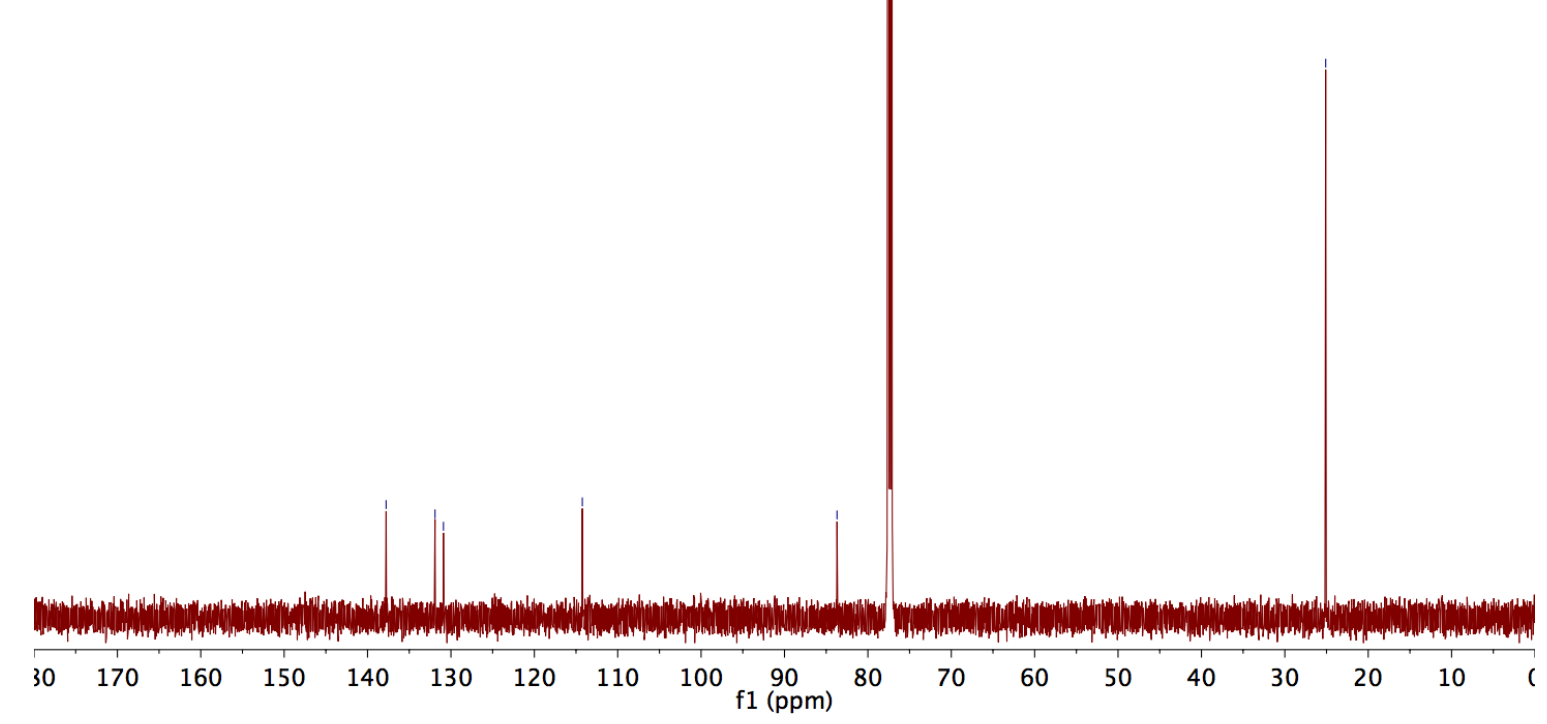



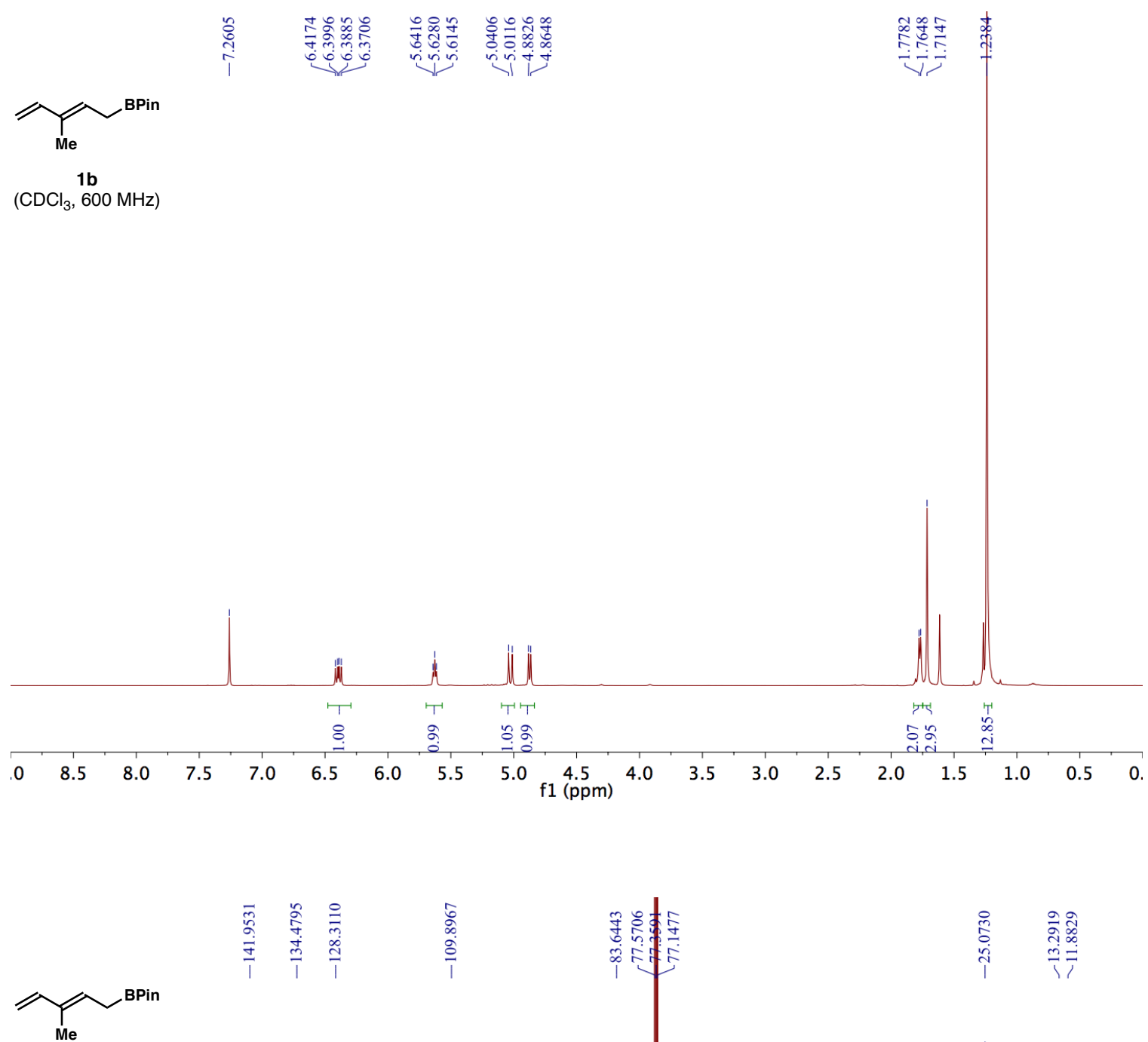

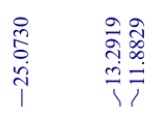

$\left(\mathrm{CDCl}_{3}, 151 \mathrm{MHz}\right)$

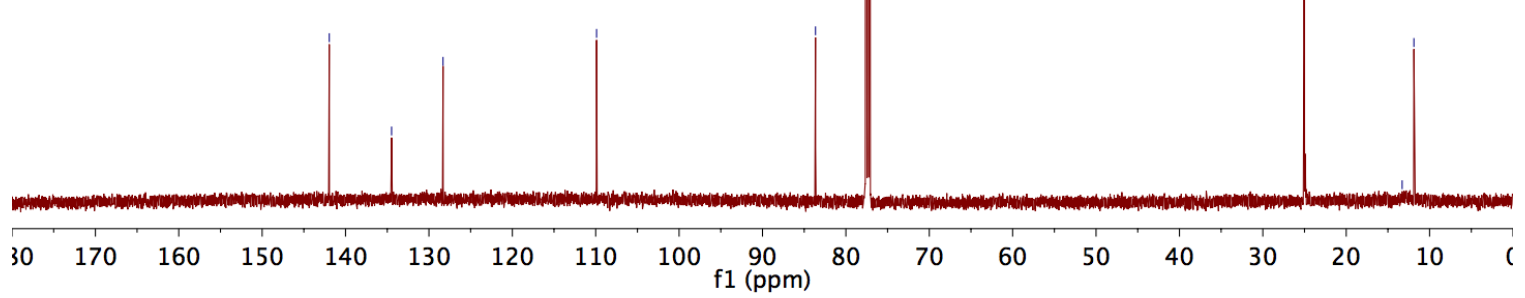

SI-33 

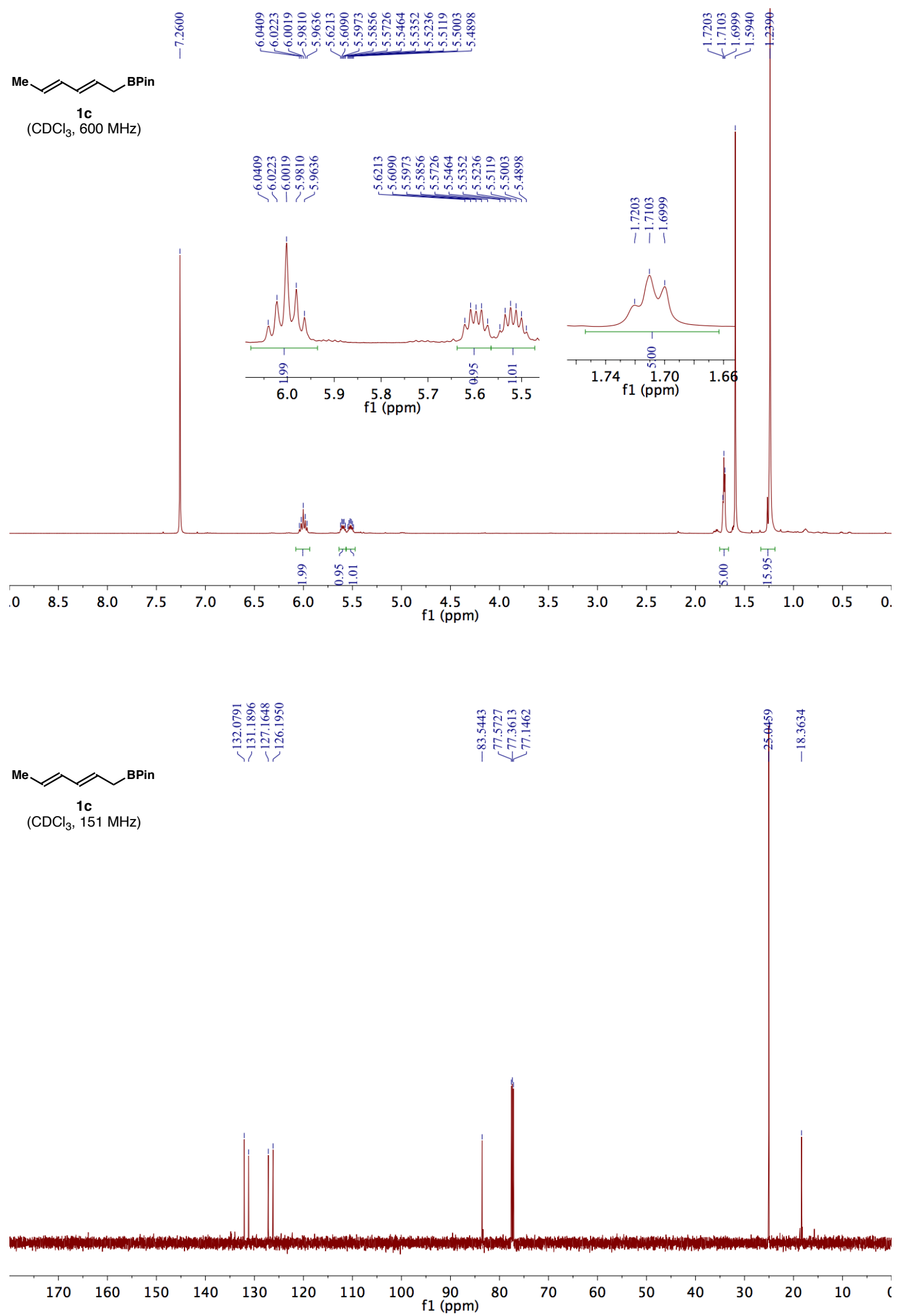

SI-34 

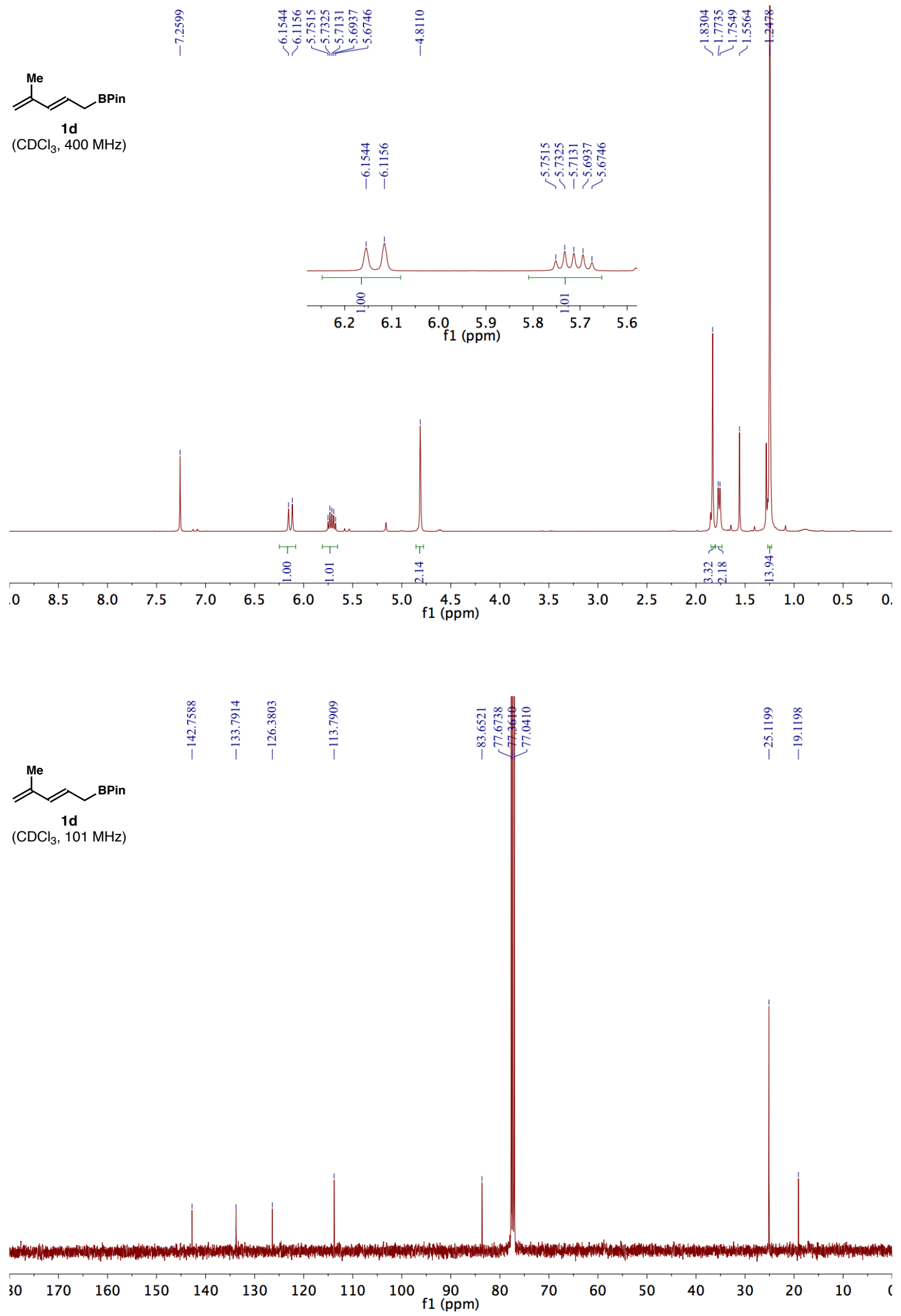

SI-35 

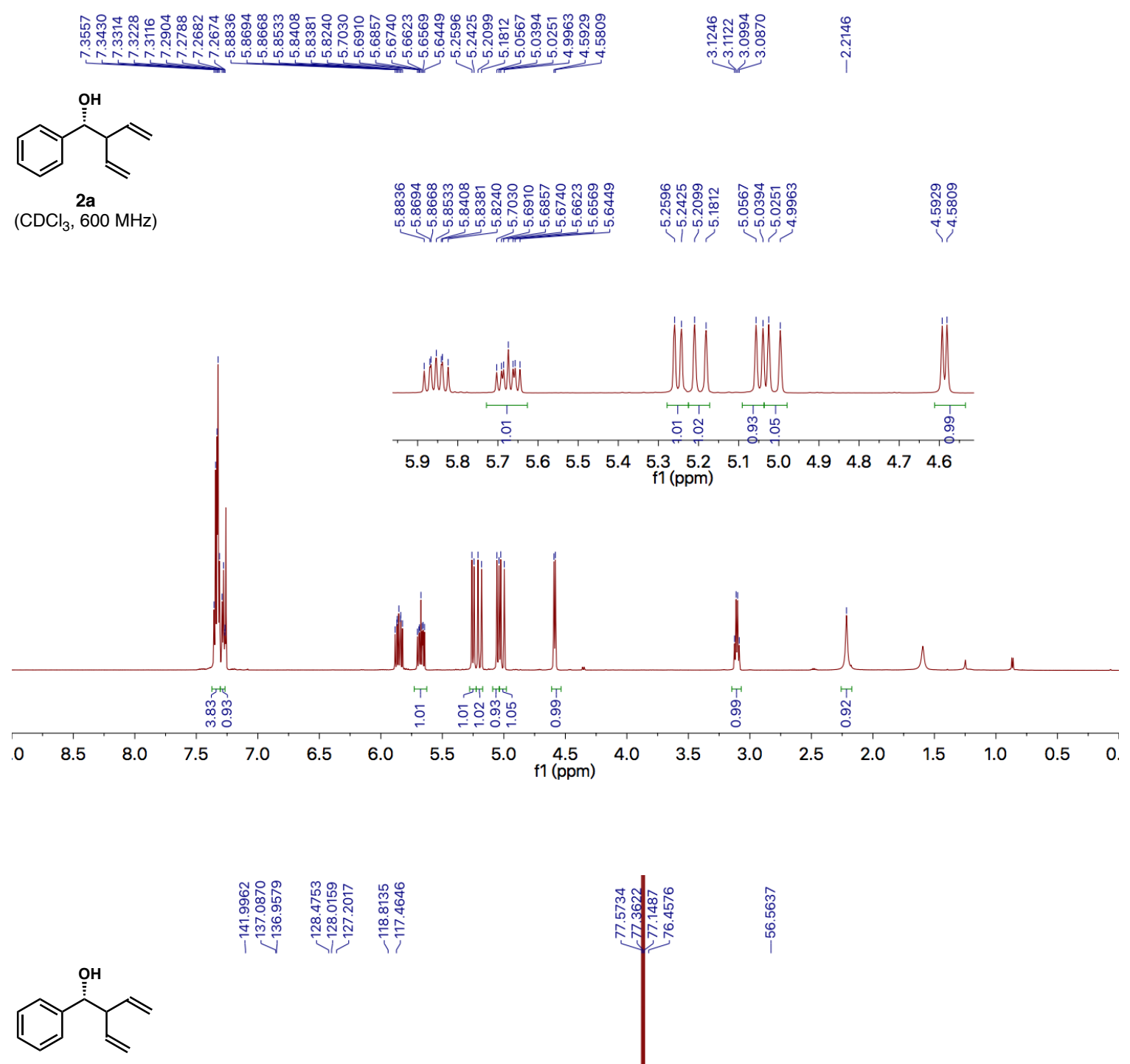

$\left(\mathrm{CDCl}_{3}, 151 \mathrm{MHz}\right)$

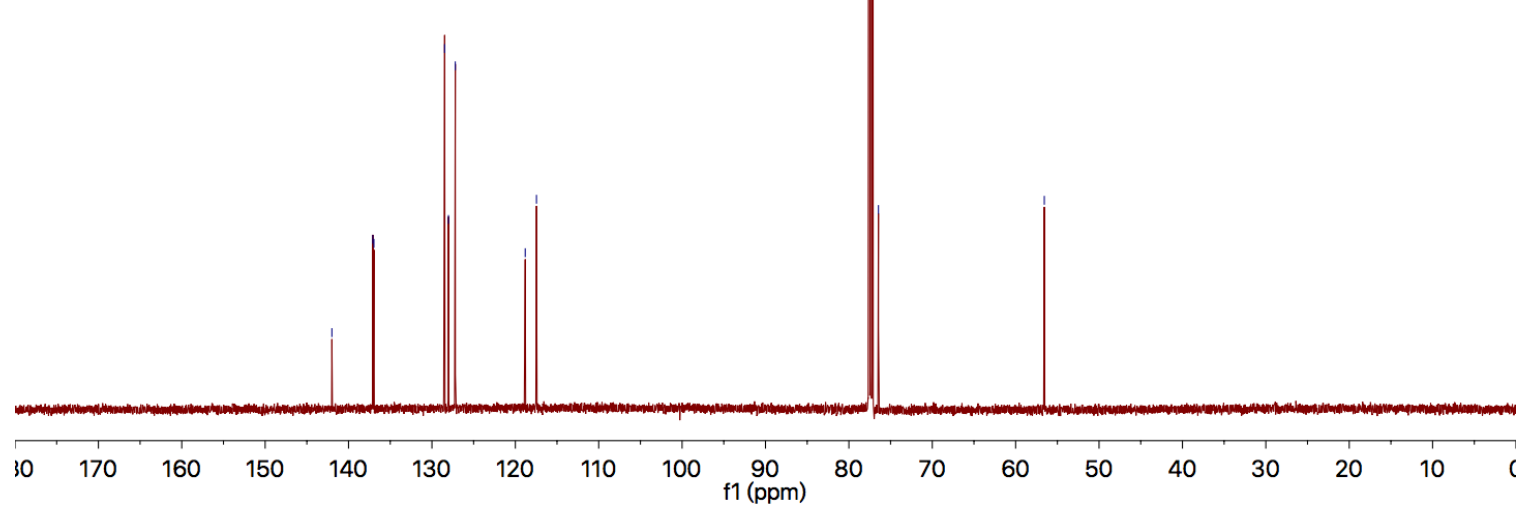



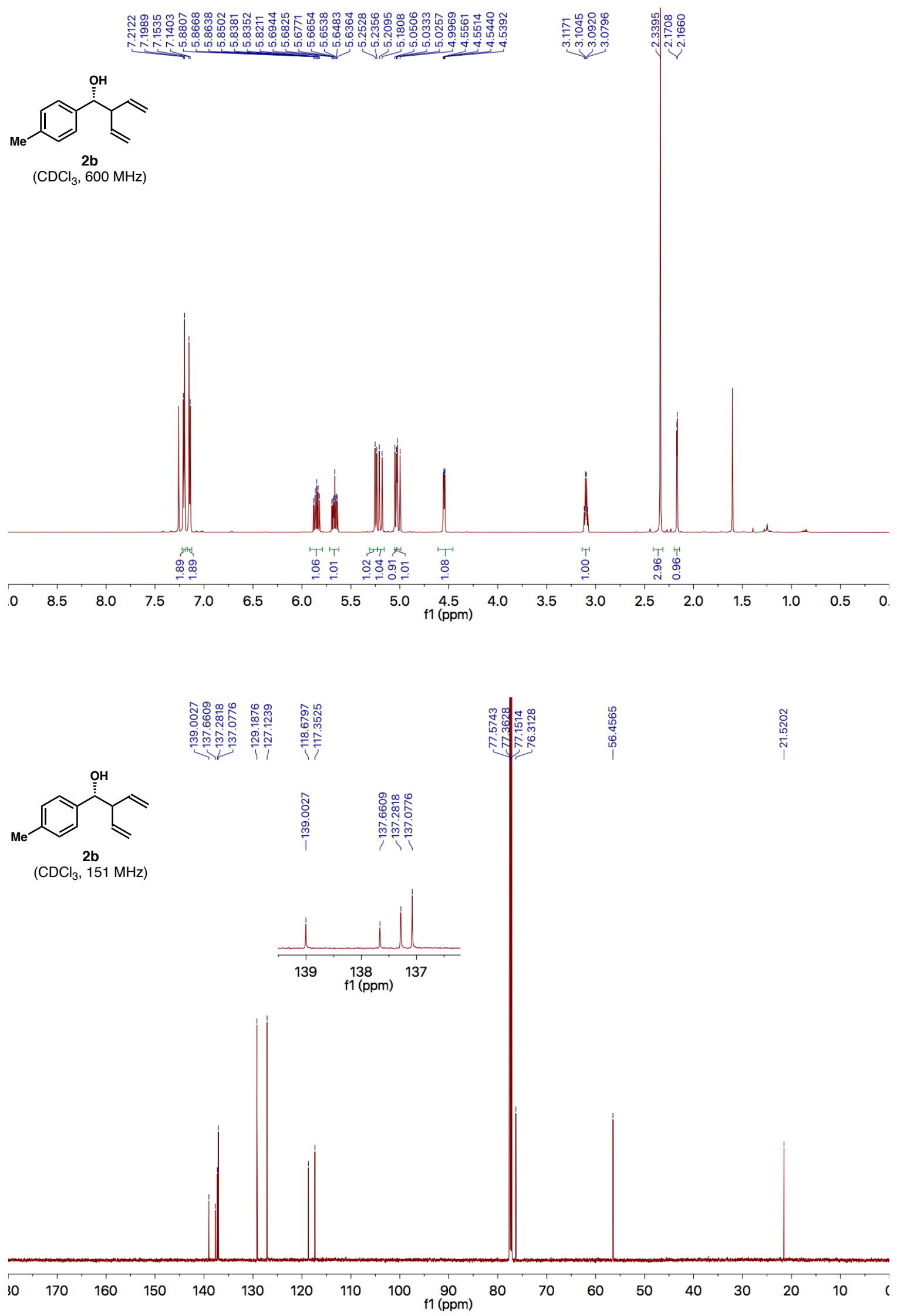

SI-37 

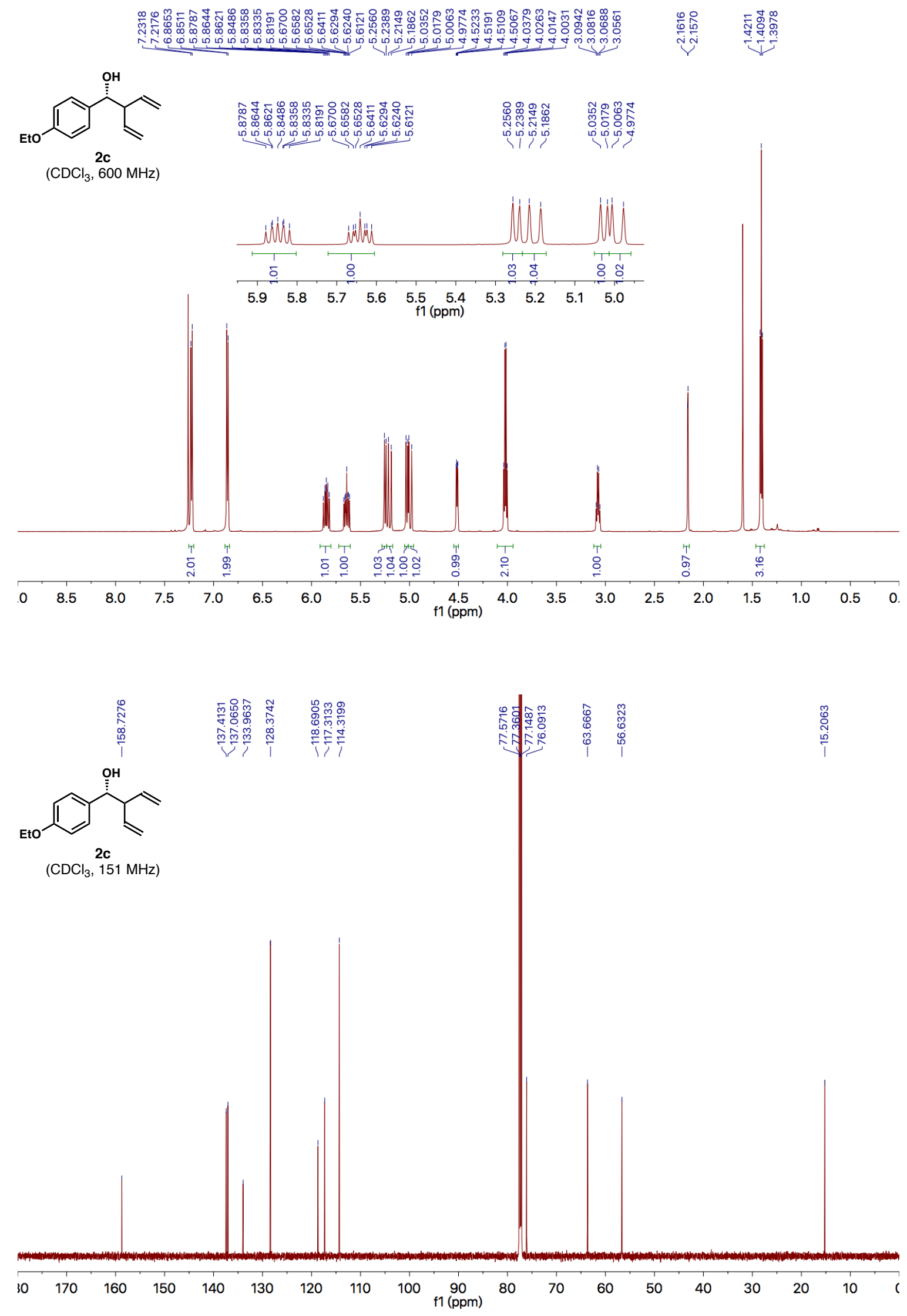

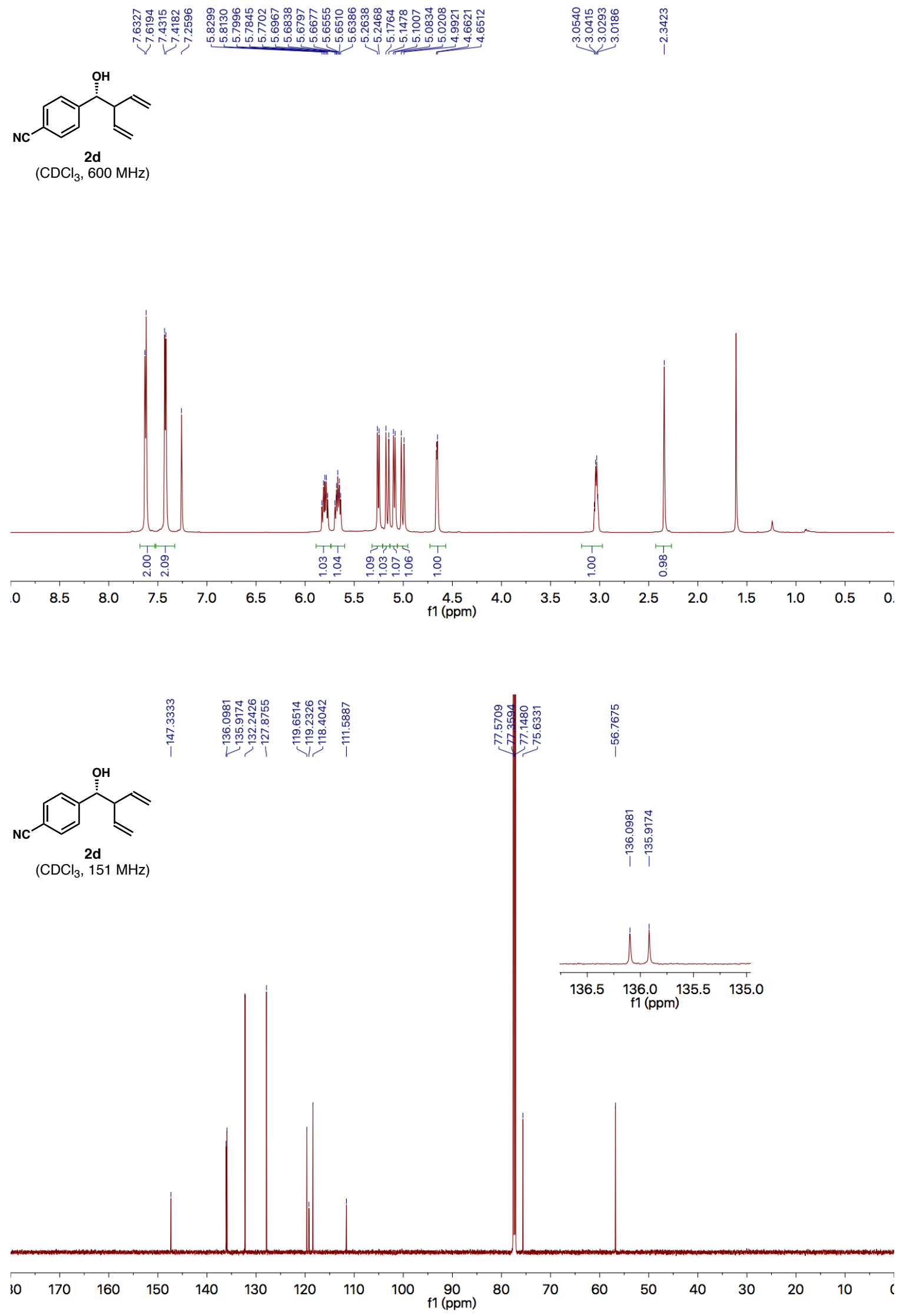

SI-39 

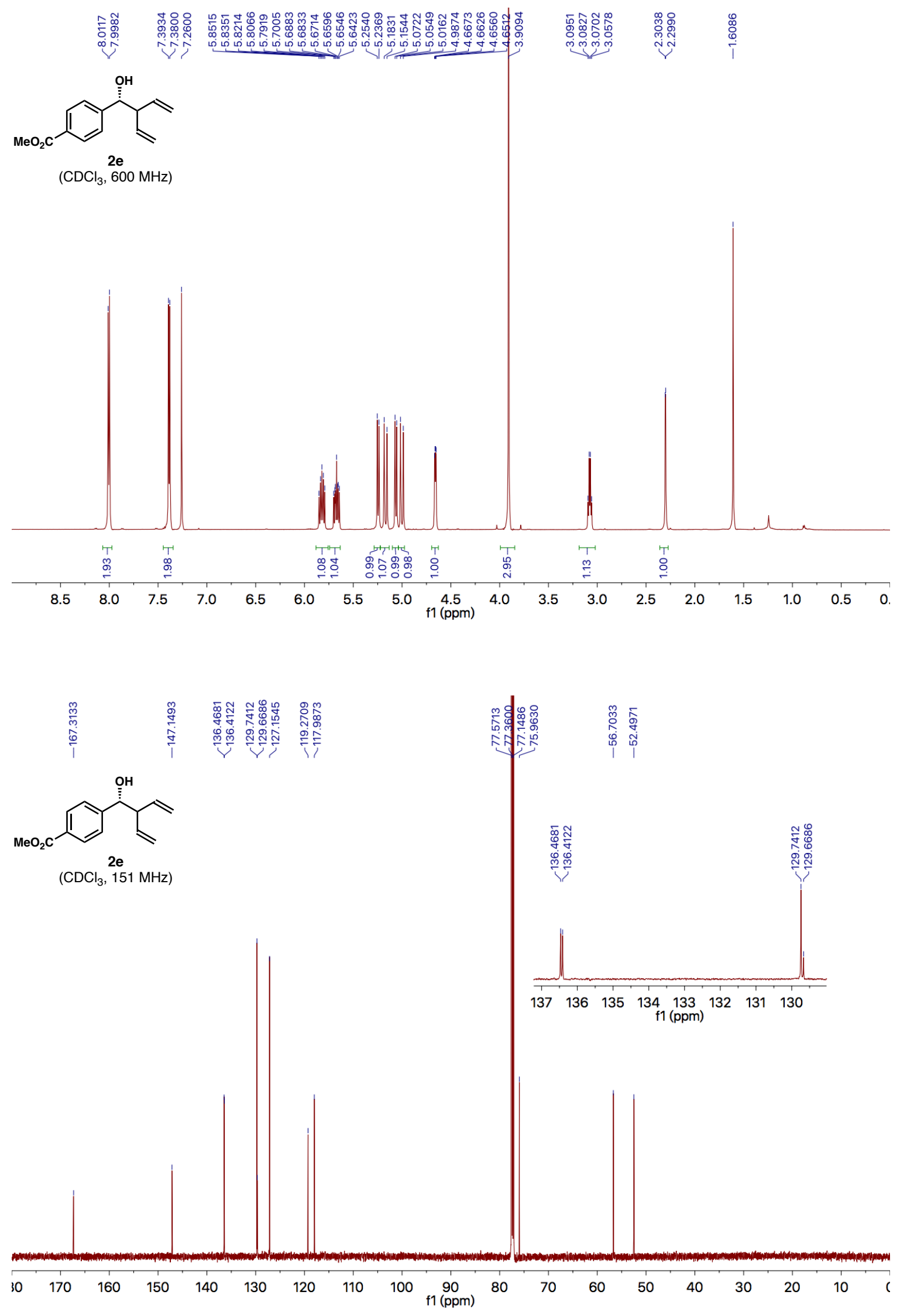

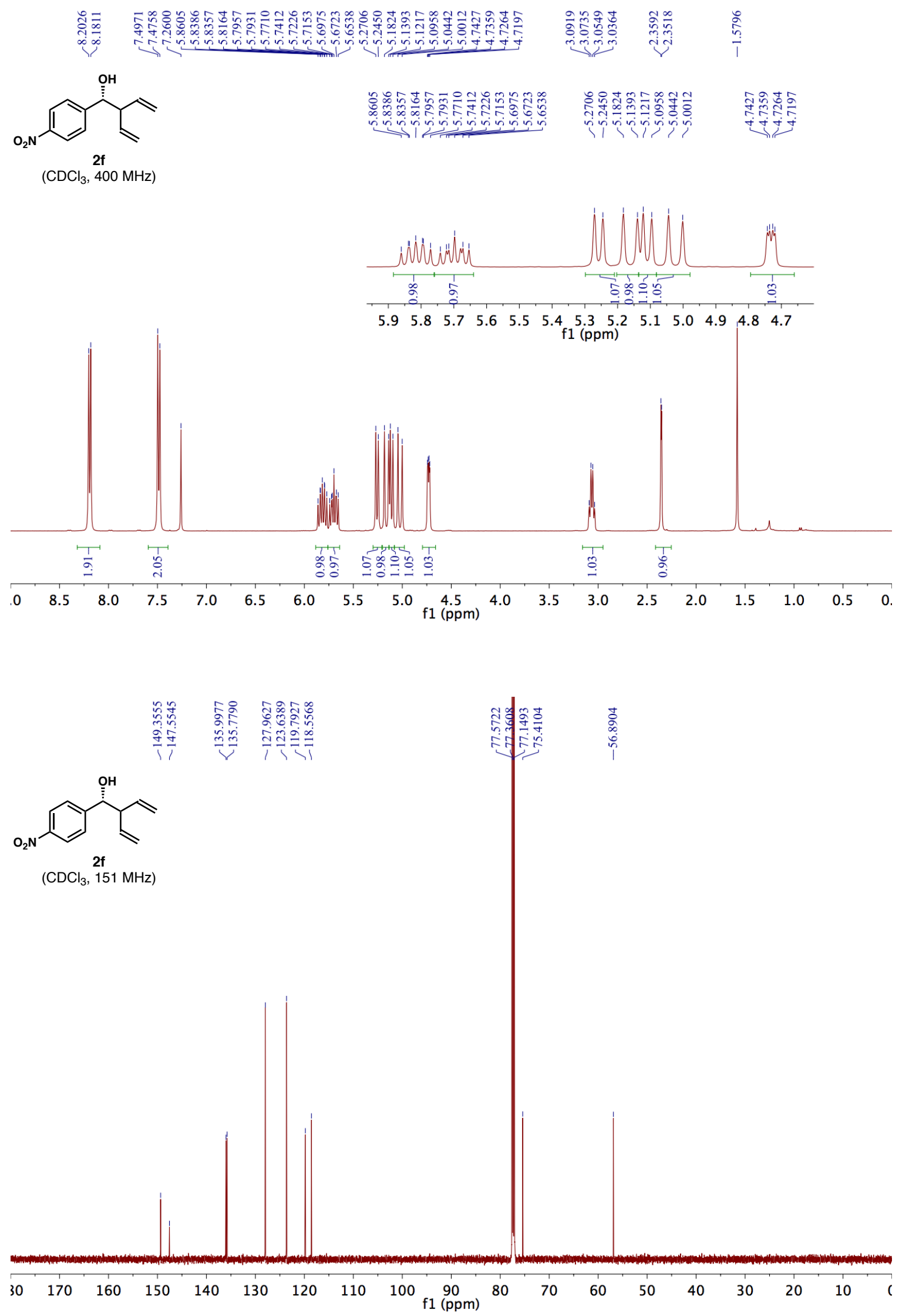

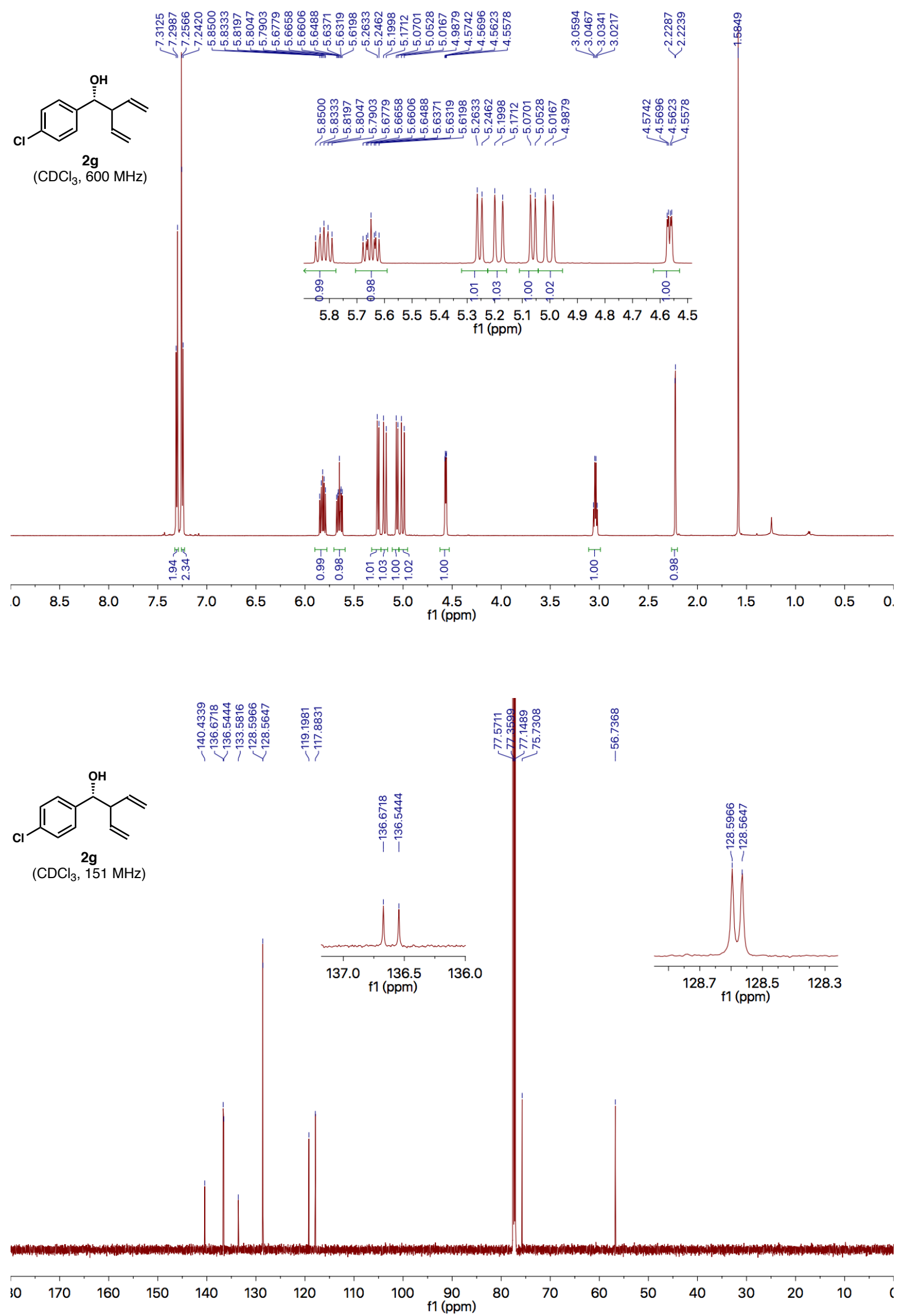

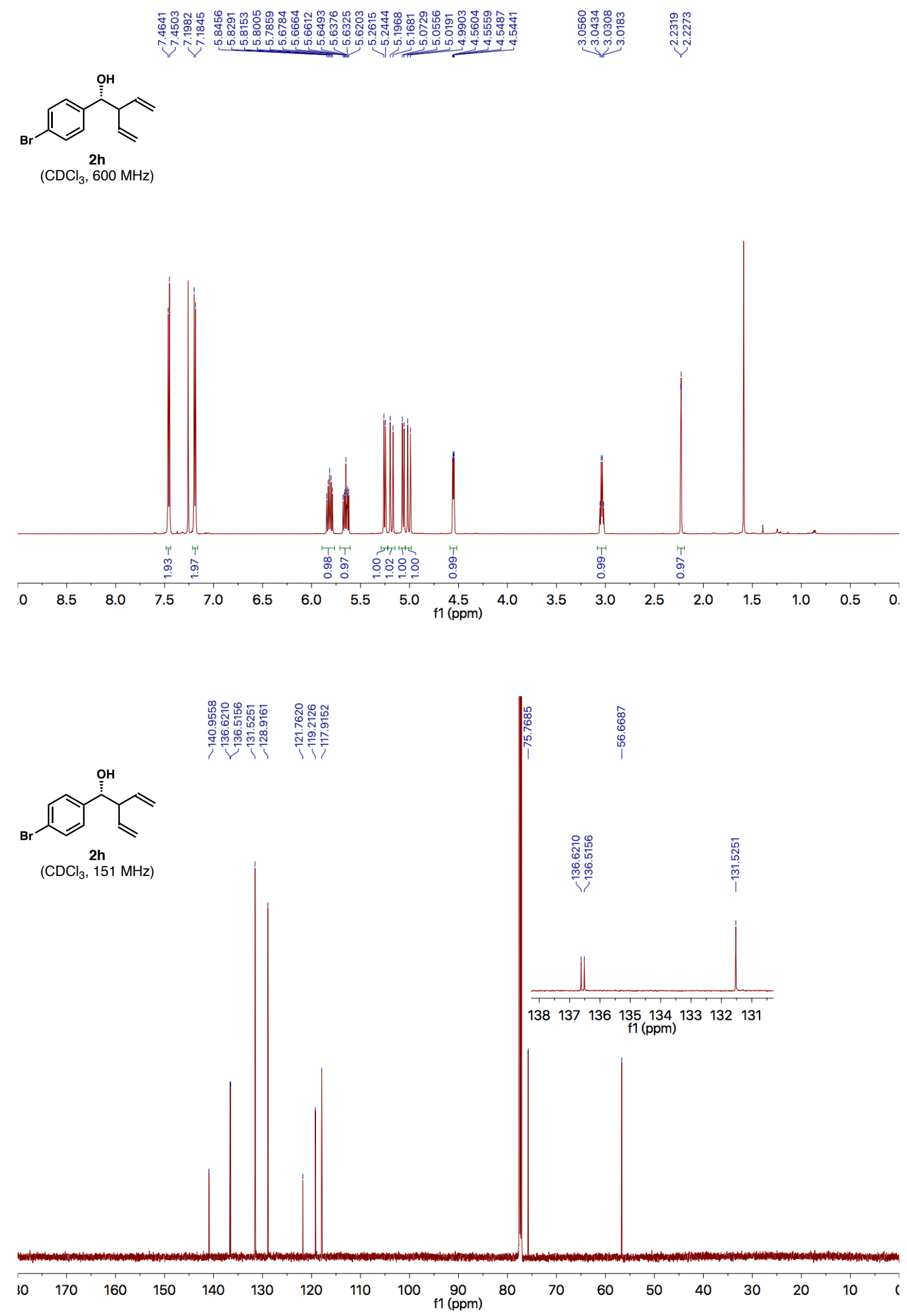

SI-43 

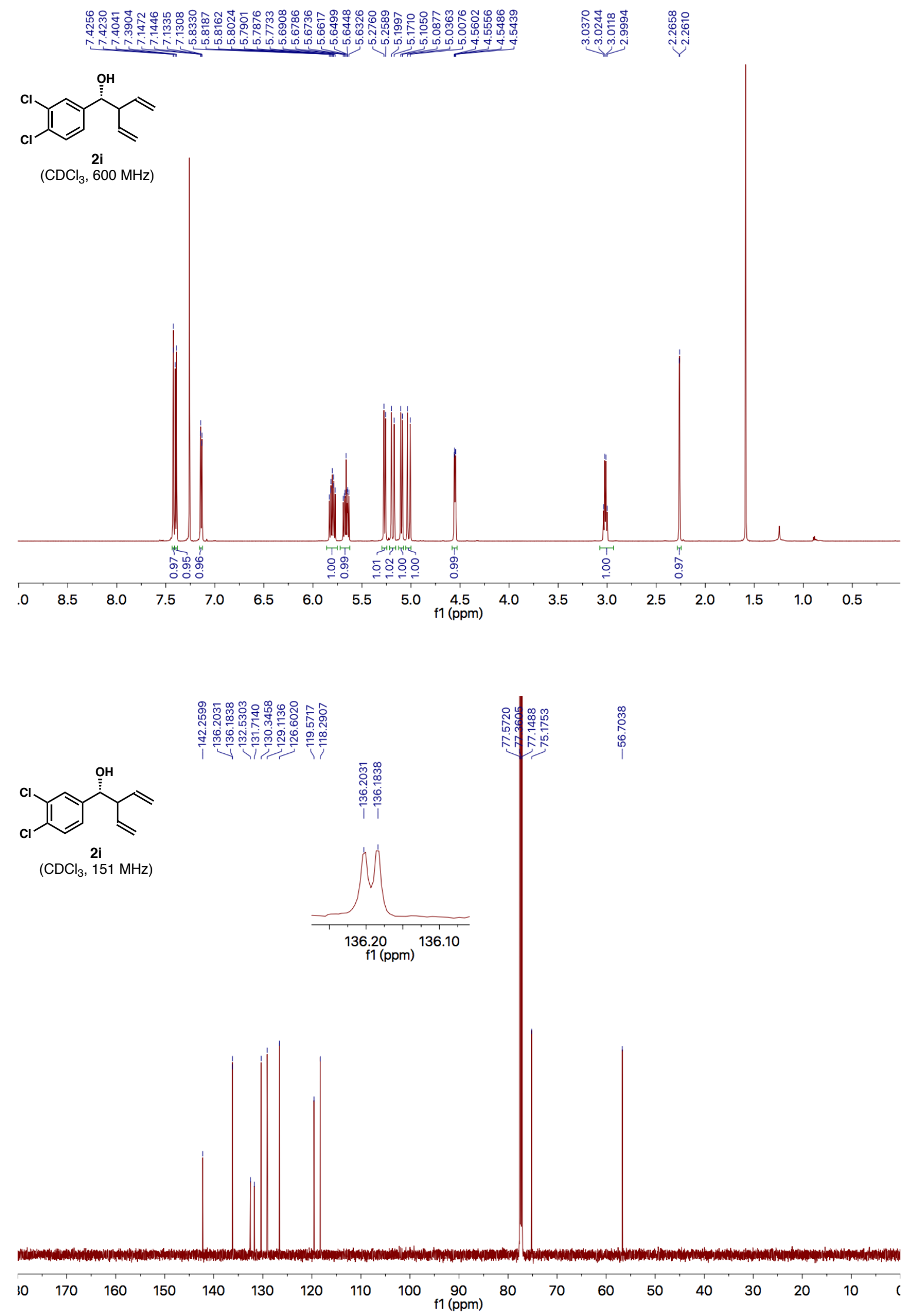

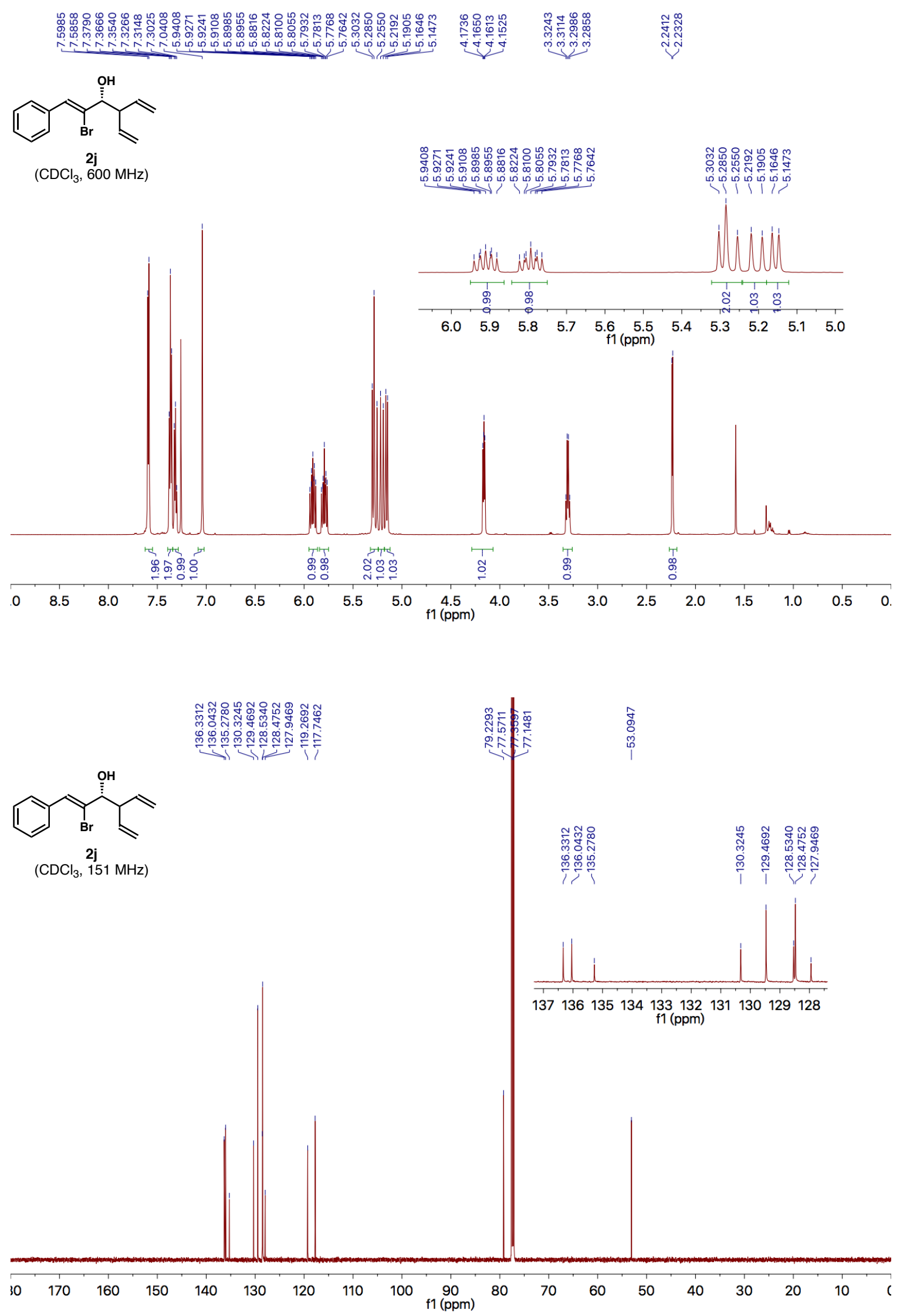

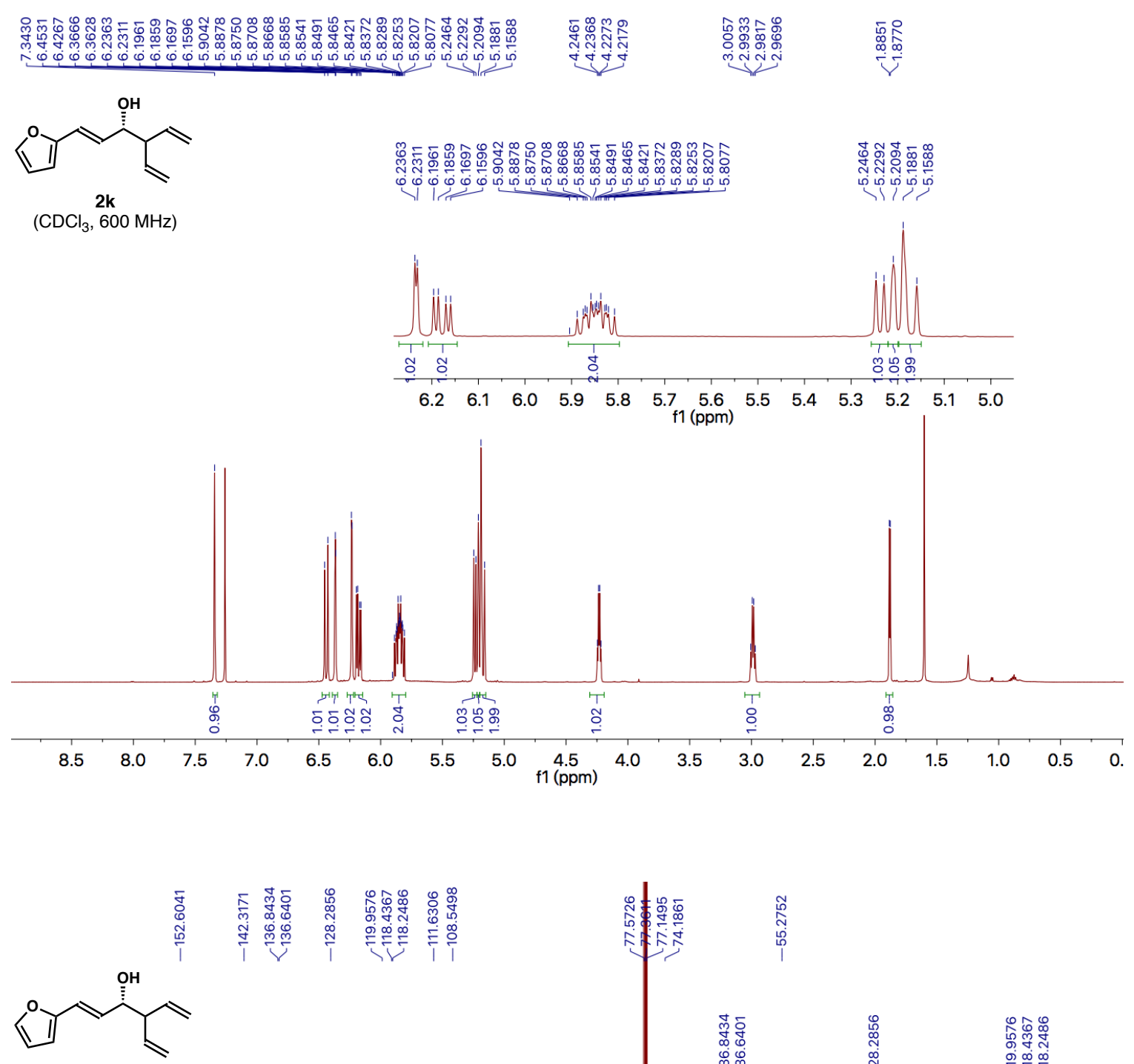

$\stackrel{2 \mathbf{2 k}}{\left(\mathrm{CDCl}_{3}, 151 \mathrm{MHz}\right)}$

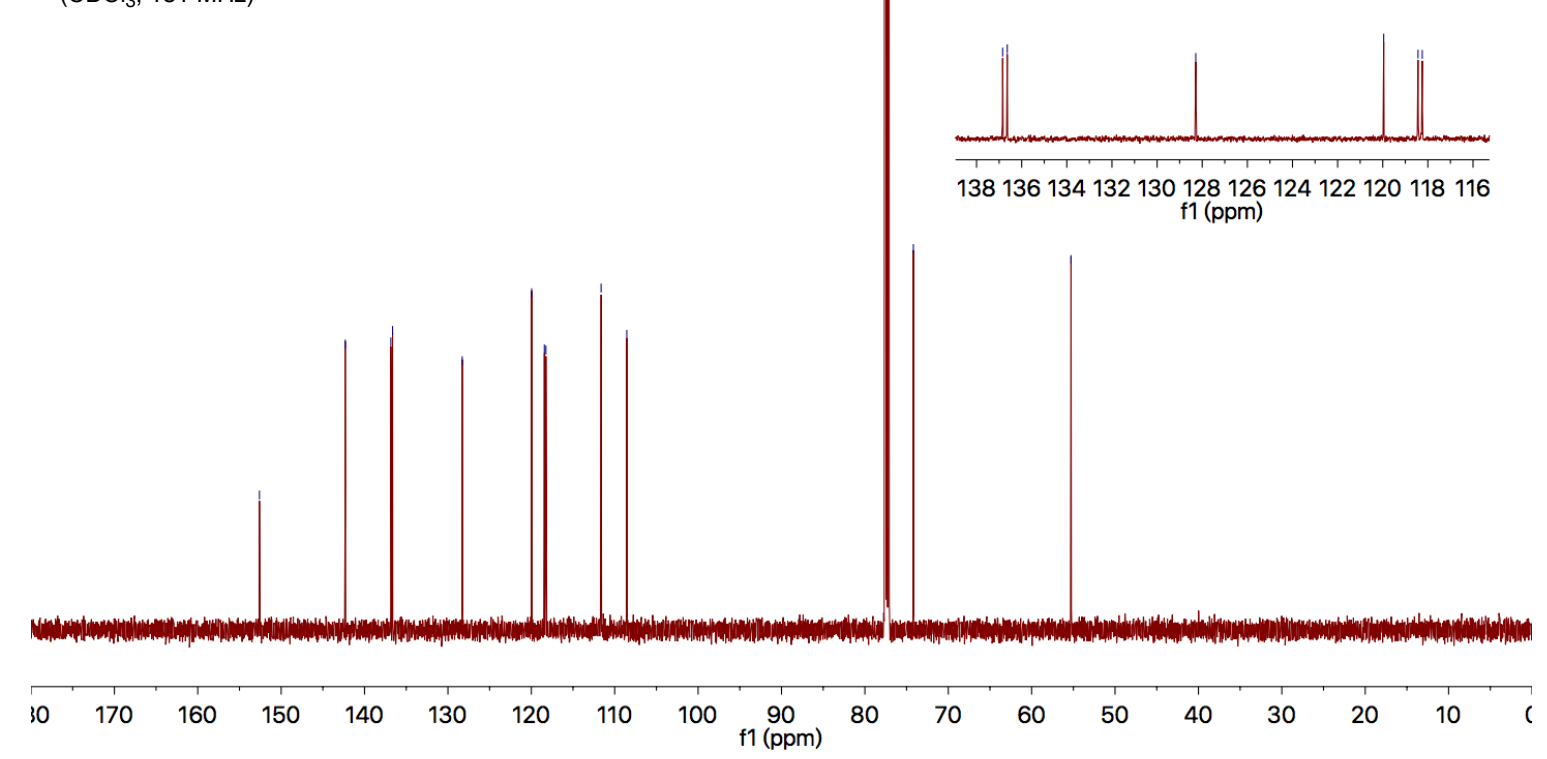



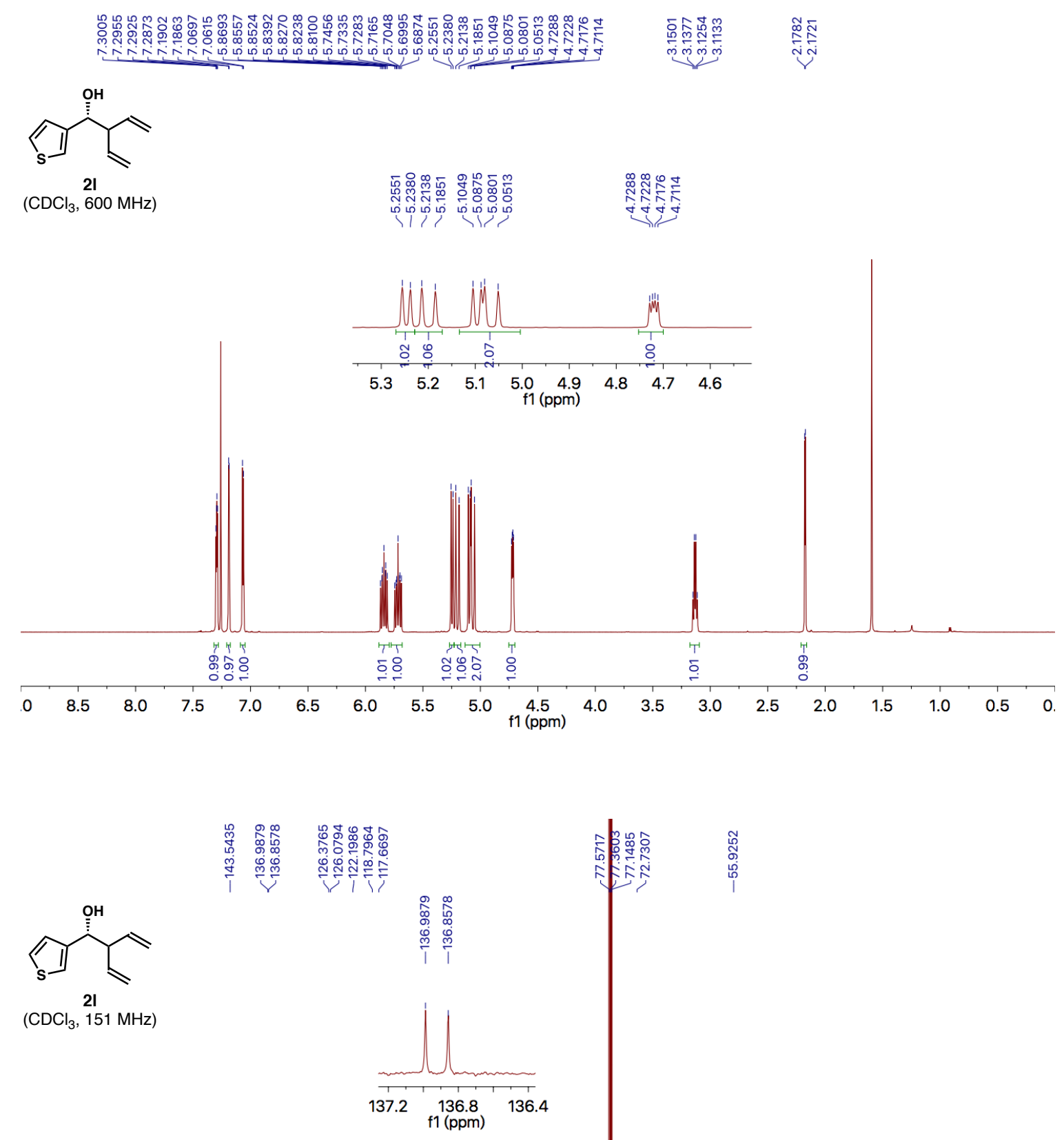

$\left(\mathrm{CDCl}_{3}, 151 \mathrm{MHz}\right)$
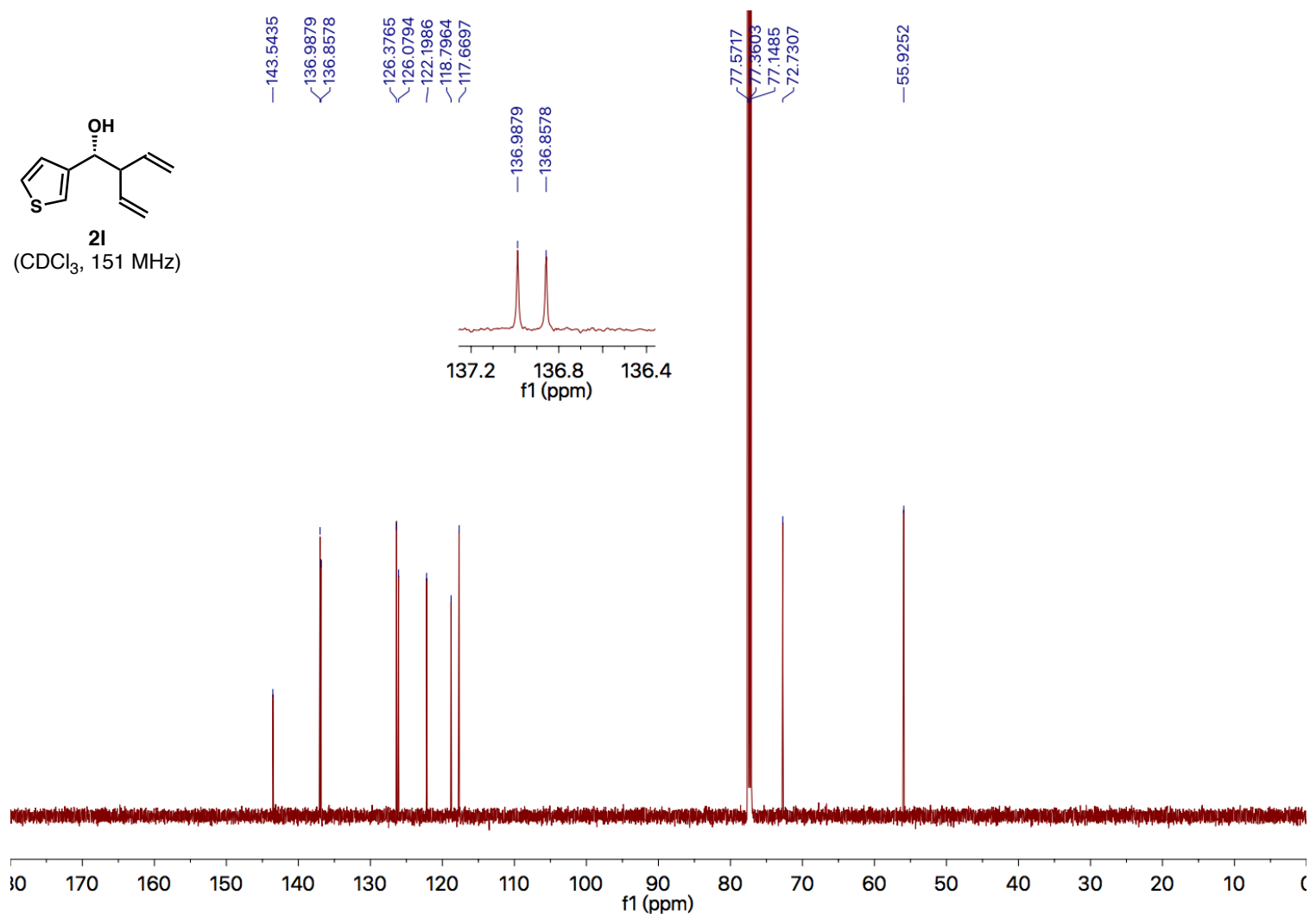

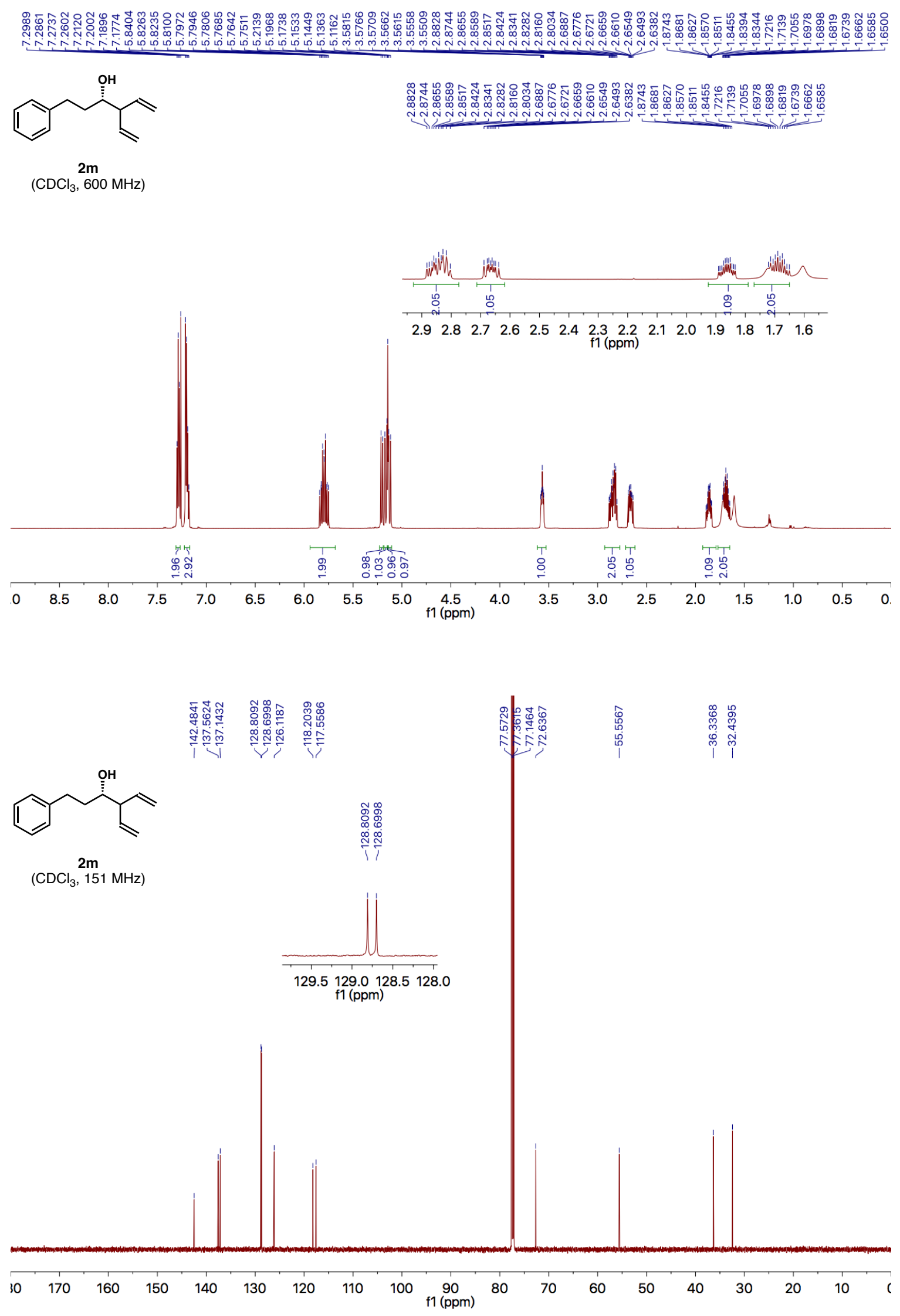

SI-48 

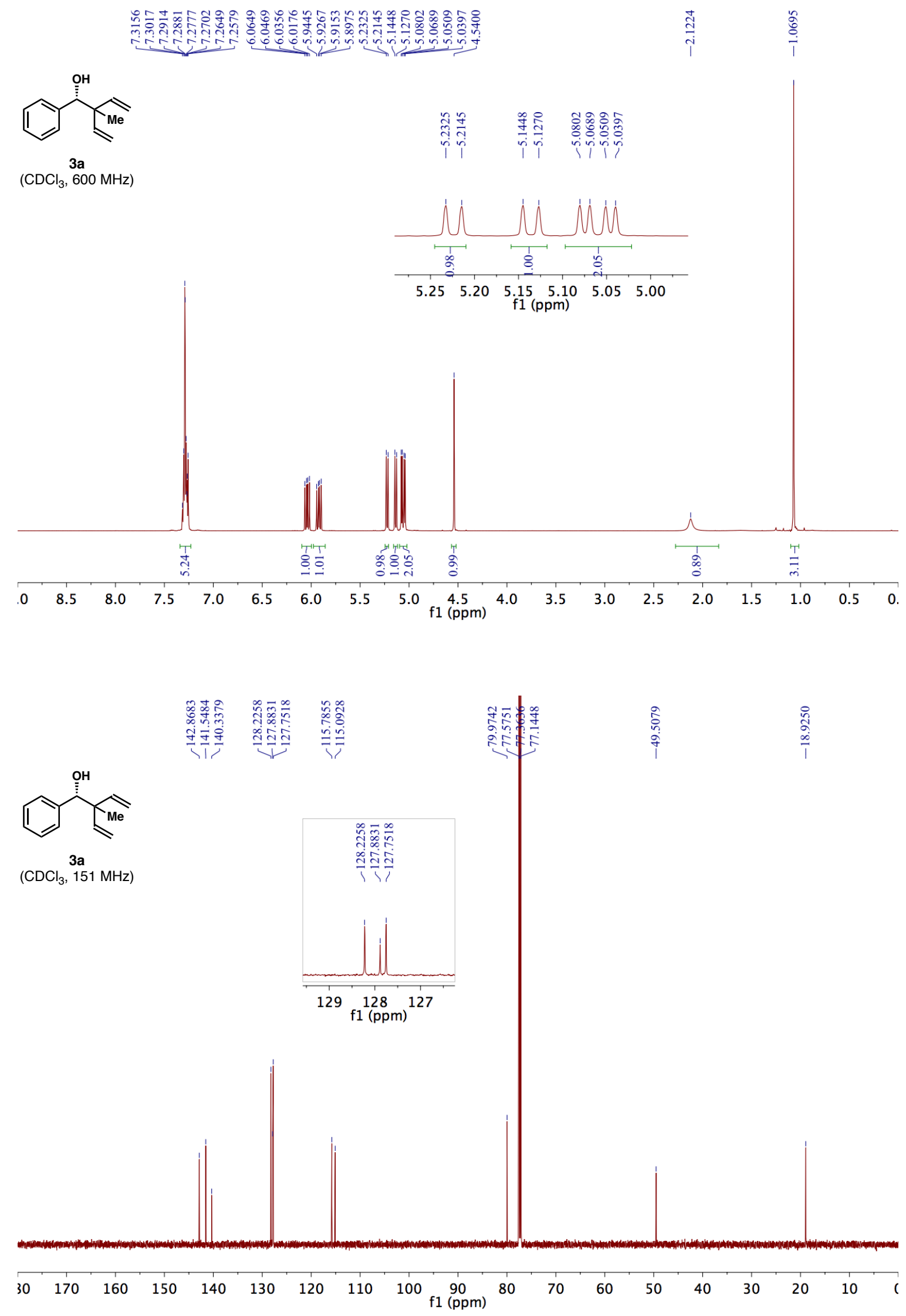

SI-49 

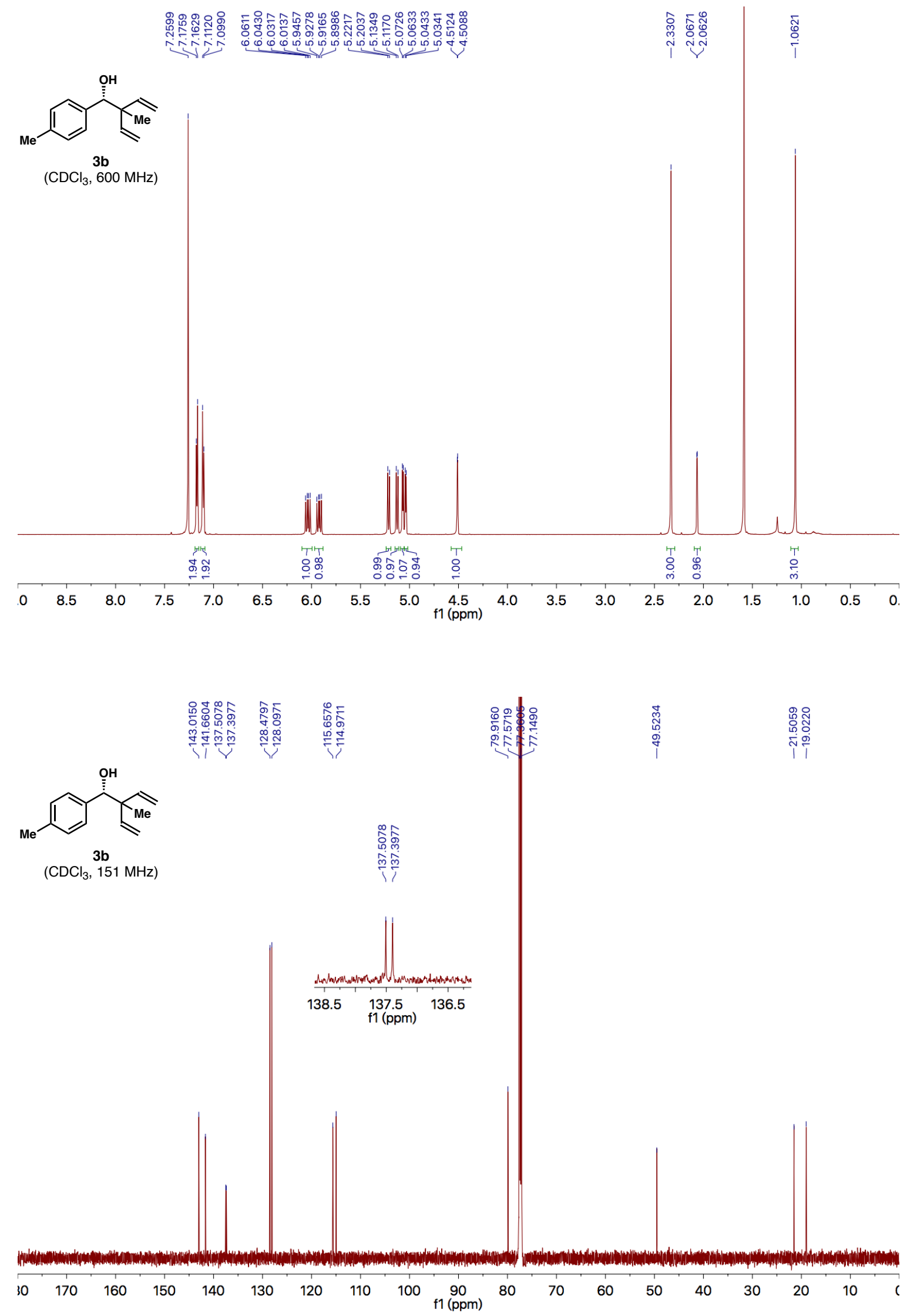

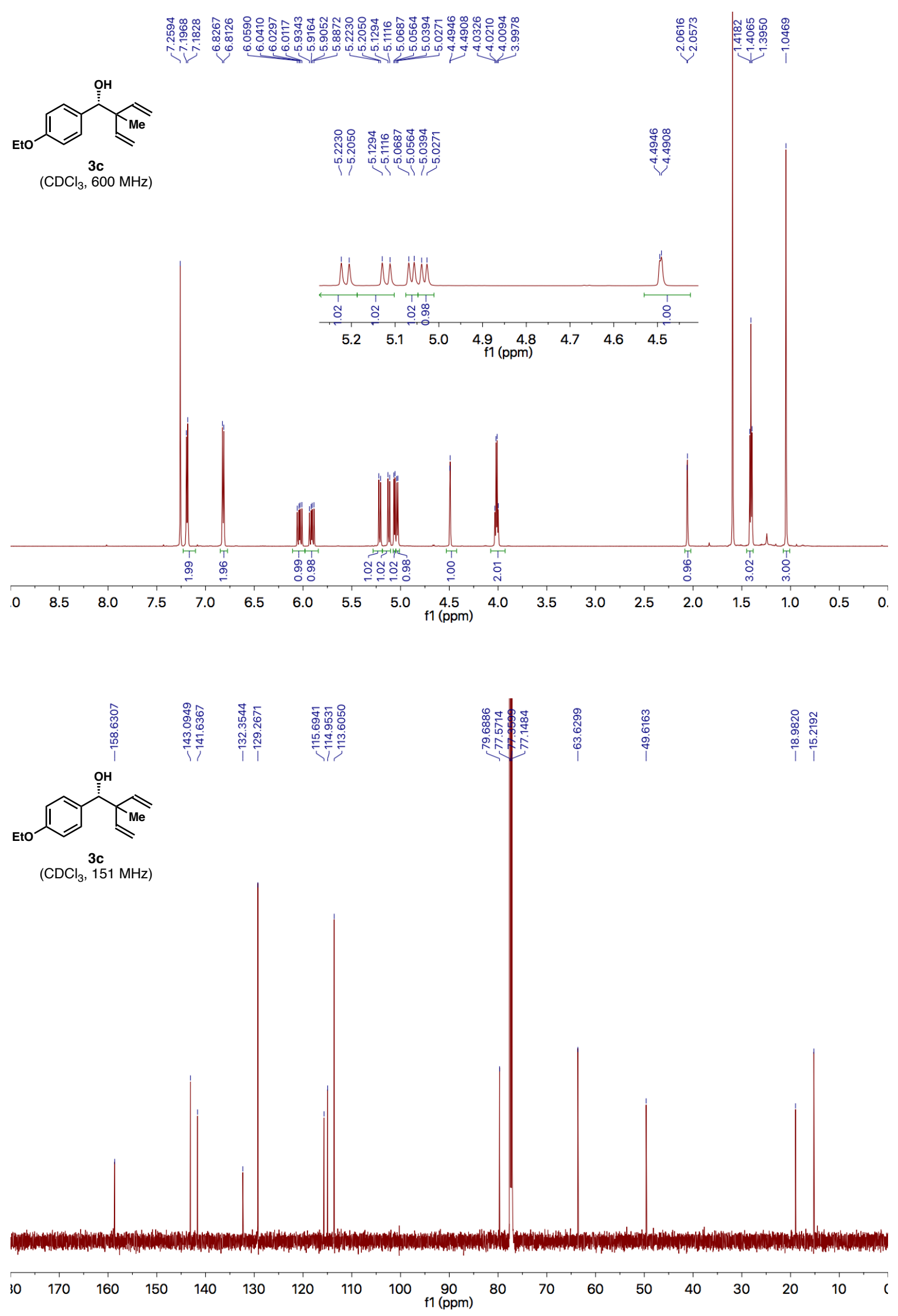

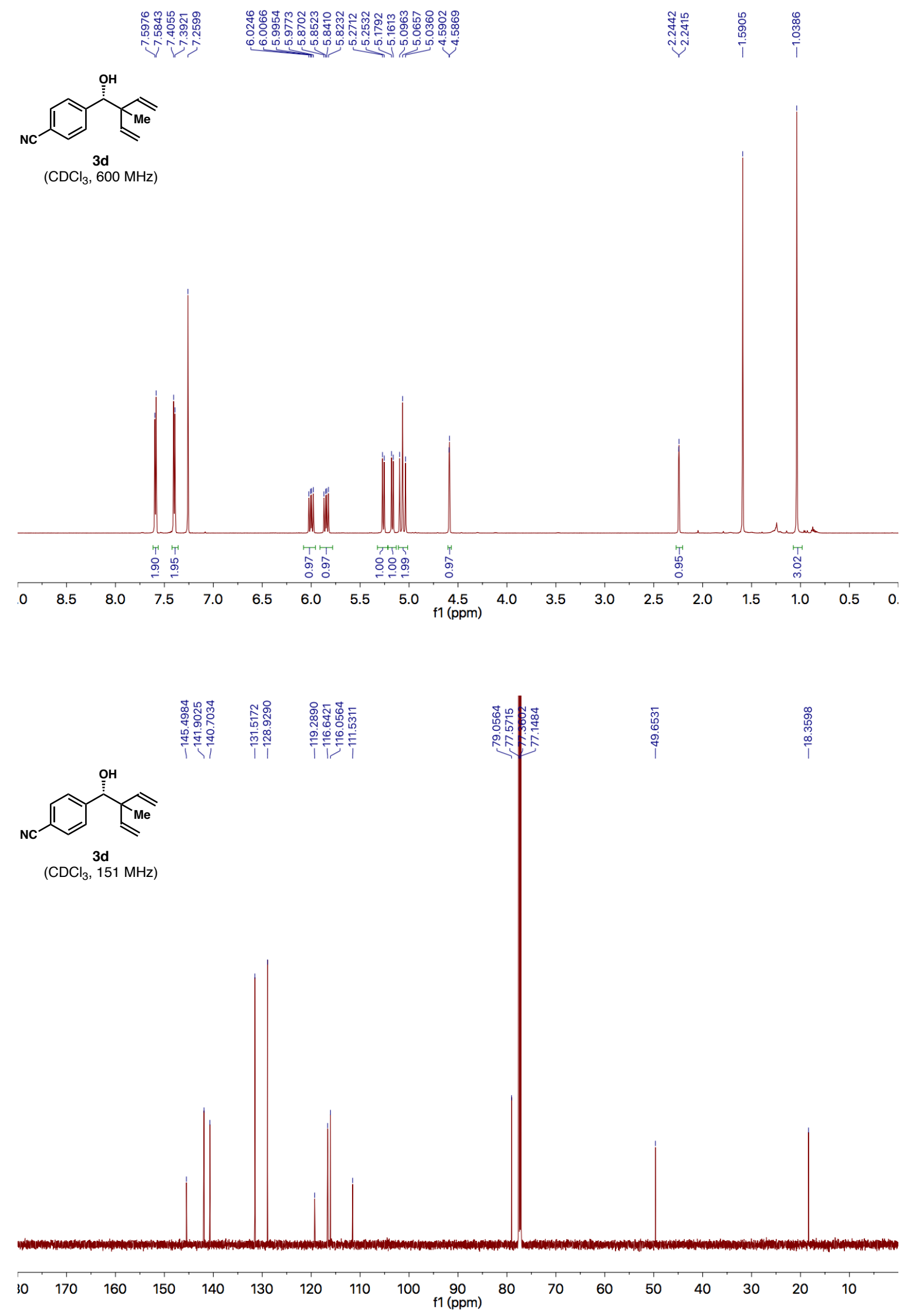

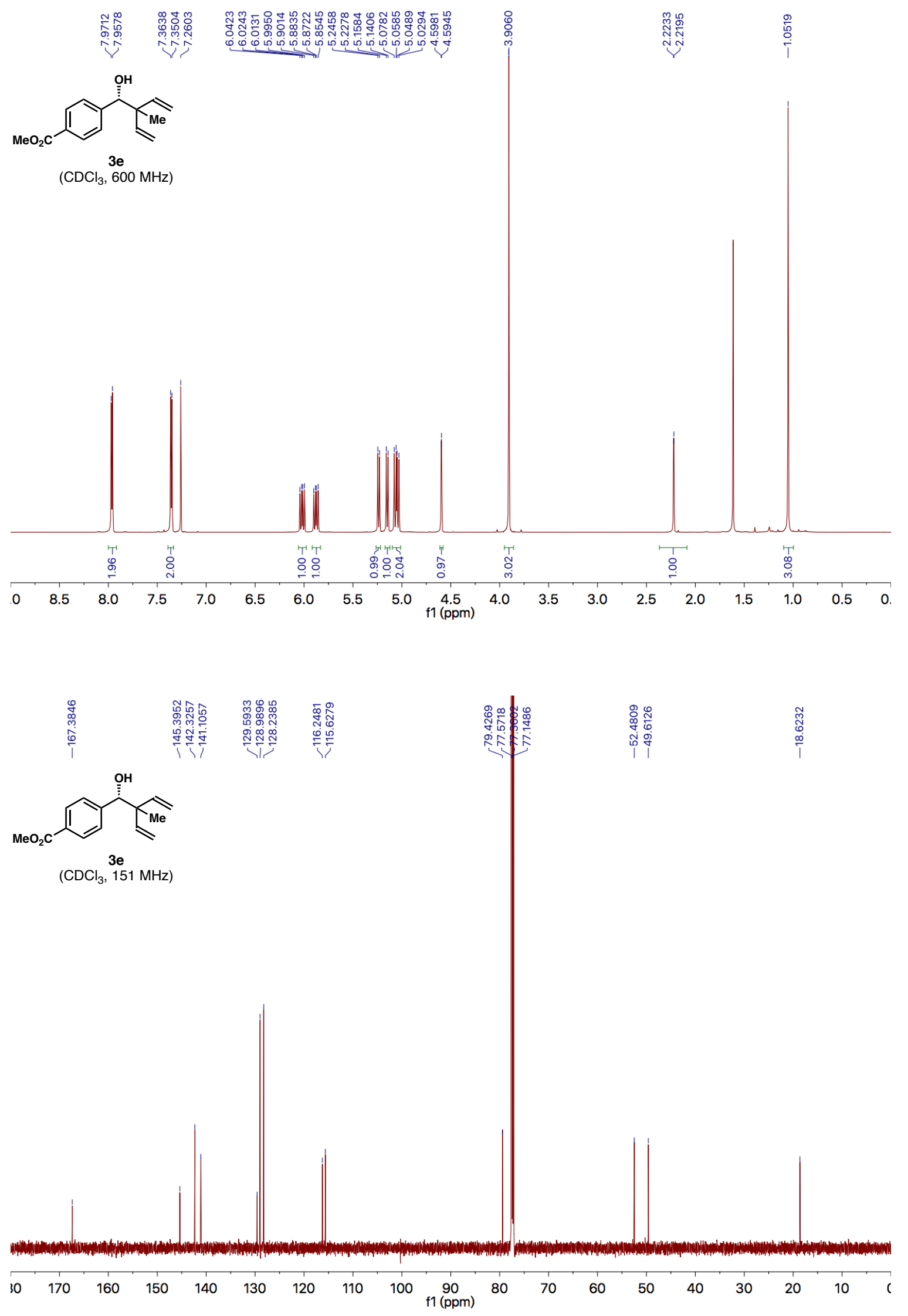

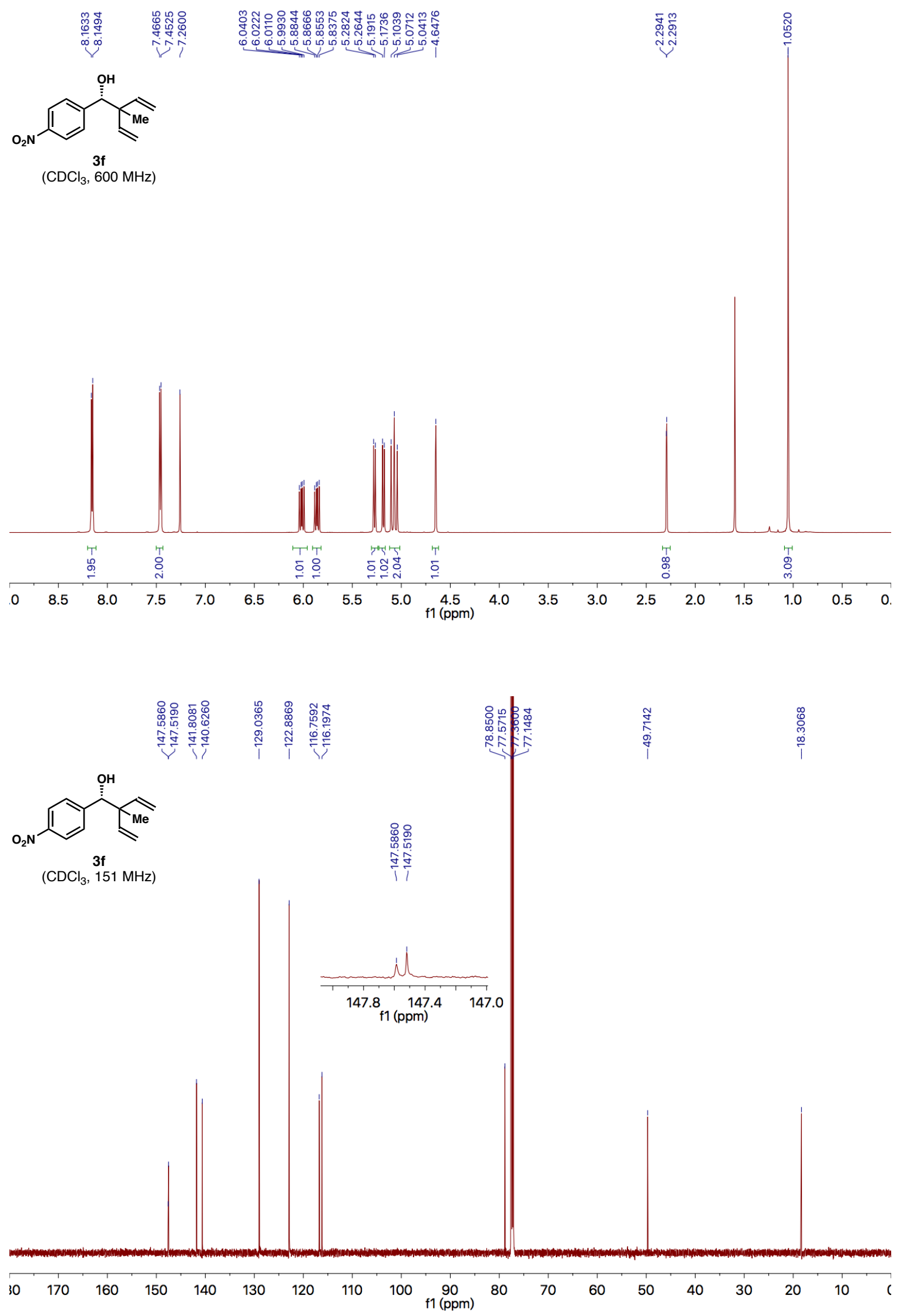

SI-54 

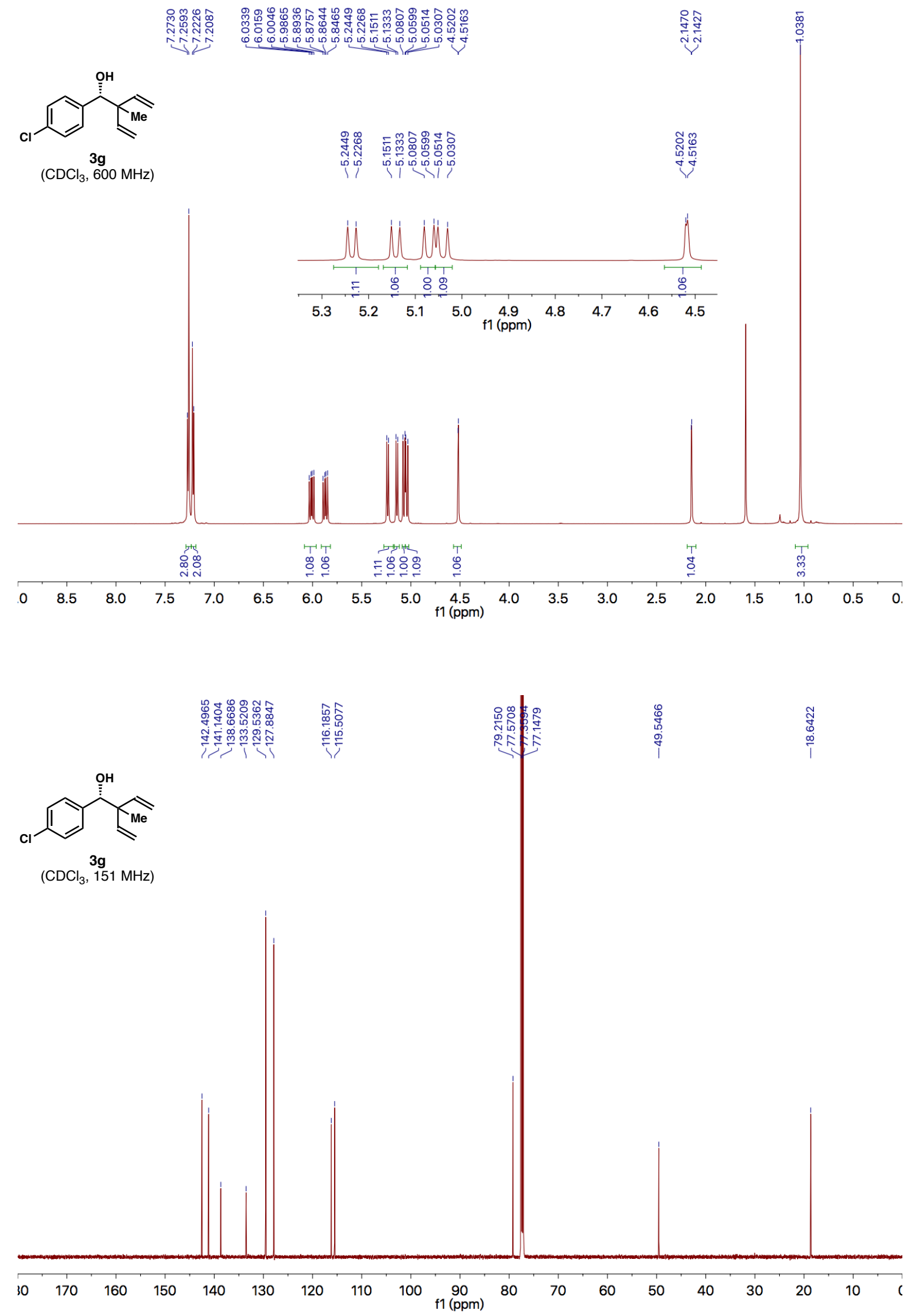

SI-55 

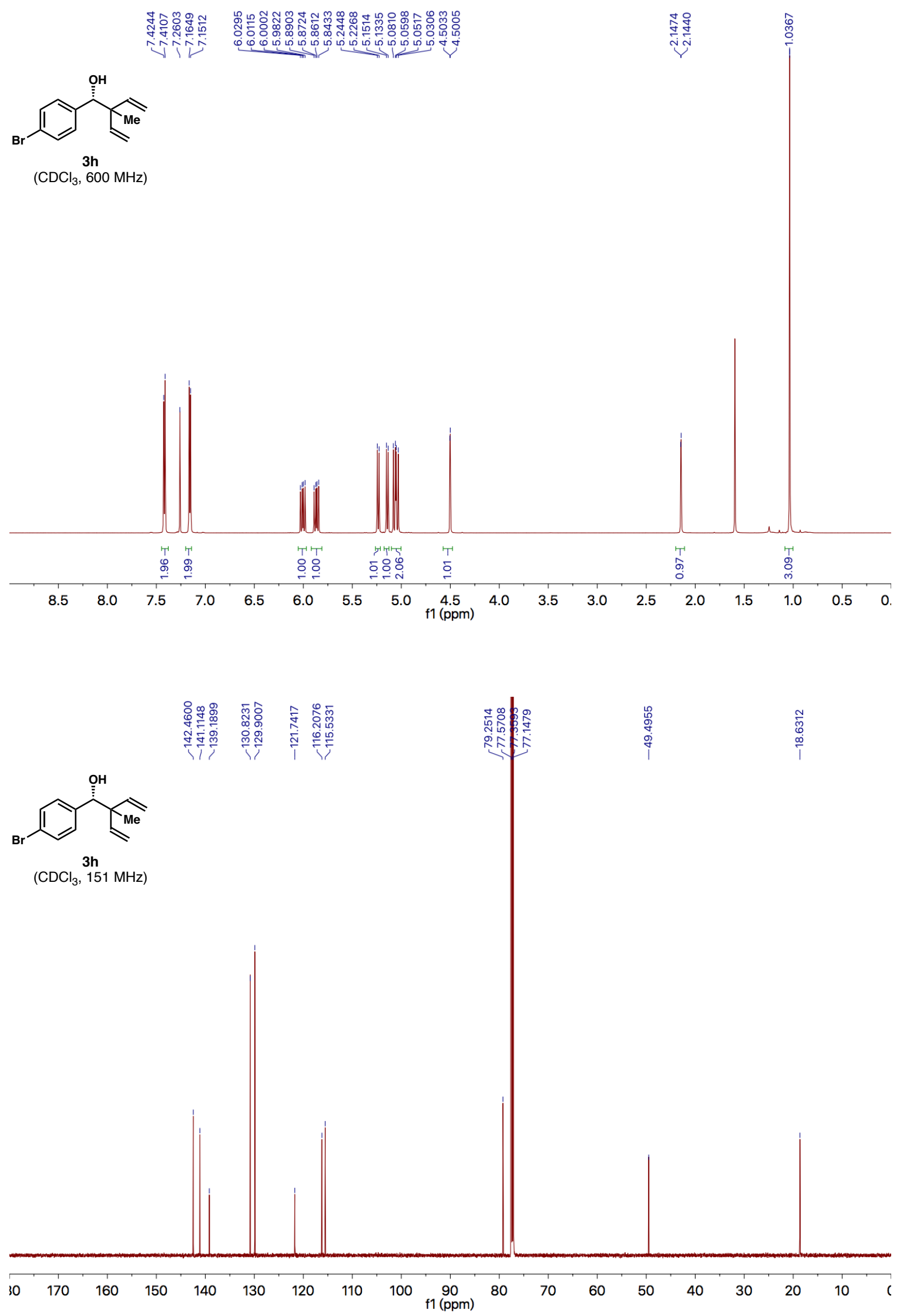

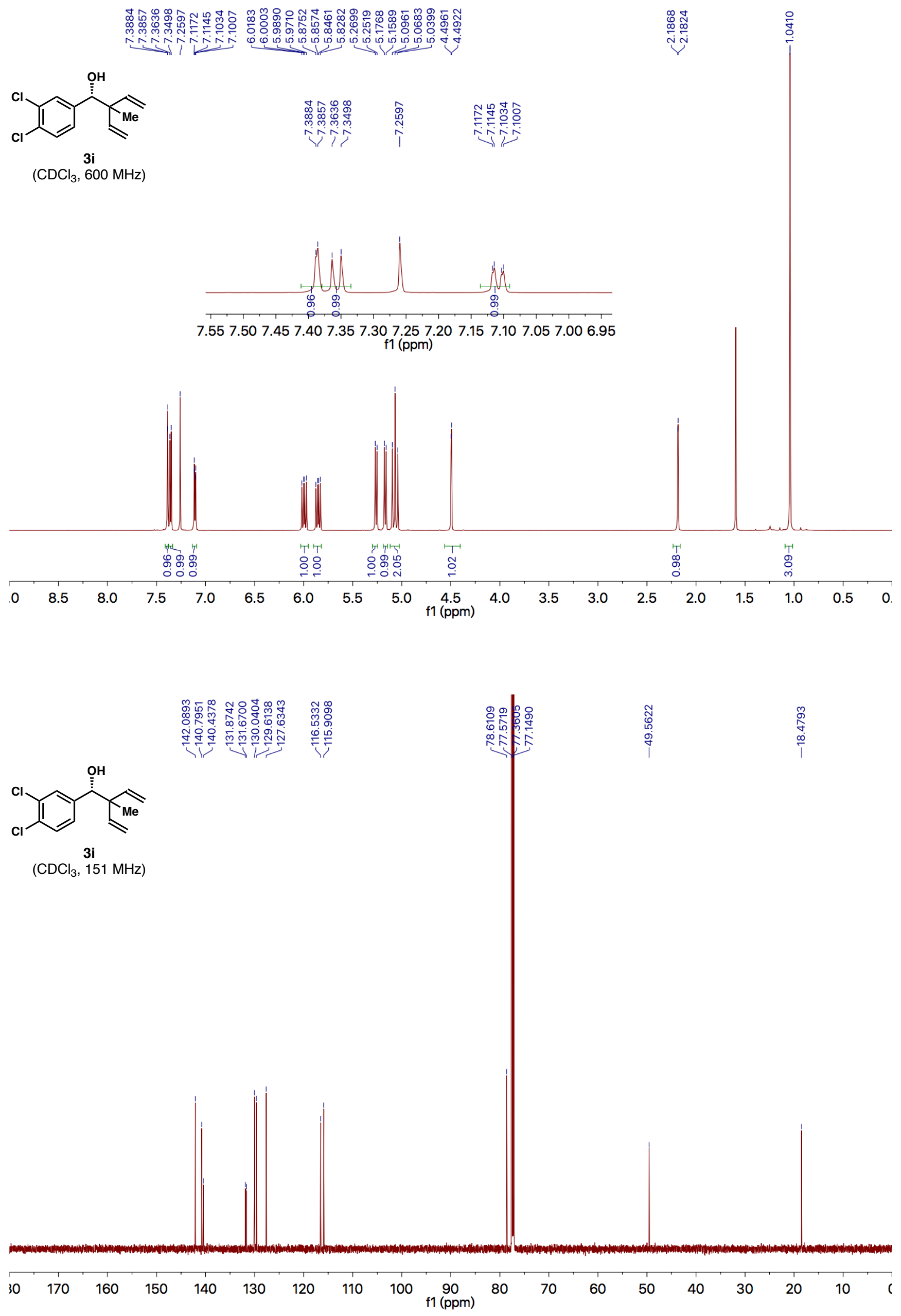

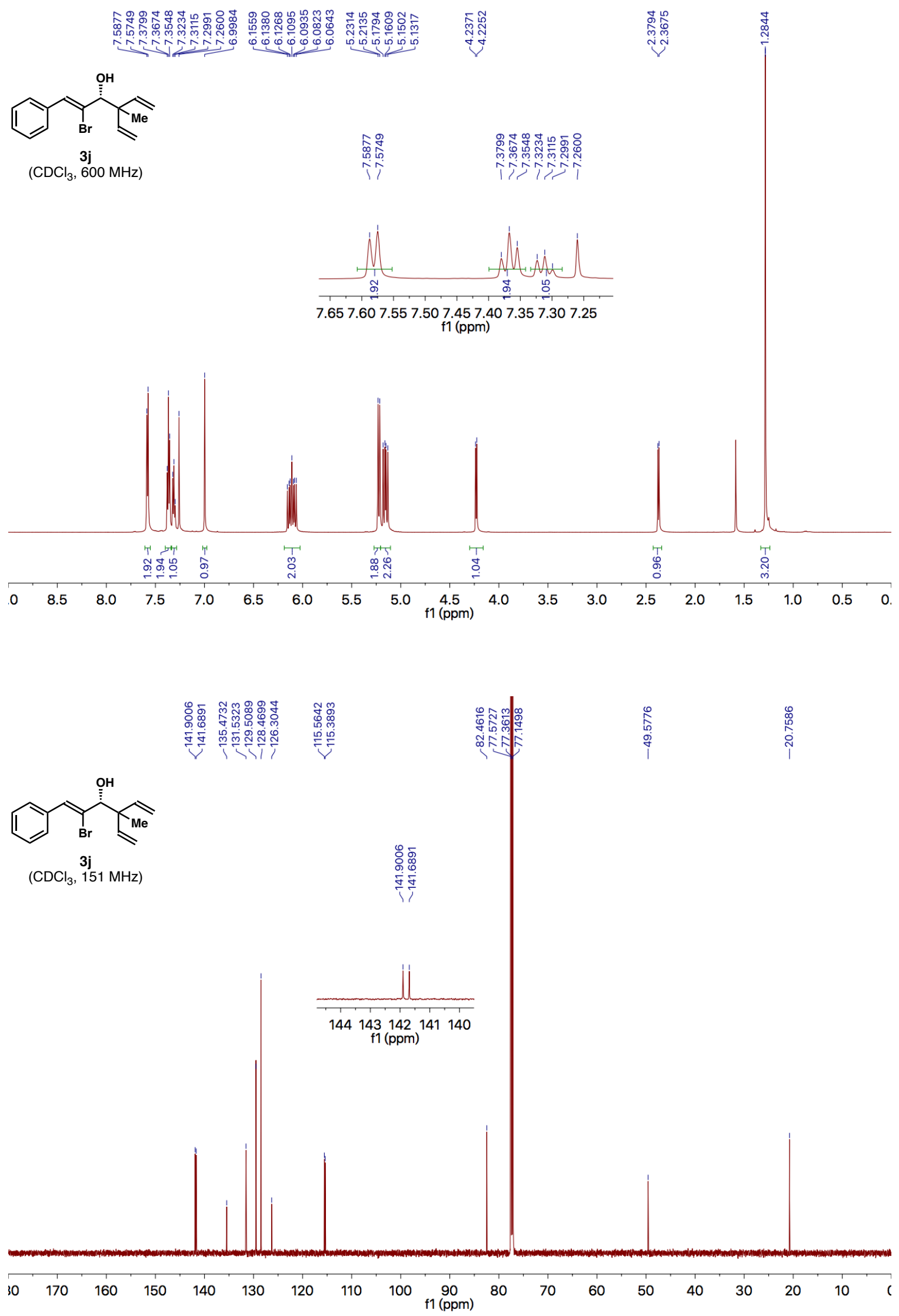

SI-58 


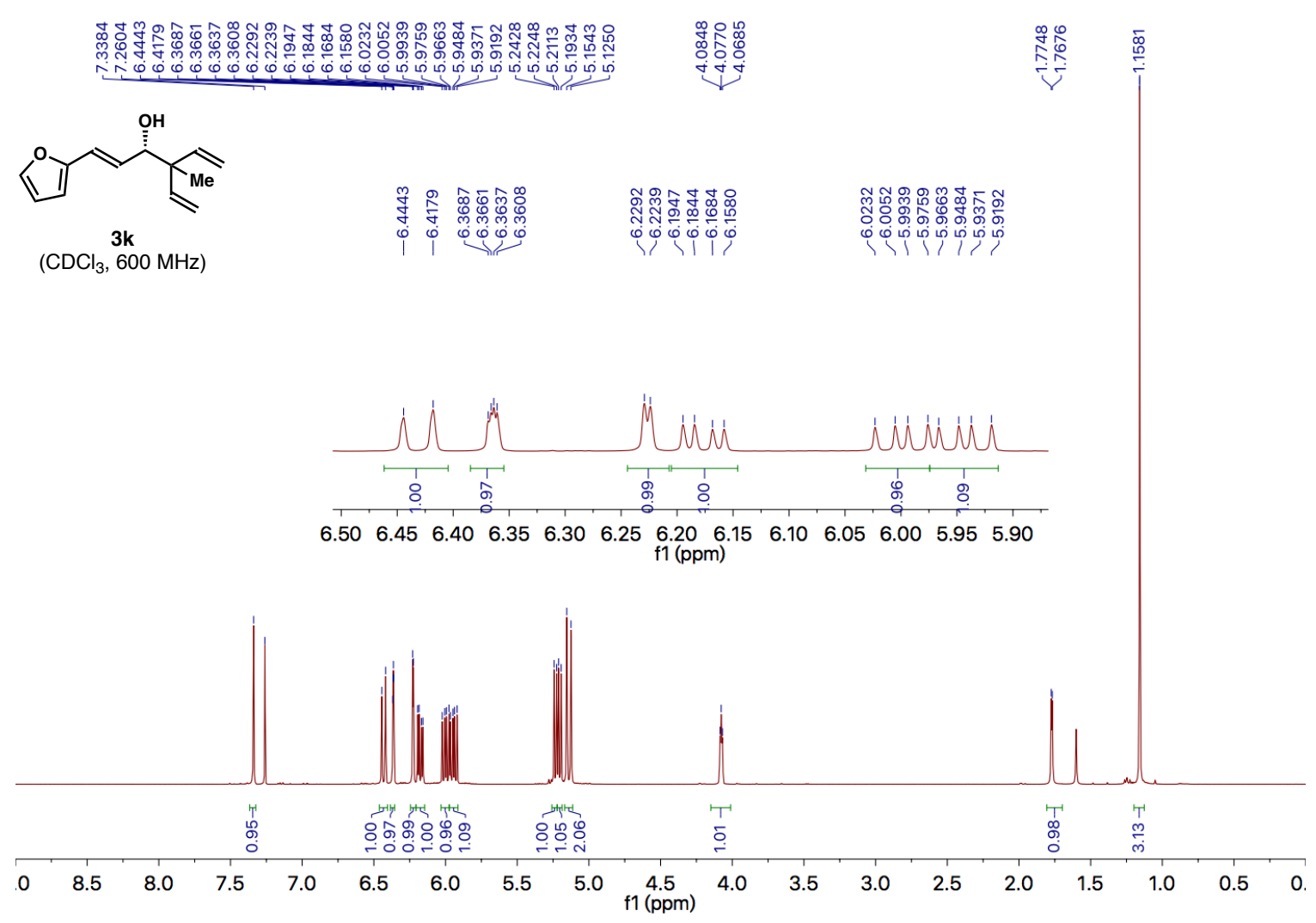

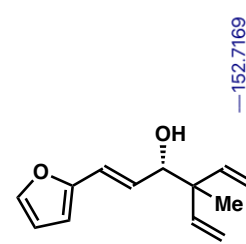

3k

$\left(\mathrm{CDCl}_{3}, 151 \mathrm{MHz}\right)$
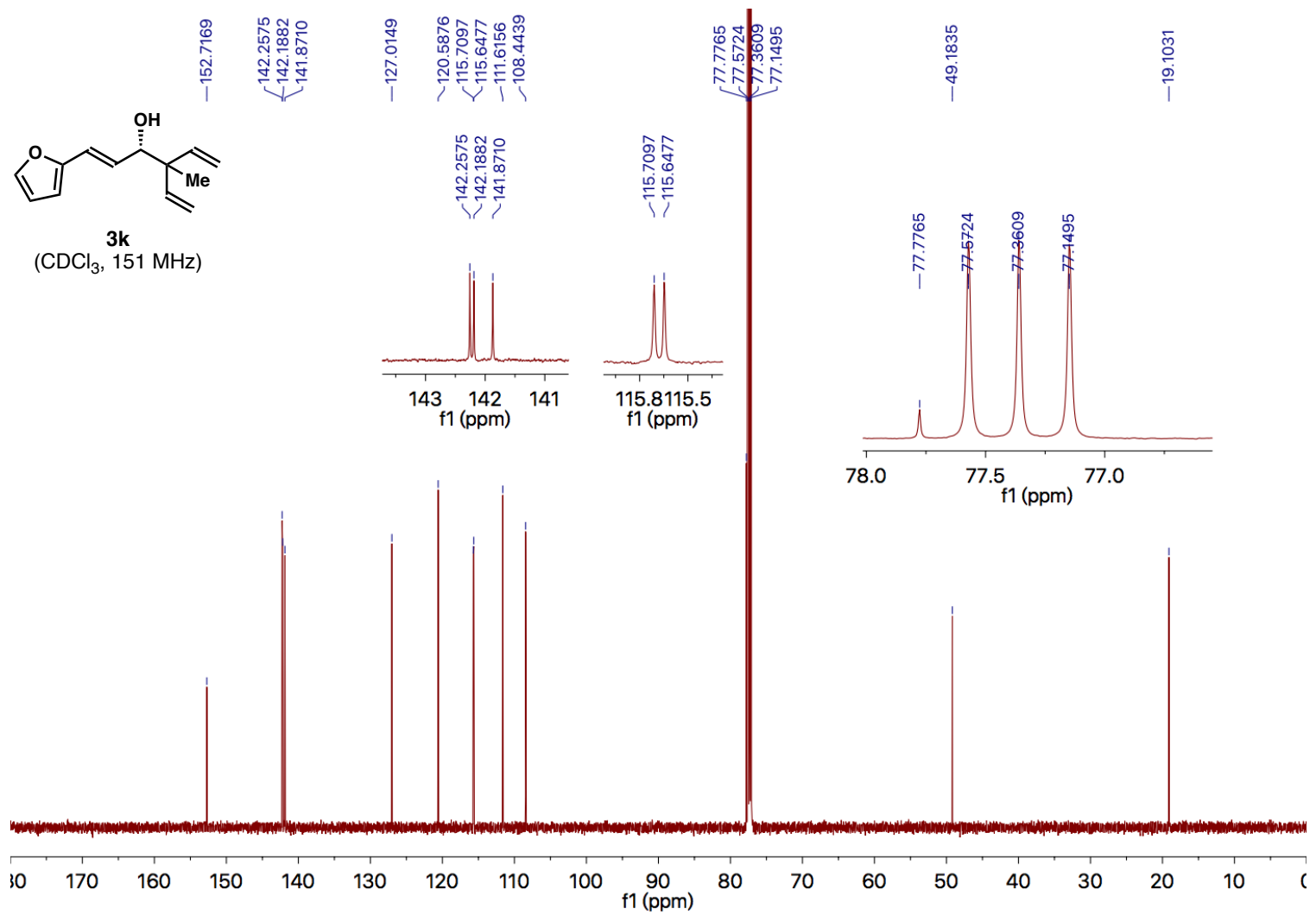

SI-59 

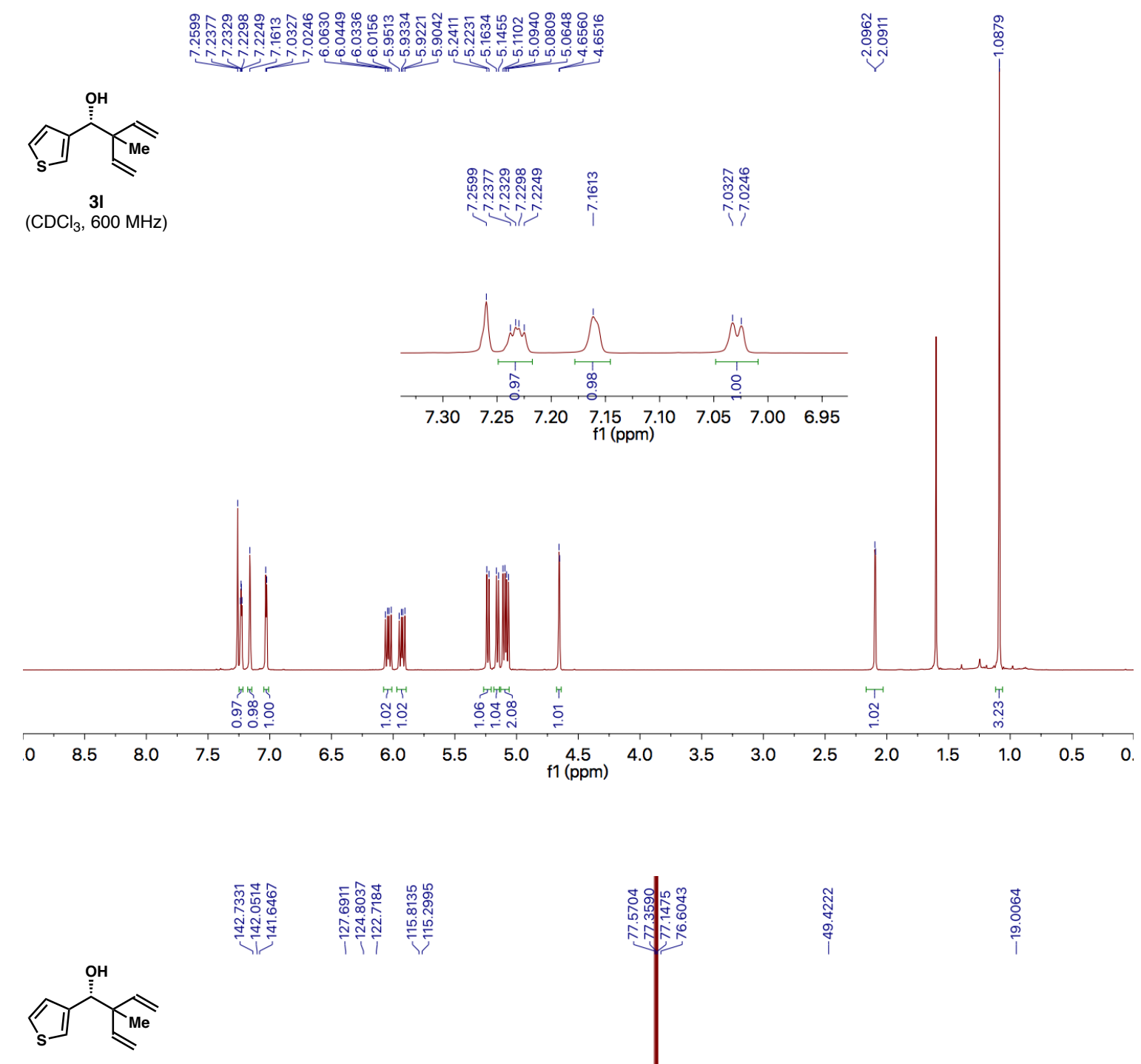

31

$\left(\mathrm{CDCl}_{3}, 151 \mathrm{MHz}\right)$

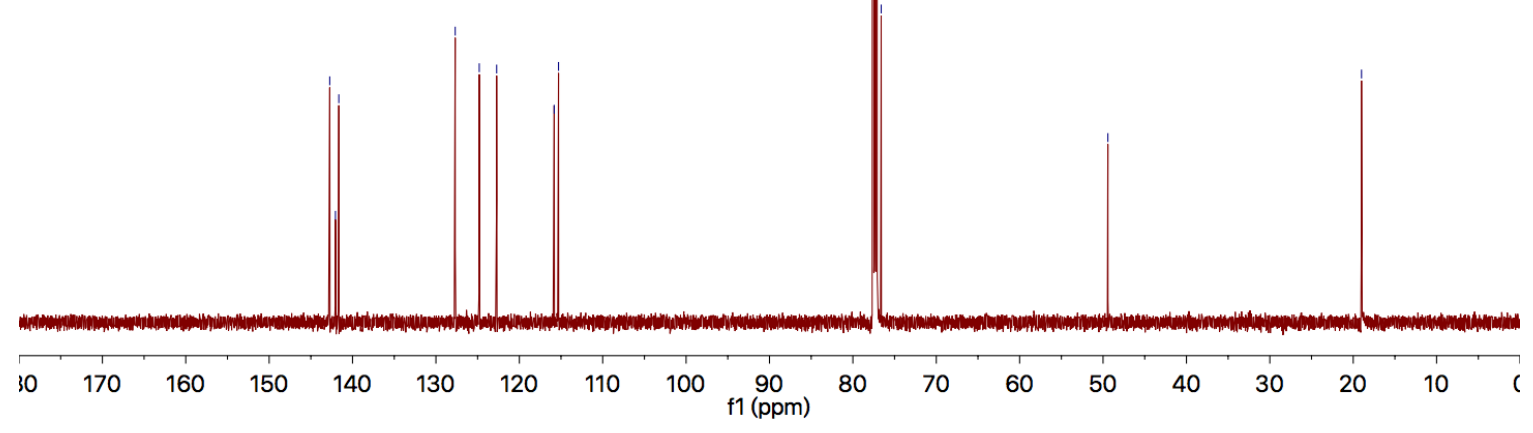



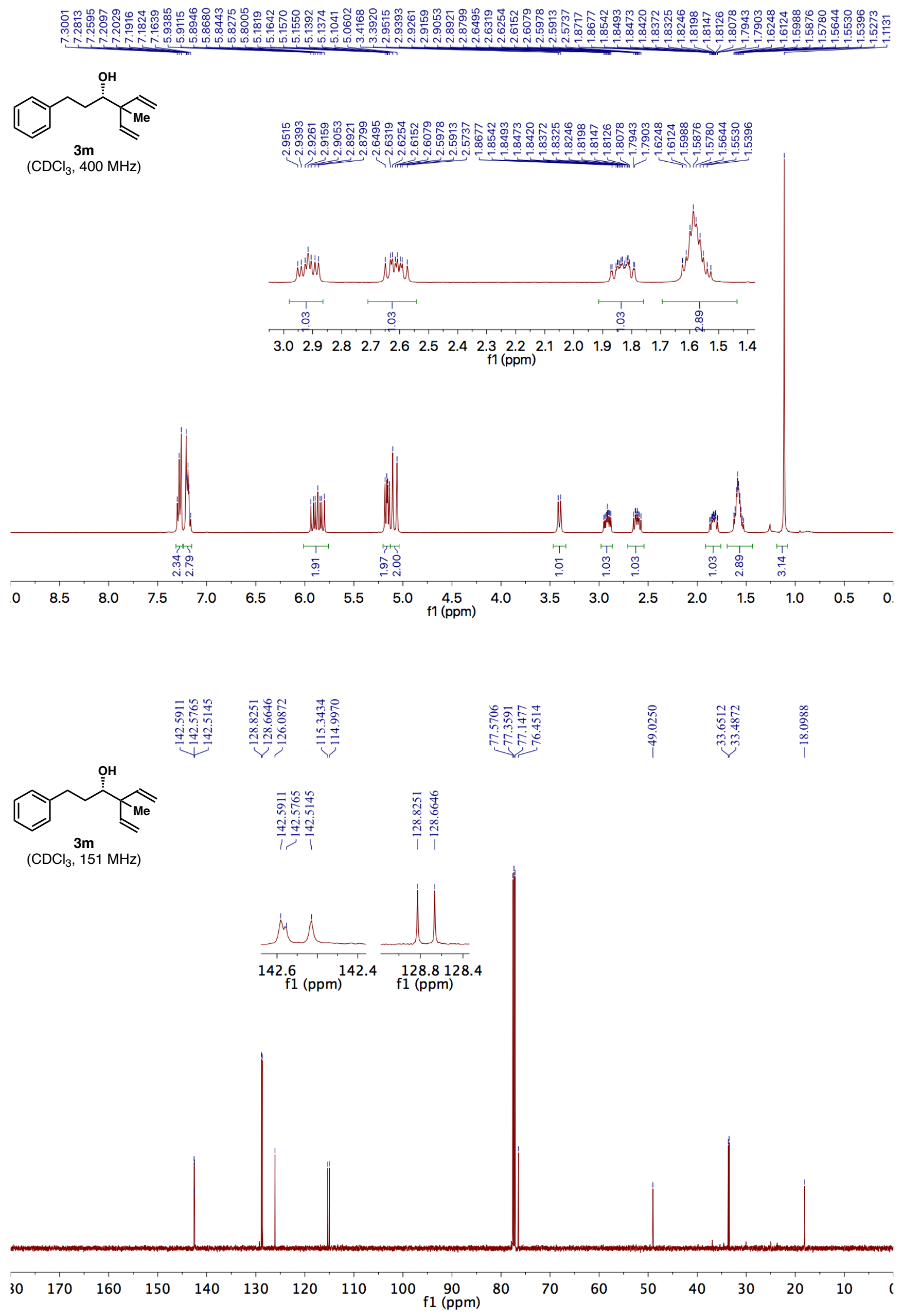

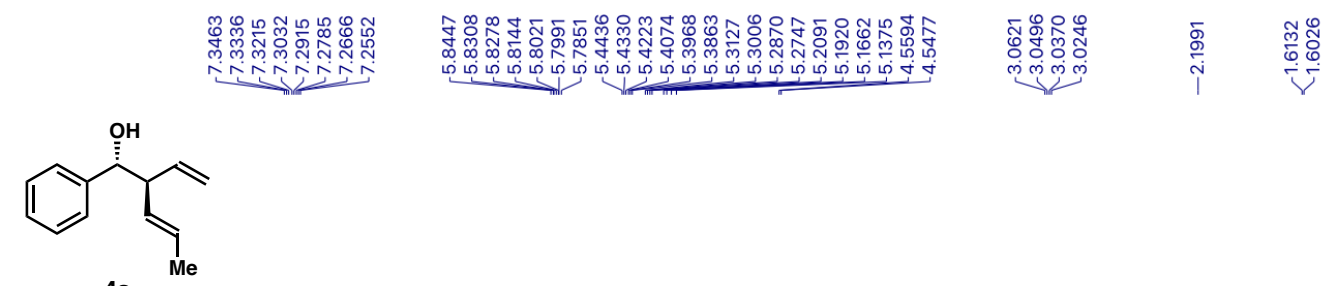

$\left(\mathrm{CDCl}_{3}, 600 \mathrm{MHz}\right)$
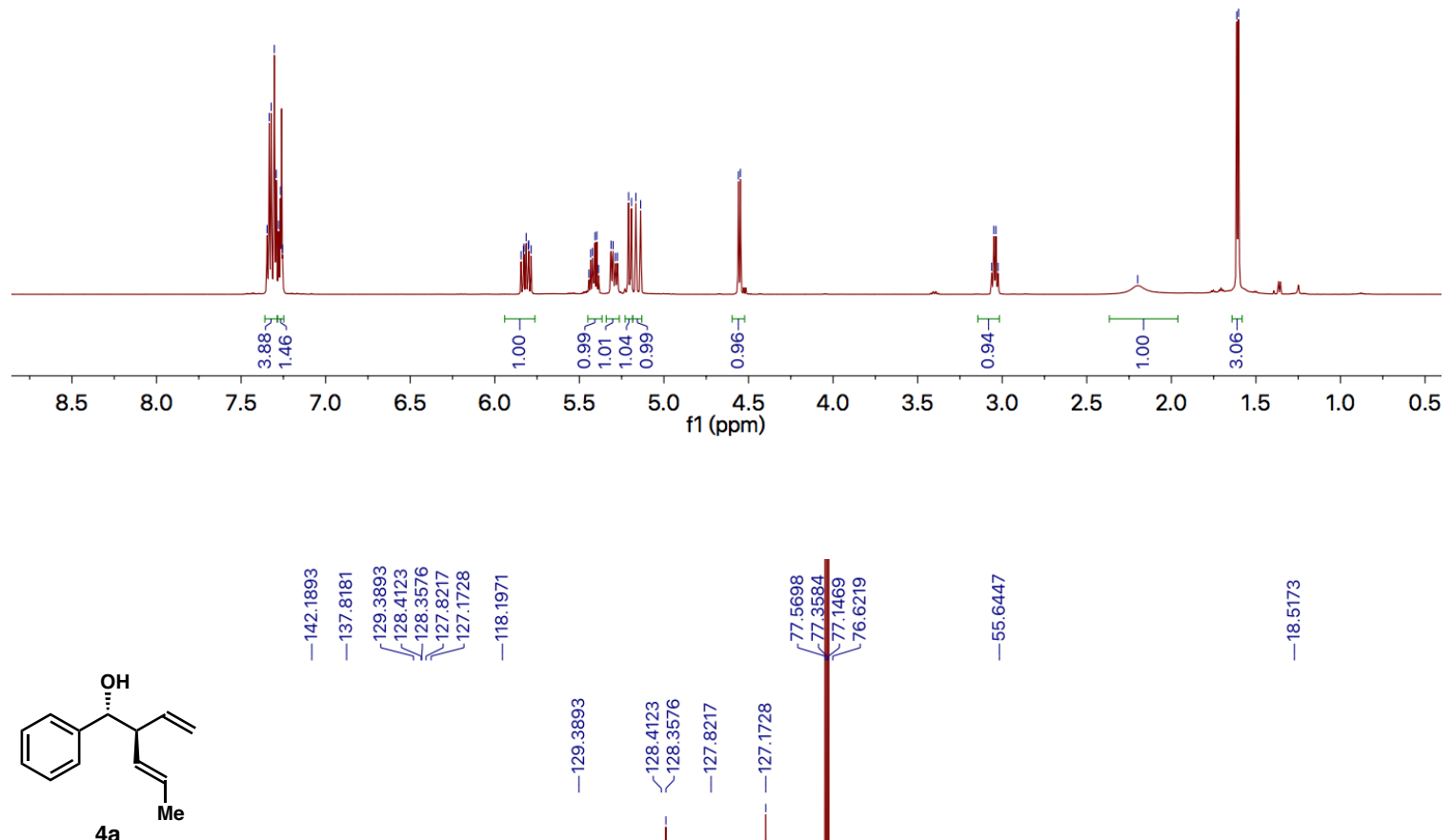

$\left(\mathrm{CDCl}_{3}, 151 \mathrm{MHz}\right)$
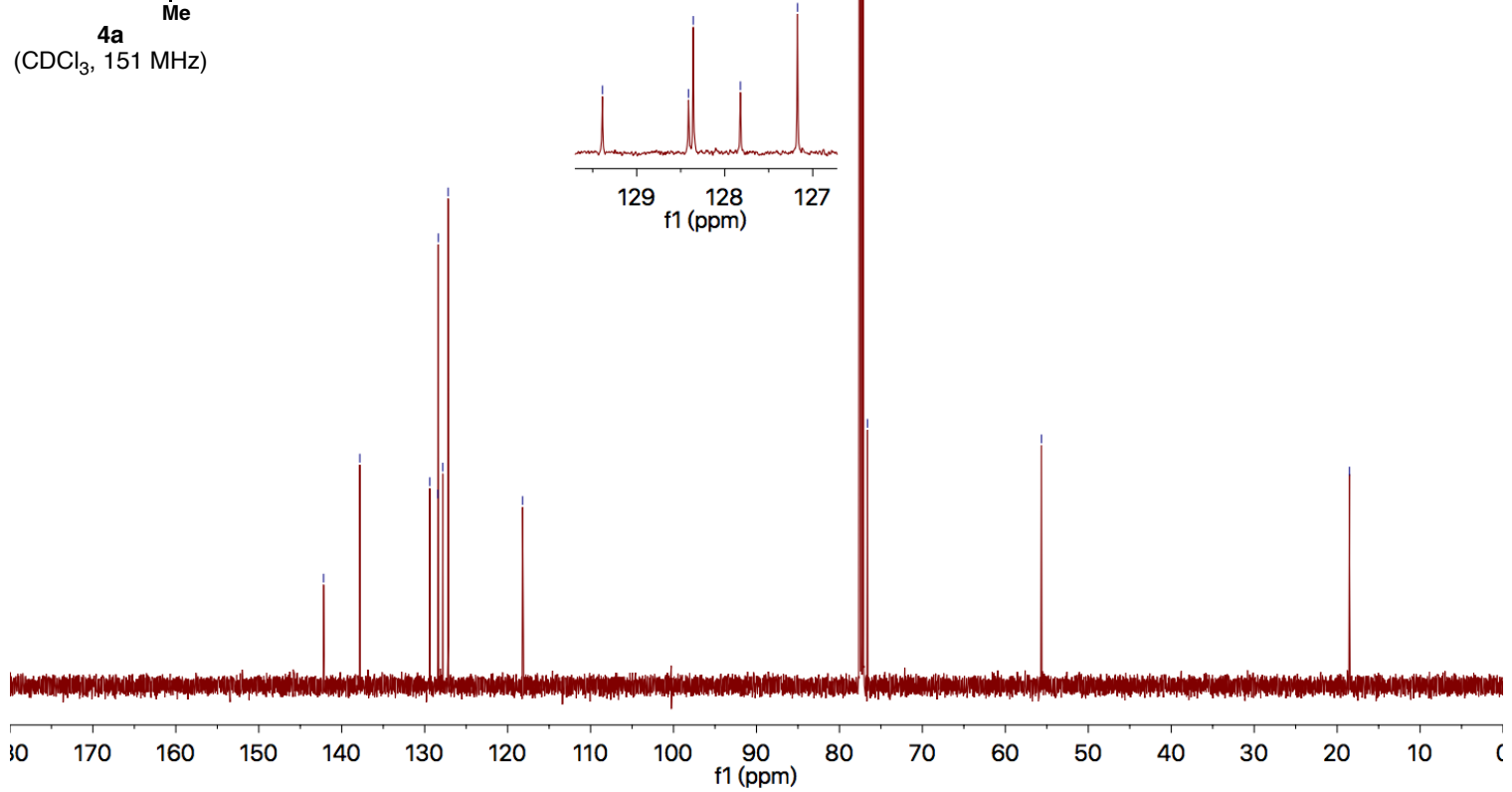

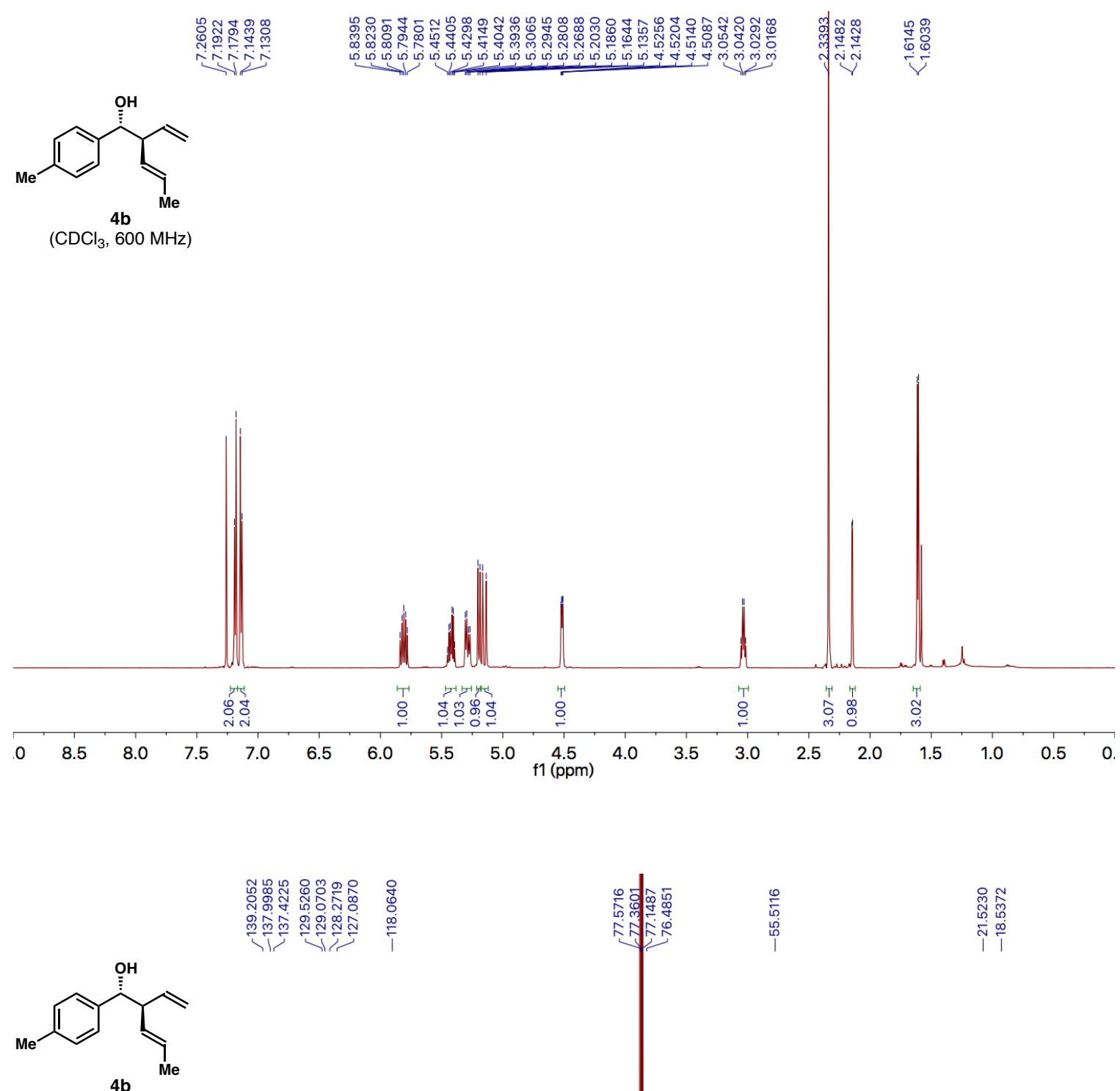

$\left(\mathrm{CDCl}_{3}, 151 \mathrm{MHz}\right)$

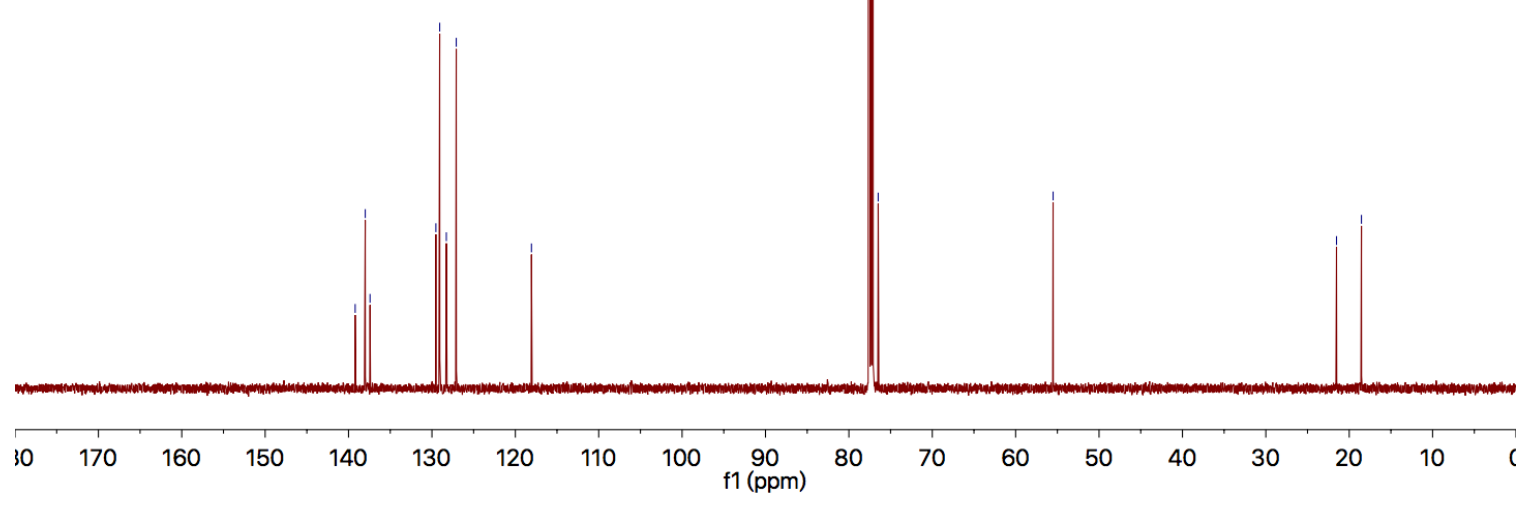



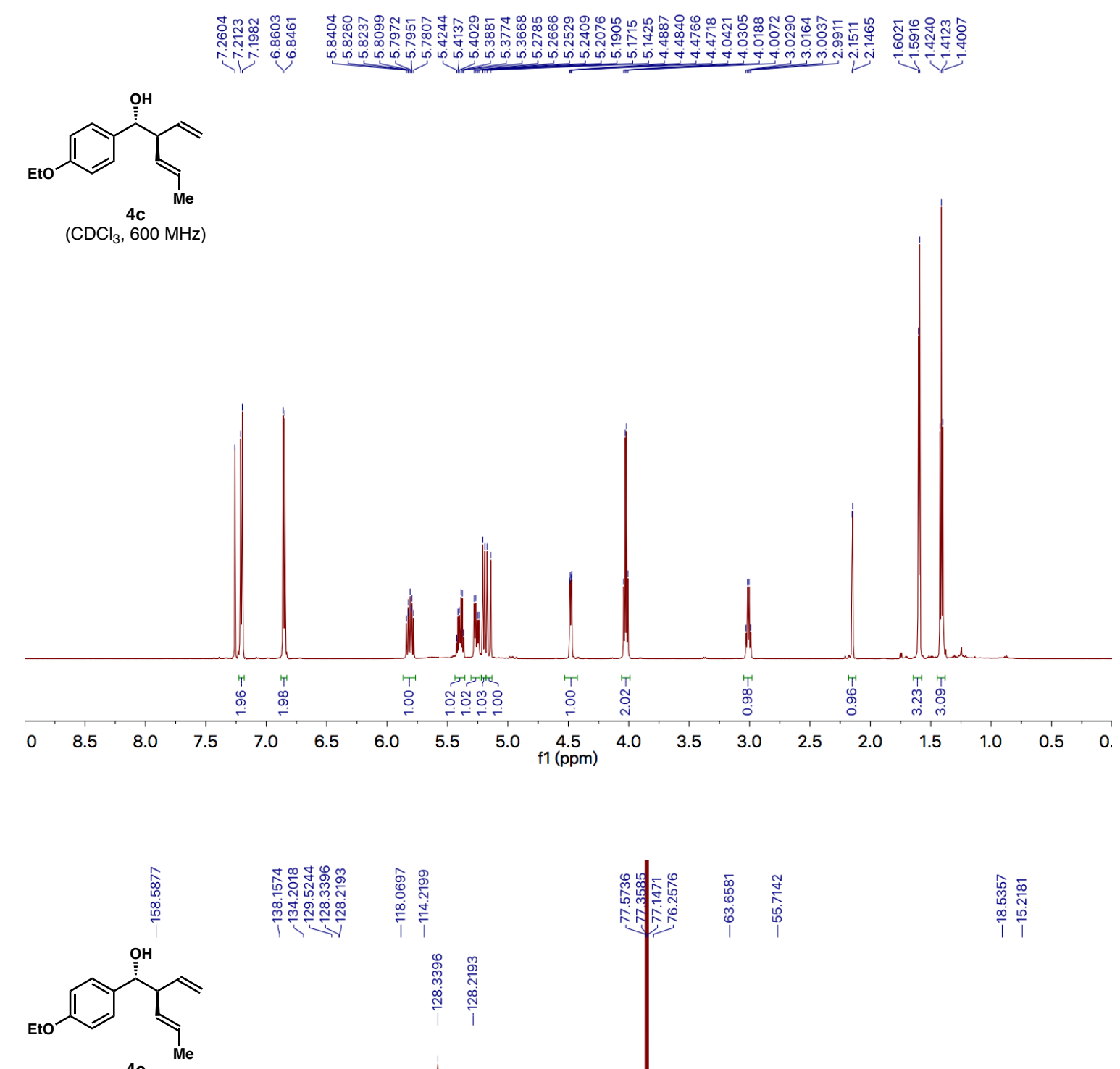

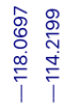

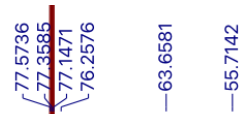

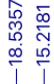

$\left(\mathrm{CDCl}_{3}, 151 \mathrm{MHz}\right)$
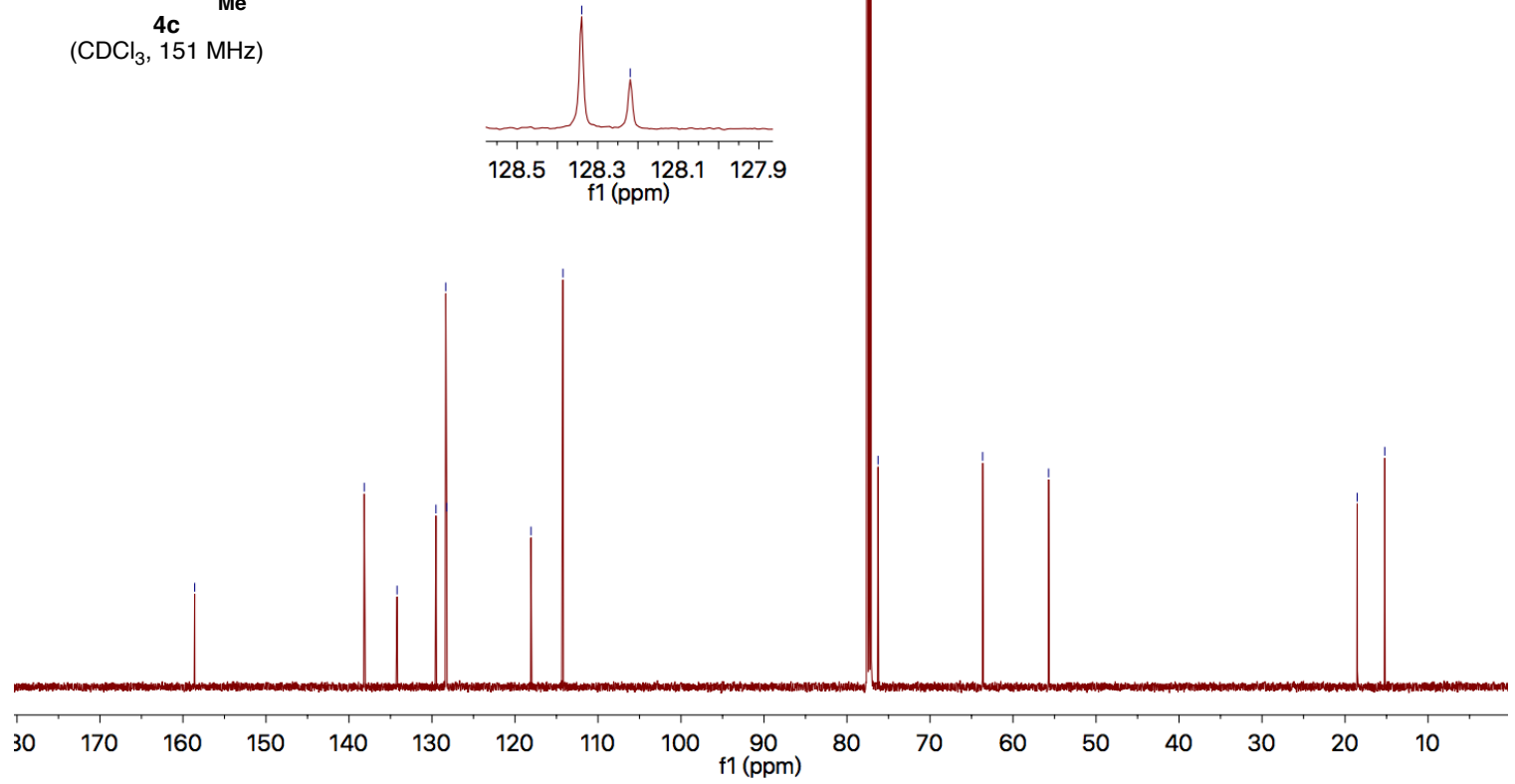

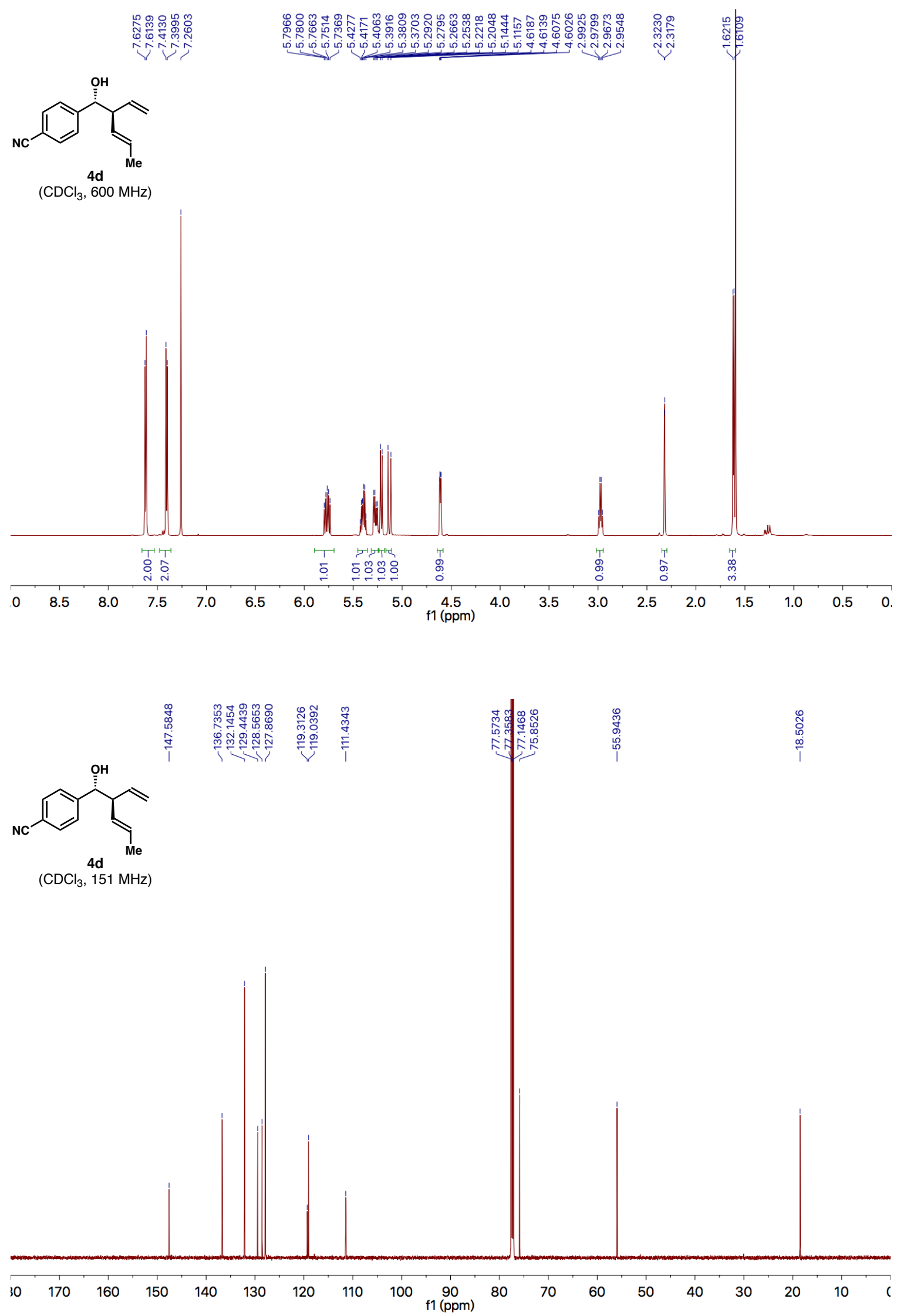

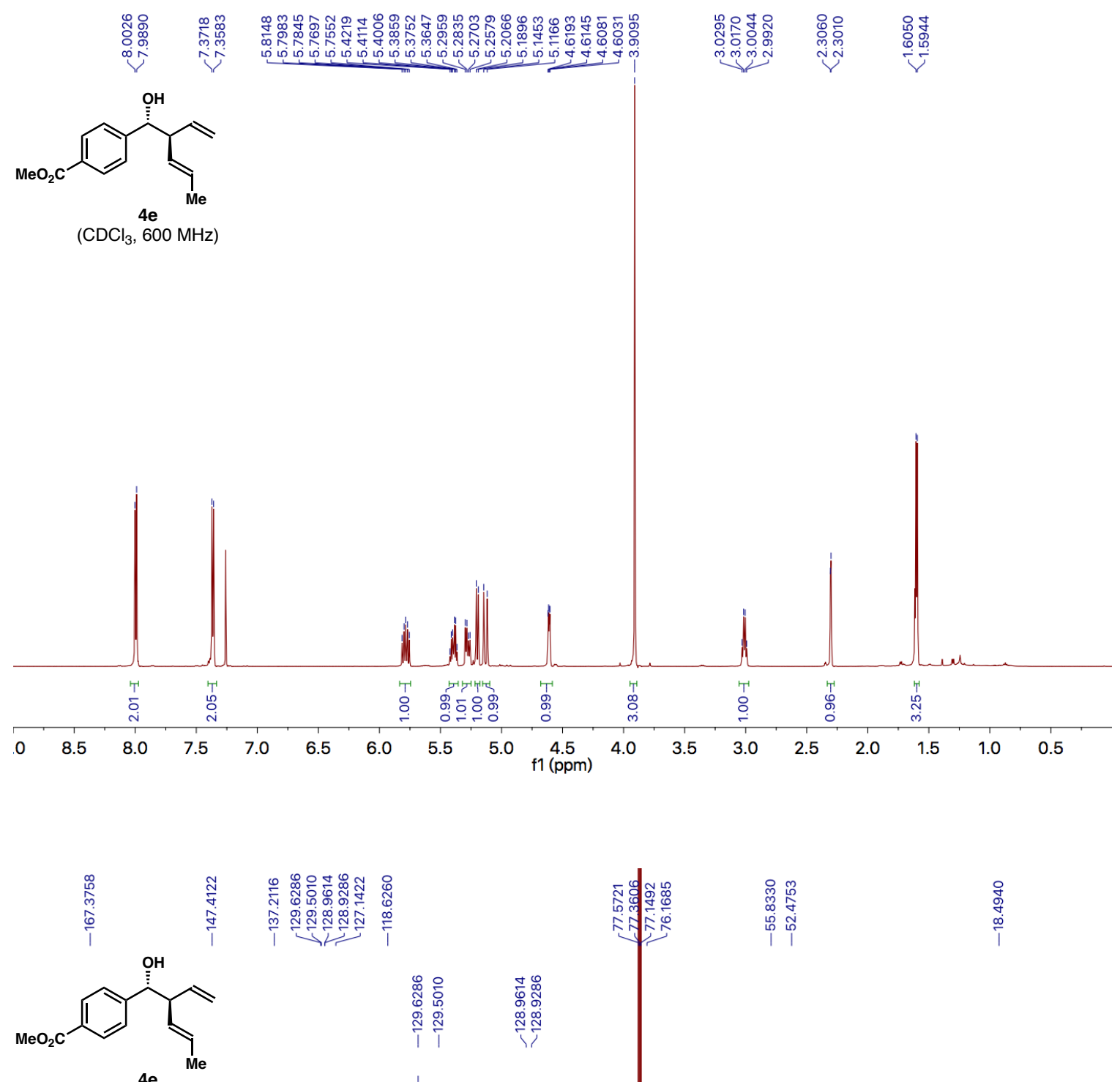

$\left(\mathrm{CDCl}_{3}, 151 \mathrm{MHz}\right)$
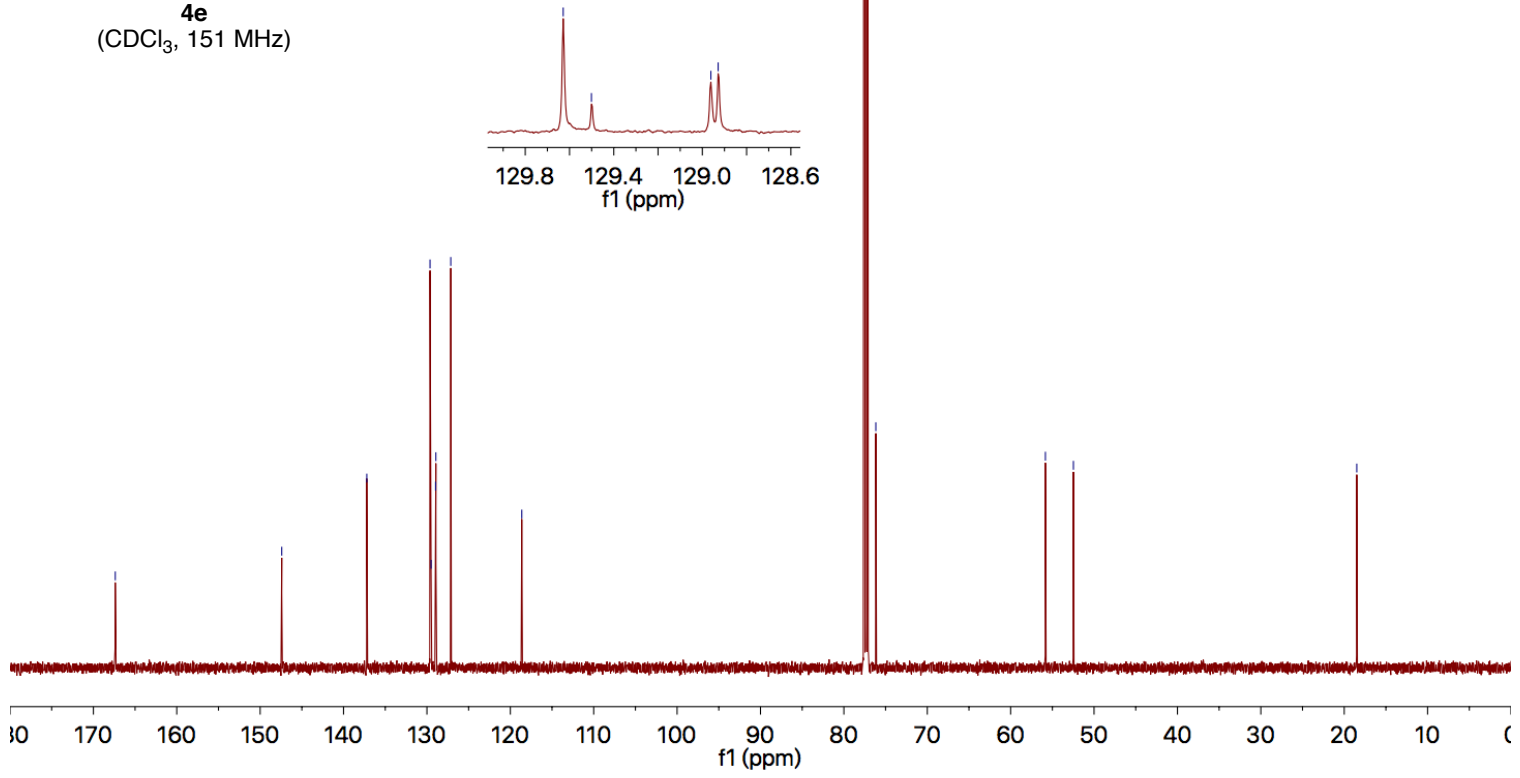

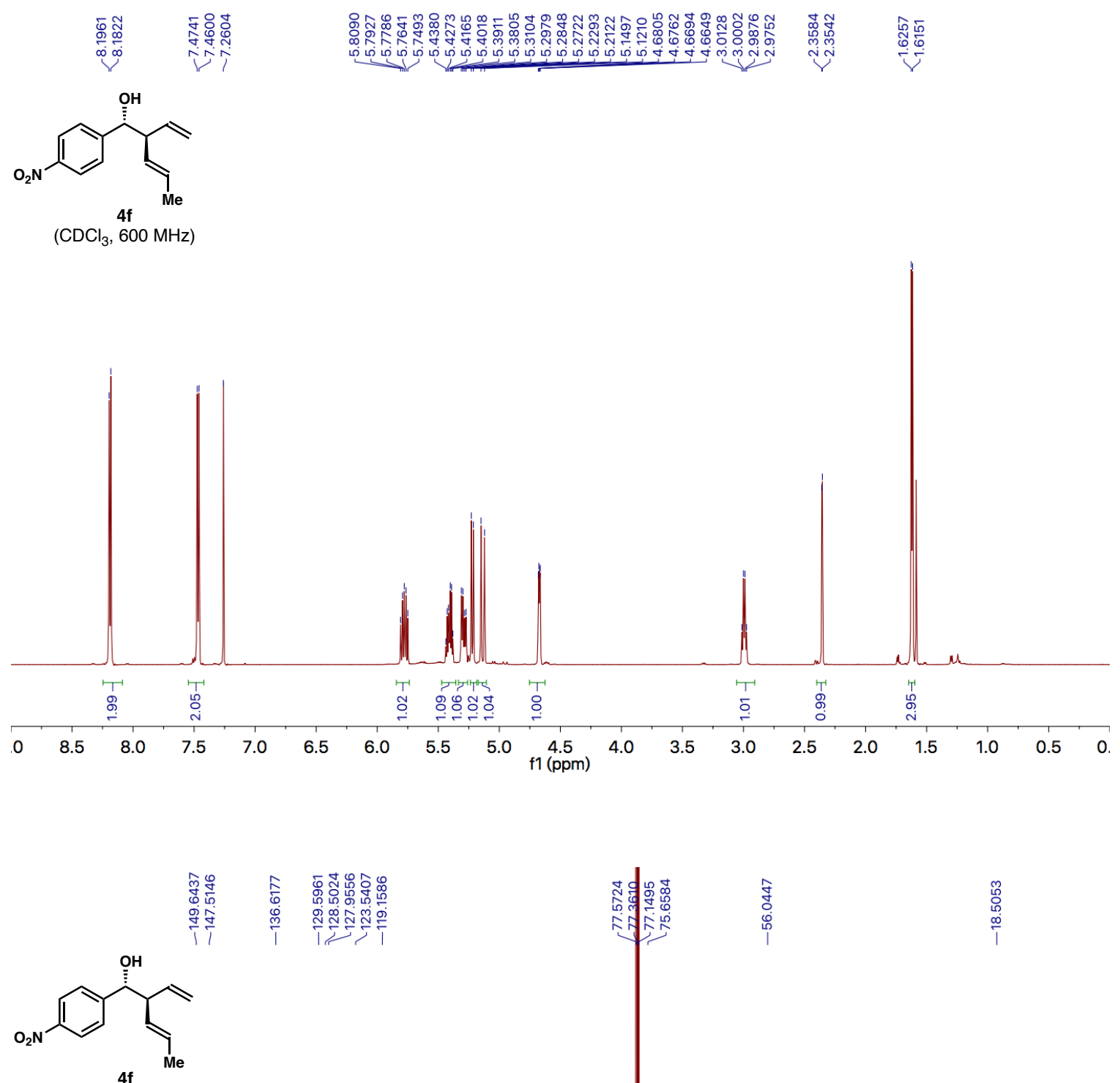

J
i
0

 $\left(\mathrm{CDCl}_{3}, 151 \mathrm{MHz}\right)$

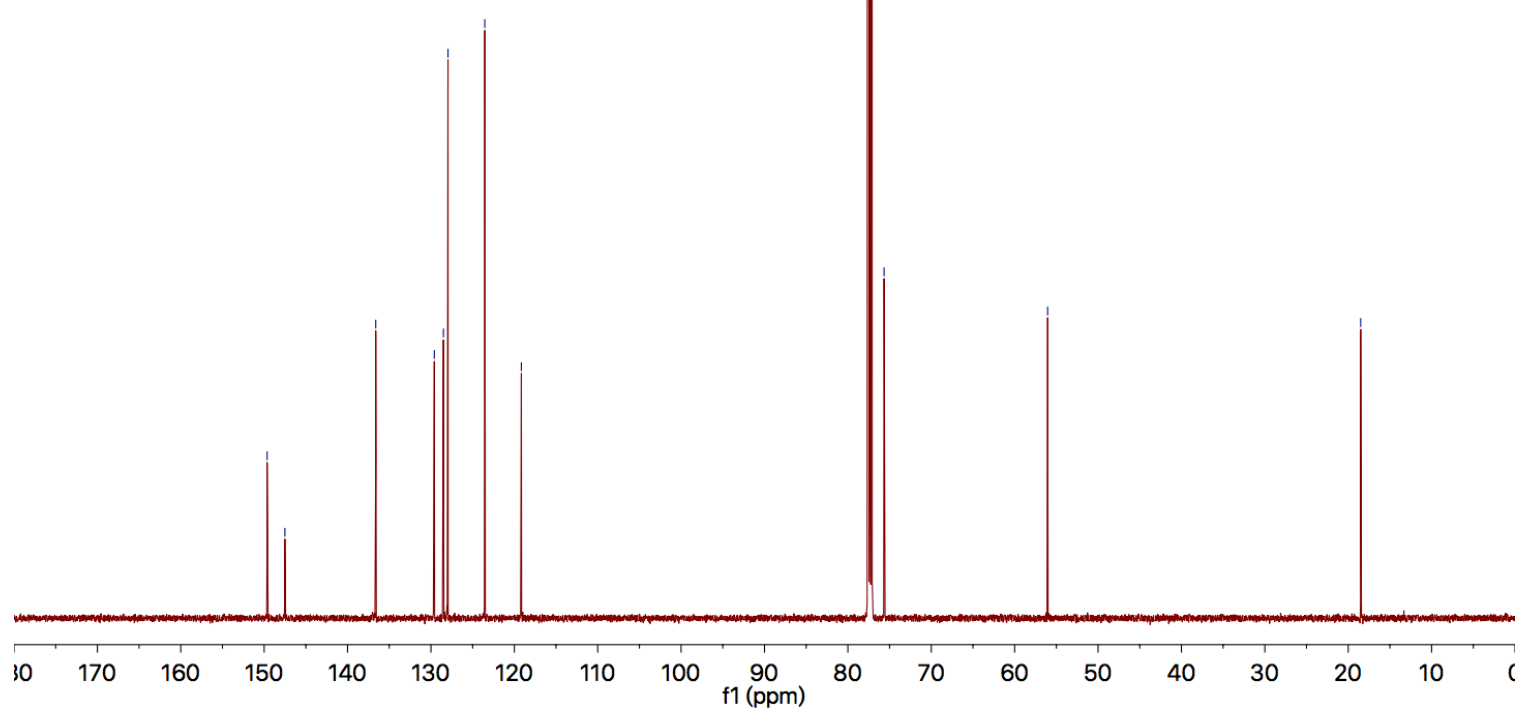



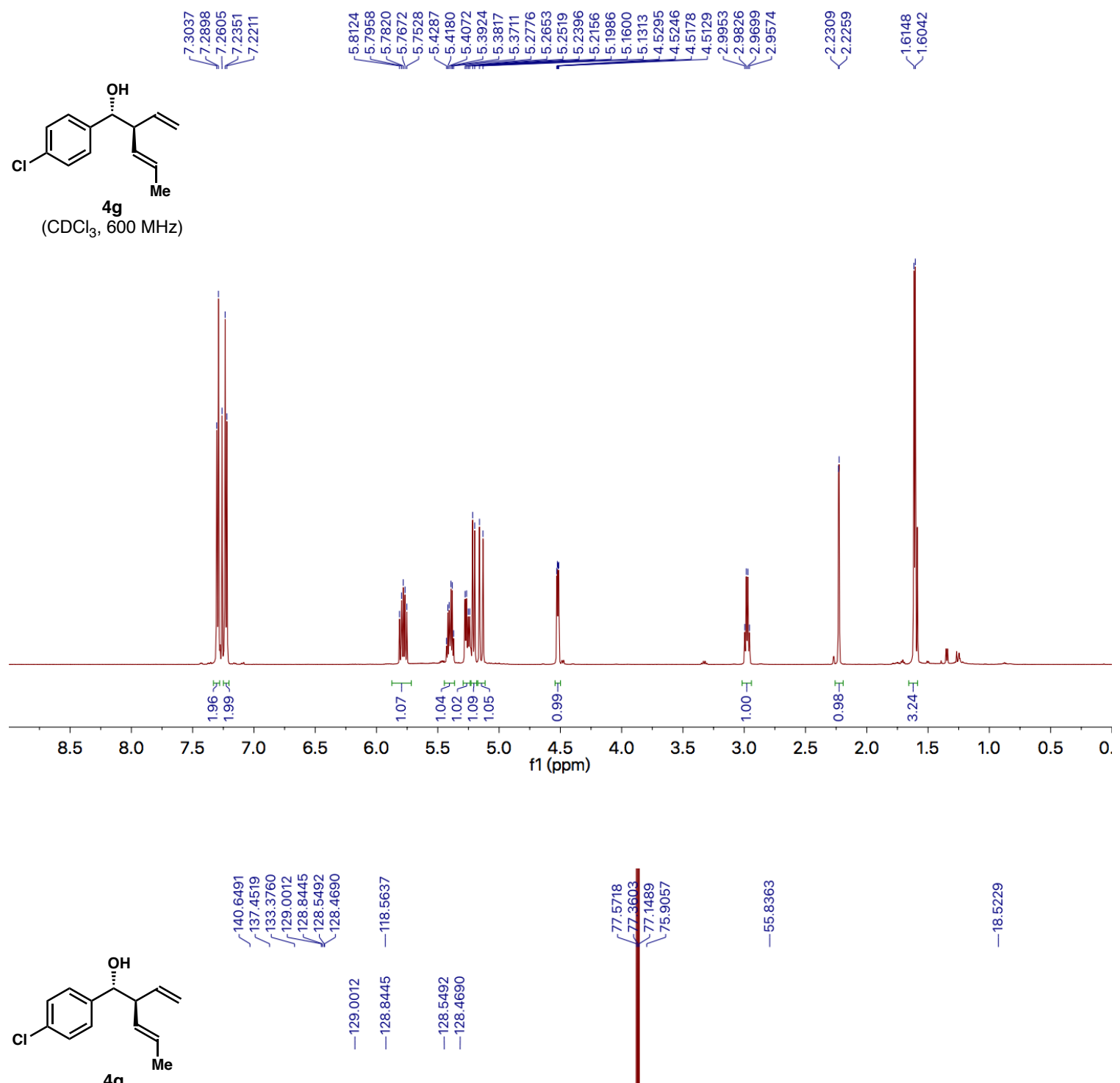

$\left(\mathrm{CDCl}_{3}, 151 \mathrm{MHz}\right)$
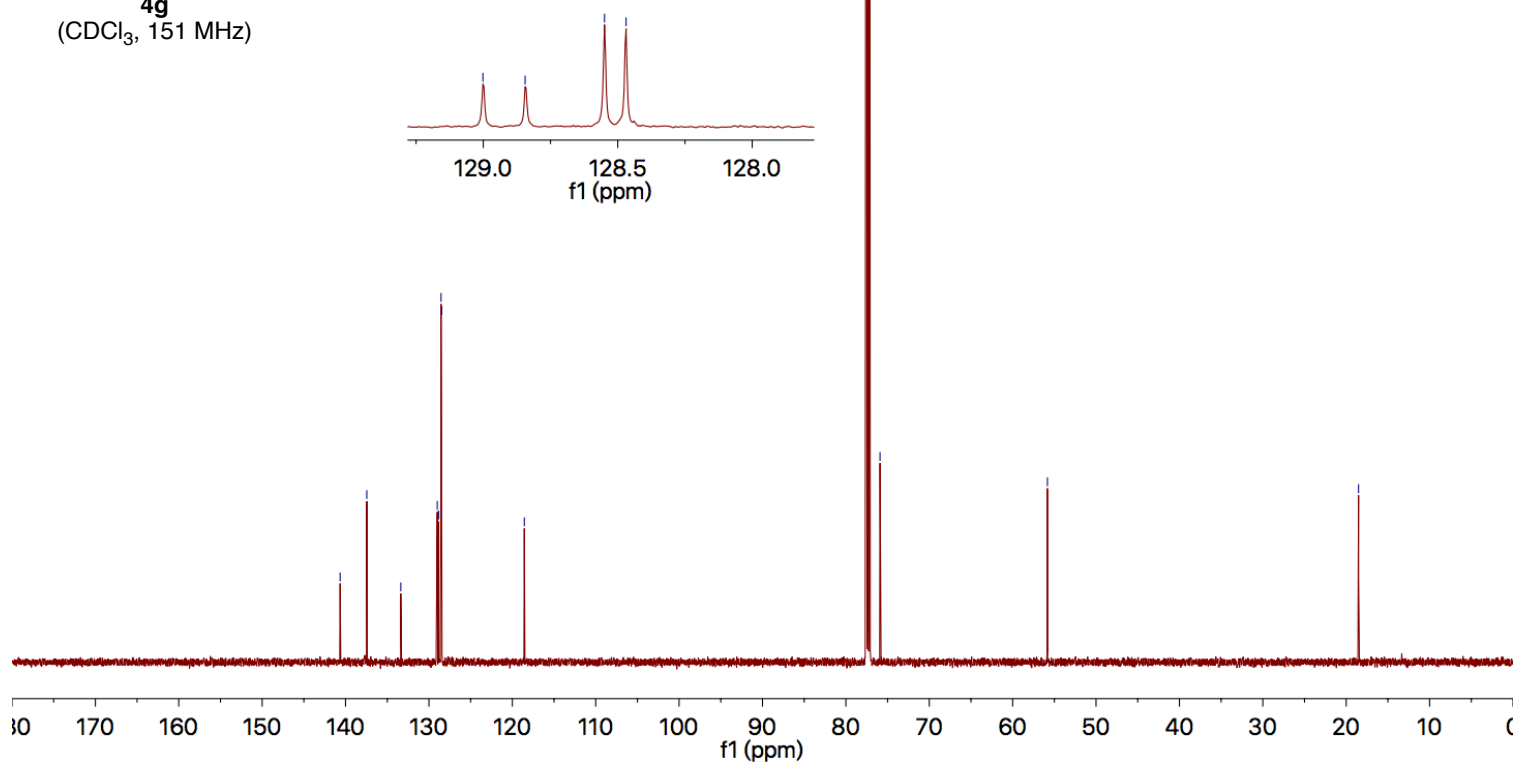

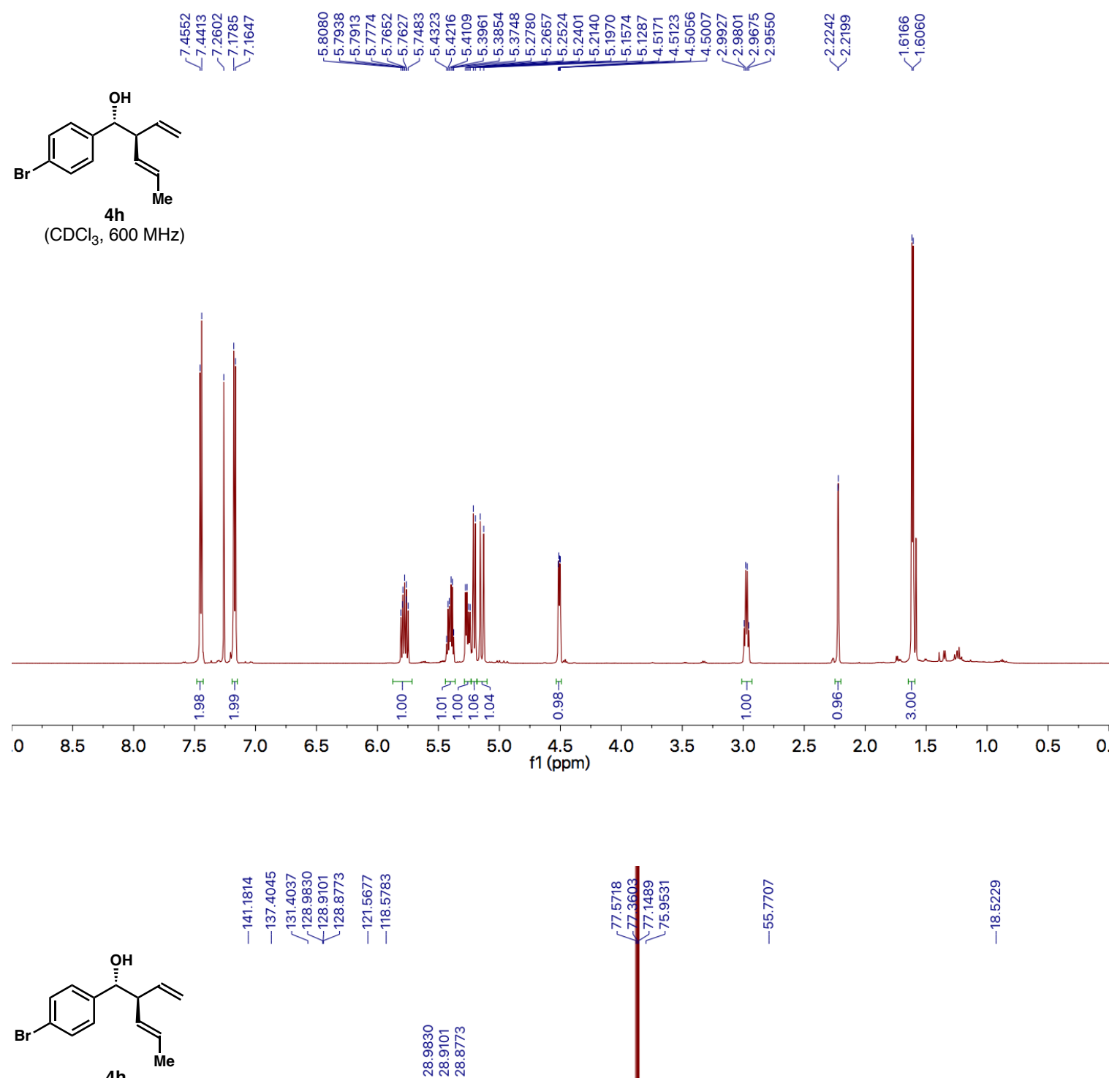

$\left(\mathrm{CDCl}_{3}, 151 \mathrm{MHz}\right)$
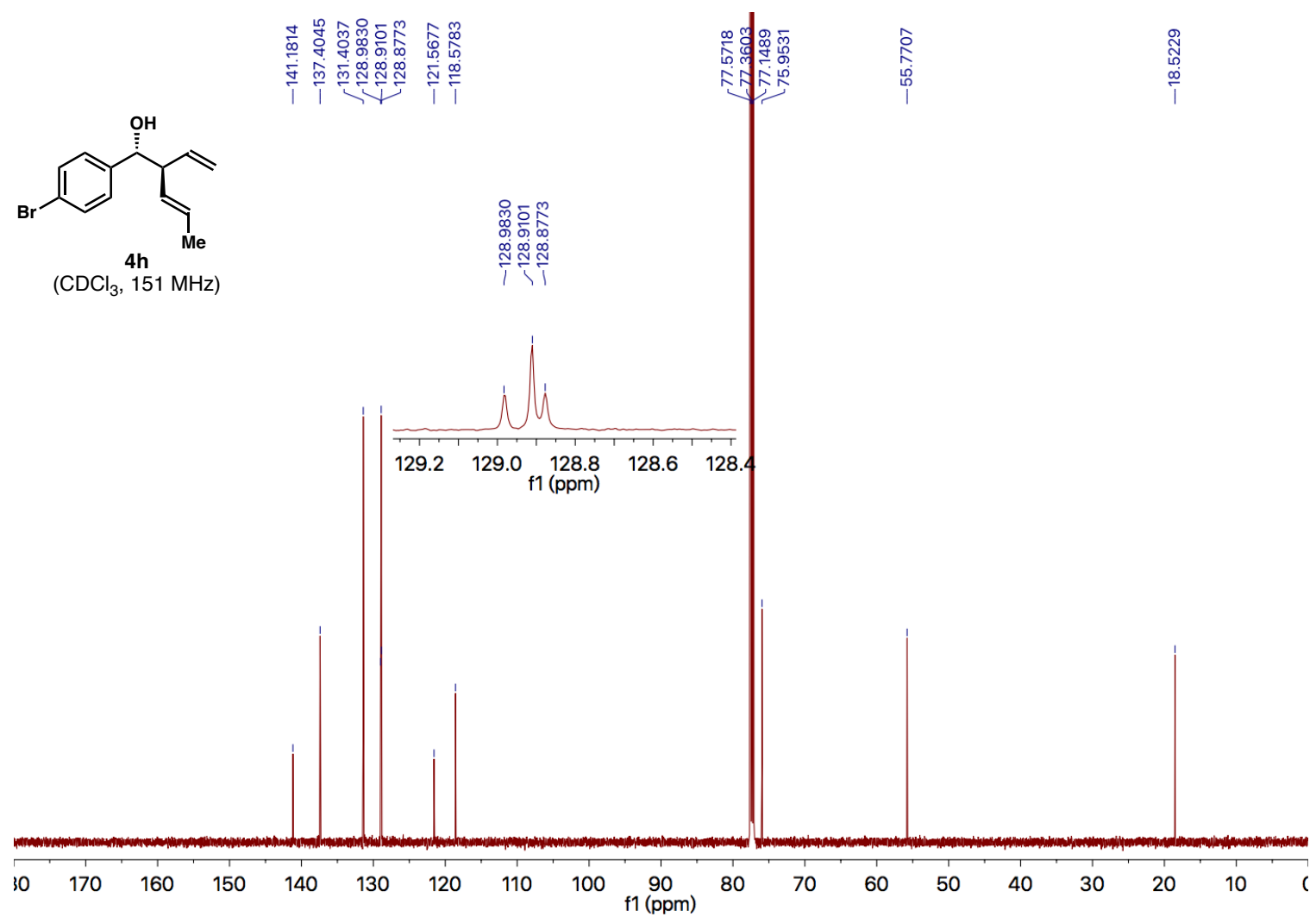

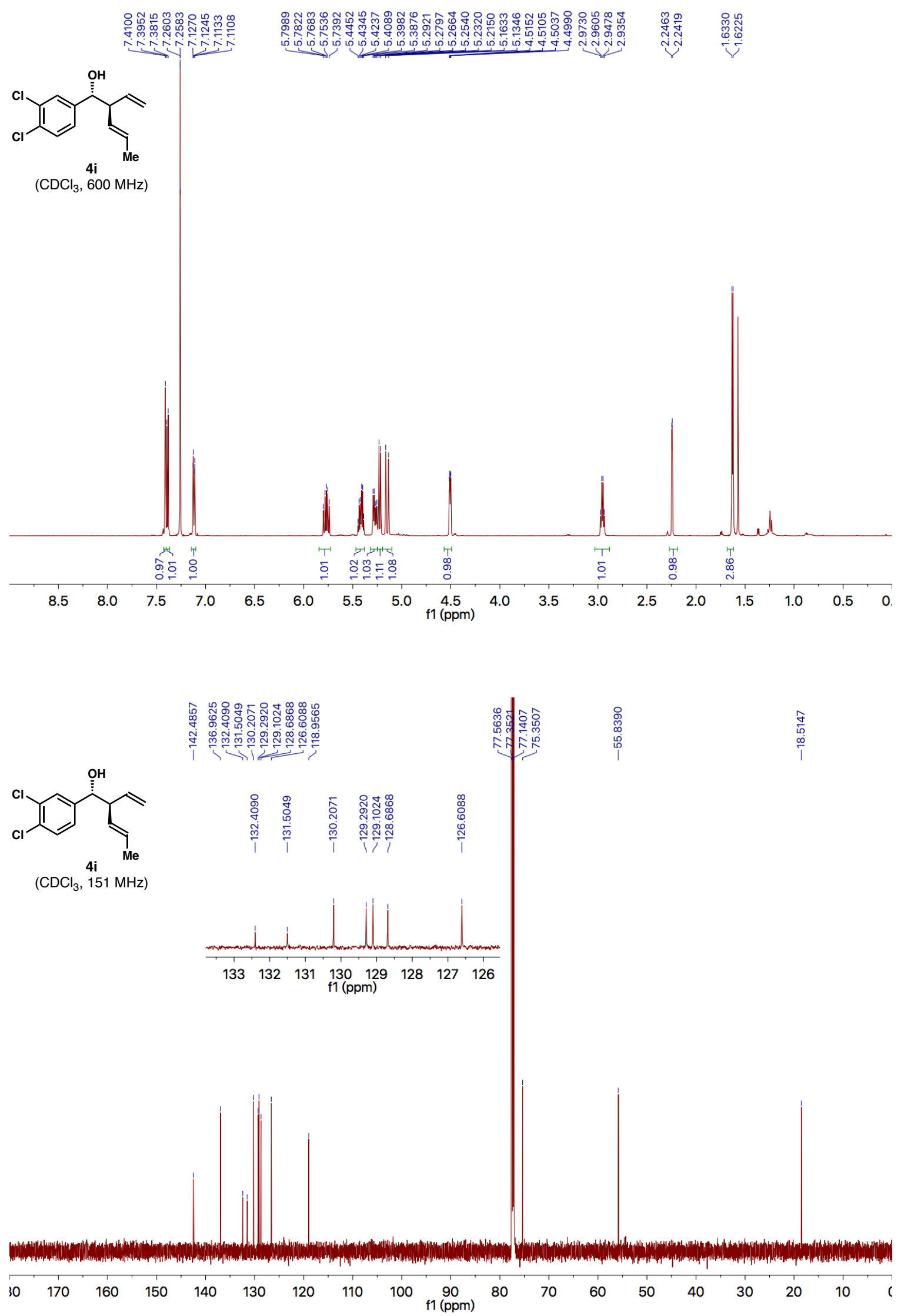

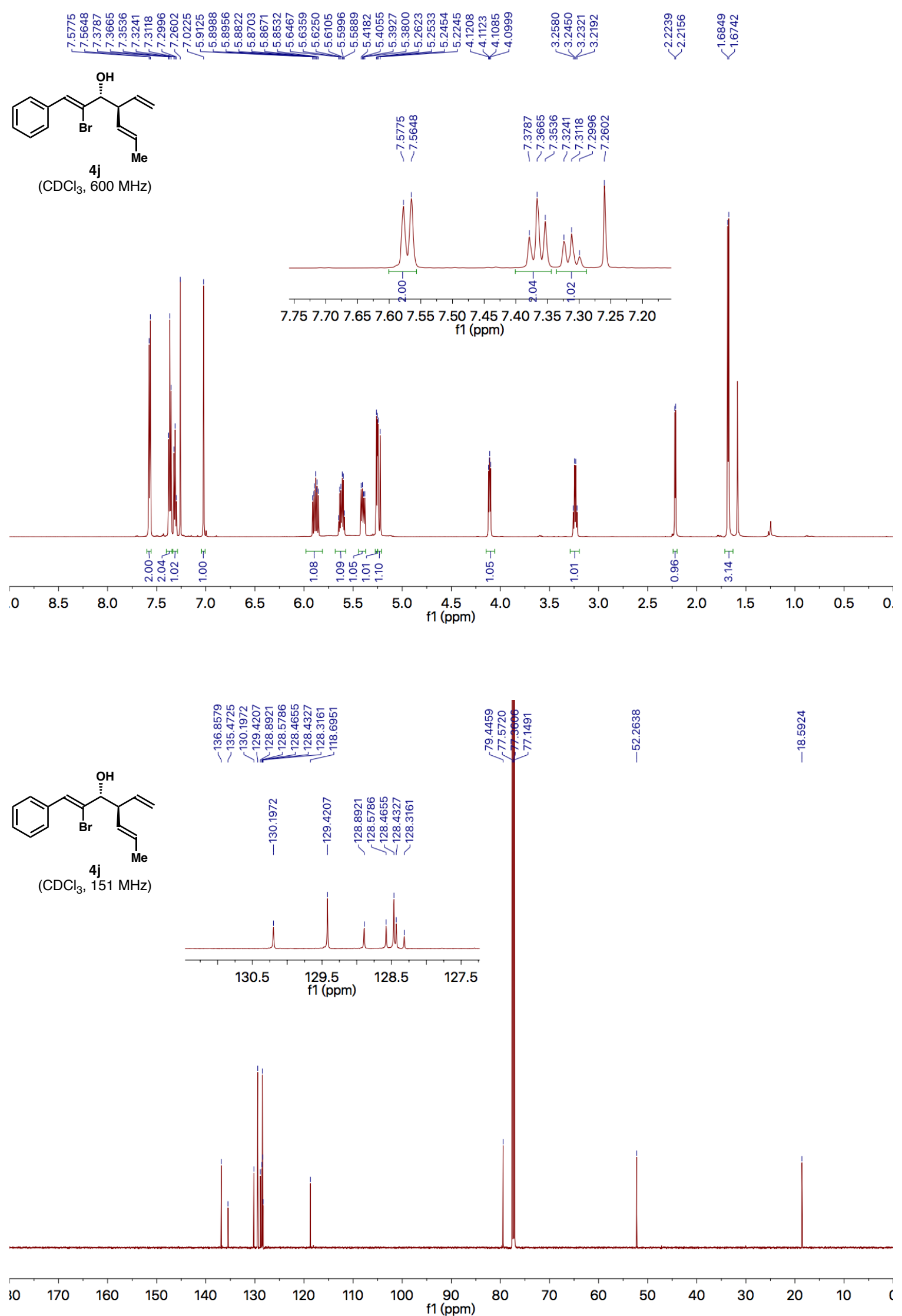

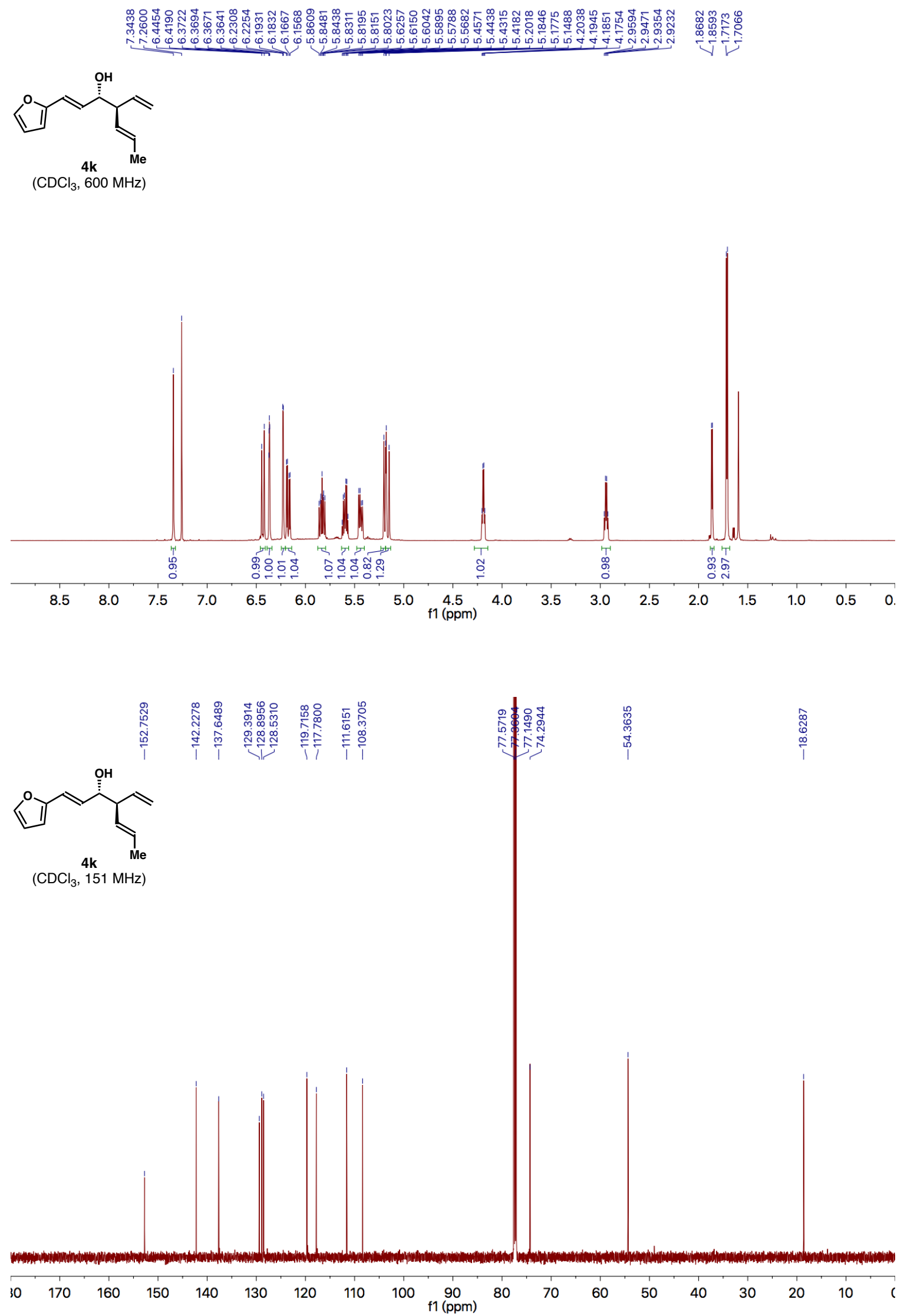

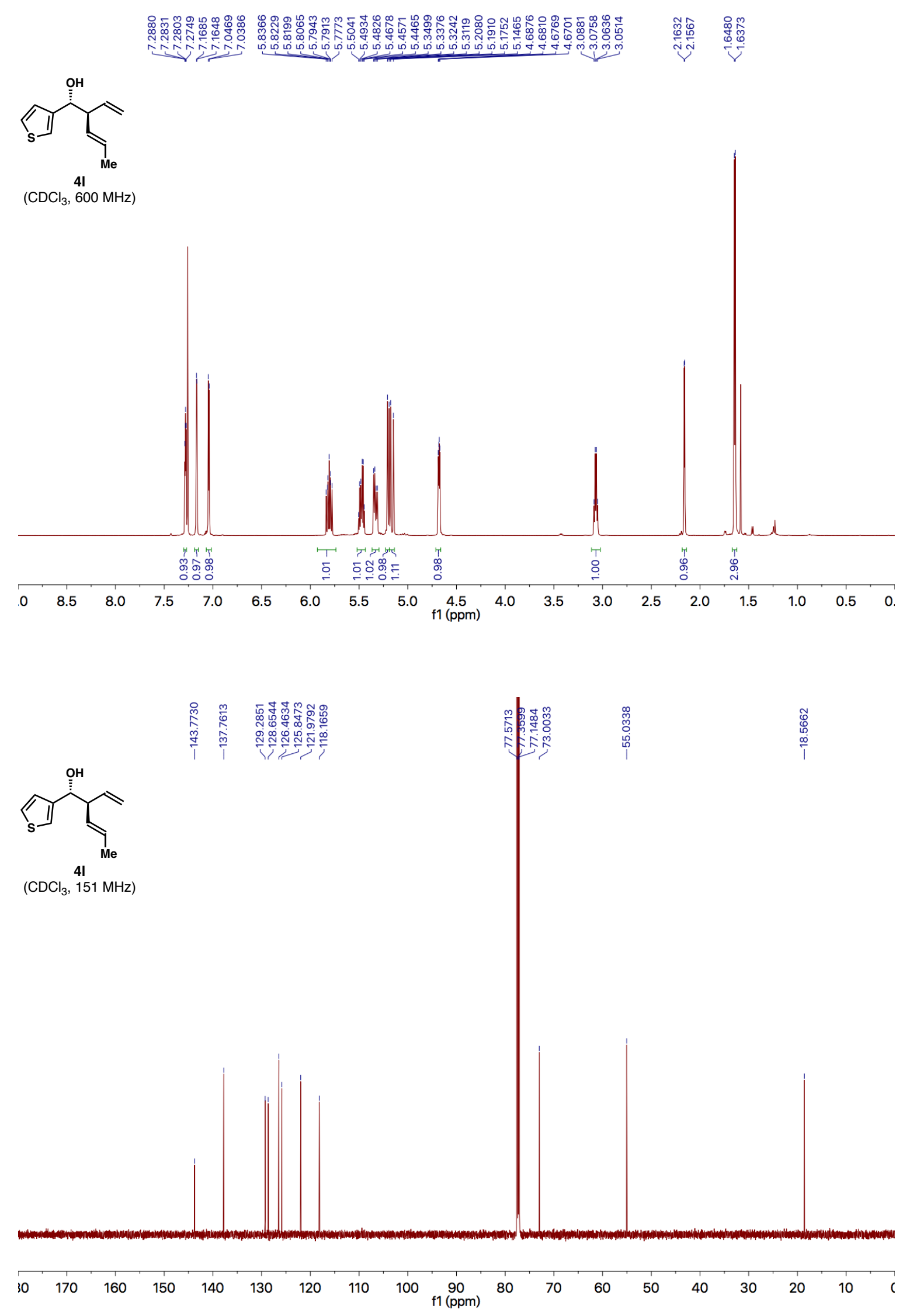

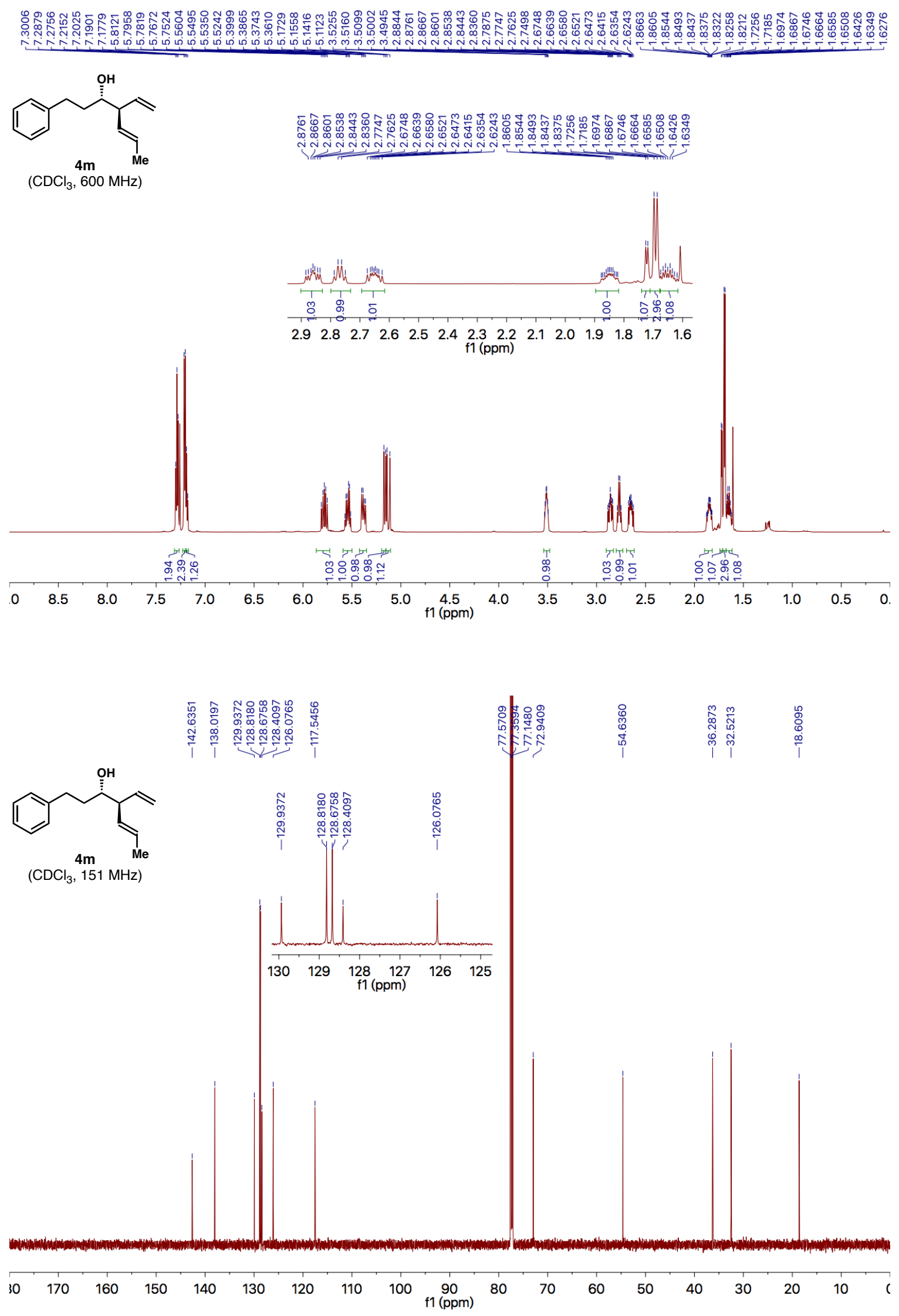

SI-74 

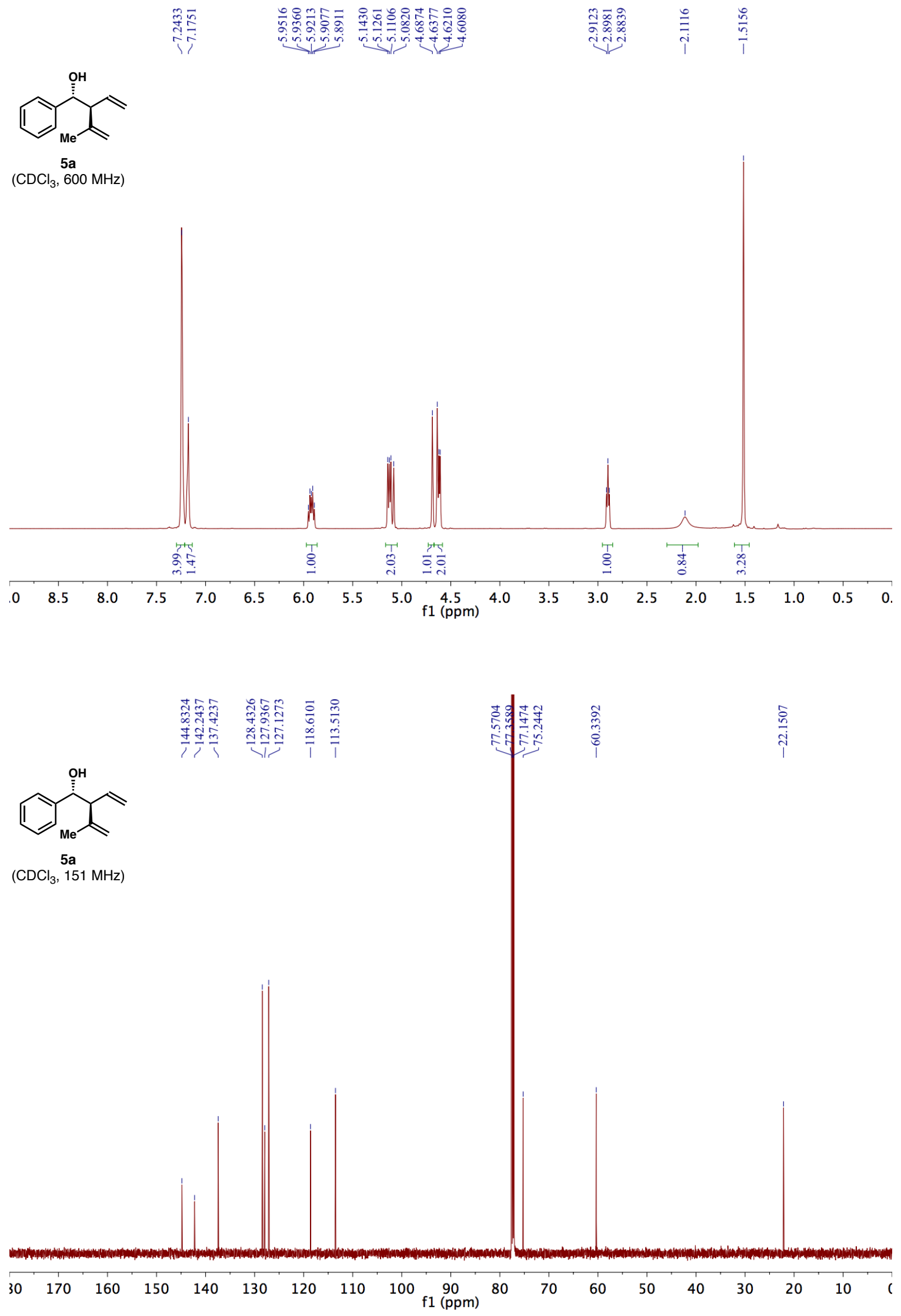

SI-75 

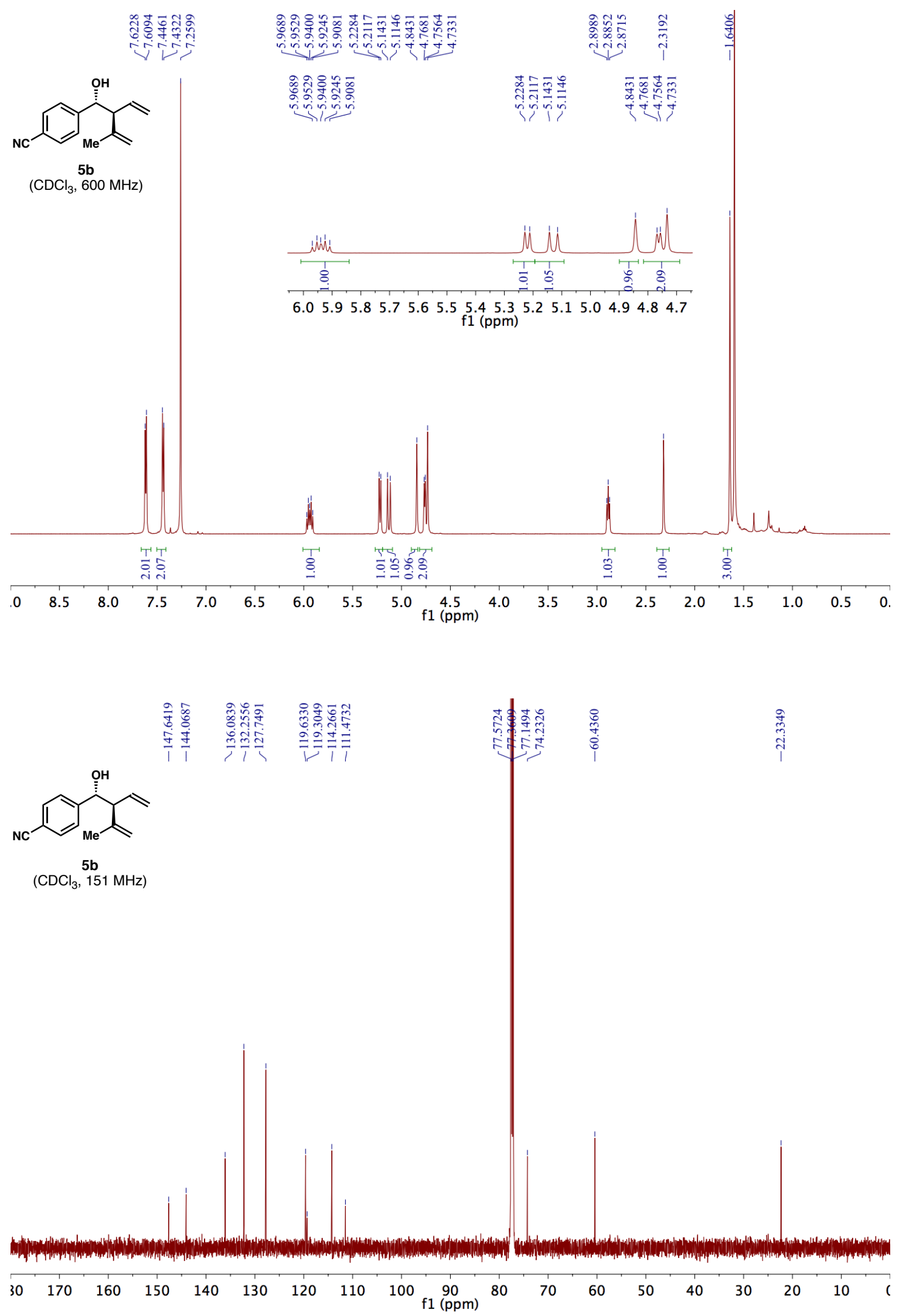

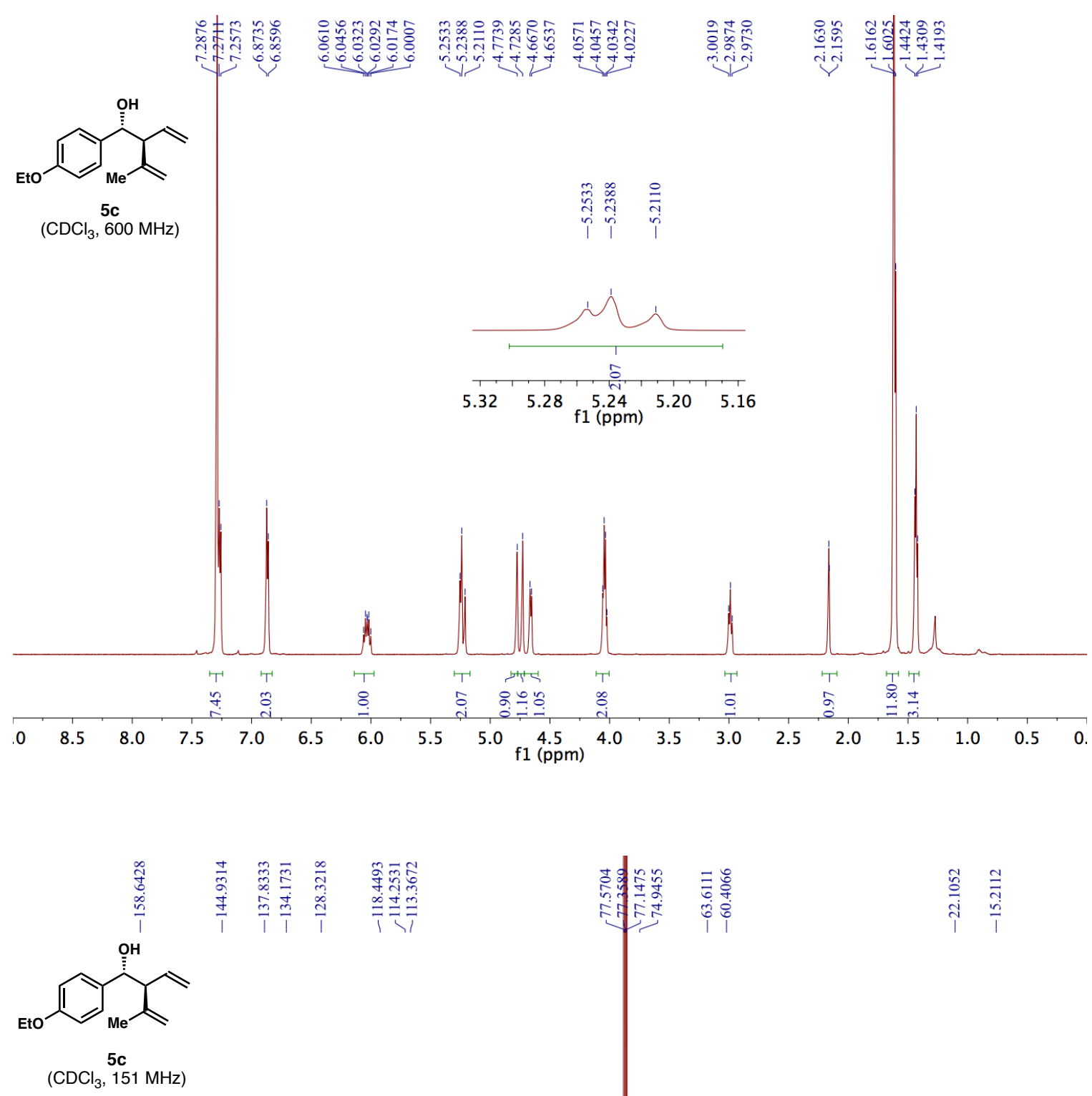

กิ

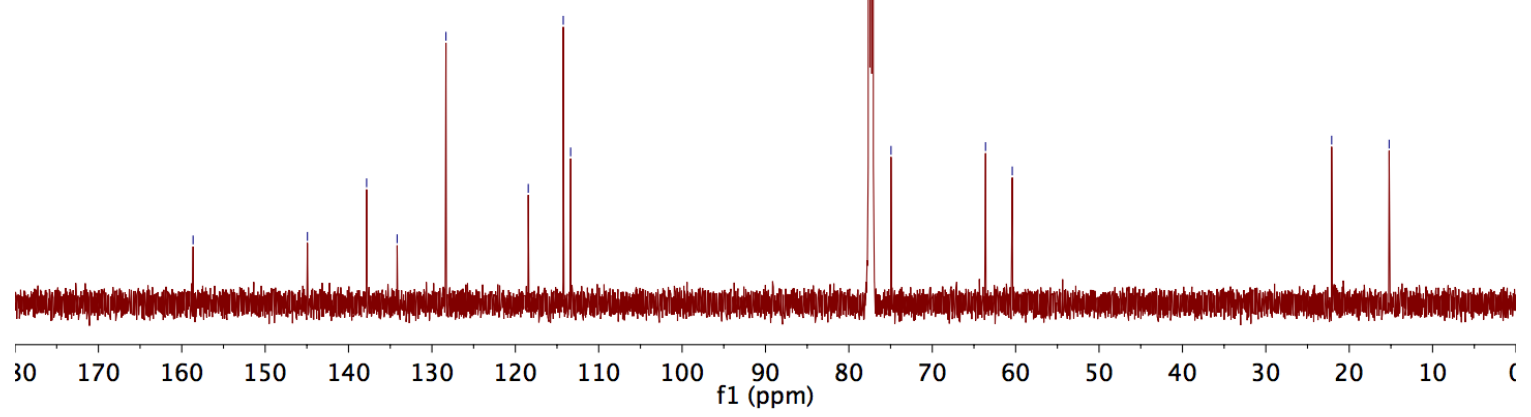



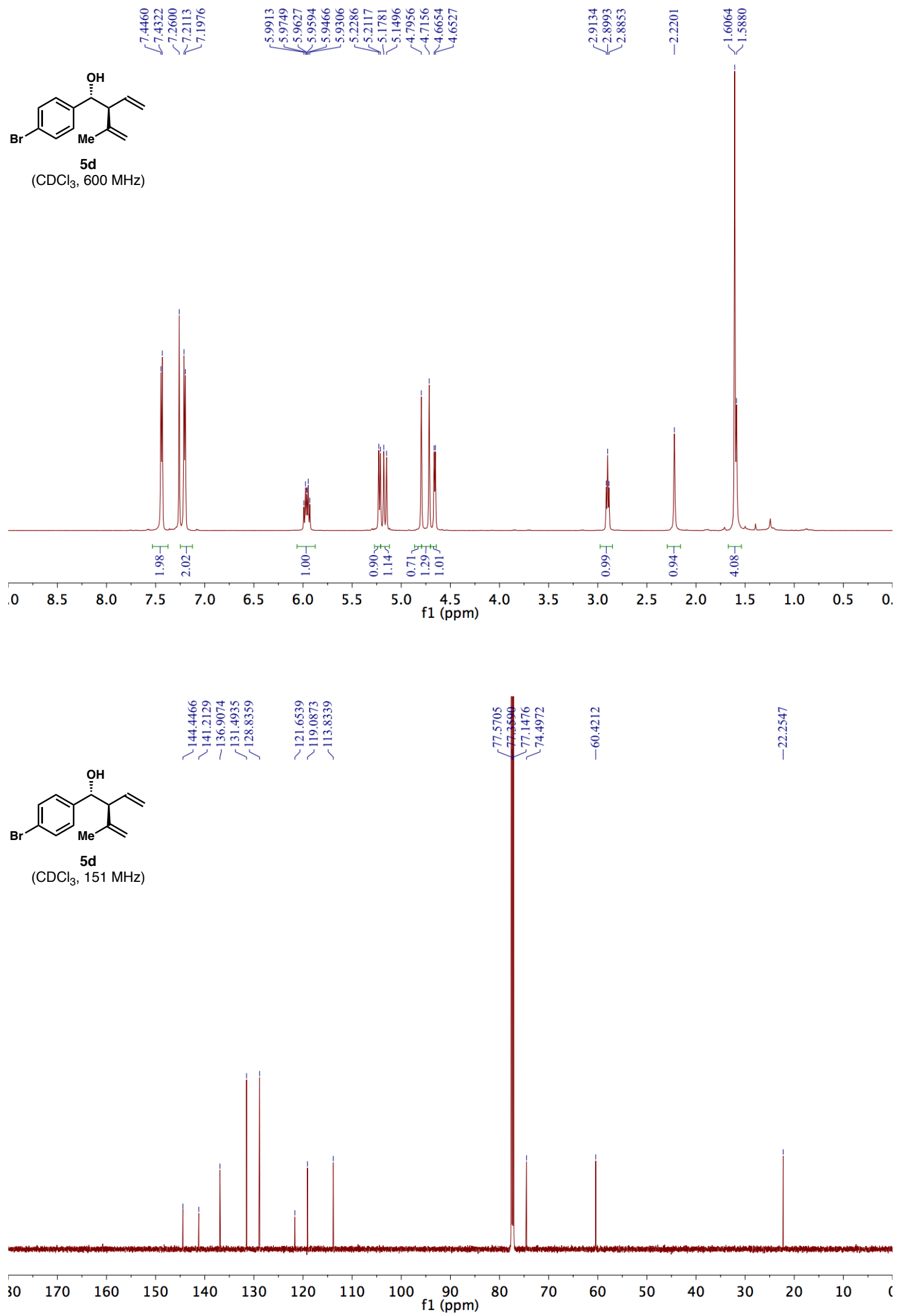

SI-78 

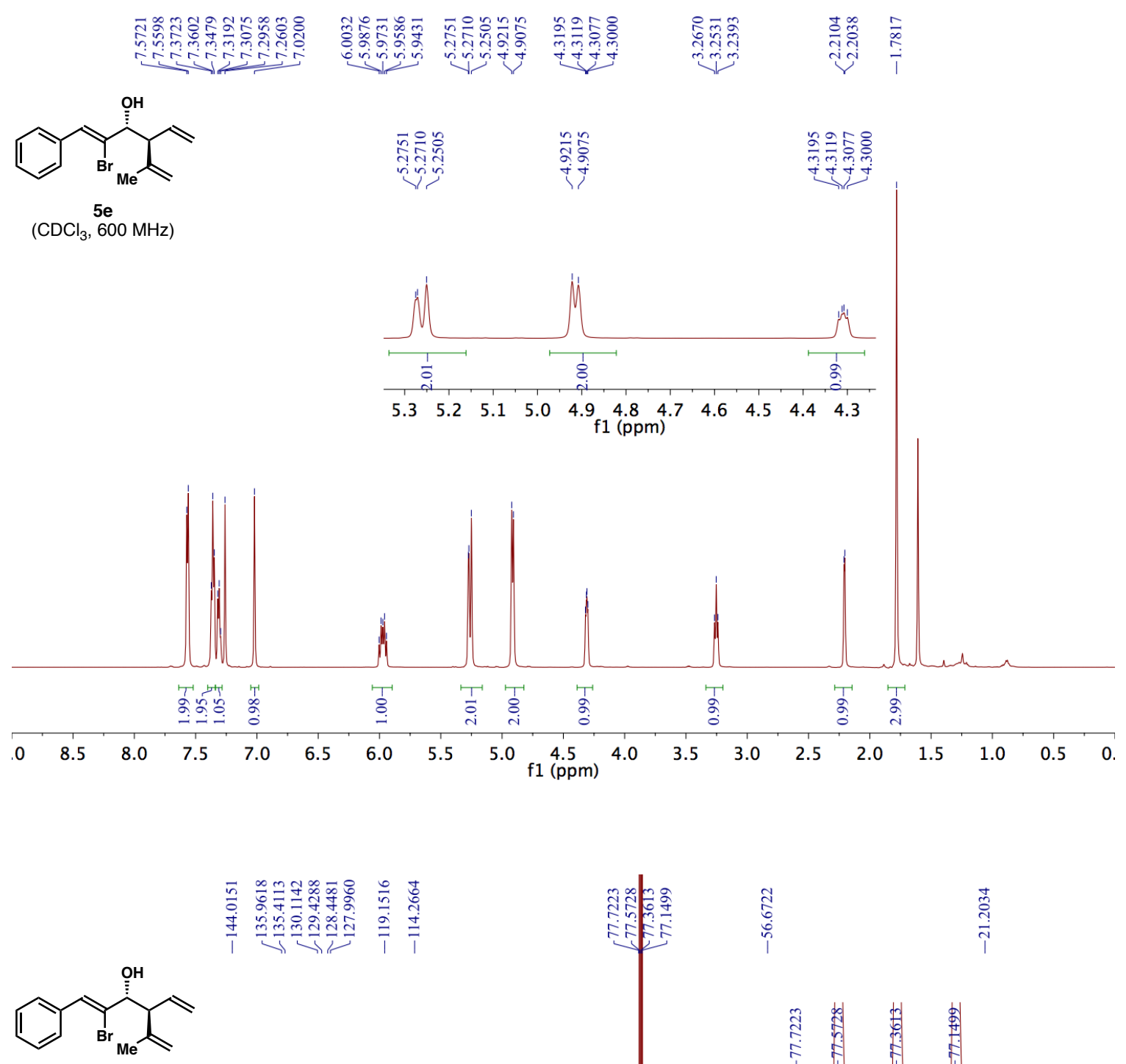

$\mathbf{5 e}$
$\left(\mathrm{CDCl}_{3}, 151 \mathrm{MHz}\right)$

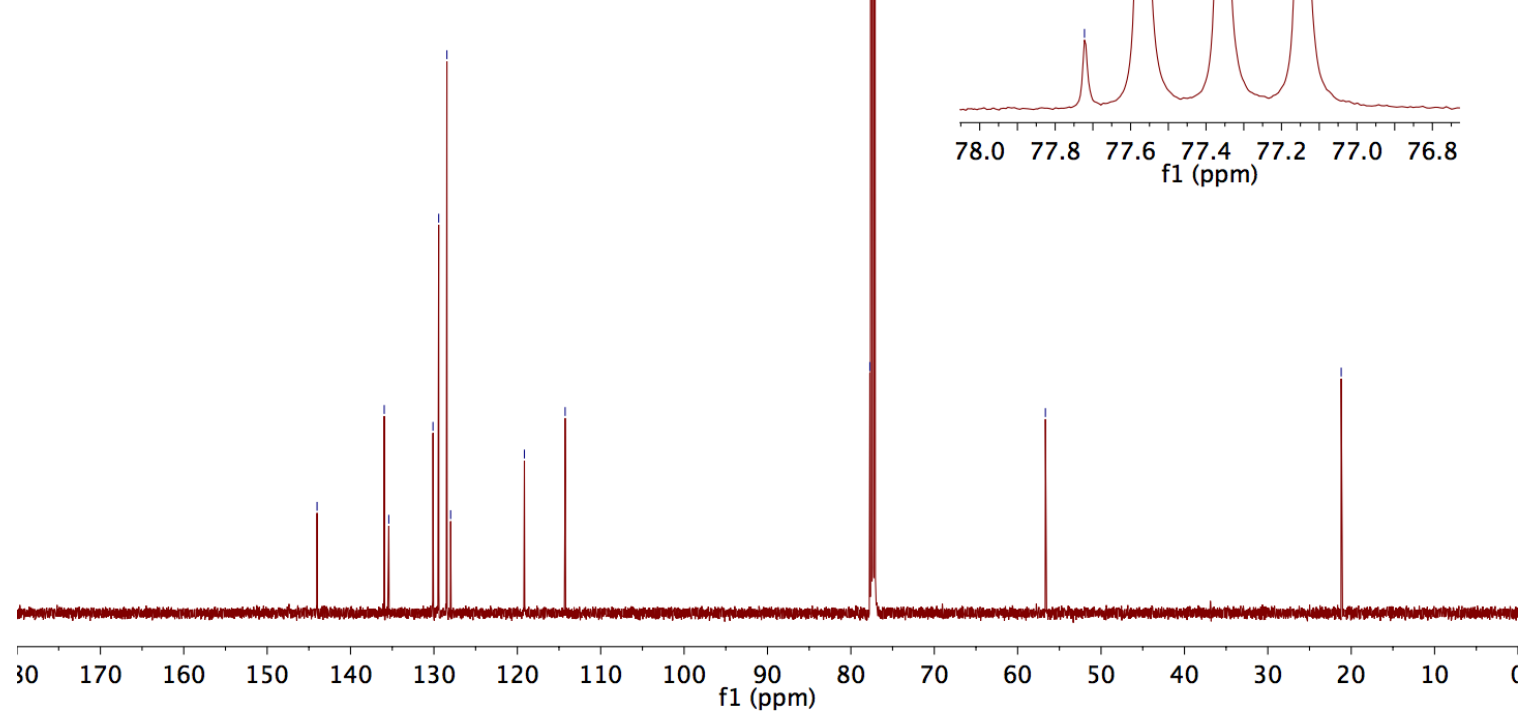

SI-79 

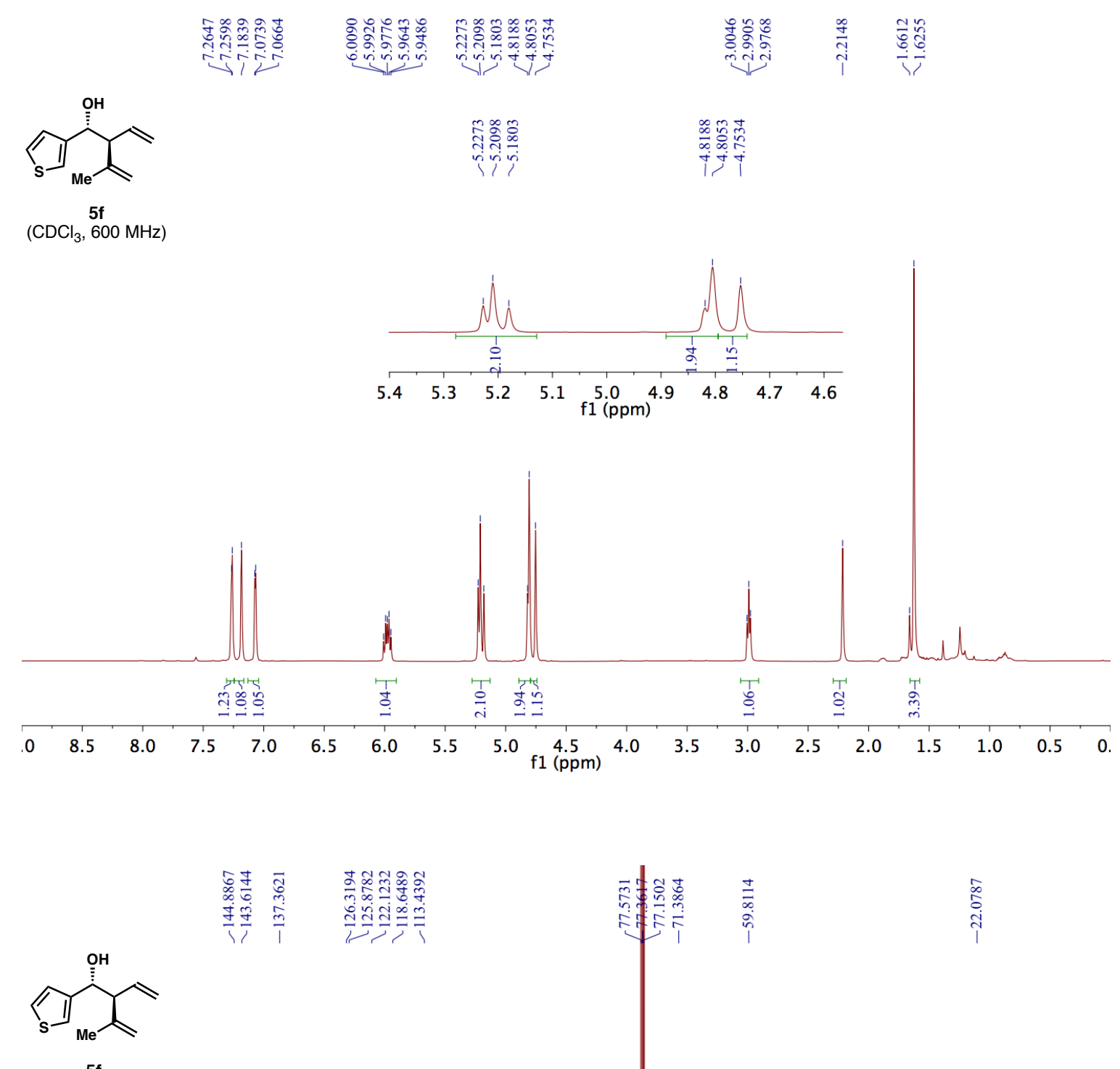

$\left(\mathrm{CDCl}_{3}, 151 \mathrm{MHz}\right)$

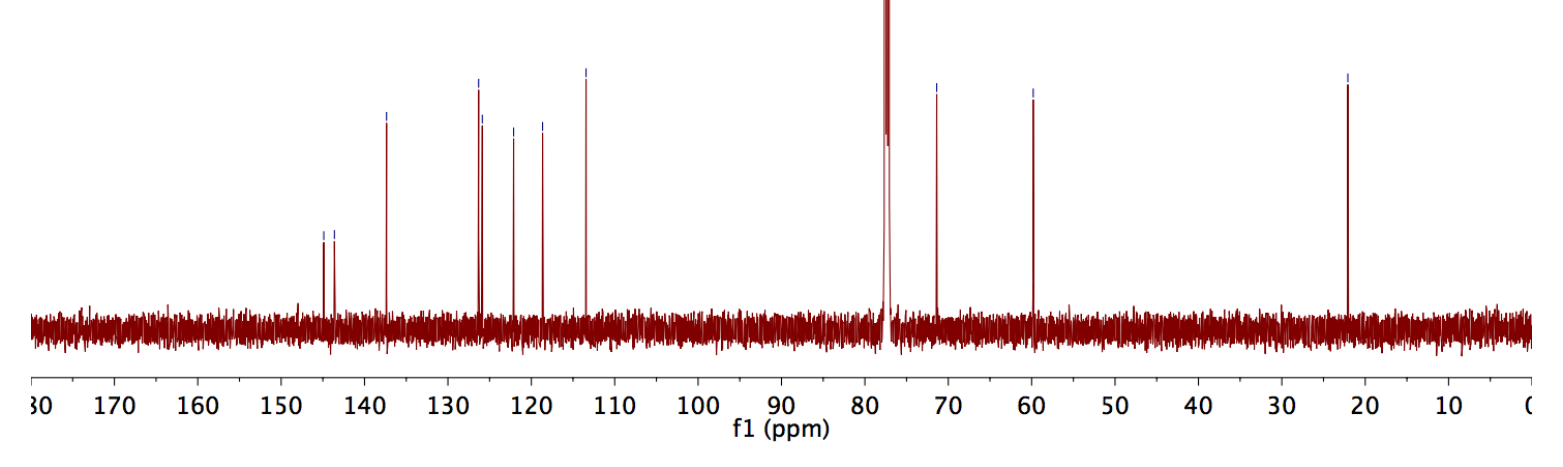



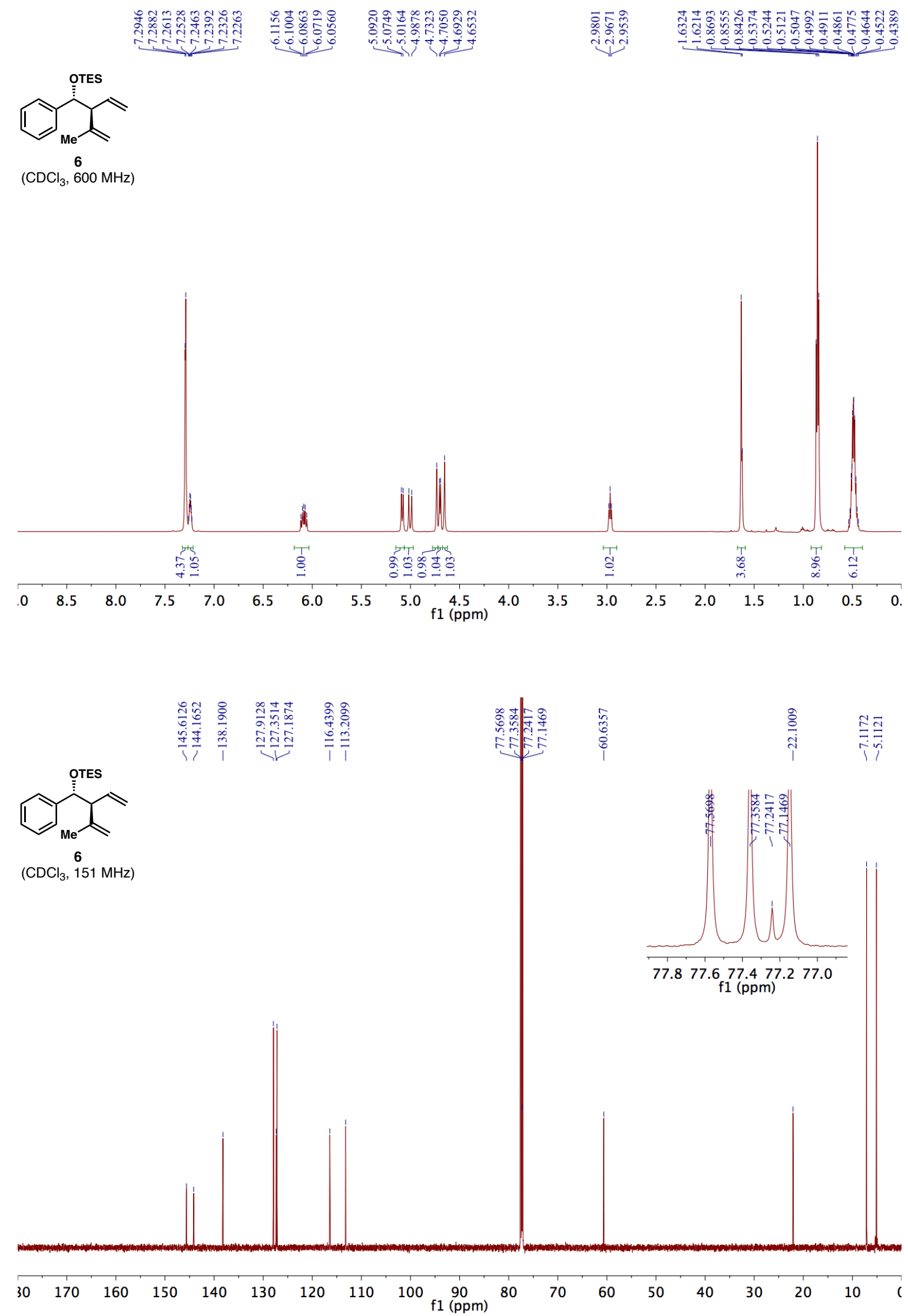


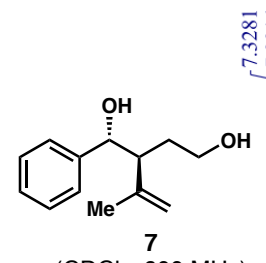

$\left(\mathrm{CDCl}_{3}, 600 \mathrm{MHz}\right)$

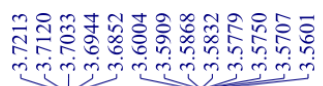

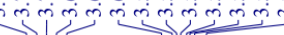

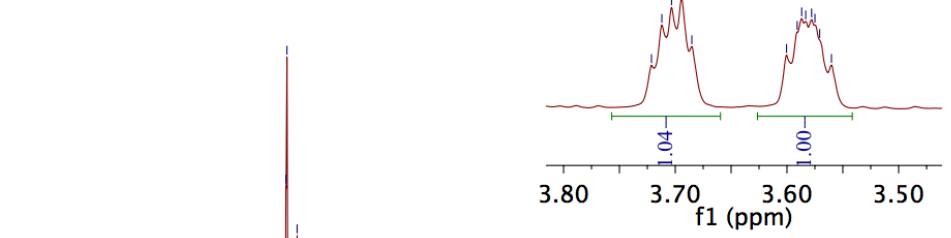

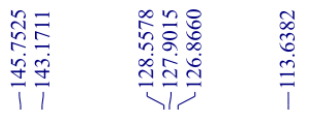

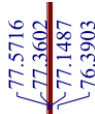

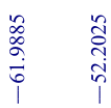

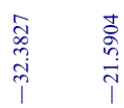

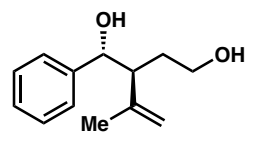

$\left(\mathrm{CDCl}_{3}, 151 \mathrm{MHz}\right)$

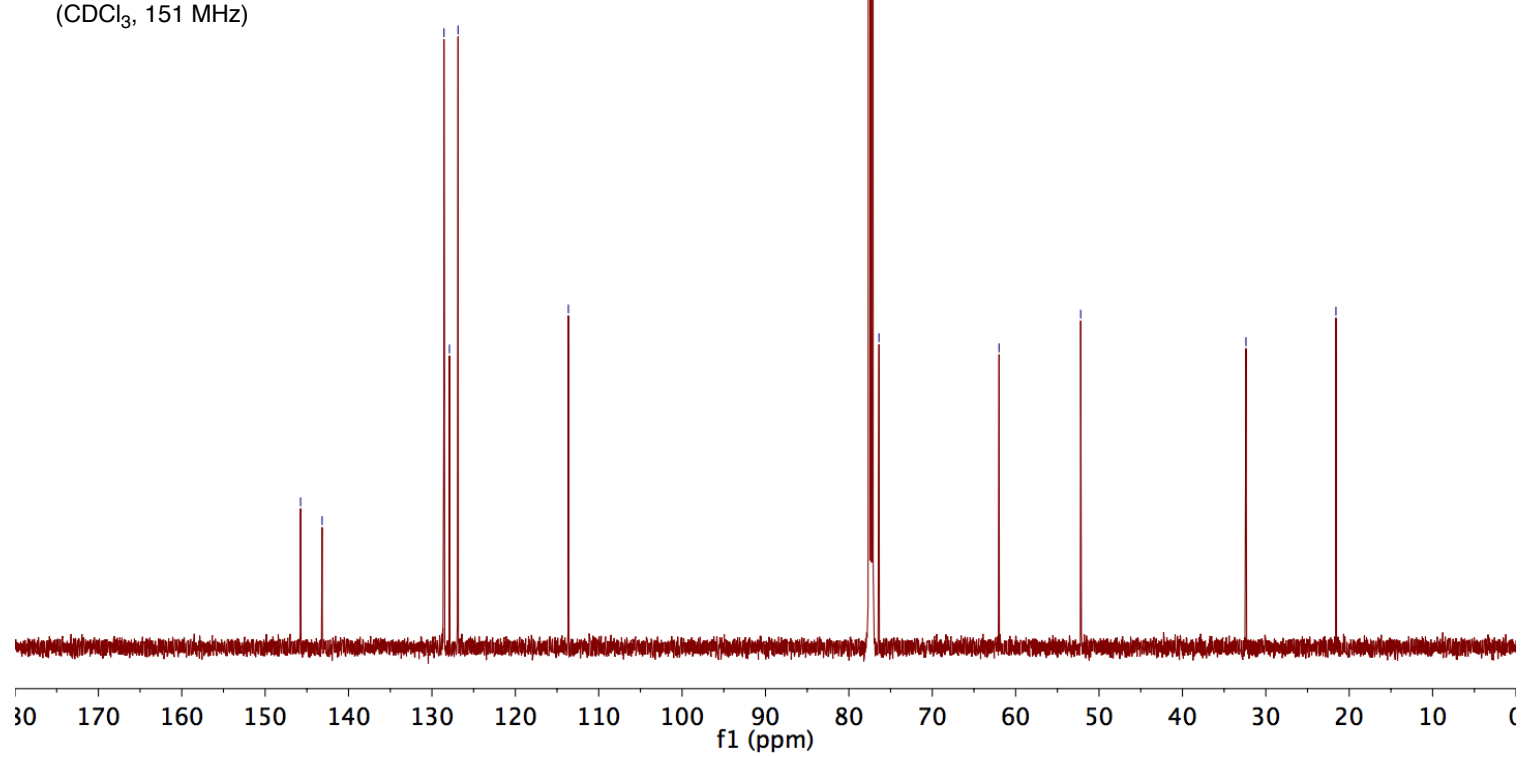



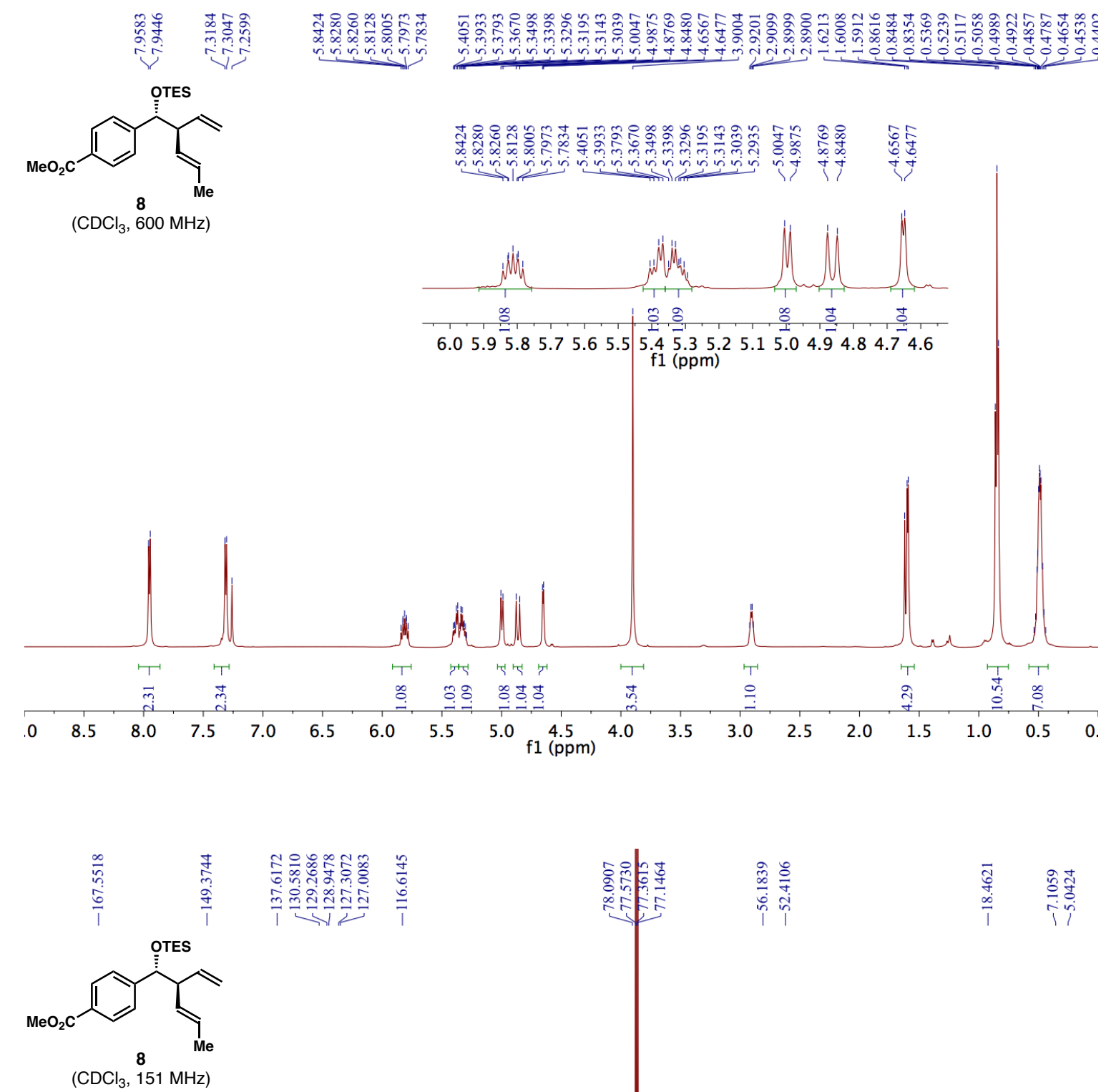

के
$\infty$
0
0
0
0
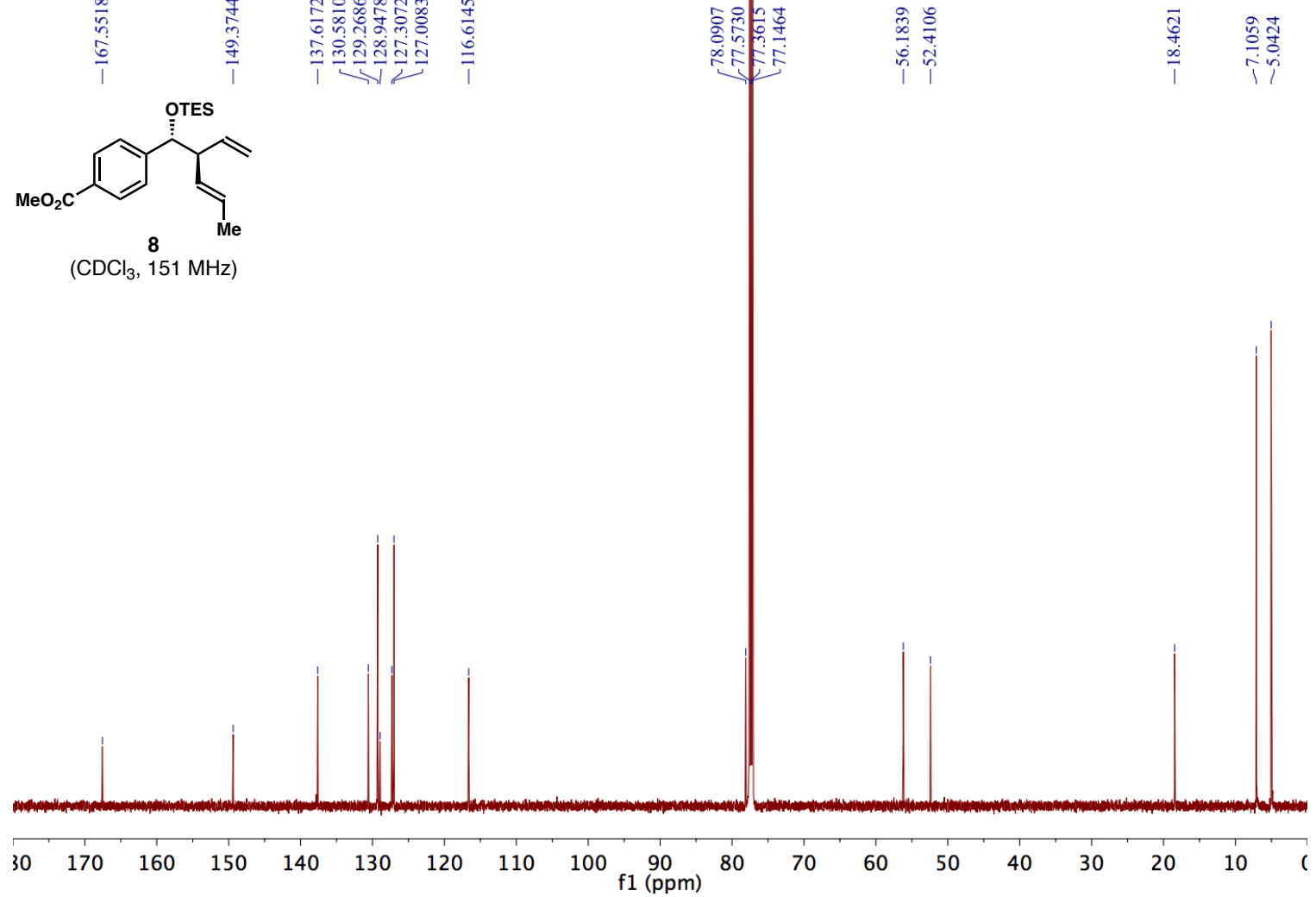

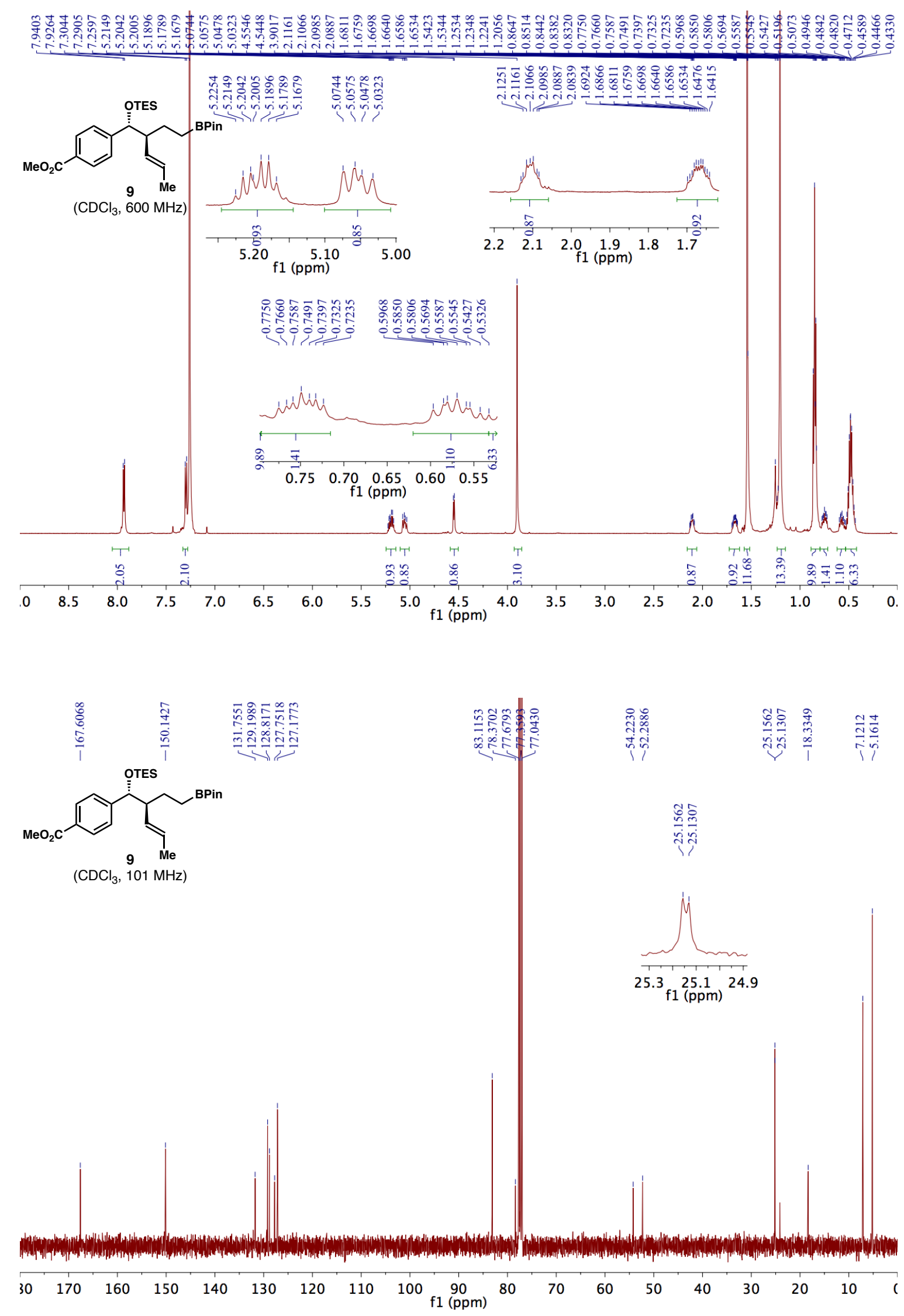

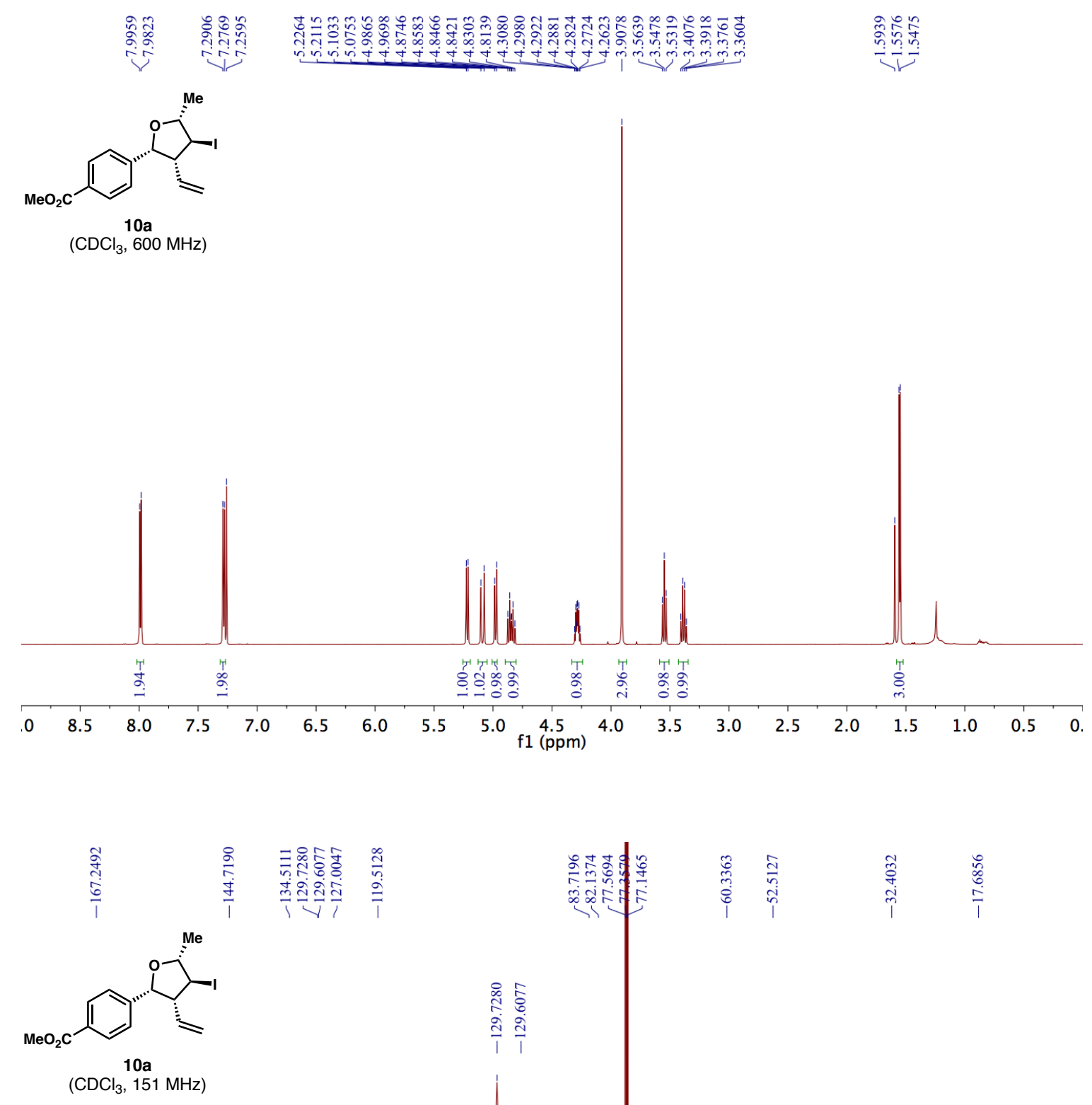

ำ

ปิ
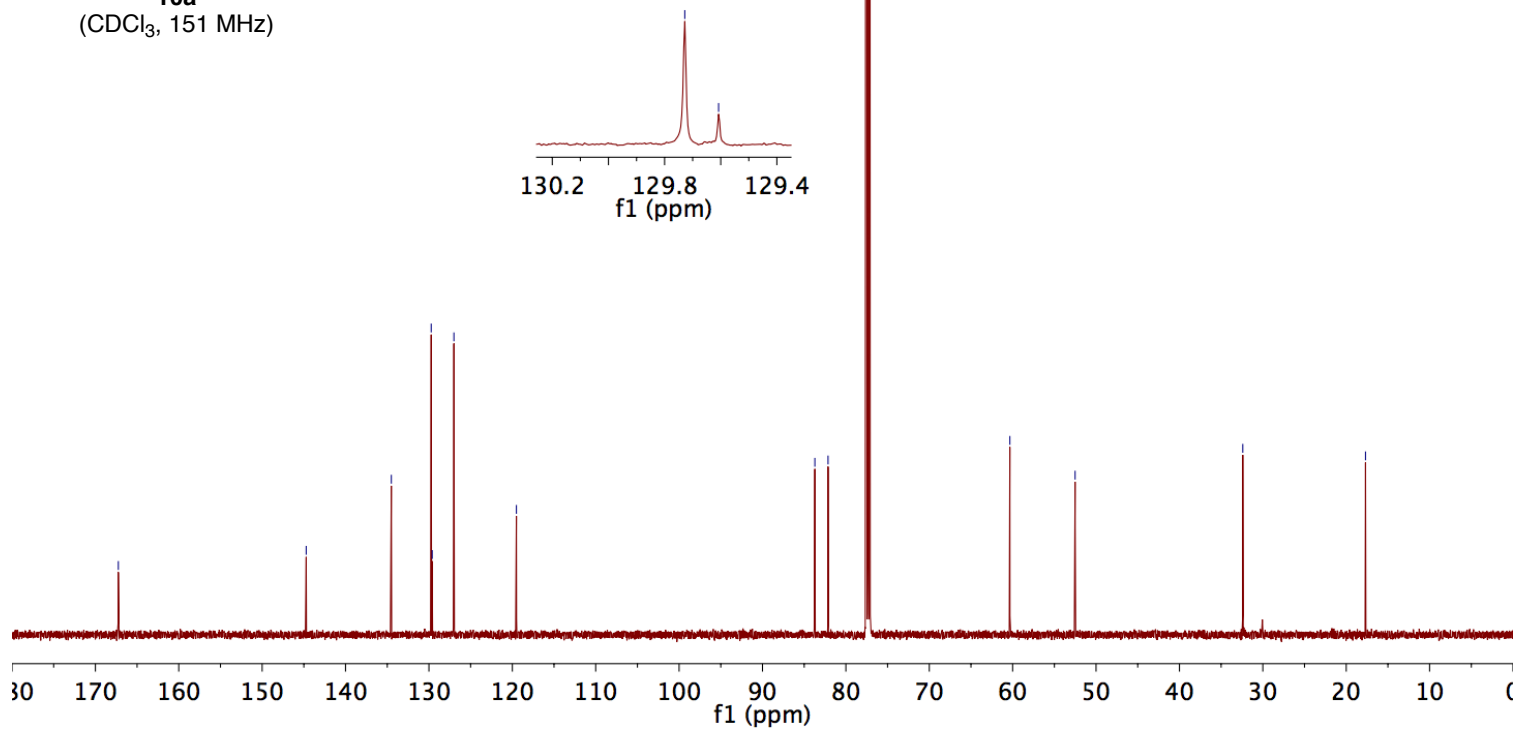

SI-85 

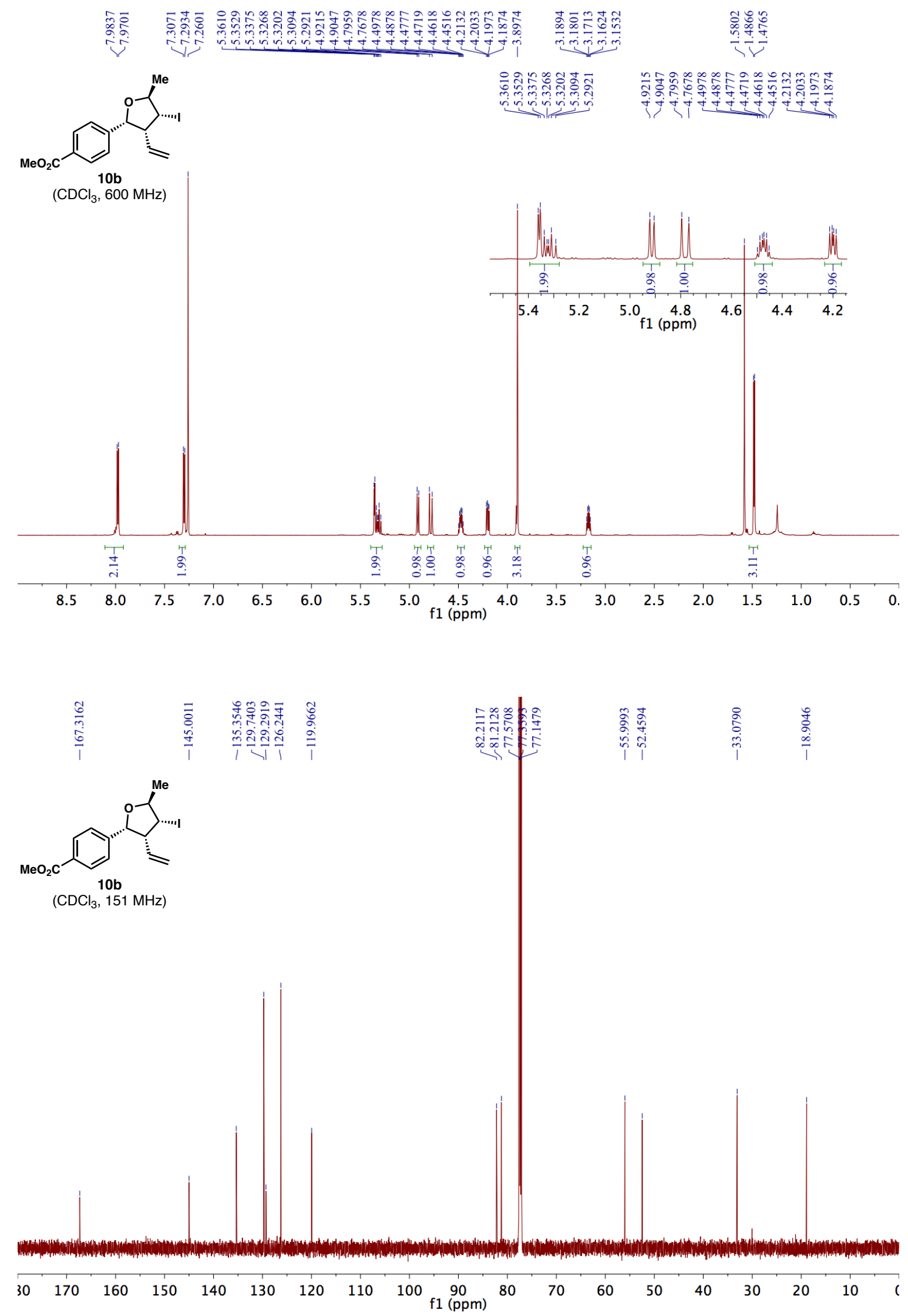

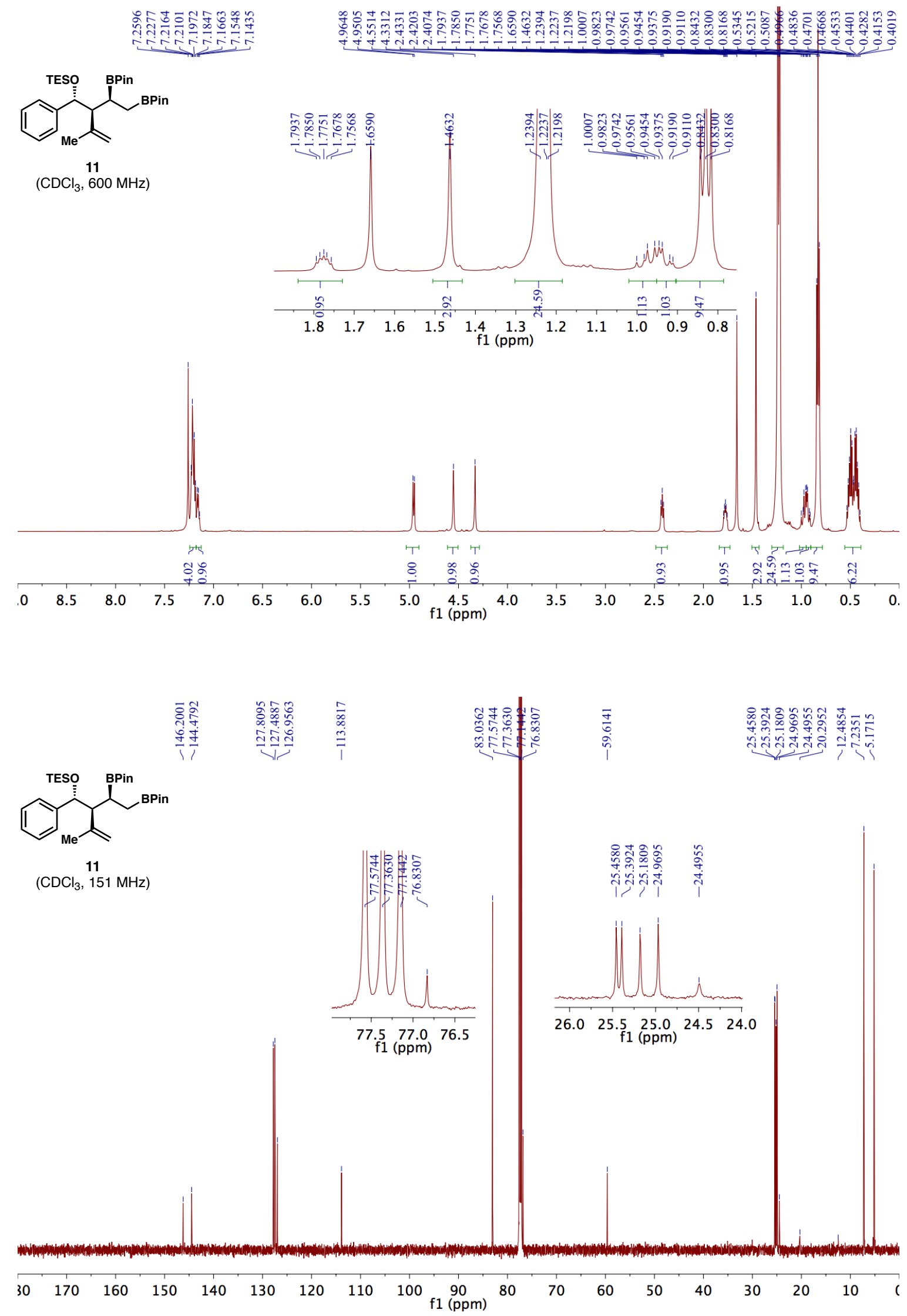

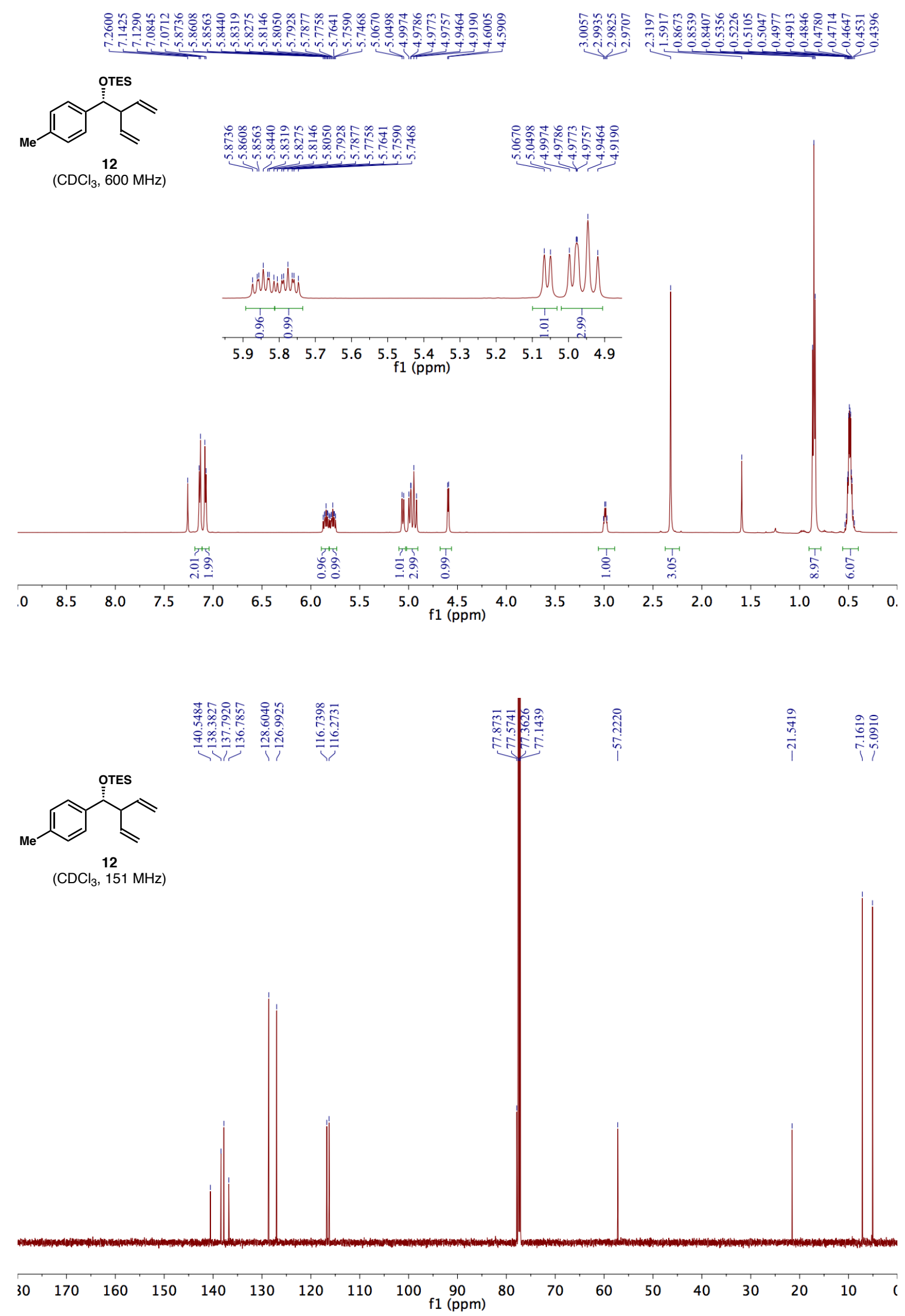

SI-88 

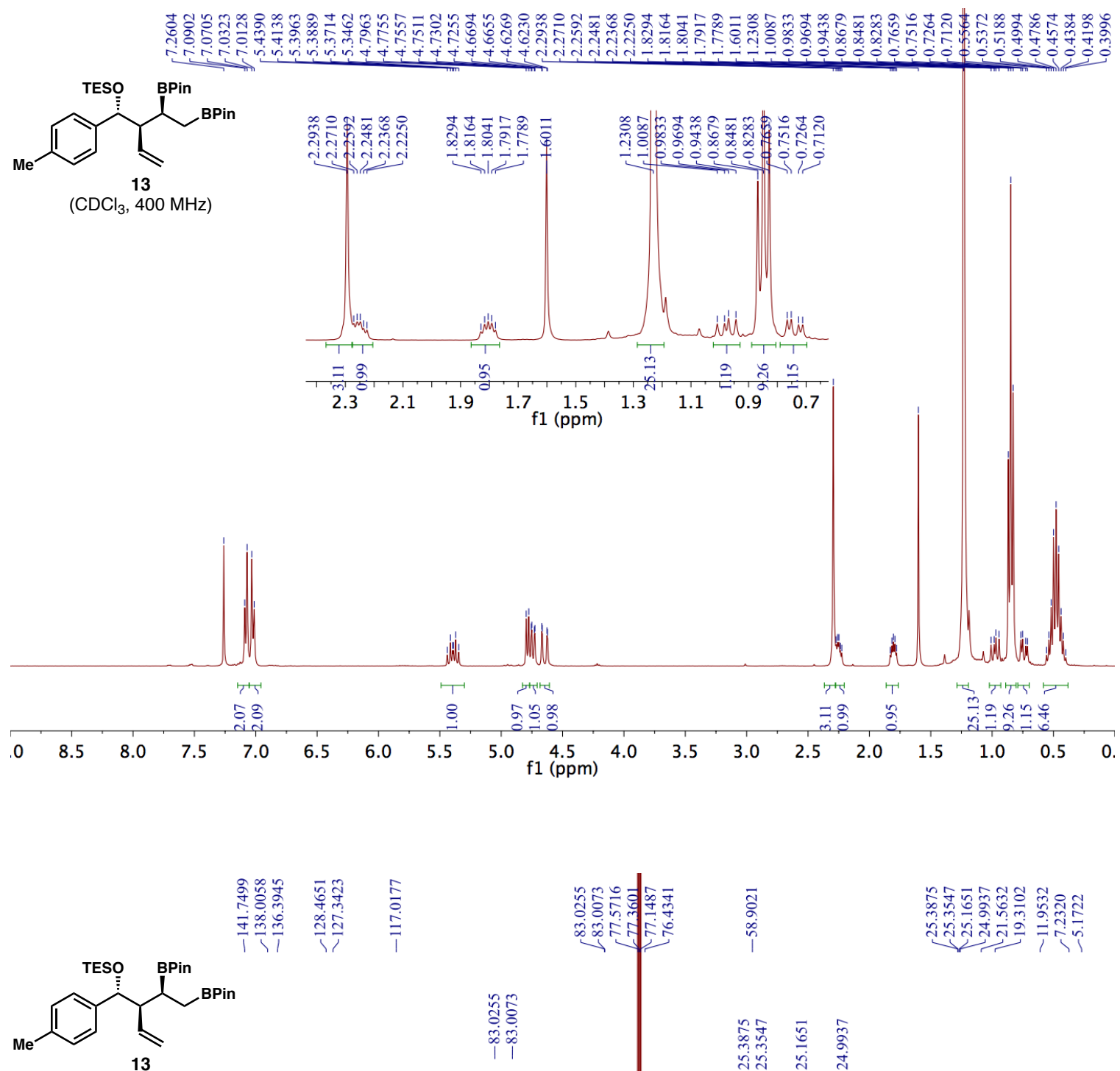

$\left(\mathrm{CDCl}_{3}, 151 \mathrm{MHz}\right)$

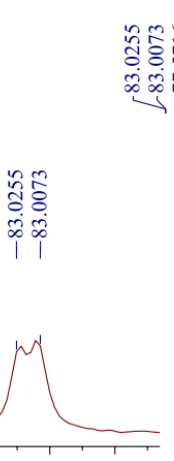

83.0583 .0082 .95 f1 (ppm)

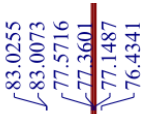

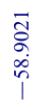

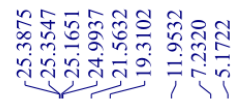

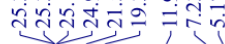

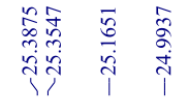

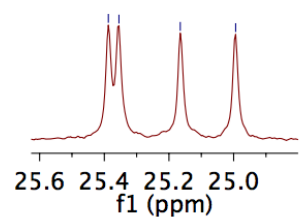

$25.6 \underset{f 1}{25.4} 25.225$
(ppm)

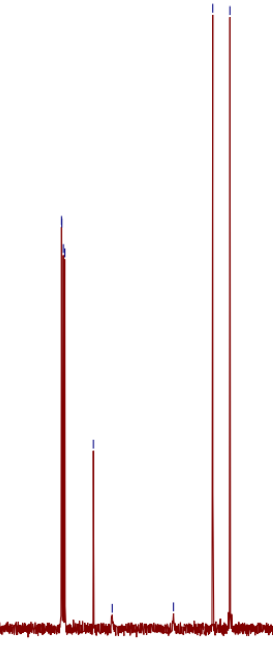

30

$170 \quad 160$

$\begin{array}{llll}50 & 140 & 130 & 120\end{array}$

$110 \quad 100$ 

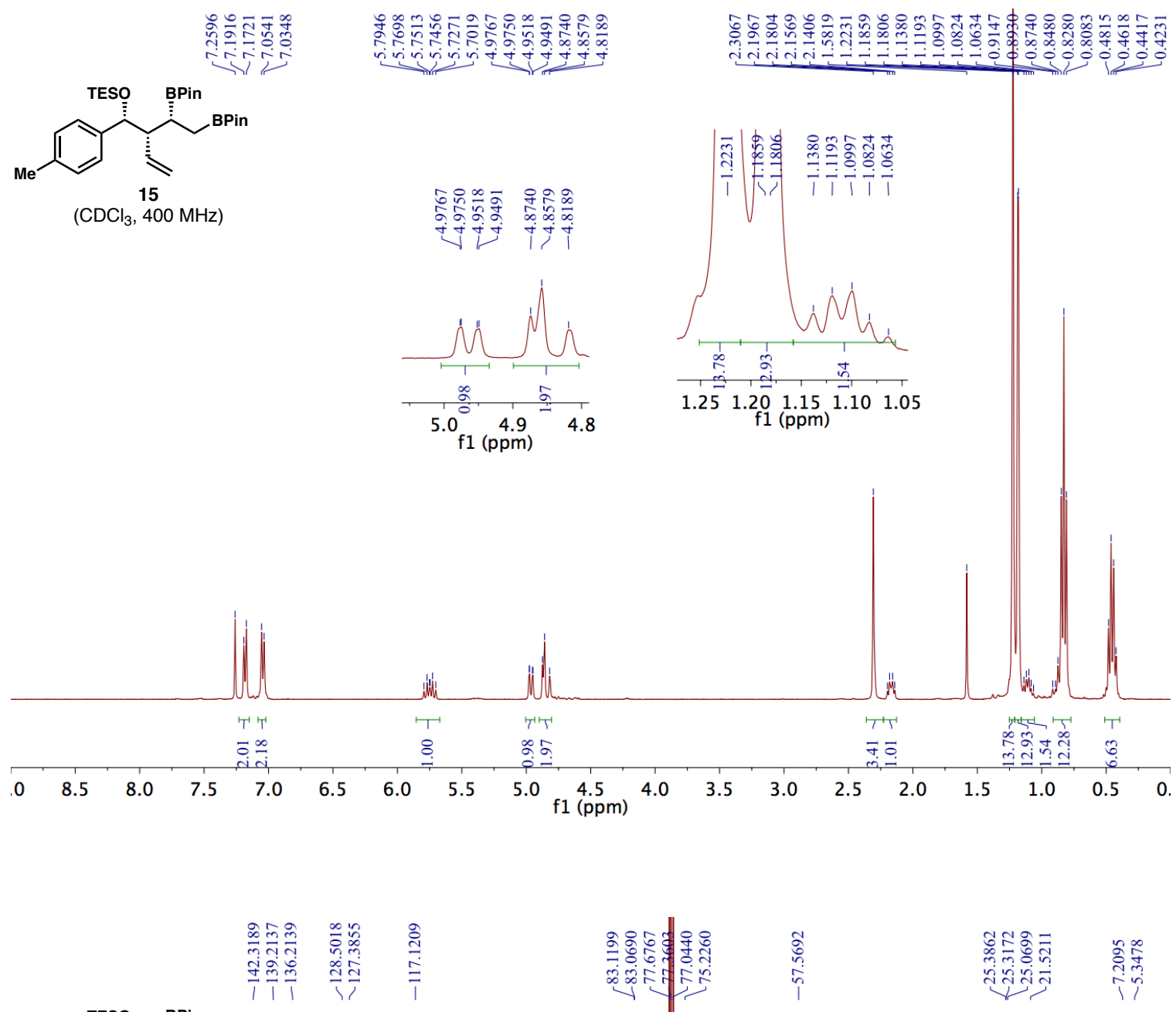

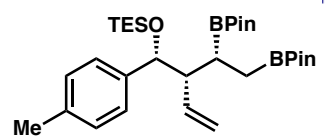

15

$\left(\mathrm{CDCl}_{3}, 101 \mathrm{MHz}\right)$
28
$\vdots 0$
$\vdots 0$
$\infty$
11
11

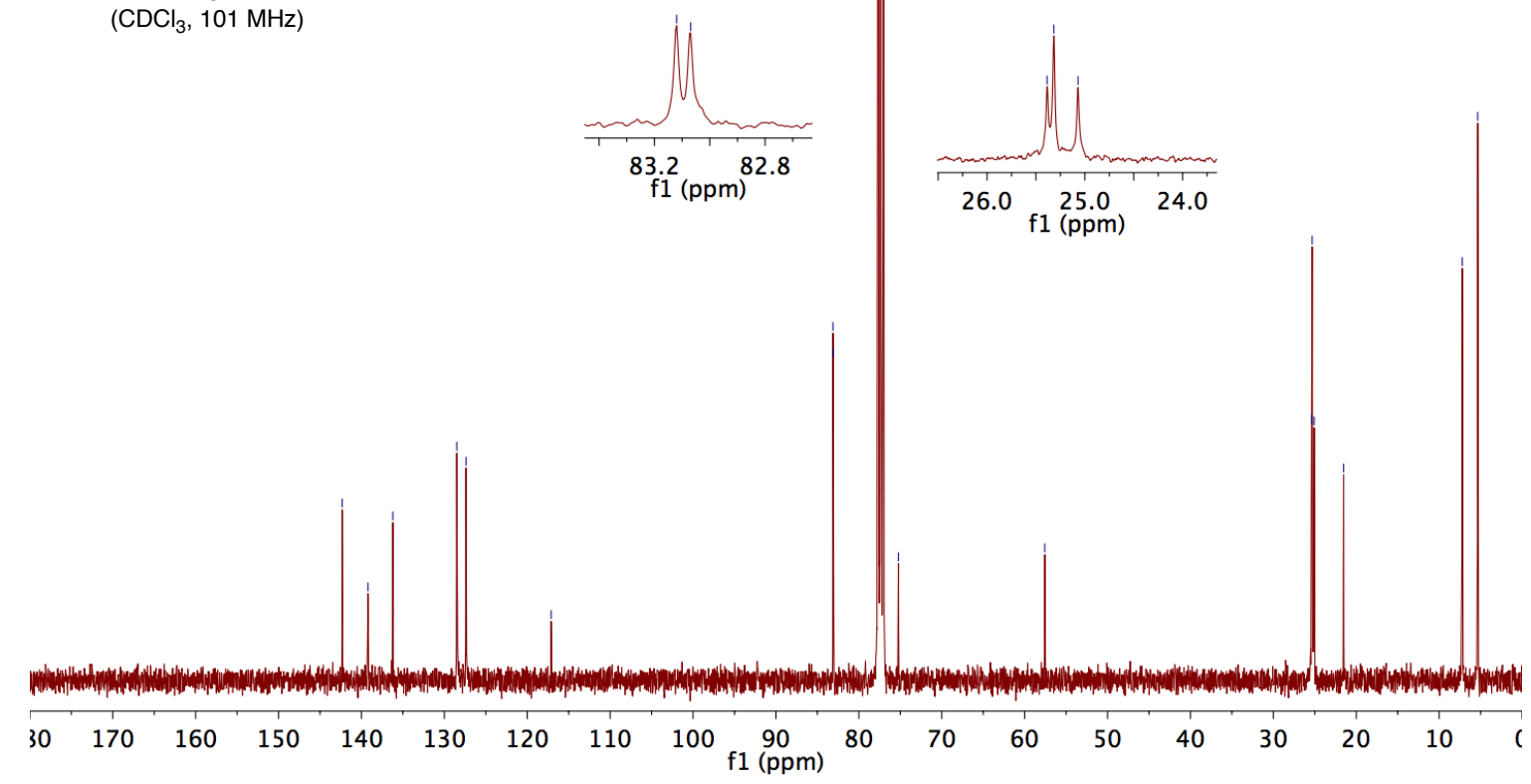

ज证

गैं 